\title{
INTEGRAÇÃO DE PROJETO ARQUITETÔNICO E ESTRUTURAL VIA METODOLOGIA BIM
}

\author{
Elaborado por \\ Carlos Teixeira dos Santos Junior
}

Trabalho de Conclusão de Curso

Trabalho apresentado como requisito parcial à conclusão do curso de Engenharia Civil na Pontifícia Universidade Católica do Rio de Janeiro, Rio de Janeiro, Brasil.

Orientador: Prof $^{\circ} \operatorname{Dr}^{\circ}$ Glauco José de Oliveira Rodrigues.

Rio de Janeiro novembro de 2021 


\title{
INTEGRAÇÃO DE PROJETO ARQUITETÔNICO E ESTRUTURAL VIA METODOLOGIA BIM
}

\author{
BANCA EXAMINADORA
}

Prof. Dr ${ }^{\circ}$ Glauco José de Oliveira Rodrigues

(Orientador)

Eng. Natália Victoria dos Santos

(Coorientadora)

Eng. Rodrigo do Val Andrade

(Avaliador)

Aprovado em de de 2021. 


\section{Agradecimentos}

Primeiramente agradeço a Deus por tudo o que tem acontecido na minha vida, por ter me dado a oportunidade de me formar em uma das mais conceituadas Universidades do Brasil.

Agradeço a minha família por todo o apoio que me deram durante toda a faculdade, sempre me apoiando e me incentivando e me dando forças para não desistir.

Agradeço ao meu orientador D. Sc Glauco José de Oliveira Rodrigues por todo o apoio e ensinamento passado durante a elaboração deste trabalho e também aos demais professores da PUC-RIO pelo conhecimento passado contribuindo para a minha formação.

Agradeço aos amigos Rodrigo Guimarães Martins e Aline Correia Gomes que tem acompanhado toda a minha jornada na construção civil há quase 10 anos, e que com certeza sem o apoio e ajuda deles eu não estaria aqui hoje. 


\section{Resumo}

Este trabalho consta do desenvolvimento de um projeto estrutural (concreto armado) e arquitetônico de uma residência unifamiliar utilizando os softwares Revit e Cypecad para dimensionamento e modelagem de acordo com as normas da ABNT (Associação Brasileira de Normas Técnicas). Nele será empregado a metodologia BIM onde será feito a integração entre os projetos e a correção das interferências. O método empregado visa buscar, agilidade, otimização, segurança e economia na elaboração do projeto e na obra.

Palavras-chave: Cálculo estrutural. Arquitetônico. BIM. Revit. Cypecad 
Abstract

This work consists of the development of a structural (reinforced concrete) and architectural project for a single-family house using Revit and Cypecad software for dimensioning and modeling in accordance with ABNT (Brazilian Association of Technical Standards) standards. In it, the BIM methodology will be used, where the integration between the projects and the correction of interferences will be carried out. The method used aims to seek, agility, optimization, safety and economy in the preparation of the project and in the work.

Keywords: Structural calculation. Architectural. BIM. Revit. Cypecad 


\section{SUMÁRIO}

1 INTRODUÇÃO......................................................................................... 1

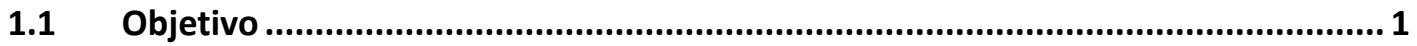

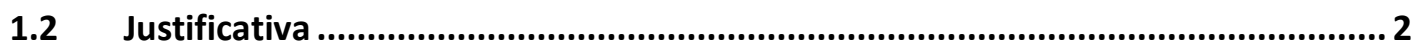

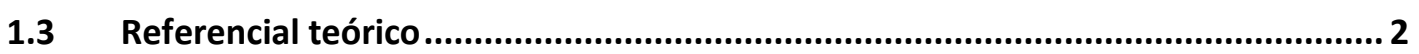

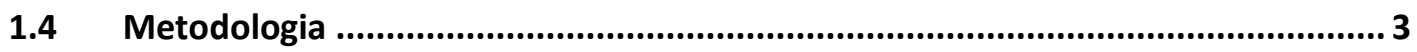

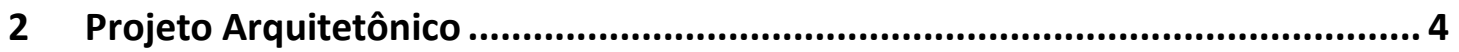

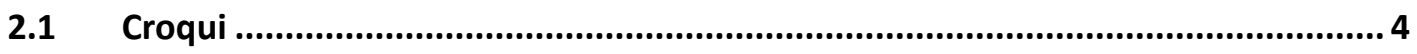

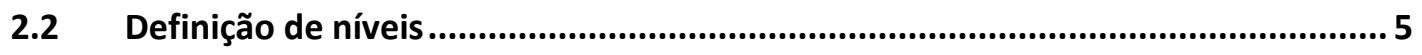

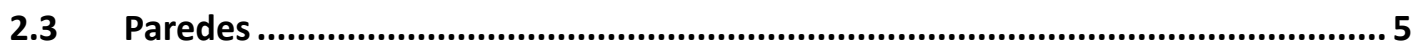

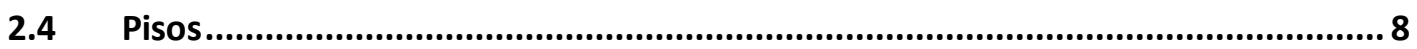

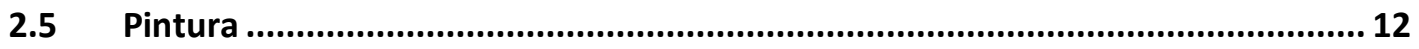

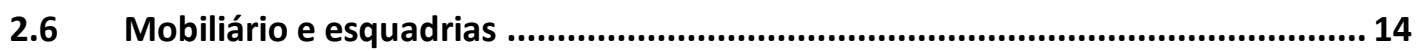

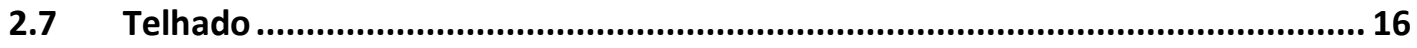

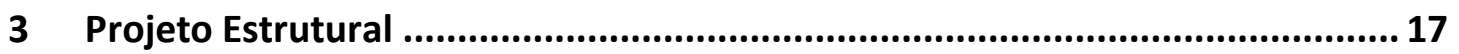

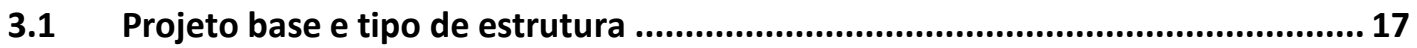

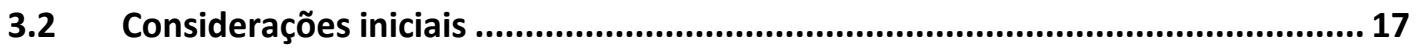

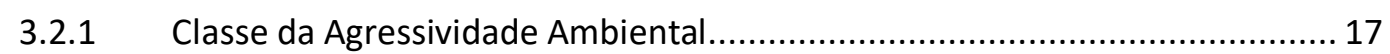

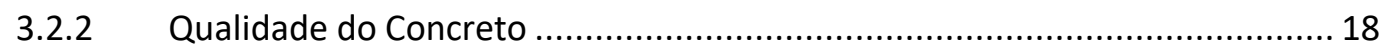

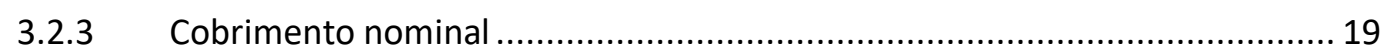

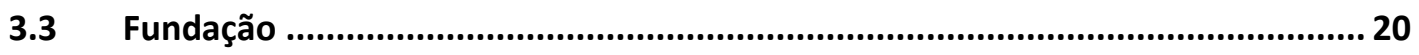

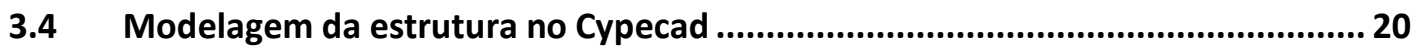

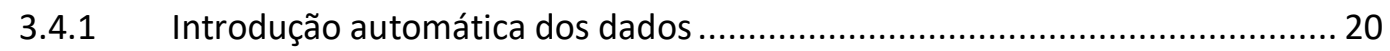

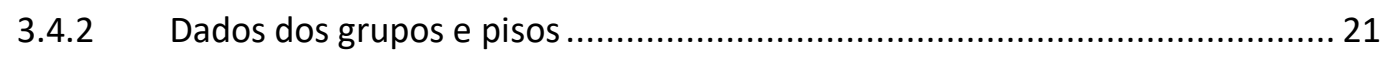

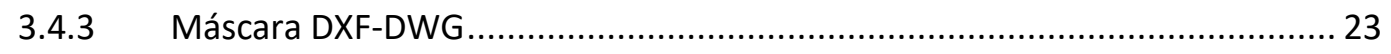

3.4.4 Dados Gerais (Materiais utilizados, Fissuras, cobrimento e tensão admissível do solo) 23

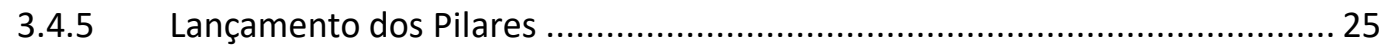

3.4.6 Lançamento e Pré-dimensionamento das vigas .......................................... 27

3.4.7 Lançamento e Pré-dimensionamento das lajes............................................ 30

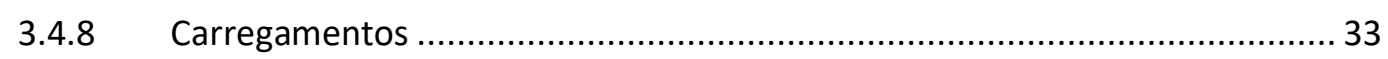

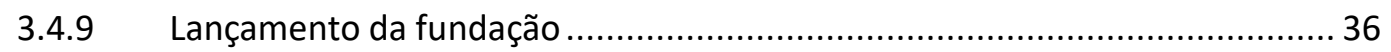

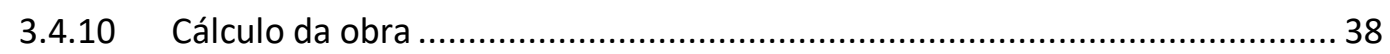

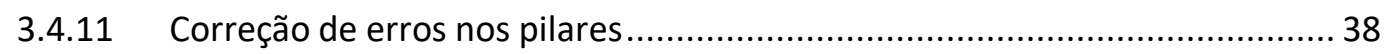

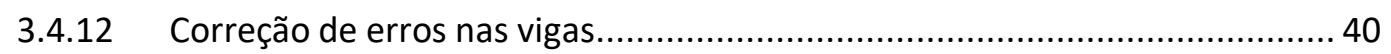

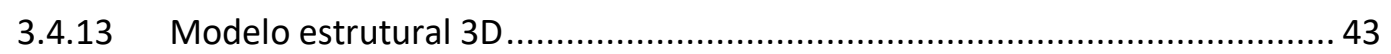

3.4.14 Otimização da armadura das vigas ........................................................... 43

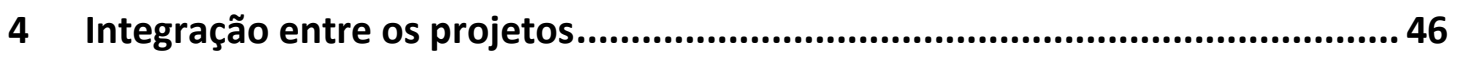

4.1 Exportação do ifc do Cypecad para o Revit .......................................................... 46 


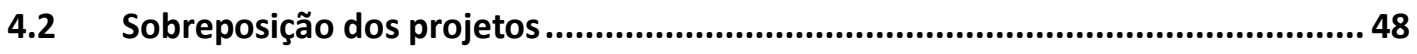

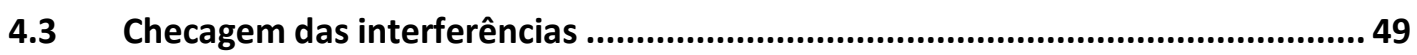

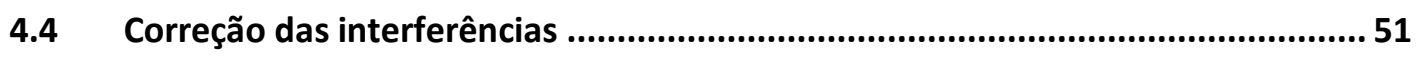

4.5 Integração entre os projetos finalizados .......................................................... 52

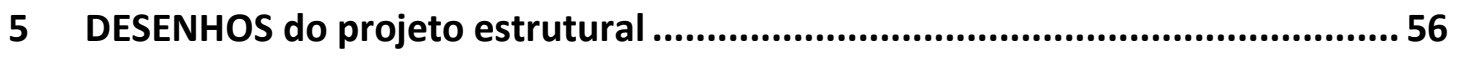

6 MEMÓRIA DE CÁLCULO RESUMIDA ........................................................... 72

todos os dados deste capítulo foram importados do Cypecad .............................. 72

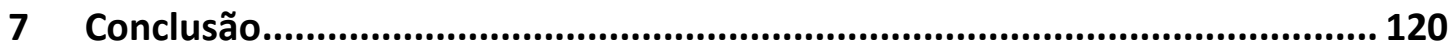

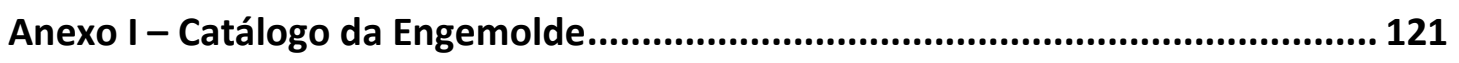

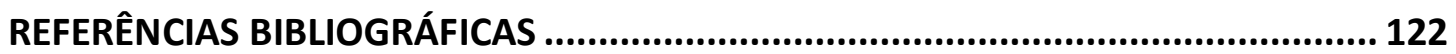




\section{ÍNDICE DE FIGURAS}

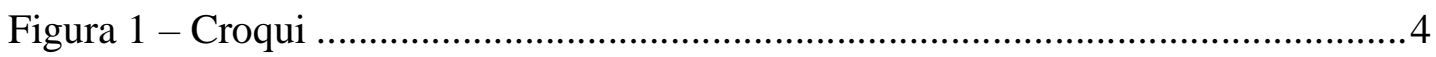

Figura 2 - Níveis da edificação.................................................................................

Figura 3 - Materiais das paredes..........................................................................

Figura 4 - Paredes do térreo...................................................................................

Figura 5 - Paredes da laje de cobertura.......................................................................

Figura 6 - Materiais do piso do térreo...................................................................

Figura 7 - Materias da laje de cobertura ................................................................9

Figura 8 - Piso do térreo …………………………………………………........ 10

Figura 9 - Piso da laje de cobertura .........................................................................11

Figura 10 - Paredes do térreo sem aplicação de pintura...............................................12

Figura 11 - Paredes da térreo após a aplicação da pintura.............................................13

Figura 12 - Paredes externas de toda a edificação após a pintura...................................14

Figura 13 - Inserção do mobiliário e esquadrias -2D...............................................15

Figura 14 - Inserção do mobiliário e esquadrias - 3D...................................................16

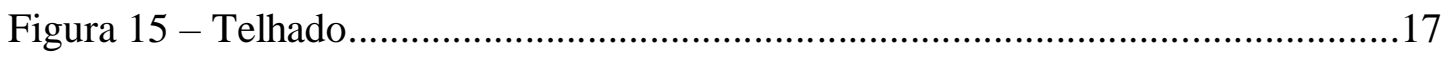

Figura 16 - Classe de agressividade ambiental.......................................................18

Figura 17 - Relação entre a classe de agressividade e qualidade do concreto..............19

Figura 18 - Relação entre a classe de agressividade e o cobrimento nominal..............20

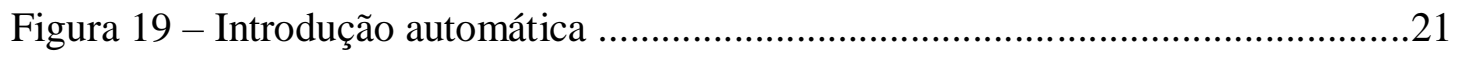

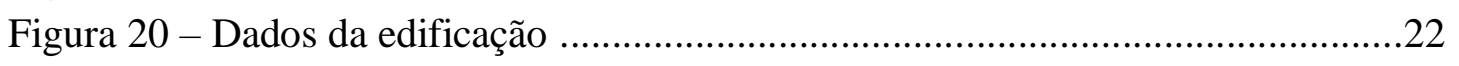

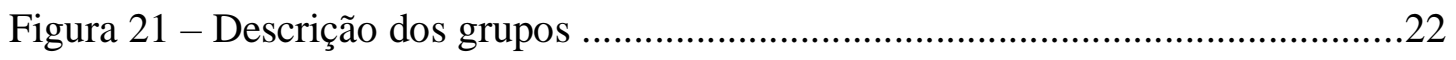

Figura 22 - Especificações de concreto e aço ………………………………….......24

Figura 23 - Fissuras e cobrimento ……………………………………………......

Figura 24 - Tensões admissíveis no solo de apoio ...................................................25

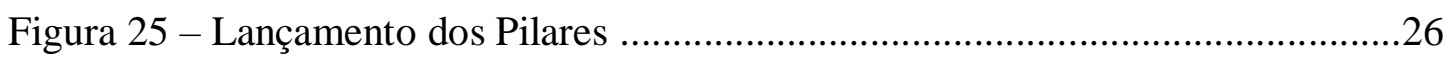

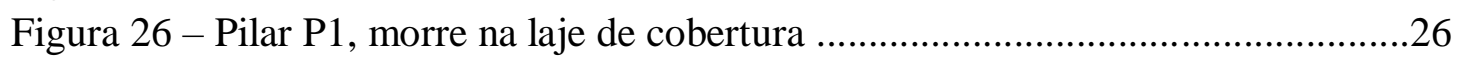

Figura 27 - Pilar P4, morre na laje do térreo (cintamento) .........................................27

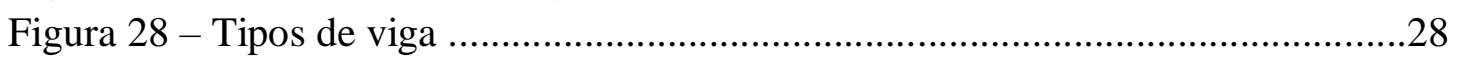

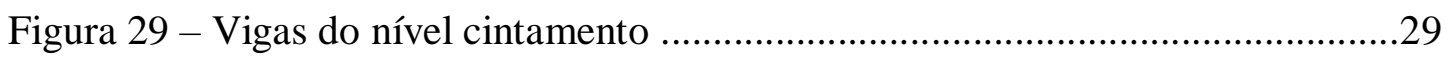

Figura 30 - Vigas do nível cobertura ..........................................................................

Figura 31 - Vãos máximos para lajes treliçadas............................................................31

Figura 32 - Criação do bloco para lajes pré-moldada ……………………………......32

Figura 33 - Lançamento da pré-laje no nível de cobertura ...........................................33

Figura 34 - Cargas de alvenaria sobre as vigas do cintamento …………………….........

Figura 35 - Cargas de alvenaria e caixa d'água no nível da cobertura .........................36

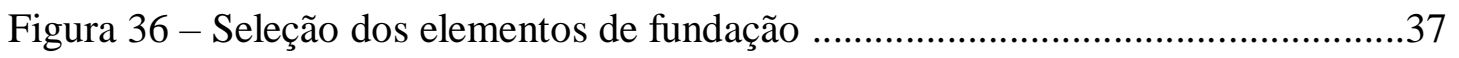

Figura 37 - Lançamento da fundação ……………………………………………......

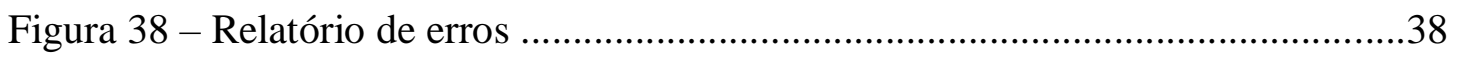


Figura 39 - Erro nos pilares .39

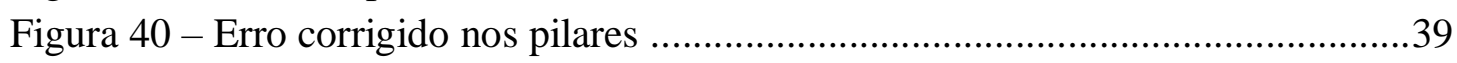

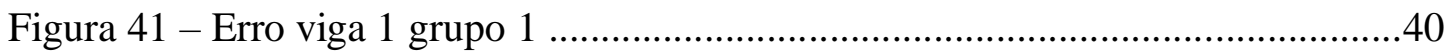

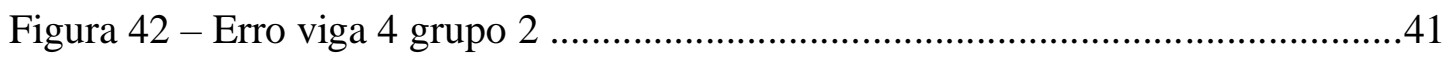

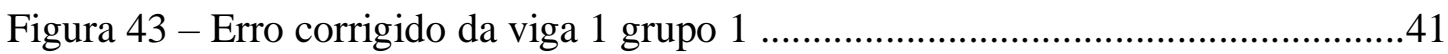

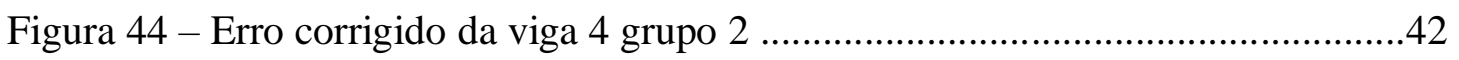

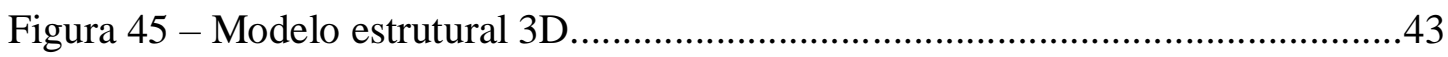

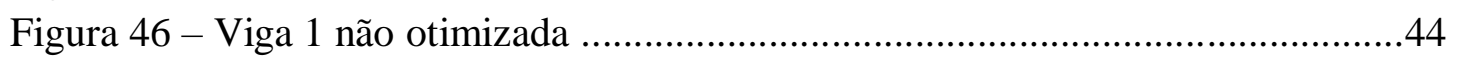

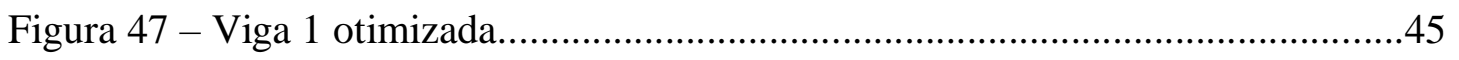

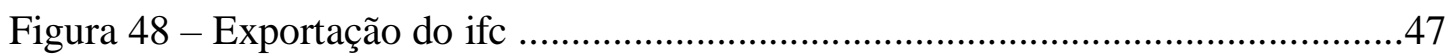

Figura 49 - Modelo estrutural exportado para o Revit, com ifc ...............................47

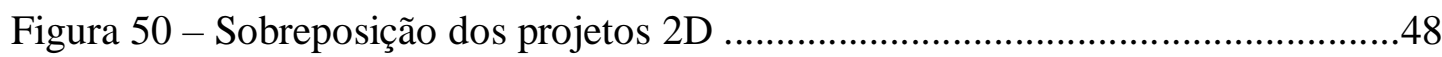

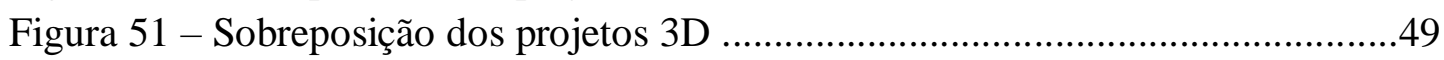

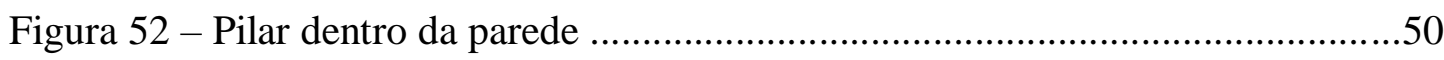

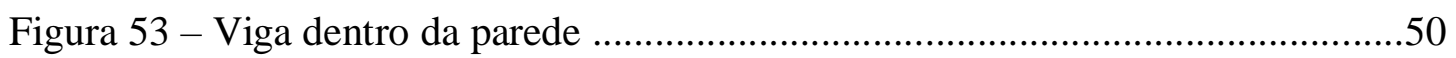

Figura 54 - Interferência pilar/parede corrigida .......................................................51

Figura 55 - Interferência viga/parede corrigida ...................................................52

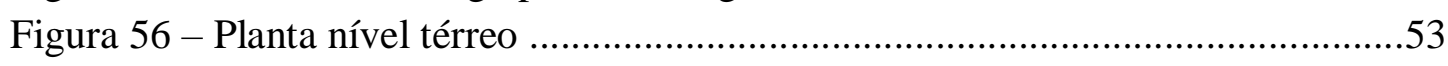

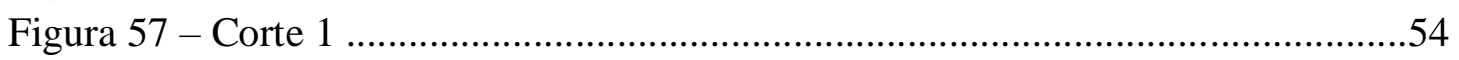

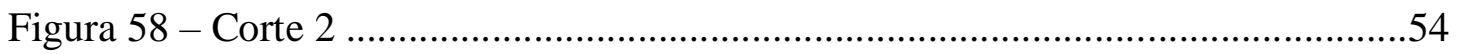

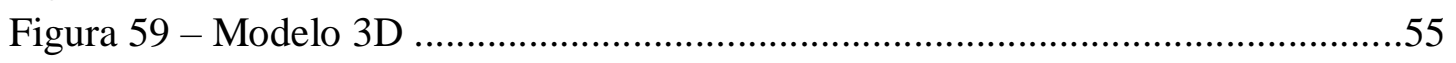

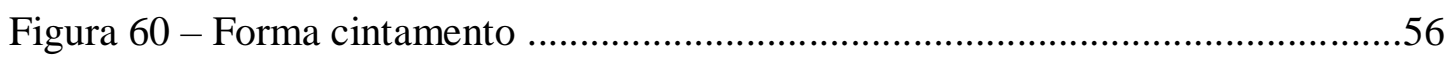

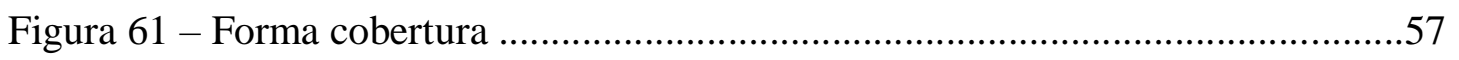

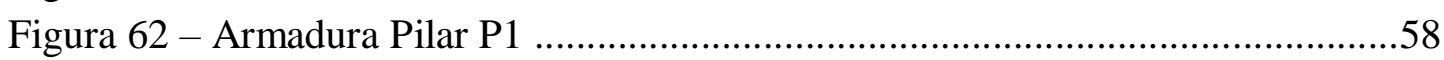

Figura 63 - Armadura Pilares P2 e P3 ................................................................59

Figura 64 - Armadura Pilares P4, P9 e P13 ........................................................60

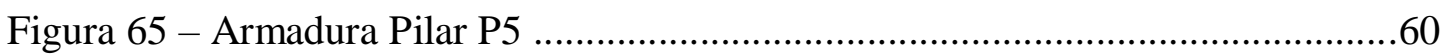

Figura 66 - Armadura Pilares P6, P7, P8, P10, P11, P12 e P14 ................................61

Figura 67 - Armadura Pilar 15 ..............................................................................62

Figura 68 - Armadura Pilar 16 ..............................................................................63

Figura 69 - Armadura vigas V1 e V2 - cintamento ..................................................63

Figura 70 - Armadura vigas V3, V4 e V5 - cintamento .............................................64

Figura 71 - Armadura vigas V6 e V7 - cintamento ......................................................64

Figura 72 - Armadura vigas V8 e V9 - cintamento ................................................65

Figura 73 - Armadura vigas V10, V11 e V12 - cintamento ......................................65

Figura 74 - Armadura vigas V13, V14, V15 e V16 - cintamento ...............................66

Figura 75 - Armadura vigas V17 - cintamento .......................................................66 
Figura 76 - Armadura vigas V1 e V2 - cobertura .67

Figura 77 - Armadura vigas V3 e V8 - cobertura …...........................................67

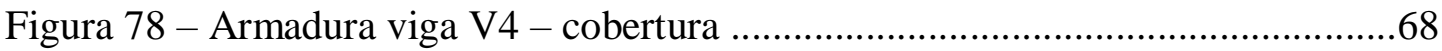

Figura 79 - Armadura viga V5 - cobertura .........................................................68

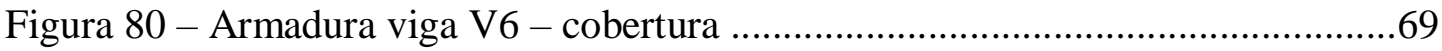

Figura 81 - Armadura viga V7 - cobertura .......................................................69

Figura 82 - Armadura viga V5 - cobertura.......................................................... 70

Figura 83 - Armadura das sapatas referentes aos pilares P1, P2, P6, P7, P8, P10, P12,

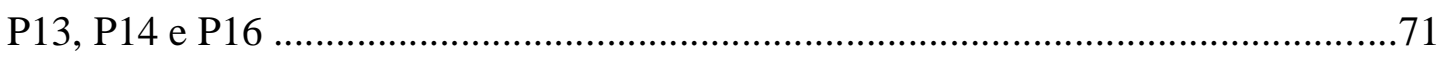

Figura 84 - Armadura das sapatas referentes aos pilares P3, P4 e P9 ........................71

Figura 85 - Armadura das sapatas referentes aos pilares P5, P11 e P15....................72 


\section{INTRODUÇÃO}

Com o passar dos anos a engenharia civil vem evoluindo cada vez mais no setor de softwares voltados para a elaboração de projetos, permitindo o desenvolvimento desses projetos seja para pequeno ou grande porte. Esse desenvolvimento traz uma redução no tempo e no custo desta atividade quando comparado com os métodos tradicionalmente manuais.

Hoje em dia muitas empresas estão se adaptando a nova tecnologia de trabalho chamada BIM (Building Information Modeling) ou Modelagem de Informação na Construção, esta tecnologia permite fazer a integração de projetos através da sua sobreposição e migração entre diversas plataformas.

Com base nisso este trabalho visa desenvolver a integração de um projeto arquitetônico e estrutural (projeto em concreto armado) de uma residência unifamiliar de um pavimento, situada no bairro de Campo Grande - Rio de Janeiro, através dos softwares Revit e Cypecad. Com esta integração será possível fazer as verificações das interferências entre o projeto estrutural (desenvolvido no Cypecad) e o projeto Arquitetônico (desenvolvido no Revit).

\subsection{Objetivo}

Este trabalho tem como objetivo o desenvolvimento do projeto arquitetônico e estrutural (concreto armado), com os softwares Revit e Cypecad de uma residência unifamiliar de um pavimento, mostrando a integração entre eles através da tecnologia BIM, de modo a fazer a compatibilização entre as disciplinas após a correção das interferências. 


\subsection{Justificativa}

Com o passar dos anos a tecnologia está cada vez mais presente na área da construção civil. Hoje em dia, com a criação de vários softwares, muitos trabalhos tiveram o seu tempo de execução reduzido, graças a eles, mantendo a mesma qualidade.

Para isso, é preciso que o profissional esteja atento as mudanças do mercado e que também tenha domínio tanto teórico quanto da utilização destes softwares.

Contudo, este trabalho irá apresentar a junção do conhecimento teórico com o conhecimento tecnológico no uso de 2 softwares muito usados na atualidade, Revit (projeto arquitetônico) e Cypecad (projeto estrutural em concreto armado), mostrando como a junção deles é capaz de trazer mais economia e segurança.

Neste trabalho será mostrado uma das formas de integração entre projetos de diferentes disciplinas, no caso arquitetura e estrutural, onde através da sobreposição dos projetos será possível verificar as interferências entre os mesmos utilizando o conceito BIM.

\subsection{Referencial teórico}

Este trabalho terá como referência os conceito de BIM e IFC aplicado nas disciplinas de arquitetura e estruturas, apresentando 2 softwares compatíveis para esta metodologia: Autodesk REVIT (2021) e CYPECAD (2017).

O conceito de BIM (Building Information Model) que em português pode ser traduzido para "Modelo de Informação da Construção" não se trata de um software específico, e sim de um conceito de virtualização, modelagem e gerenciamento das atividades inerentes ao projeto/construção de obras de engenharia. O projeto, neste novo conceito, torna-se muito mais próximo da obra real (virtualização dos elementos), facilitando a observação de possíveis inconformidades (erros de projeto, sobreposições, etc.). A representação planificada deixa de ser o meio para o desenvolvimento do projeto e torna-se um dos fins disponíveis de representação. (SAEPRO, 2015). 
O IFC (Industry Foundation Classes) é um formato de arquivo que contém dados (Informação), cuja finalidade permite o intercâmbio de informações para um modelo informativo sem perda ou distorção de dados. O IFC não descreve apenas a geometria, mas muito mais do que isso. Aqui estamos falando de semântica, em um processo BIM, não estamos apenas trocando geometria, mas também dados. Neste momento, as informações anexadas a um objeto são descritas em Conjuntos de Propriedades. Um Conjunto de Propriedades é o espaço reservado onde as propriedades definidas podem ser anexadas a qualquer entidade dentro do modelo IFC. (MAKEBIM, 2017).

O Revit é um software de BIM que traz todas as disciplinas de arquitetura, engenharia e construção para um ambiente de modelagem unificado, gerando projetos mais eficientes e econômicos (AUTODESK, 2015).

O CYPECAD é um software para projeto de cálculo estrutural em concreto armado, pré-moldado, protendido e misto de concreto e aço que engloba as etapas de lançamento do projeto, análise e cálculo da estrutura, dimensionamento e detalhamento final dos elementos. Os recursos de detalhamento e dimensionamento estão de acordo com as normas brasileiras de concreto armado (NBR 6118:2014), fundações (NBR 6122), carregamentos (NBR 6120), barras (NBR 7480), ventos (NBR 6123), ações e combinações (NBR 8681). (MULTIPLUS, 2015).

Para este trabalho foi utilizado o Revit para a elaboração do projeto arquitetônico e o Cypecad para dimensionamento e modelagem do projeto estrutural em concreto armado. Após a elaboração de ambos os projetos é feito a integração entre eles fazendo a exportação do IFC do Cypecad para o Revit, com isso é possível fazer a verificação e correção das interferências.

\subsection{Metodologia}

Este trabalho será dividido em duas etapas. Na primeira etapa será feito a modelagem da arquitetura no Revit, na segunda etapa será feito a modelagem e o dimensionamento dos elementos estruturais (projeto estrutural) no CYPECAD. Após o dimensionamento do projeto estrutural será feito a exportação do IFC do CYPECAD para o Revit. Com os dois arquivos no modelo rvt. será feito a sobreposição entre eles, com este procedimento será possível observar os pontos de interferências, entre ambos os projetos, que deverão ser corrigidos (integração entre os projetos). 


\section{Pontifícia Universidade Catálica $_{\text {a }}$}

\section{PROJETO ARQUITETÔNICO}

\subsection{Croqui}

Todo o projeto arquitetônico foi desenvolvido com o software Revit, porém foi montado um croqui no software AutoCAD (conforme pode ser visto na figura 1) que serviu como base para a elaboração do projeto arquitetônico.

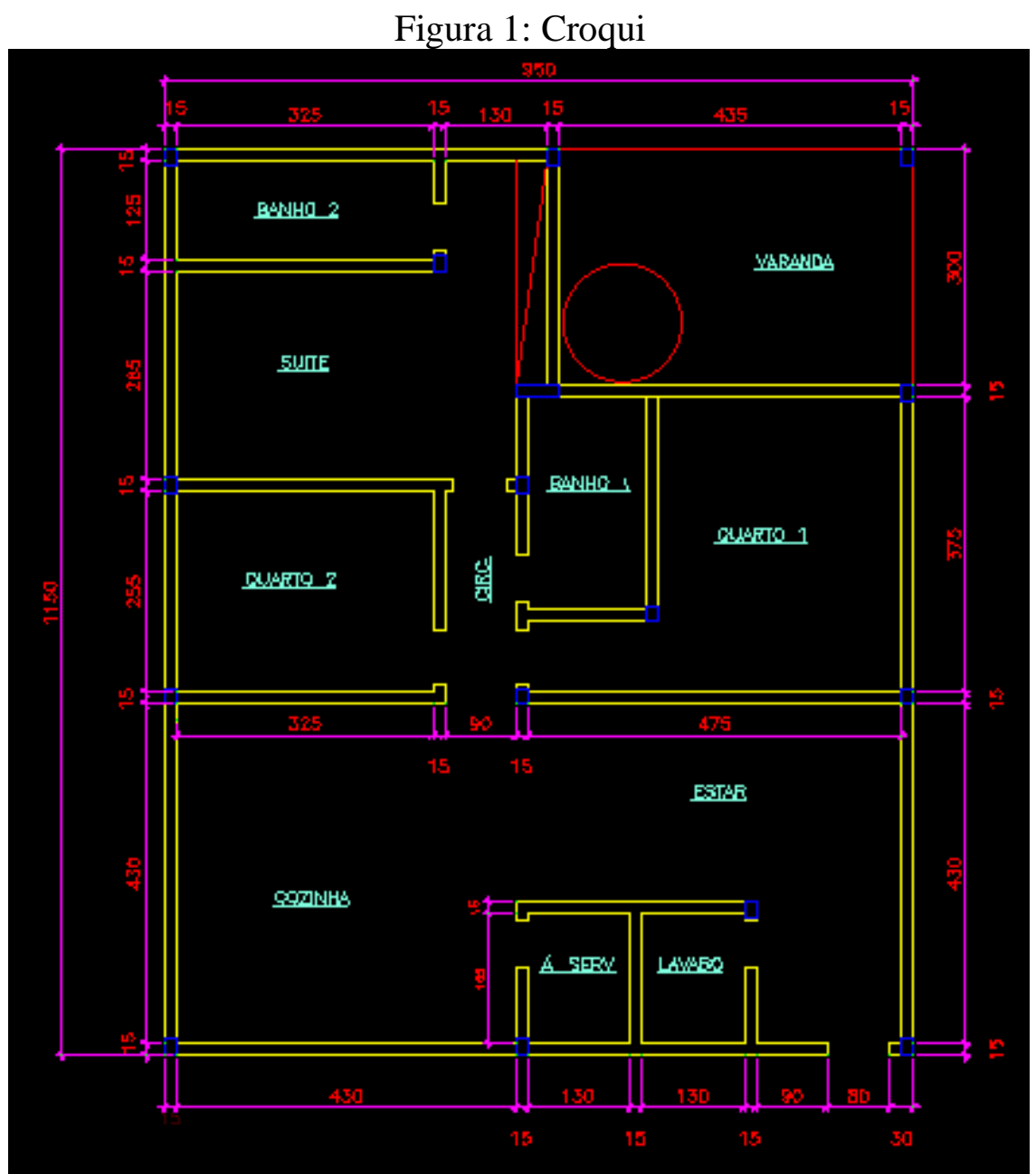

Fonte: Software AutoCAD (2019), 2021 


\subsection{Definição de níveis}

Os níveis (figura 2) são responsáveis pela definição da elevação de um plano horizontal em relação ao outro. A edificação é composta de 1 pavimento, que possui um pé direito de 3,15 m e o topo do telhado (cumeeira) está a 2,00 metros acima da laje da cobertura.

Figura 2: Níveis da edificação

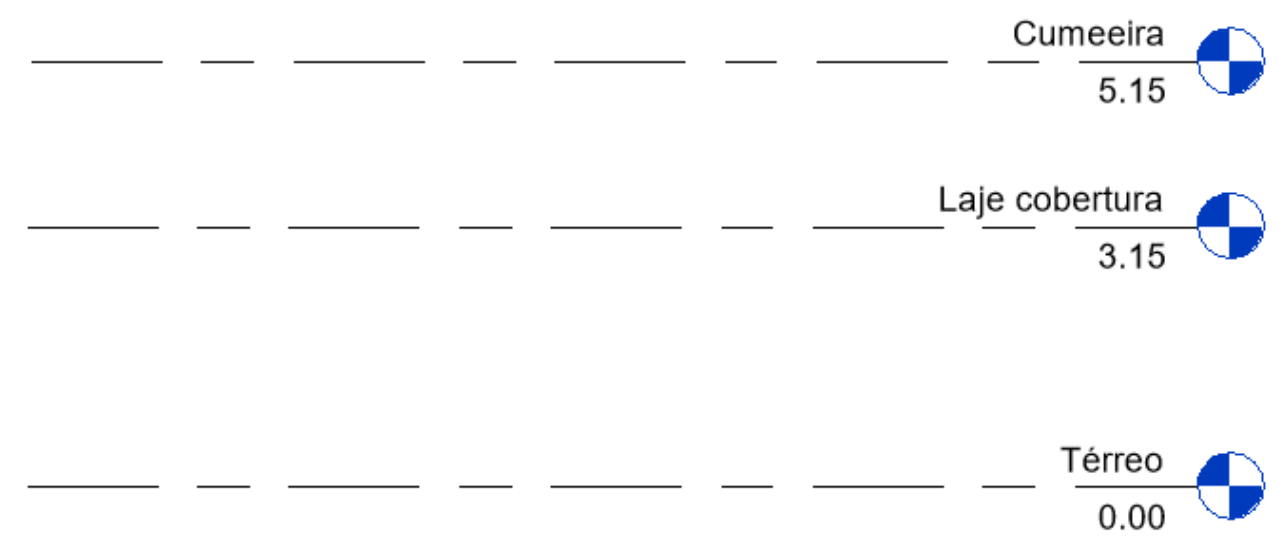

Fonte: Software Revit (2021), 2021

\subsection{Paredes}

\subsubsection{Material}

No Revit é possível fazer a escolha dos materiais que irão compor as paredes (figura 3). Nesta edificação, todas as paredes serão de alvenaria (tijolo de $12 \mathrm{~cm}$ de espessura), com uma camada de 1,5 $\mathrm{cm}$ de reboco em ambos os lados, totalizando uma parede com $15 \mathrm{~cm}$ de espessura. 


\section{Pontifícia Universidade Catálica

Figura 3: Materiais das paredes

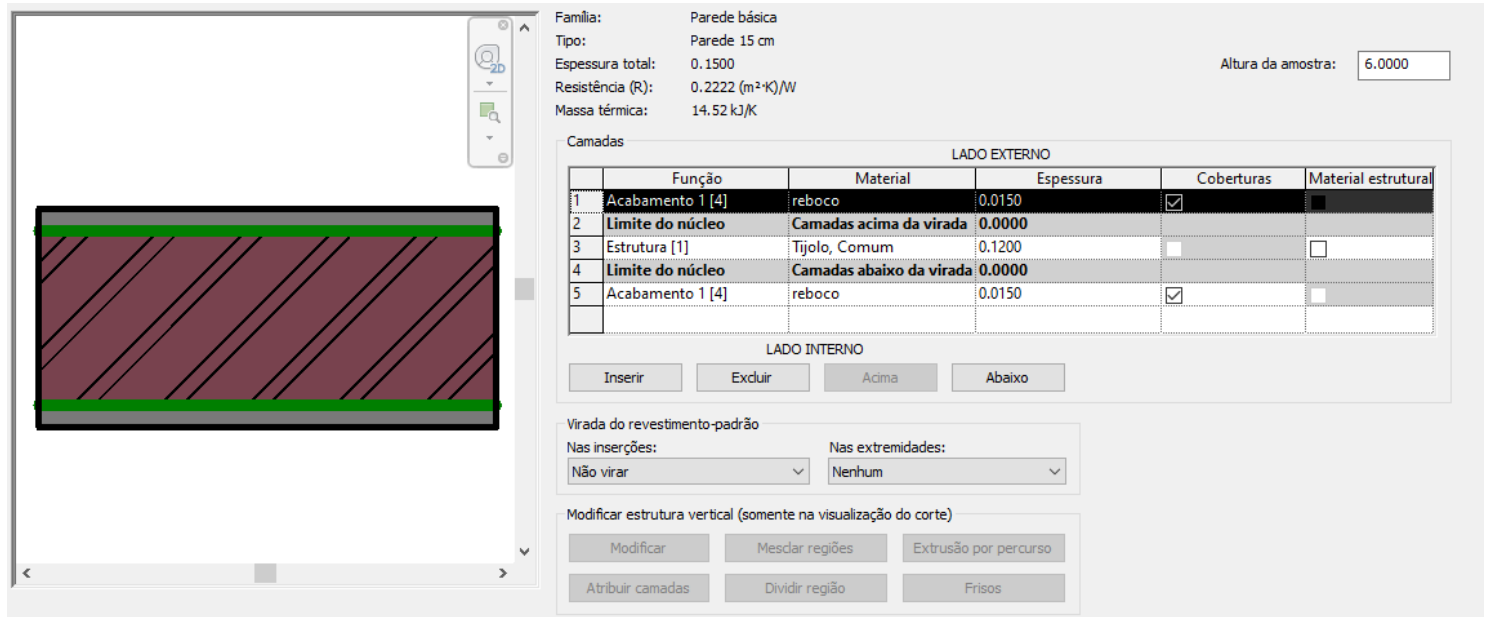

Fonte: Software Revit (2021), 2021

\subsubsection{Traçado das paredes do térreo}

Com base no croqui elaborado no software AutoCAD, foi feito o traçado das paredes internas e externas da edificação do nível térreo (figura 4), que irá fazer parte da planta baixa do térreo. 


\section{Pontifícia Universidade Catálica

Figura 4: Paredes do térreo

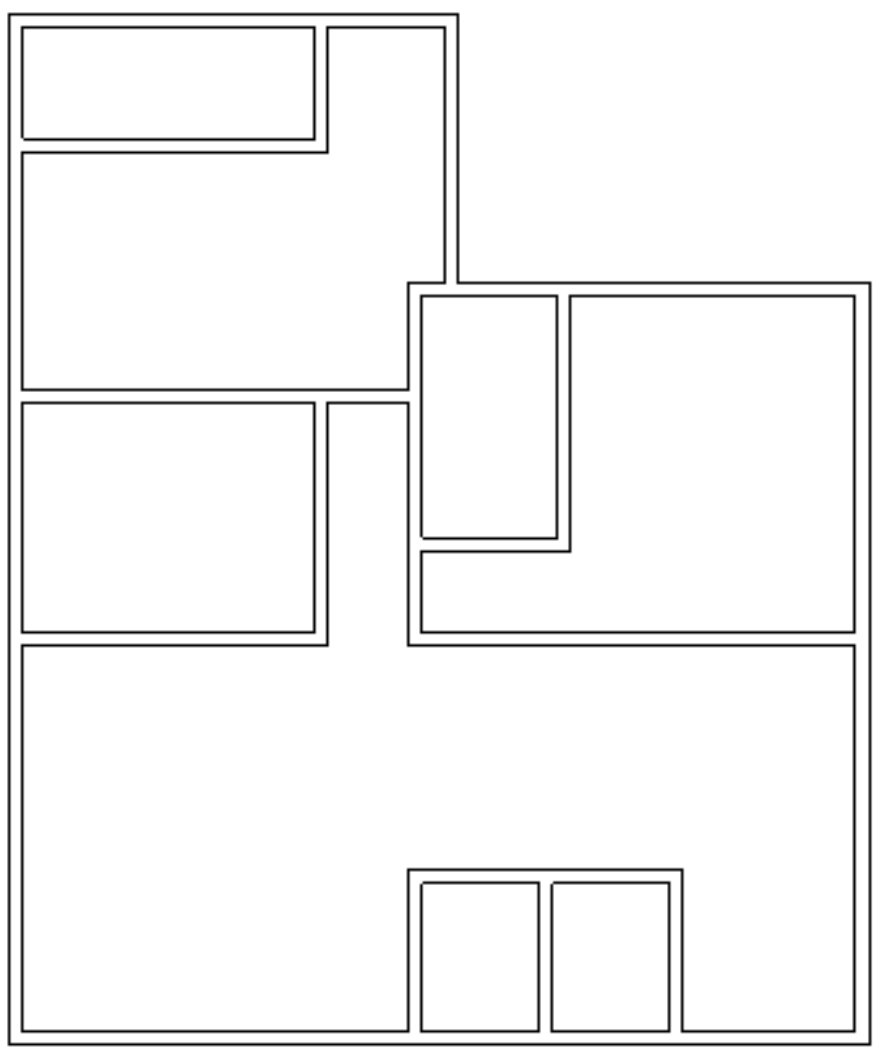

Fonte: Software Revit (2021), 2021

\subsubsection{Traçado das paredes da laje de cobertura}

Para a sustentação do telhado, foi implantado paredes no nível da laje de cobertura (conforme pode ser visto na figura 5). 
Figura 5: Paredes da laje de cobertura

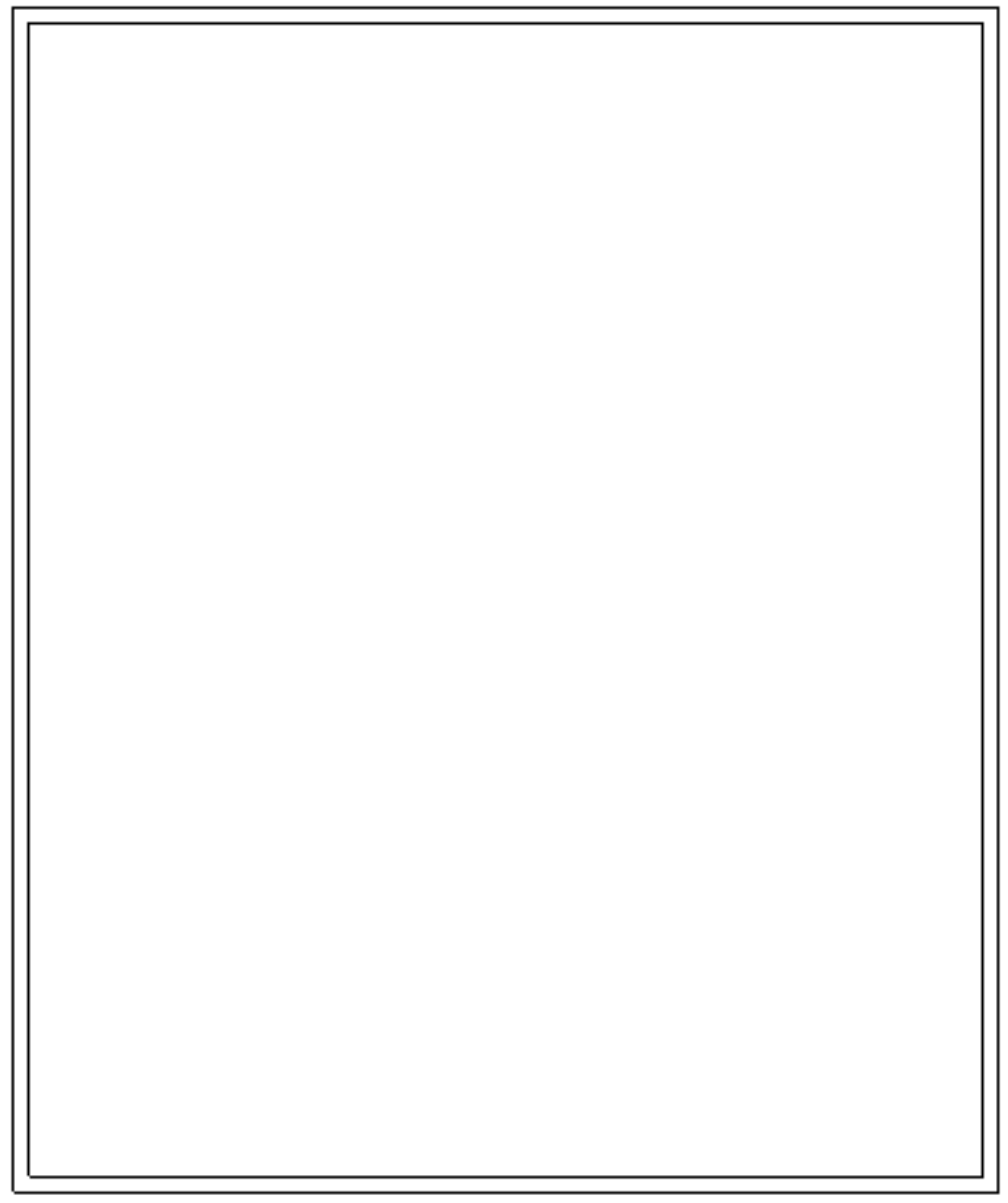

Fonte: Software Revit (2021), 2021

\subsection{Pisos}

\subsubsection{Material}

Assim como nas paredes, nos pisos também é possível fazer a escolha do material que irá compor os pisos. O piso do térreo, figura 6 , será composto por uma camada de concreto de $9,5 \mathrm{~cm}$ de espessura e um revestimento cerâmico de $0,5 \mathrm{~cm}$ de espessura, totalizando $10 \mathrm{~cm}$ de espessura no piso do térreo. A laje de cobertura, figura 7 , será composta apenas por uma camada de concreto de $12 \mathrm{~cm}$ 


\section{Pontifícia Universidade Catálica

Figura 6: Materiais do piso do térreo

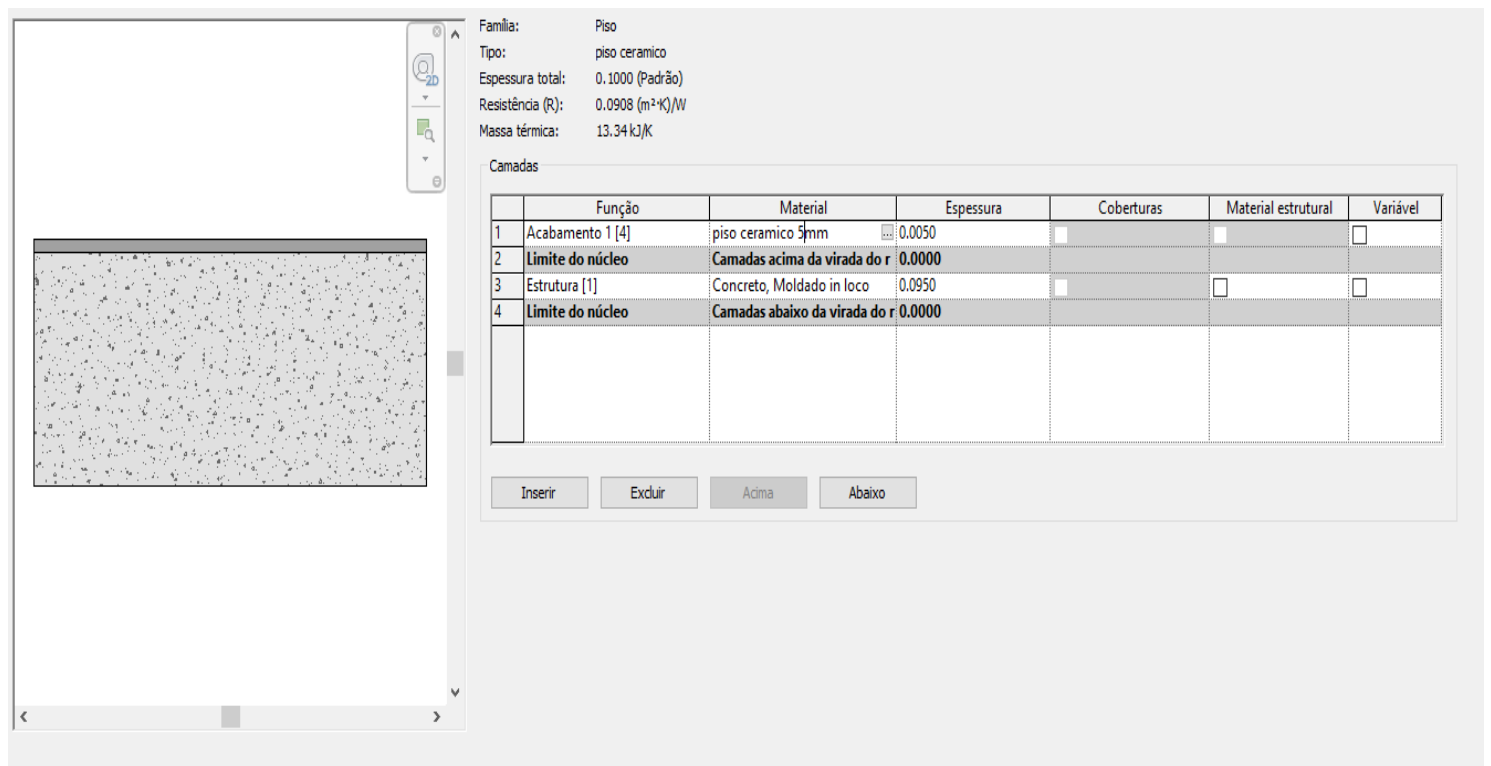

Fonte: Software Revit (2021), 2021

Figura 7: Materiais da laje de cobertura

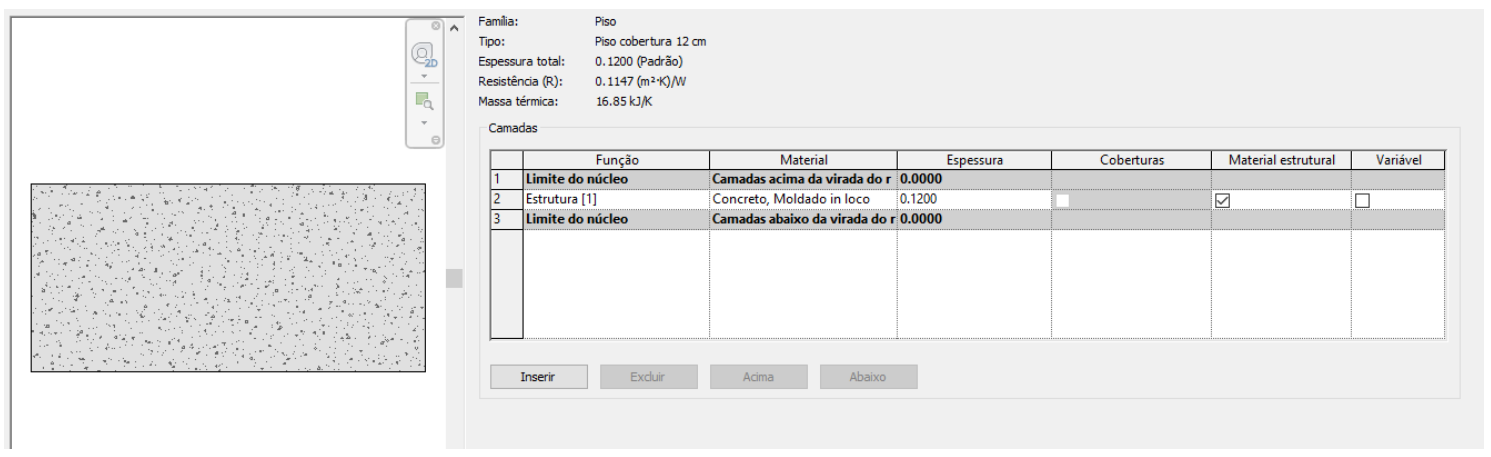

Fonte: Software Revit (2021), 2021

\subsubsection{Traçado do piso do térreo e da laje de cobertura}

Com base no croqui elaborado no software AutoCAD, foi feito o traçado do piso do térreo (figura 8) e da laje de cobertura (figura 9). 


\section{Pontifícia Universidade Católlca $_{\text {a }}$ DO RIO DE JANEIRO}

Figura 8: Piso do térreo

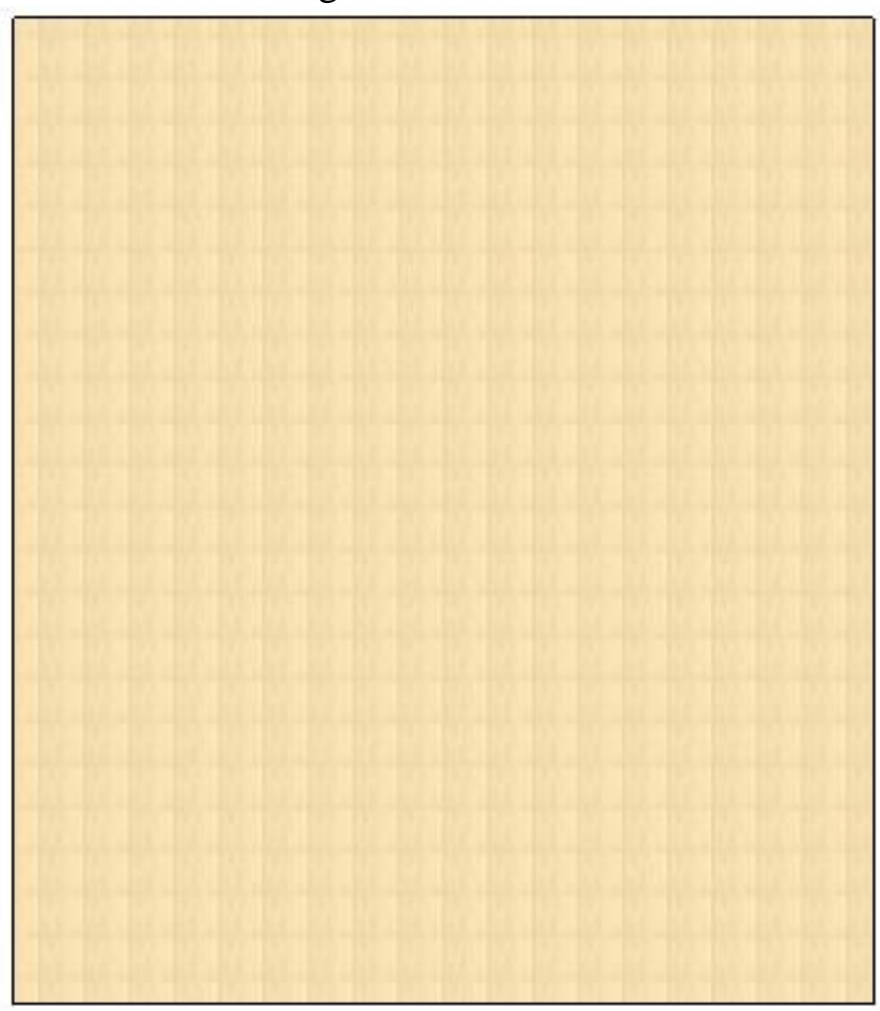

Fonte: Software Revit (2021), 2021 


\section{Pontifícia Universidade Católlica DO RIO DE JANEIRO}

Figura 9: Piso da laje de cobertura

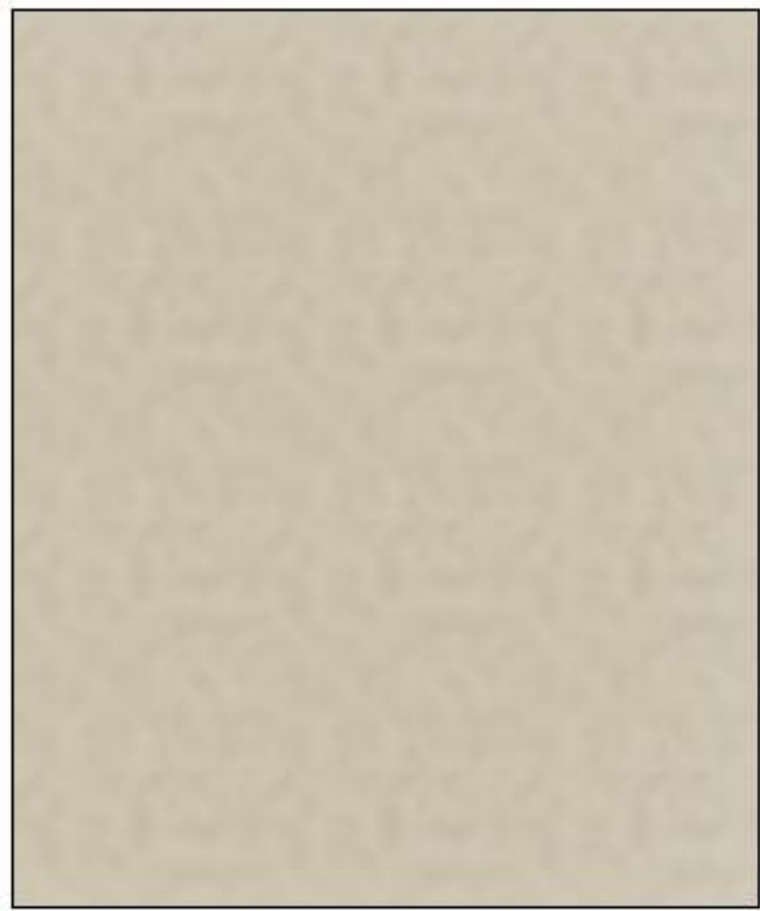

Fonte: Software Revit (2021), 2021 


\section{Pontifícia Universidade Catállica $_{\text {a }}$

\subsection{Pintura}

Após o traçado das paredes é feito a pintura das mesmas (figuras 10, 11 e 12). Cores meramente ilustrativas, ainda não foi definido a real cor das paredes.

Figura 10: Paredes do térreo sem aplicação de pintura

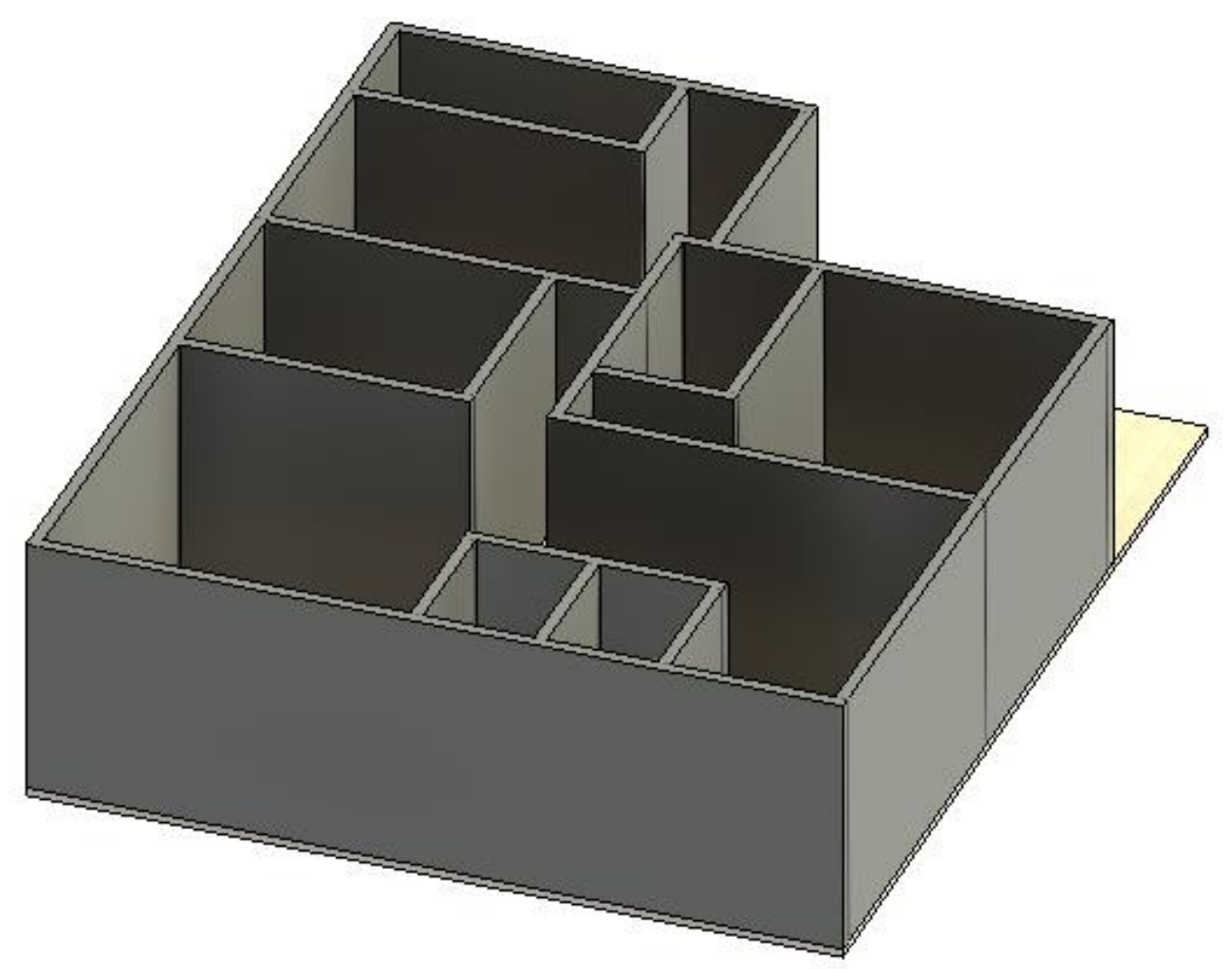

Fonte: Software Revit (2021), 2021 


\section{Pontificia Universidade Católica $_{\text {a }}$ DO RIO DE JANEIRO}

Figura 11: Paredes do térreo após a aplicação da pintura

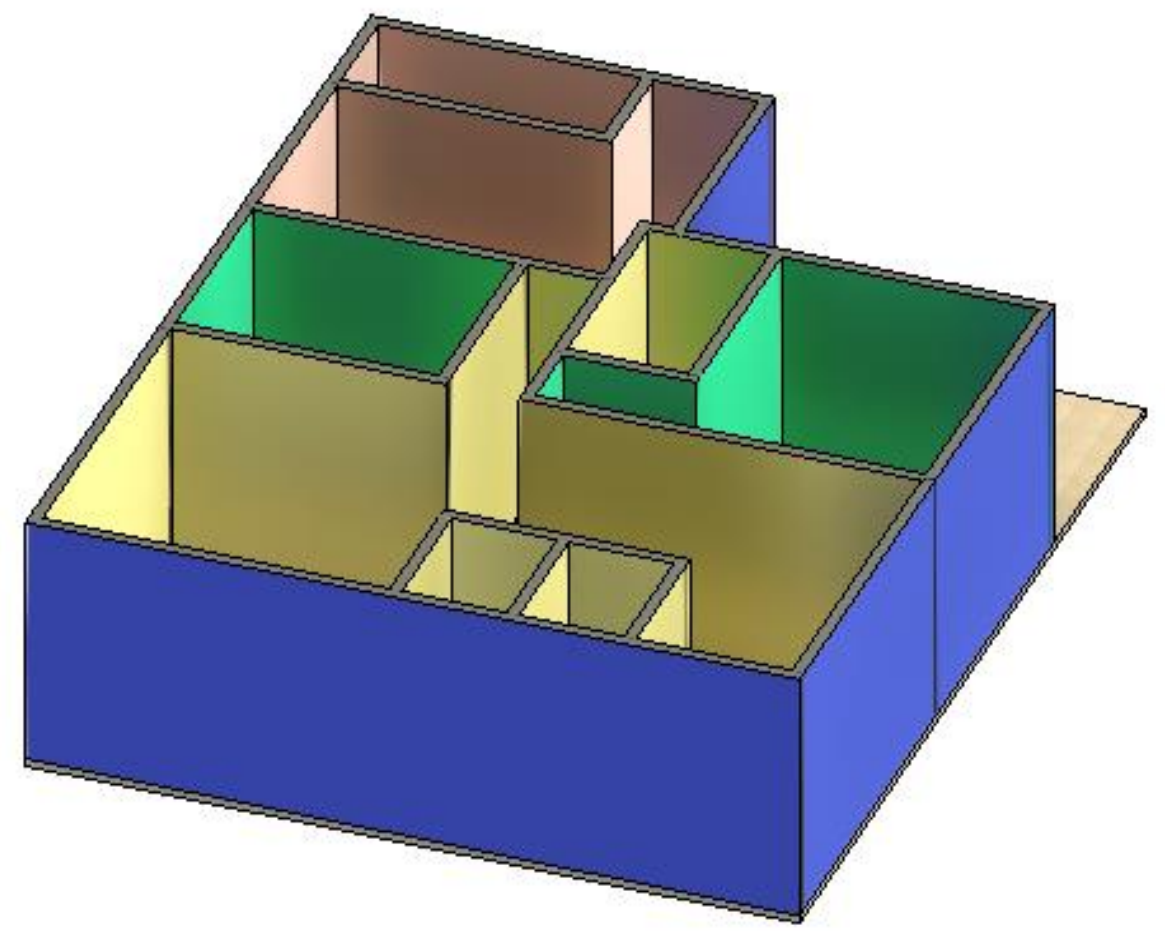

Fonte: Software Revit (2021), 2021 
Figura 12: Paredes externas de toda a edificação após a pintura

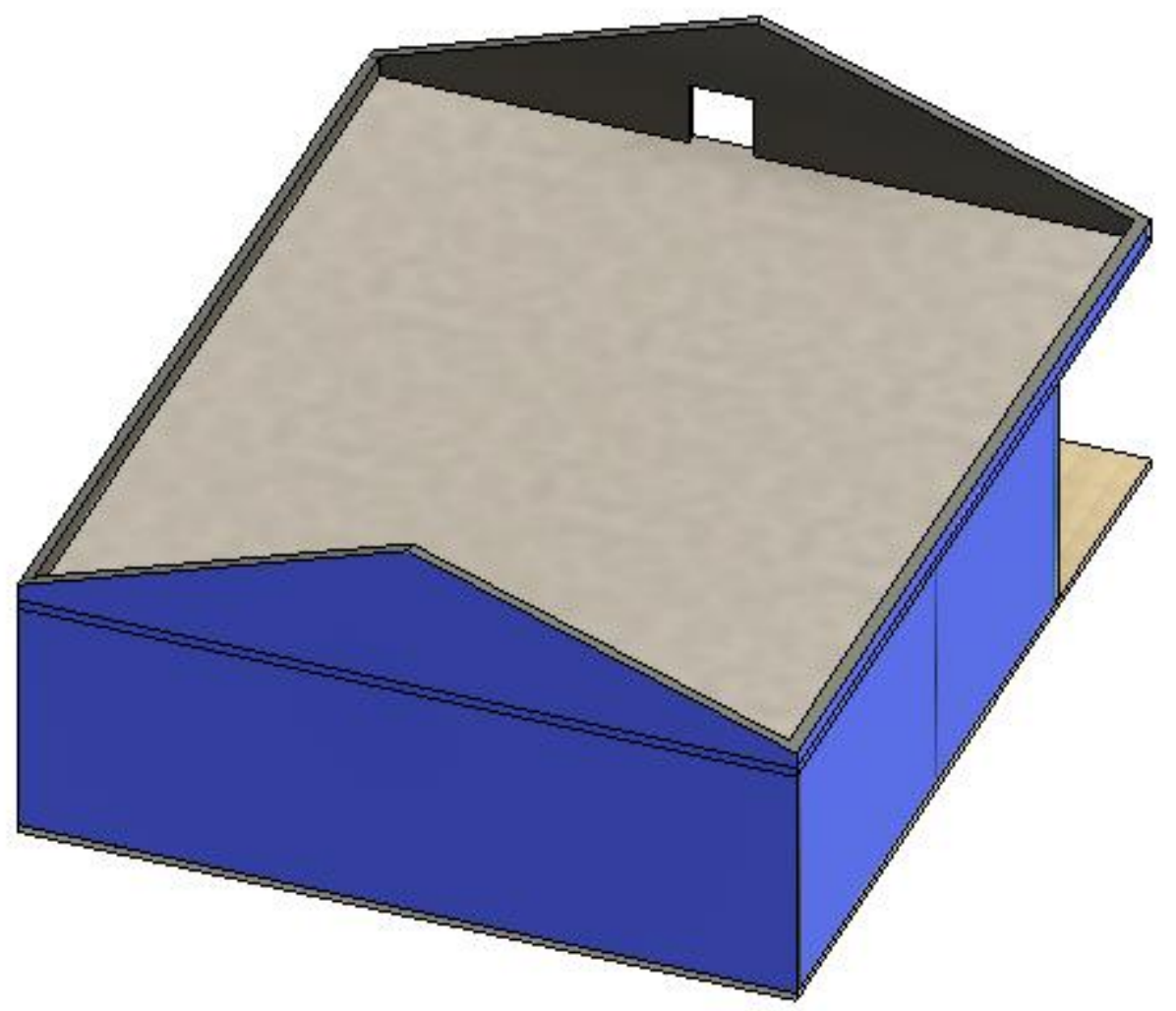

Fonte: Software Revit (2021), 2021

\subsection{Mobiliário e esquadrias}

Após a inserção das paredes, pisos e aplicação da pintura, foi feito a inserção do mobiliário e das esquadrias (figuras 13 e 14), estes no Revit são chamados de famílias (móveis e esquadrias meramente ilustrativos). 


\section{Pontifícia Universidade Católlca

Figura 13: Inserção do mobiliário e esquadrias - 2D

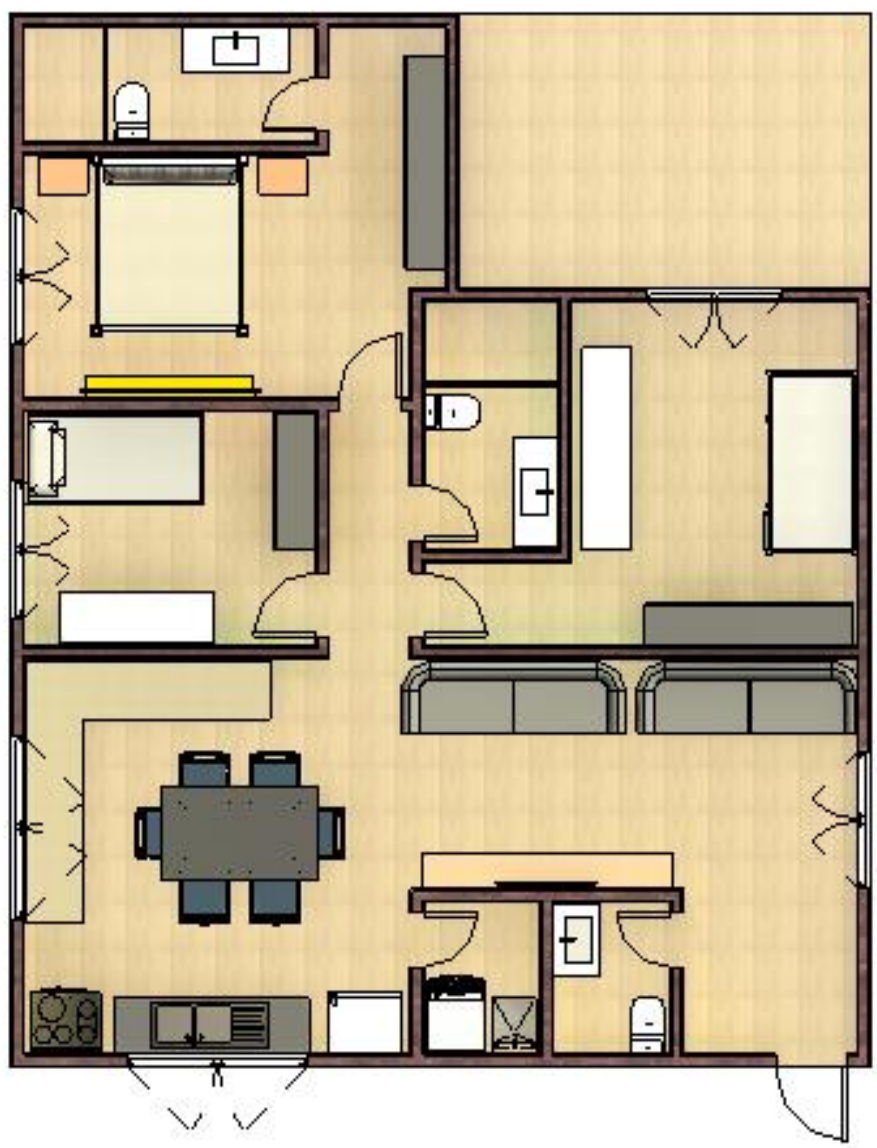

Fonte: Software Revit (2021), 2021 


\section{Pontifícia Universidade Católlica $_{\text {a }}$ \\ DO RIO DE JANEIRO}

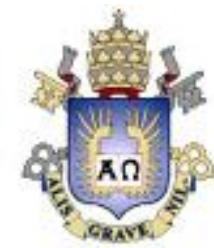

Figura 14: Inserção do mobiliário e esquadrias - 3D

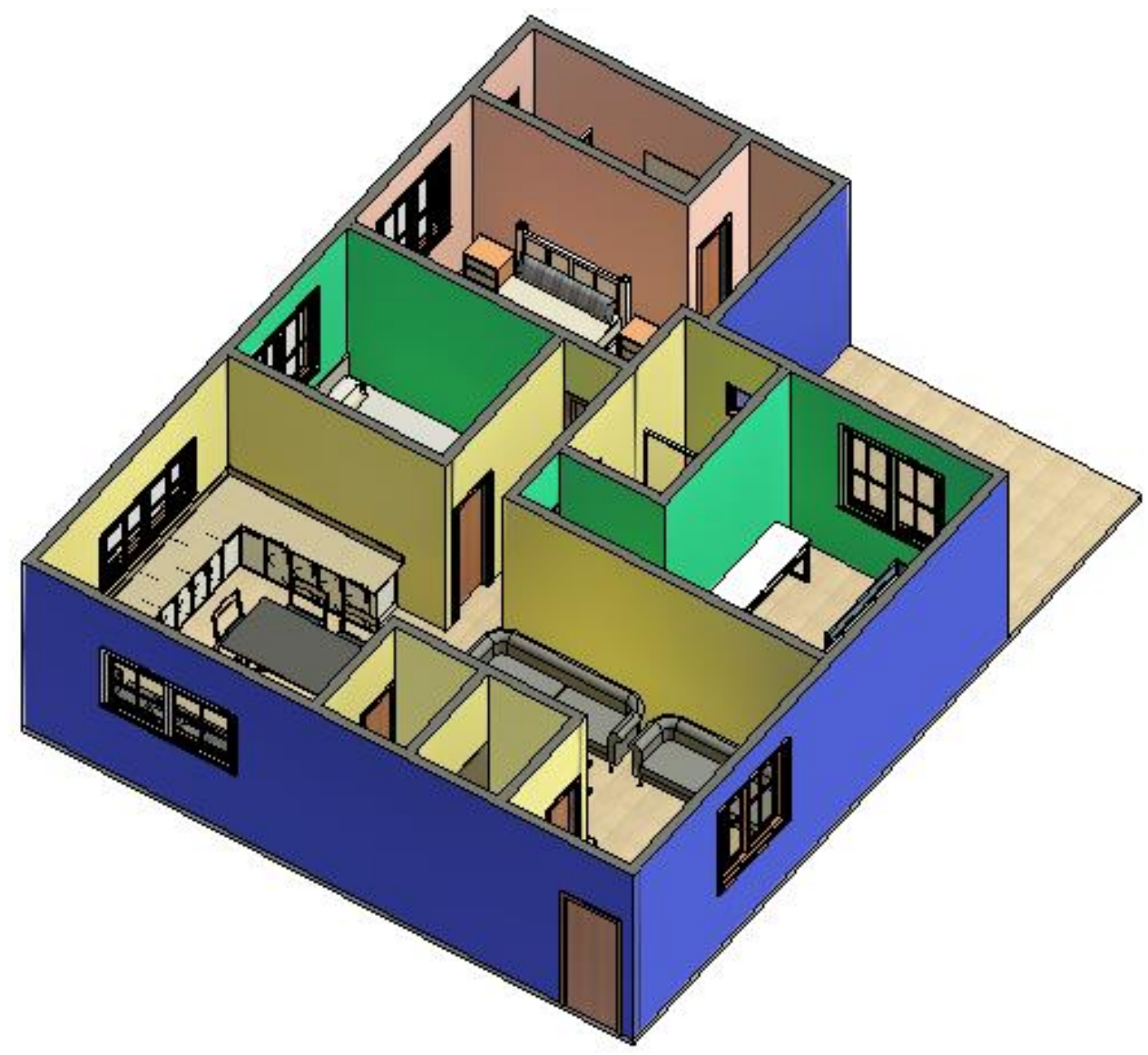

Fonte: Software Revit (2021), 2021

\subsection{Telhado}

Para concluir foi feito a inserção do telhado, este é composto de 2 águas, um beiral de $60 \mathrm{~cm}$ e telha cerâmica (figura 15). 


\section{Pontifícia Universidade Catálica

Figura 15: Telhado

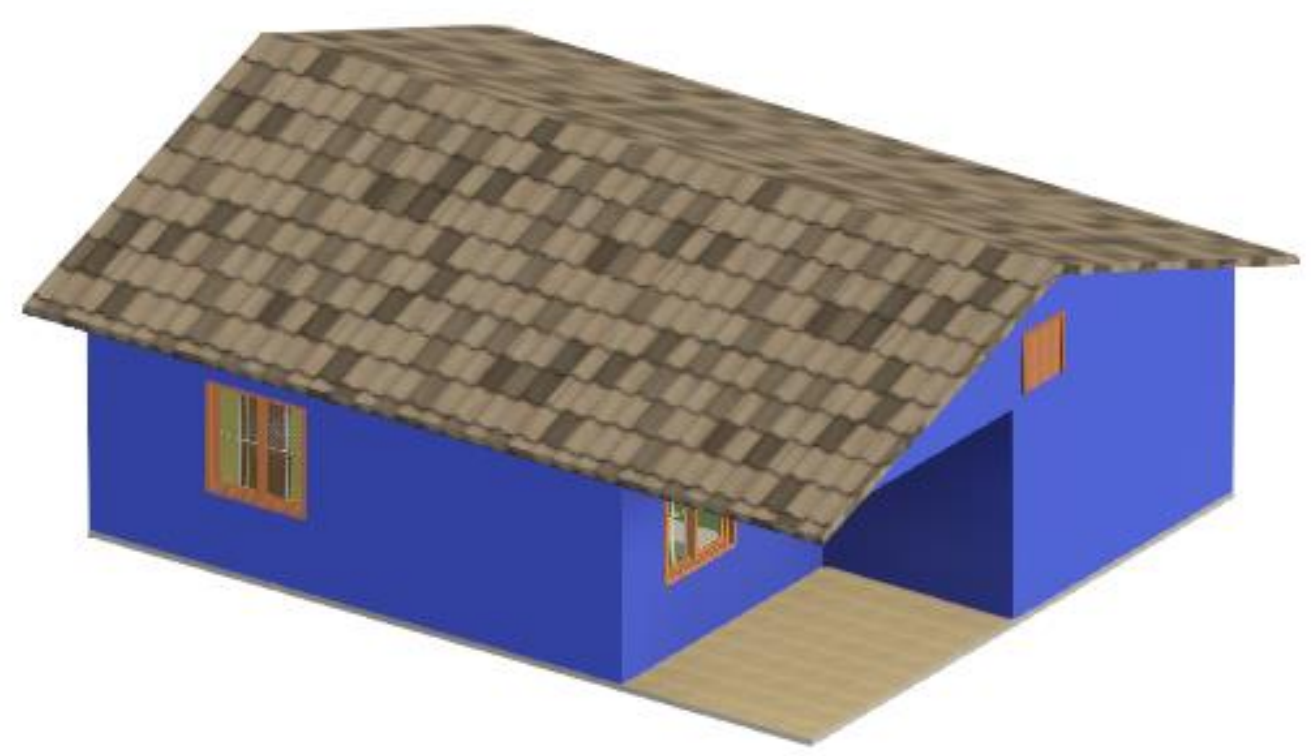

Fonte: Software Revit (2021), 2021

\section{PROJETO ESTRUTURAL}

\subsection{Projeto base e tipo de estrutura}

Para a elaboração do projeto estrutural também foi tomado como base o mesmo croqui mencionado no item 2.1 deste trabalho (ver figura 1 ).

A estrutura será de concreto armado e todo o seu dimensionamento será feito através do software Cypecad.

\subsection{Considerações iniciais}

\subsubsection{Classe da Agressividade Ambiental}

A NBR 6118:2014 afirma que "a agressividade do meio ambiente está relacionada às ações físicas e químicas que atuam sobre as estruturas de concreto, independentemente das ações mecânicas, das variações volumétricas de origem térmica [...]”. 
A edificação encontra-se em área urbana, de acordo com a figura 16, podemos determinar a Classe de Agressividade Ambiental como II (Moderada).

Figura 16 - Classe de Agressividade Ambiental

\begin{tabular}{|c|c|c|c|}
\hline $\begin{array}{c}\text { Classe de } \\
\text { agressividade } \\
\text { ambiental } \\
\end{array}$ & Agressividade & $\begin{array}{l}\text { Classificação geral do tipo } \\
\text { de ambiente para efeito de } \\
\text { projeto }\end{array}$ & $\begin{array}{c}\text { Risco de } \\
\text { deterioração da } \\
\text { estrutura } \\
\end{array}$ \\
\hline \multirow[t]{2}{*}{ 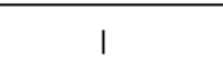 } & \multirow{2}{*}{ Fraca } & Rural & \multirow{2}{*}{ Insignificante } \\
\hline & & Submersa & \\
\hline II & Moderada & Urbana ${ }^{1) 2)}$ & Pequeno \\
\hline \multirow{2}{*}{ III } & \multirow{2}{*}{ Forte } & Marinha ${ }^{1)}$ & \multirow{2}{*}{ Grande } \\
\hline & & Industrial1)2) & \\
\hline \multirow{2}{*}{ IV } & \multirow{2}{*}{ Muito forte } & Industria|11)3) & \multirow{2}{*}{ Elevado } \\
\hline & & Respingos de maré & \\
\hline
\end{tabular}

1) Pode-se admitir um micro-clima com classe de agressividade um nivel mais brando para ambientes internos secos (salas, domitórios, banheiros, cozinhas e áreas de serviço de apartamentos residenciais e conjuntos comerciais ou ambientes com concreto revestido com argamassa e pintura).

2) Pode-se admitir uma classe de agressividade um nivel mais branda em: obras em regiốes de clima seco, com umidade relativa do ar menor ou igual a $65 \%$, partes da estrutura protegidas de chuvas em ambientes predominantemente secos ou regiốes onde chove raramente.

3) Ambientes quimicamente agressivos, tanques industriais, galvanoplastia, branqueamento em industrias de celulose e papel, amazéns de fertilizantes, industrias químicas.

\section{Fonte: NBR 6118:2014}

\subsubsection{Qualidade do Concreto}

Ao determinar a classe de agressividade ambiental a NBR 6118:2014 permite determinar a qualidade do concreto.

De acordo com a classe de agressividade ambiental obtida no item 3.2.1 a classe do concreto deverá ser maior ou igual a C25 e a relação de água/cimento em massa deverá ser menor ou igual a 0,60. Conforme pode ser visto na figura 17 . 


\section{Pontifícia Universidade Catálica}

DO RIO DE JANEIRO

Figura 17 - Relação entre a classe de agressividade e qualidade do concreto

\begin{tabular}{|c|c|c|c|c|c|}
\hline \multirow{2}{*}{ Concreto ${ }^{a}$} & \multirow{2}{*}{ Tipo b,c } & \multicolumn{4}{|c|}{ Classe de agressividade (Tabela 6.1) } \\
\hline & & 1 & "I & III & IV \\
\hline \multirow{2}{*}{$\begin{array}{l}\text { Relação } \\
\text { água/cimento em } \\
\text { massa }\end{array}$} & $C A$ & $\leq 0,65$ & $\leq 0,60$ & $\leq 0,55$ & $\leq 0,45$ \\
\hline & $\mathrm{CP}$ & $\leq 0,60$ & $\leq 0,55$ & $\leq 0,50$ & $\leq 0,45$ \\
\hline \multirow{2}{*}{$\begin{array}{l}\text { Classe de concreto } \\
\text { (ABNTNBR 8953) }\end{array}$} & $C A$ & $\geq \mathrm{C} 20$ & $\geq \mathrm{C} 25$ & $\geq \mathrm{C} 30$ & $\geq C 40$ \\
\hline & $C P$ & $\geq C 25$ & $\geq C 30$ & $\geq C 35$ & $\geq C 40$ \\
\hline \multicolumn{6}{|c|}{$\begin{array}{l}\text { O concreto empregado na execução das estruturas deve cumprir com os requisitos } \\
\text { estabelecidos na ABNT NBR } 12655 \text {. } \\
\text { b CA corresponde a componentes e elementos estruturais de concreto armado. } \\
\text { c CP corresponde a componenfes e elementos estruturais de concreto protendido. }\end{array}$} \\
\hline
\end{tabular}

Fonte: NBR 6118:2014

\subsubsection{Cobrimento nominal}

Segundo a NBR-6118:2014, "para garantir o cobrimento mínimo (Cmin), o projeto e a execução devem considerar o cobrimento nominal (Cnom), que é o cobrimento mínimo acrescido da tolerância de execução $(\Delta c)[\ldots] ”$.

Pela classe de agressividade ambiental é possível determinar o cobrimento nominal mínimo para os diferentes tipos de elementos estruturais, conforme pode ser visto na figura 18 
Figura 18 - Relação entre a classe de agressividade e o cobrimento nominal

\begin{tabular}{|c|c|c|c|c|c|}
\hline \multirow{3}{*}{ Tipo de estrutura } & \multirow{3}{*}{$\begin{array}{l}\text { Componente ou } \\
\text { elemento }\end{array}$} & \multicolumn{4}{|c|}{ Classe de agressividade ambiental (Tabela 6.1) } \\
\hline & & $\mathbf{I}$ & II & III & IV ${ }^{c}$ \\
\hline & & \multicolumn{4}{|c|}{$\begin{array}{l}\text { Cobrimento nominal } \\
\text { mm }\end{array}$} \\
\hline \multirow{3}{*}{ Concreto armado } & Laje b & 20 & 25 & 35 & 45 \\
\hline & Viga/pilar & 25 & 30 & 40 & 50 \\
\hline & $\begin{array}{l}\text { Elementos } \\
\text { estruturais em } \\
\text { contato com o solo d }\end{array}$ & \multicolumn{2}{|c|}{30} & 40 & 50 \\
\hline \multirow{2}{*}{$\begin{array}{l}\text { Concreto } \\
\text { protendido a }\end{array}$} & Laje & 25 & 30 & 40 & 50 \\
\hline & Viga/pilar & 30 & 35 & 45 & 55 \\
\hline \multicolumn{6}{|c|}{$\begin{array}{l}\text { a Cobrimento nominal da bainha ou dos fios, cabos e cordoalhas. } O \text { cobrimento da armadura passiva deve } \\
\text { respeitar os cobrimentos para concreto armado. }\end{array}$} \\
\hline \multicolumn{6}{|c|}{$\begin{array}{l}\text { Para a face superior de lajes e vigas que serão revestidas com argamassa de contrapiso, com revestimentos } \\
\text { finais secos tipo carpete e madeira, com argamassa de revestimento e acabamento, como pisos de } \\
\text { elevado desempenho, pisos cerâmicos, pisos asfálticos e outros, as exigências desta Tabela podem ser } \\
\text { substituídas pelas de 7.4.7.5, respeitado um cobrimento nominal } \geq 15 \mathrm{~mm} \text {. }\end{array}$} \\
\hline \multicolumn{6}{|c|}{$\begin{array}{l}\text { c Nas superfícies expostas a ambientes agressivos, como reservatórios, estaçōes de tratamento de água e } \\
\text { esgoto, condutos de esgoto, canaletas de efluentes e outras obras em ambientes química e intensamente } \\
\text { agressivos, devem ser atendidos os cobrimentos da classe de agressividade IV. }\end{array}$} \\
\hline \multicolumn{6}{|c|}{$\begin{array}{l}\text { do trecho dos pilares em contato com o solo junto aos elementos de fundaçāo, a armadura deve ter } \\
\text { cobrimento nominal } \geq 45 \mathrm{~mm} \text {. }\end{array}$} \\
\hline
\end{tabular}

Fonte: NBR 6118:2014

\subsection{Fundação}

Para uma modelagem inicial, foi definido a cota da fundação a 1 m abaixo do nível do terreno com um solo de característica de argila dura, apenas para uma análise inicial, futuramente será feito uma sondagem SPT para definições do tipo de fundação e ajuste da cota de fundação, caso seja necessário.

\subsection{Modelagem da estrutura no Cypecad}

\subsubsection{Introdução automática dos dados}

O software CYPECAD possui a opção de introdução automática DXF/DWG, conforme pode ser visto na figura 19.

É um assistente para a elaboração do projeto onde serão inseridos os dados dos pavimentos, subsolos (quando houver), altura do pé direito, cargas permanentes como impermeabilização, revestimentos, entre outros e sobrecargas de utilização, fundação além de atribuições de layers (cores definidas para cada camada) para facilitar a visualização, edição e organização do projeto. (ANDRADE, R. 2017). 


\section{Pontifícia Universidade Catálica

Figura 19 - Introdução automática

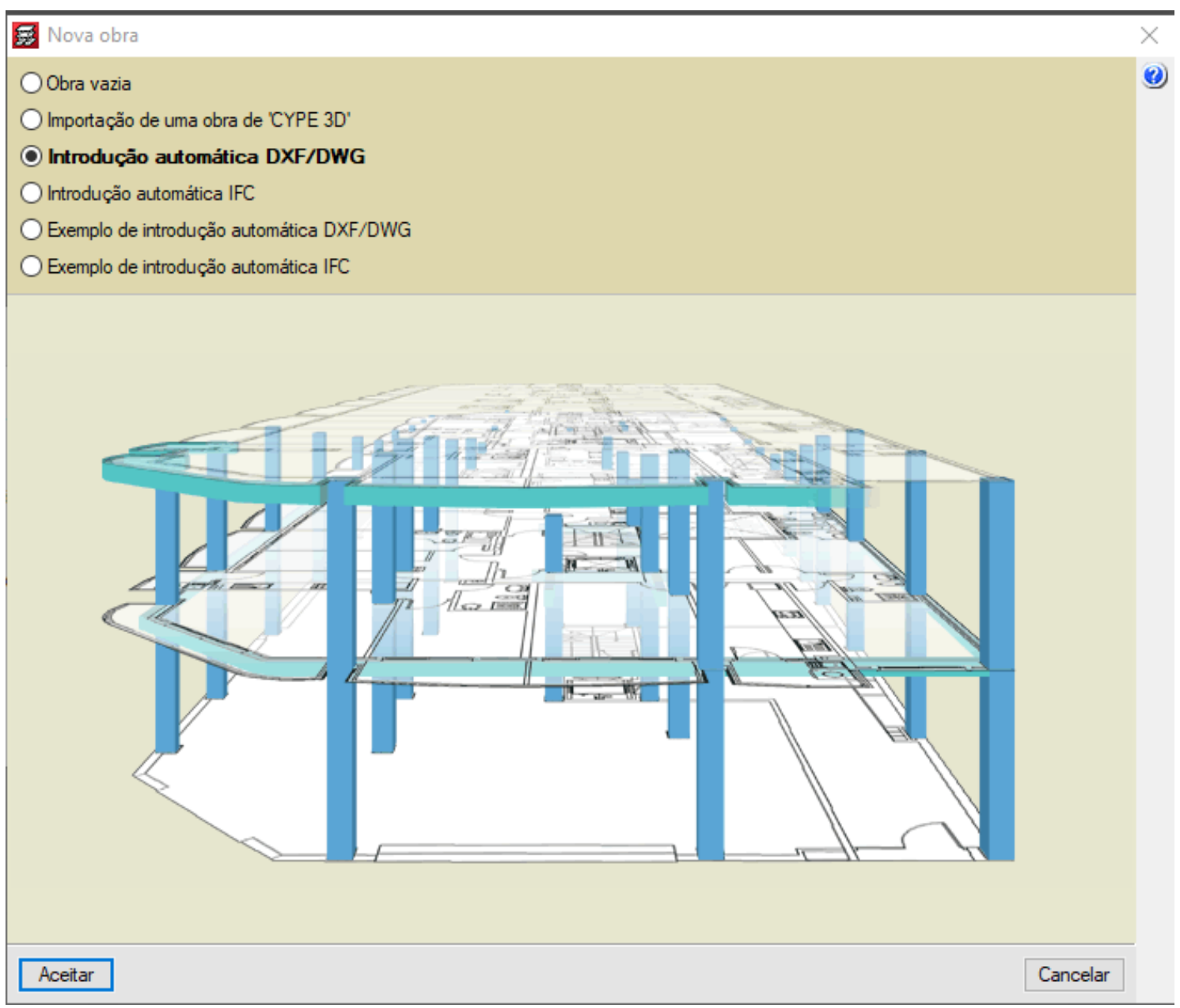

Fonte: Software Cypecad (2017), 2021

\subsubsection{Dados dos grupos e pisos}

Nesta seção, será incorporado alguns dados sobre a edificação. Conforme o projeto arquitetônico, já apresentado, o projeto se trata de uma casa unifamiliar de 1 pavimento. Não possui casa de máquinas e subsolo.

Com isso, é preenchido a tabela (pertence ao software Cypecad) da figura 20, com os dados de: pé direito estrutural, sobrecarga de utilização e carga permanente 


\section{Pontifícia Universidade Catálica $_{\text {a }}$ DO RIO DE JANEIRO}

Figura 20 - Dados da edificação

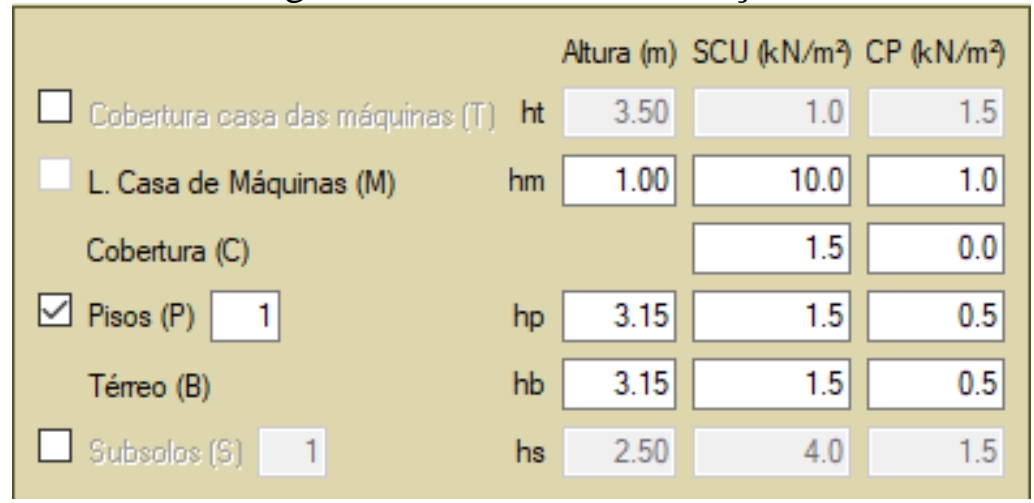

Fonte: Software Cypecad (2017), 2021

Foi adotado um pé-direito de 3,15 m. Os valores de sobrecarga de utilização

(SCU) e carga permanente (CP) estão de acordo com a norma ABNT-6120.

Figura 21 - Descrição dos grupos

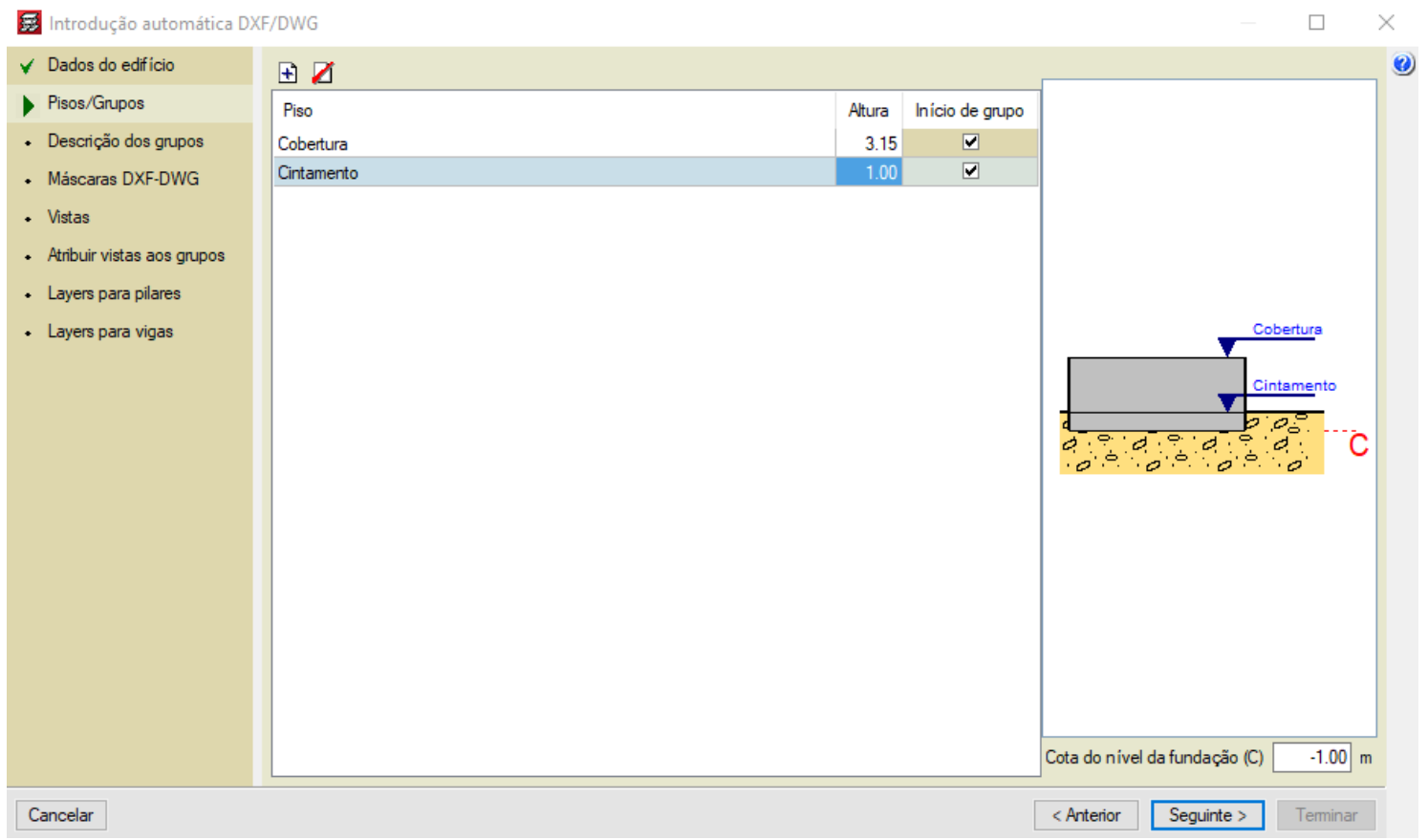

Fonte: Software Cypecad (2017), 2021 


\subsubsection{Máscara DXF-DWG}

Com base no croqui feito no AutoCAD (figura 1), é feito a sua importação para o Cypecad de modo a facilitar a modelagem do projeto.

Segundo (ANDRADE, R. 2017) "Este arquivo será denominado "Máscara" e quaisquer modificações posteriores poderão gerar mudanças na modelagem do Cypecad”.

\subsubsection{Dados Gerais (Materiais utilizados, Fissuras, cobrimento e tensão admissível do solo)}

Nesta seção, Segundo (ANDRADE, R. 2017) “Será preciso determinar os dados gerais do projeto, ou seja, indicar quais tipos de materiais, ações e coeficientes utilizar durante a modelagem do projeto de acordo com a norma ABNT atual”.

A estrutura da edificação será de concreto armado, de acordo com a classe de agressividade ambiental o fck deve ser $\geq 25 \mathrm{MPa}$. Será adotado um fck de 30MPa (Para cada tipo de fck o software oferece 3 opções: usinado, em geral e condição desfavorável. Por questão de segurança foi adotado o pior caso que é em condição desfavorável.) todos os elementos estruturais da edificação. No caso das barras de aço será adotado o aço CA-50, ou seja, com (fyk) em 500MPa conforme mostra a figura 22.

Segundo (ANDRADE, R. 2017) "Para os elementos estruturais da edificação, o Cypecad determina um valor de abertura máxima de fissuração e cobrimento de acordo com a norma NBR-6118:2014 através da indicação da classificação da CAA”, conforme a figura 23.

O Cypecad já sugere valores de tensão admissível por tipo de solo, sendo que esses valores podem ser editáveis. De acordo com item 3.3 (Fundação) está sendo considerado um solo de argila dura, os valores das tensões podem ser vistos na figura 24. Segundo (ANDRADE, R. 2017) "S1 (MPa) são os valores de tensão admissível no 


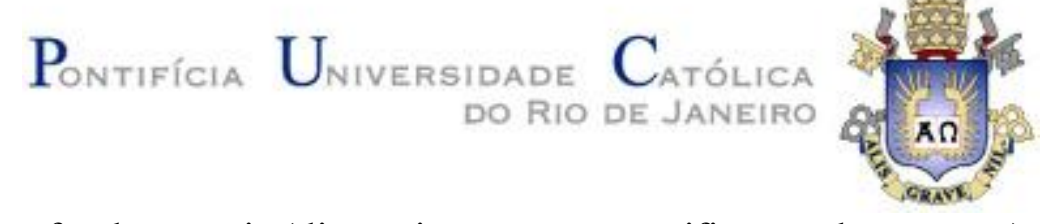

terreno para as combinações fundamentais (dimensionamento e verificação das sapatas) e S2 (MPa) são para combinações sísmicas e acidentais".

Figura 22 - Especificações de concreto e Aço

\begin{tabular}{|c|c|c|c|}
\hline Concreto armado & & & $\mathrm{fc}$ \\
\hline \multicolumn{4}{|l|}{ Concreto } \\
\hline Pisos & C30, con desfav. $\vee$ & EA & \\
\hline Fundação & C30, con desfav. $\vee$ & 坓 & \\
\hline Tubulões & C20, em geral $\quad \checkmark$ & & \\
\hline Pilares & C30, con desfav. $\vee$ & EA & \\
\hline Cortinas & C20, em geral $\quad \checkmark$ & 88 & 臣 \\
\hline Caracteristicas do agregado & Granito $(15 \mathrm{~mm})$ & & \\
\hline \multicolumn{4}{|l|}{ Aço } \\
\hline Barras & CA- -50 e CA- $60 \quad \checkmark$ & 䖽 & \\
\hline Parafusos & ISO 898.C4.6 & 四 & \\
\hline
\end{tabular}

Fonte: Software Cypecad (2017), 2021

Figura 23 - Fissuras e cobrimento

: Ambiente - Vigas $\quad \times$

\begin{tabular}{l} 
Agressividade do ambiente \\
OCAA I: Agressividade fraca \\
OCAA II: Agressividade moderada \\
OCAA III: Agressividade forte \\
OCAA IV: Agressividade muito forte \\
\hline Fissuração \\
Abertura máxima de fissura $0.30 \mathrm{~mm}$
\end{tabular}

Fonte: Software Cypecad (2017), 2021 
Figura 24 - Tensões admissíveis no solo de apoio

: Importar valores usuais de projeto

\begin{tabular}{|l|l|l|}
\hline Tipo de solo & S1 (MPa) & S2 (MPa) \\
\hline Brita & 0.294 & 0.441 \\
\hline Areia densa & 0.245 & 0.368 \\
\hline Areia semidensa & 0.196 & 0.294 \\
\hline Areia solta & 0.098 & 0.147 \\
\hline Lodo & 0.118 & 0.177 \\
\hline Argila dura & 0.245 & 0.368 \\
\hline Argila semi-dura & 0.147 & 0.221 \\
\hline Argila branda & 0.098 & 0.147 \\
\hline
\end{tabular}

Fonte: Software Cypecad (2017), 2021

\subsubsection{Lançamento dos Pilares}

Após a geração dos dados é feito o lançamento dos pilares (figura 25). Nesta etapa também é feito o ajuste de sua altura, todos nascem na fundação, mas nem todos morrem na laje de cobertura (figura 26). Os pilares P4 (figura 27), P9 e P13 morrem na laje do térreo (nível cintamento), os demais pilares morrem na laje de cobertura. 


\section{Pontifícia Universidade Católlca

Figura 25 - Lançamento dos Pilares

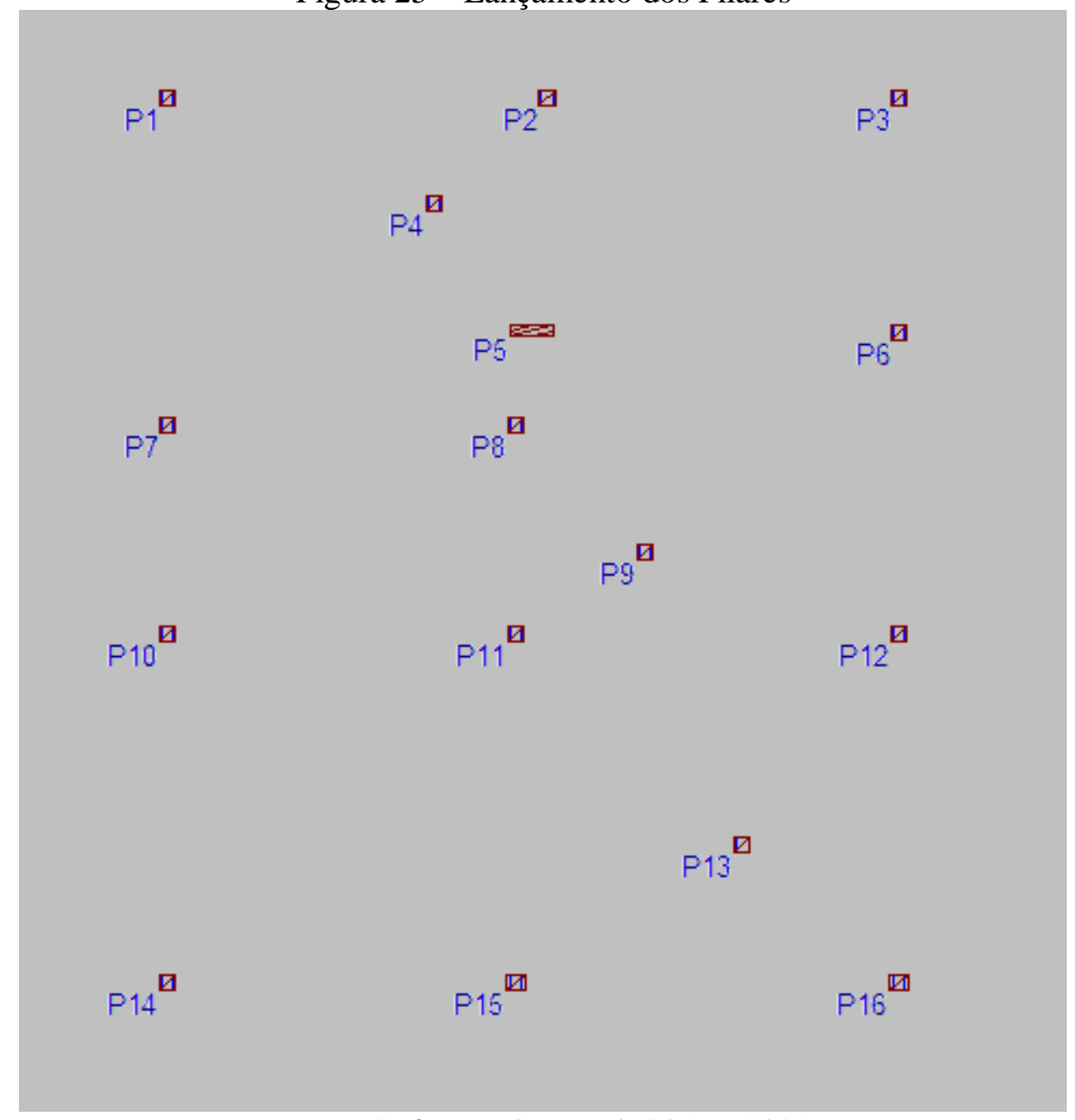

Fonte: Software Cypecad (2017), 2021

Figura 26 - Pilar P1, morre na laje de cobertura

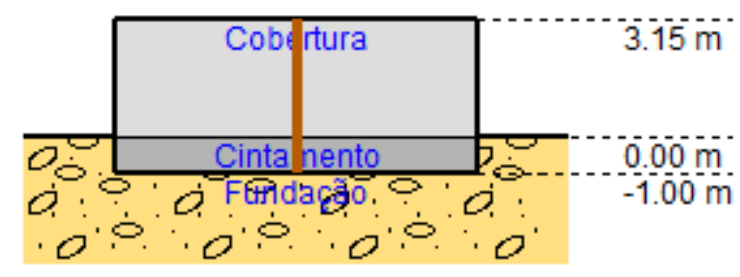

Fonte: Software Cypecad (2017), 2021 
Figura 27 - Pilar P4, morre na laje do térreo (cintamento)

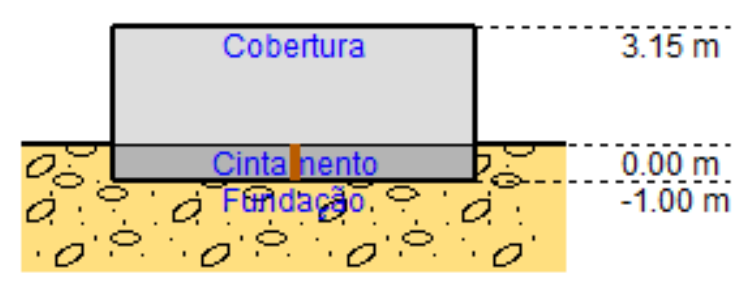

Fonte: Software Cypecad (2017), 2021

\subsubsection{Lançamento e Pré-dimensionamento das vigas}

Para o lançamento e pré-dimensionamento das vigas, foi considerado uma espessura de $15 \mathrm{~cm}$ para todas as vigas/cintas e uma altura de aproximadamente $10 \%$ do vão.

O software Cypecad apresenta diferentes tipos de viga (figura 28), para este projeto foi considerado as do tipo alta e retangular.

As vigas foram lançadas tanto no nível de cintamento (figura 29) quanto no nível da laje de cobertura (figura 30). 
Pontifícia Universidade Católica $_{\text {a }}$ DO RIO DE JANEIRO

Figura 28 - Tipos de viga

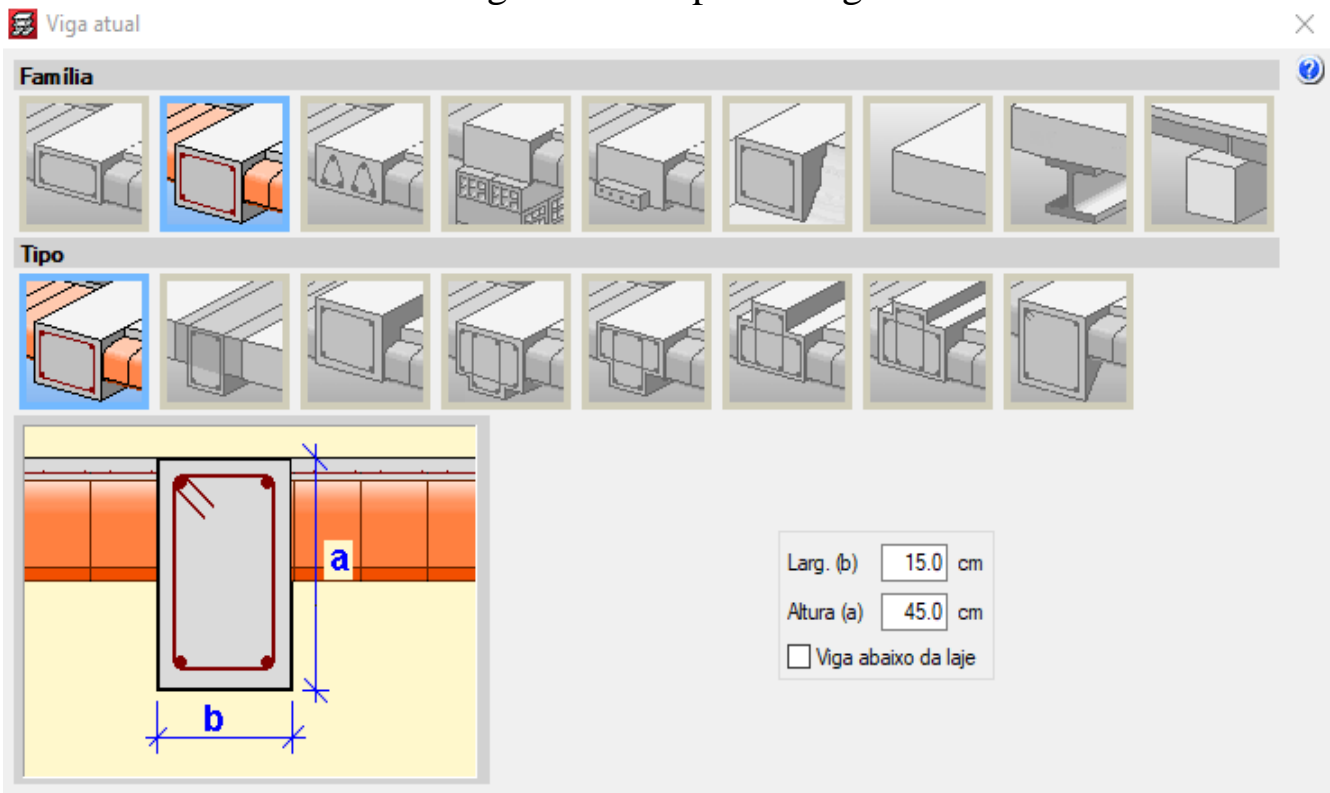

Fonte: Software Cypecad (2017), 2021 


\section{Pontifícia Universidade Católlca DO RIO DE JANEIRO}

Figura 29 - Vigas do nível cintamento

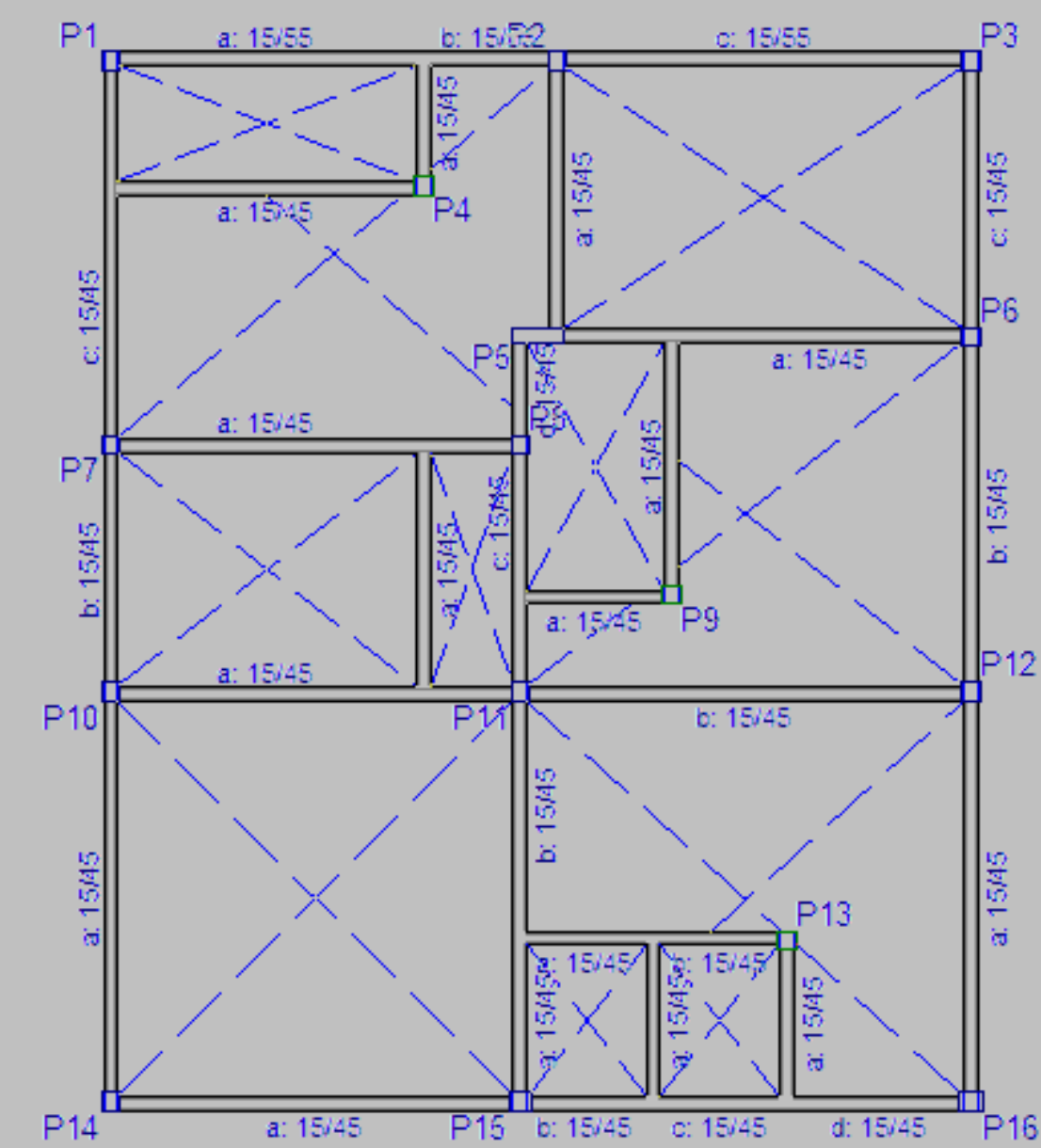

Fonte: Software Cypecad (2017), 2021 
Figura 30 - Vigas do nível cobertura

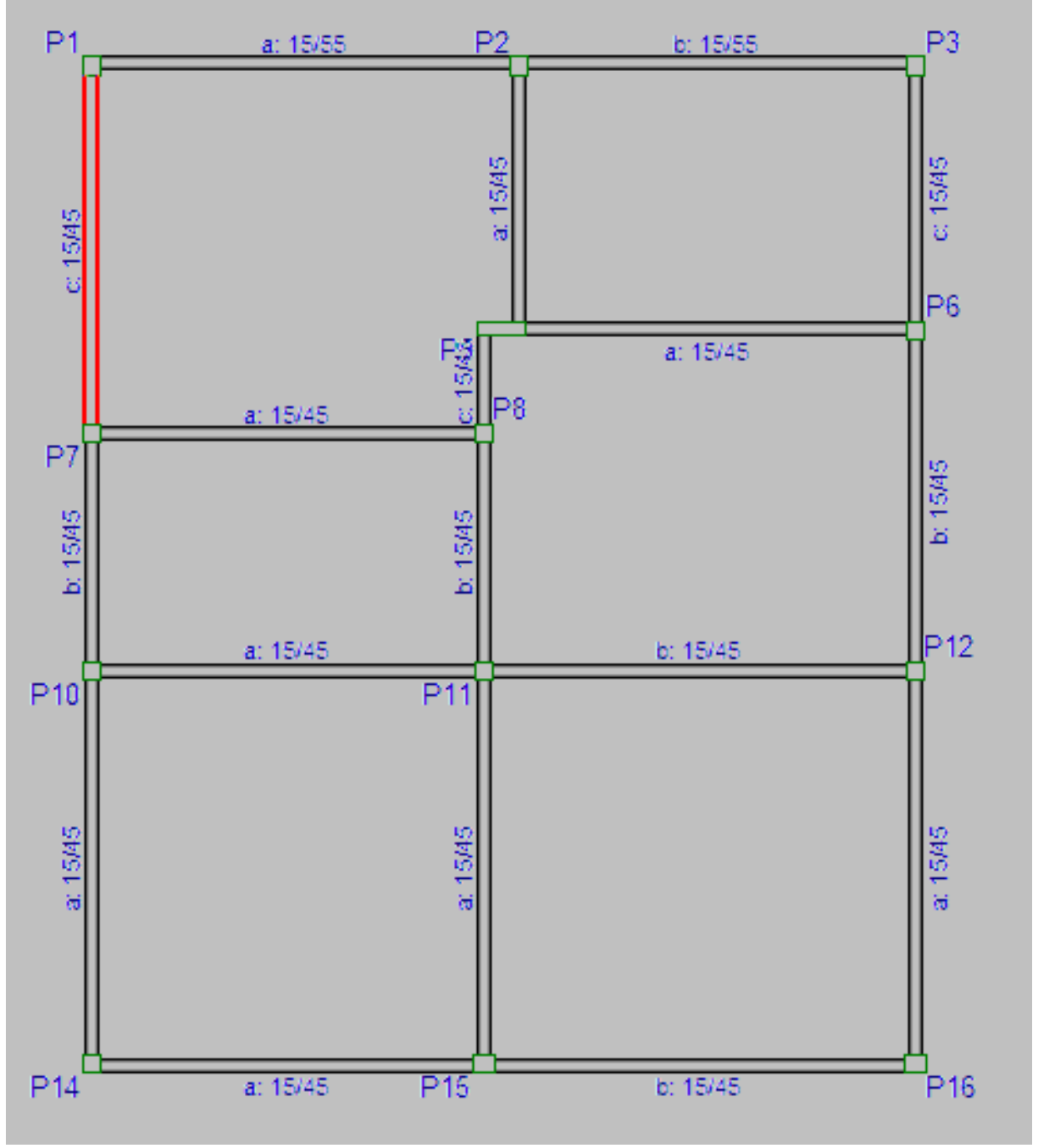

Fonte: Software Cypecad (2017), 2021

\subsubsection{Lançamento e Pré-dimensionamento das lajes}

A laje de cobertura será do tipo pré-moldada com as vigotas posicionadas paralelas ao menor vão. No nível do cintamento não haverá laje (apenas uma camada de regularização de $10 \mathrm{~cm}$, conforme visto no projeto arquitetônico) pois está apoiado diretamente no solo, ou seja, a carga não está sendo transferida para as cintas.

Para a altura da laje do nível da cobertura foi adotado a relação $\mathrm{h}>1 \mathrm{x} / 40$ sendo $1 \mathrm{x}$ a medida do menor vão, com isso foi considerado uma altura de $12 \mathrm{~cm}$ para a laje da cobertura. 
No caso das lajes pré-moldadas, este projeto usará como referência o catálogo da empresa Engemolde, para dimensionamento das vigotas.

Neste catálogo (figura 31) podemos observar que para uma sobrecarga de $2 \mathrm{kN} / \mathrm{m}^{2}$ a laje treliçada do tipo LT12 pode ser aplicada em um vão de até 4,84 m. Neste projeto a sobrecarga é de $1,5 \mathrm{kN} / \mathrm{m}^{2}$ (ver figura 20) e nenhum dos vãos é maior que 4,84 m, logo os valores estão respeitando o solicitado.

Figura 31 - Vãos máximos para lajes treliçadas

\begin{tabular}{|c|c|c|c|c|c|c|c|c|c|c|c|c|c|c|c|c|c|c|c|c|c|}
\hline \multirow[t]{3}{*}{ TIPO } & \multirow{2}{*}{\multicolumn{7}{|c|}{ 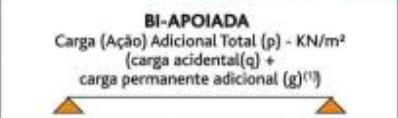 }} & \multirow{2}{*}{\multicolumn{7}{|c|}{ 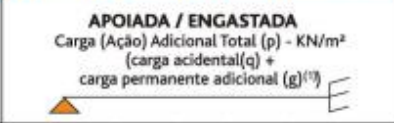 }} & \multicolumn{7}{|c|}{ 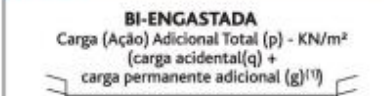 } \\
\hline & & & & & & & & & & & & & & & \multirow[b]{2}{*}{10} & & \multirow[b]{2}{*}{2,5} & \multirow[b]{2}{*}{3,5} & \multirow[b]{2}{*}{5,0} & \multicolumn{2}{|c|}{ L } \\
\hline & 1,0 & 2,0 & 2,5 & 3,5 & 5,0 & 7,5 & 10,0 & 1,0 & 2,0 & 2,5 & 3,5 & 5,0 & 7,5 & 10,0 & & 2,0 & & & & 7,5 & 10,0 \\
\hline LT $10(7+3)$ & 4,30 & 4,10 & 4,01 & 3,67 & 3,18 & 2,68 & 2,41 & 4,92 & 4,67 & 4,56 & 3,95 & 3,41 & 2,85 & 2,54 & 5,37 & 5,09 & 4,86 & 4,49 & 3,85 & 3,21 & 2,66 \\
\hline LT $11(7+4)$ & 4,56 & 4,37 & 4,28 & 4,04 & 3,50 & 2,95 & 2,65 & 5,18 & 4,95 & 4,84 & 4,36 & 3,76 & 3,14 & 2,81 & 5,55 & 5,30 & 5,19 & 4,61 & 3,97 & 3,30 & 2,94 \\
\hline LT $11(8+3)$ & 4,78 & 4,57 & 4,47 & 4,19 & 3,64 & 3,06 & 2,75 & 5,40 & 5,15 & 5.04 & 4,52 & 3.90 & 3,26 & 2,91 & 5,79 & 5,51 & 5,39 & 4,79 & 4,12 & 3,43 & 3,06 \\
\hline LT $12(8+4)$ & 5,04 & 4,84 & 4,75 & 4,58 & 3,97 & 3,35 & 3,01 & 5,69 & 5,44 & 5,34 & 4,95 & 4,27 & 3,58 & 3,20 & 6,00 & 5,83 & 5,71 & 5,26 & 4,53 & 3,77 & 3,36 \\
\hline LT $16(12+4)$ & 6,02 & 6,01 & 6,00 & 5,85 & 5,30 & 4,47 & 4,01 & 6,76 & 6,52 & 6,41 & 6.21 & 5,75 & 4,82 & 4,31 & 7,26 & 7,00 & 6,88 & 6,66 & 6,00 & 5,12 & 4,58 \\
\hline LT $20(16+4)$ & 7,17 & 6,95 & 6,85 & 6.67 & 6,28 & 5,59 & 5,02 & 8,02 & 7,76 & 7,65 & 7,44 & 6,88 & 6,00 & 5,43 & 8,60 & 8,33 & 8,21 & 7,98 & 7,36 & 6,16 & 5,78 \\
\hline LT $25(20+5)$ & 8,45 & 8,24 & 8,14 & 7,95 & 7,70 & 6,66 & 6,00 & 9,40 & 9,15 & 9,04 & 8,83 & 8,54 & 7,27 & 6,52 & 10,00 & 9,87 & 9,74 & 9,51 & 9,20 & 7,78 & 6,97 \\
\hline
\end{tabular}

Fonte: Empresa Engemolde 


\section{Pontifícia Universidade Catálica

Figura 32 - Criação do bloco para lajes pré-moldada

: Editar - [Laje de vigotas de concreto]

Referência

Geometria

Espessura camada de compressão (a)

Attura do bloco/molde (b)

$4 \mathrm{~cm}$

Largura da nervura (d)

$15 \mathrm{~cm}$

Entre eixos (c) $38 \mathrm{~cm}$

Largura longitudinal

Incremento da largura da nervura $2.0 \mathrm{~cm}$

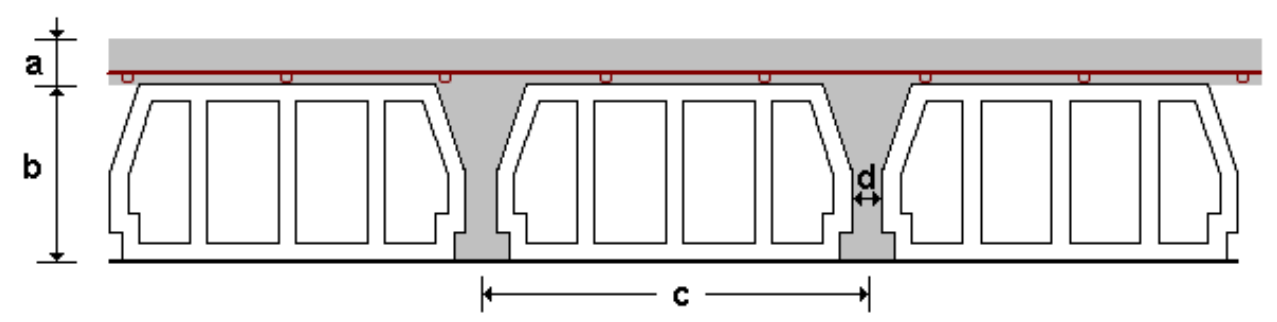

Fonte: Software Cypecad (2017), 2021

Após a inserção desses dados é feito o lançamento da pré-laje no nível da cobertura (figura 33). 


\section{Pontif́́cia Universidade Chá́lica

Figura 33 - Lançamento da pré-laje no nível de cobertura

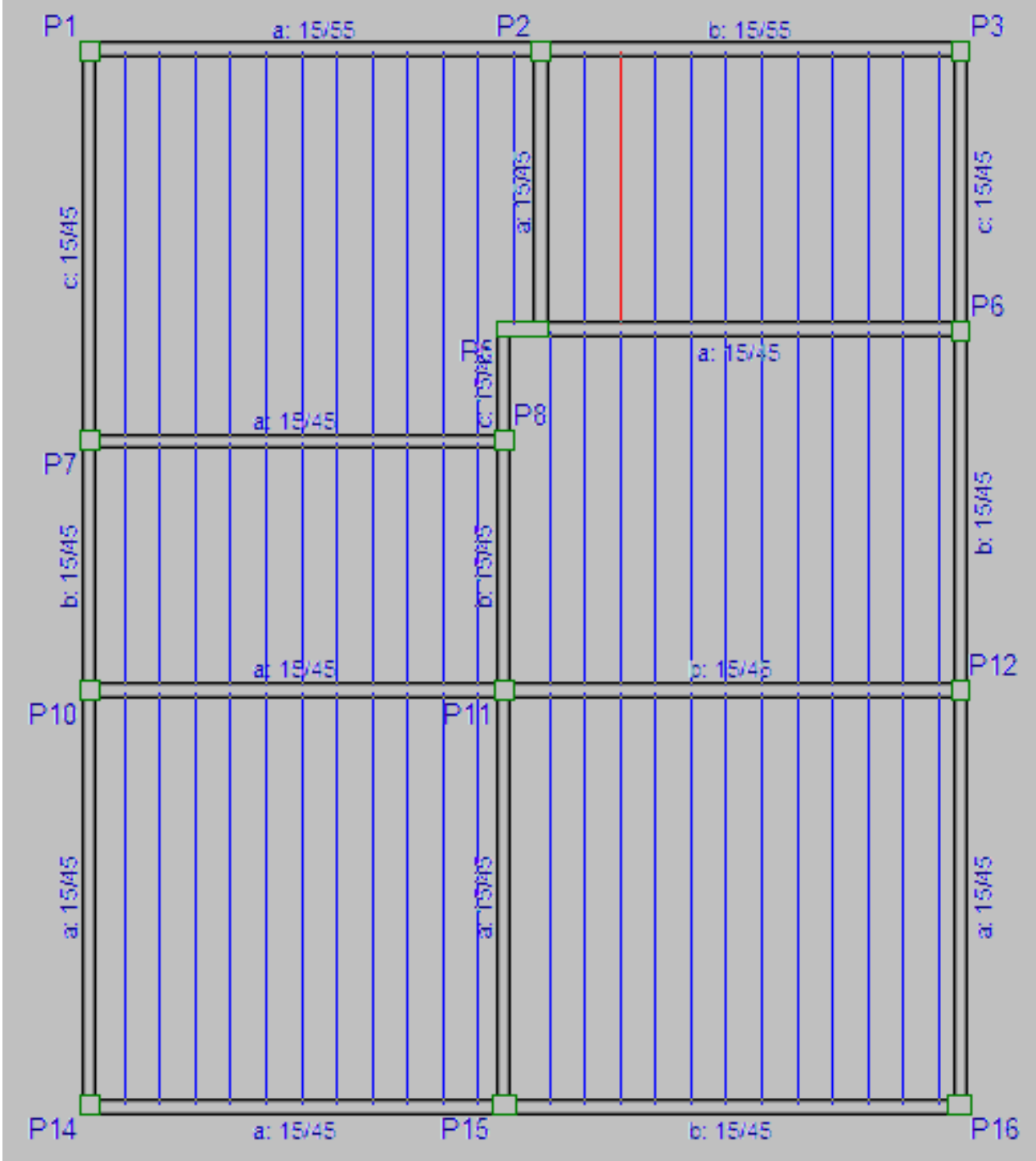

Fonte: Software Cypecad (2017), 2021

\subsubsection{Carregamentos}

No ítem 3.4.2 (figura 20) foram introduzidas às cargas de sobrecarga de utilização (SCU) e carga permanente (CP) nos pisos. Agora, para completar, será introduzido as cargas de alvenaria da edificação e a carga da caixa d'água

Segundo (ANDRADE, R. 2017) "Para as cargas de alvenaria foi levado em consideração a altura do pé direito (desconsiderando a altura da viga ou laje), a espessura (e) da parede (tijolo + reboco) e o peso específico $(\gamma)$ do tijolo". 


\section{Pontifícia Universidade C $\operatorname{tá}$ lóca \\ DO RIO DE JANEIRO}

Segundo (ANDRADE, R. 2017) "Usa-se se a formula: Qalv =e . h . $\gamma$, onde o carregamento da alvenaria (Qalv) é igual à espessura da parede (e), cujo valor multiplica a altura da alvenaria (h) e o peso especifico do tijolo $(\gamma)$ ".

Com a fórmula vista acima, adotou-se $0,15 \mathrm{~m}$ (de acordo com o projeto arquitetônico) para a espessura de todas as paredes. Em toda a edificação será utilizado o tijolo furado, logo se adotou o valor de $13 \mathrm{kN} / \mathrm{m}^{3}$ para o peso específico, seguindo a recomendação da ABNT NBR 6120. Como, de início, o menor valor de altura para as vigas é de $45 \mathrm{~cm}$ e o pé-direito é de $3,15 \mathrm{~m}$, a altura de alvenaria, no térreo, corresponde a 2,70 m (isto corresponde a maior altura de alvenaria que termina em viga e consequentemente a maior carga) com isso a minha carga de alvenaria que termina na

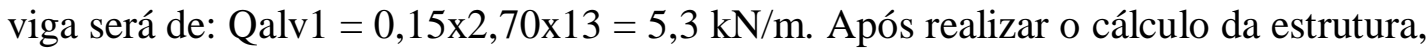
será verificado se há necessidade de mudar o valor desta carga visto que ao alterar a altura da viga poderá ter uma alteração no valor desta carga. Para as paredes que terminam na laje $(\mathrm{h}=0,12 \mathrm{~m})$ foi realizado o mesmo procedimento, neste caso a altura de alvenaria será de 3,15-0,12=3,03 m. Logo, a carga de alvenaria das paredes que terminam na laje de cobertura é de: Qalv2 $=0,15 \times 3,03 \times 13=5,9 \mathrm{kN} / \mathrm{m}$. A aplicação destas cargas pode ser vista na figura 34

Na cobertura há 2 paredes, triangulares, com altura de $2 \mathrm{~m}$, que irão servir de apoio para o telhado, sua carga foi calculada considerando uma parede retangular de $1 \mathrm{~m}$ (metade da altura), assim temos: Qalv3 $=0,15 \times 1,00 \times 13=1,95 \mathrm{kN} / \mathrm{m}$ (conforme pode ser visto na figura 35).

A edificação irá contar com uma caixa d'água de $20001=2,0 \mathrm{t}=20 \mathrm{kN}$. A caixa d'água possui um diâmetro de 1,5 $\mathrm{m}$ logo sua área é $\pi \mathrm{x}(1,5)^{2} / 4=1,76 \mathrm{~m}^{2}$. Logo, sua carga é de Qcx. = 20/1,76 = 11,4 kN/m² (conforme pode ser visto na figura 35). 


\section{Pontifícia Universidade Catálica $_{\text {a }}$}

Figura 34 - Cargas de alvenaria sobre as vigas do cintamento

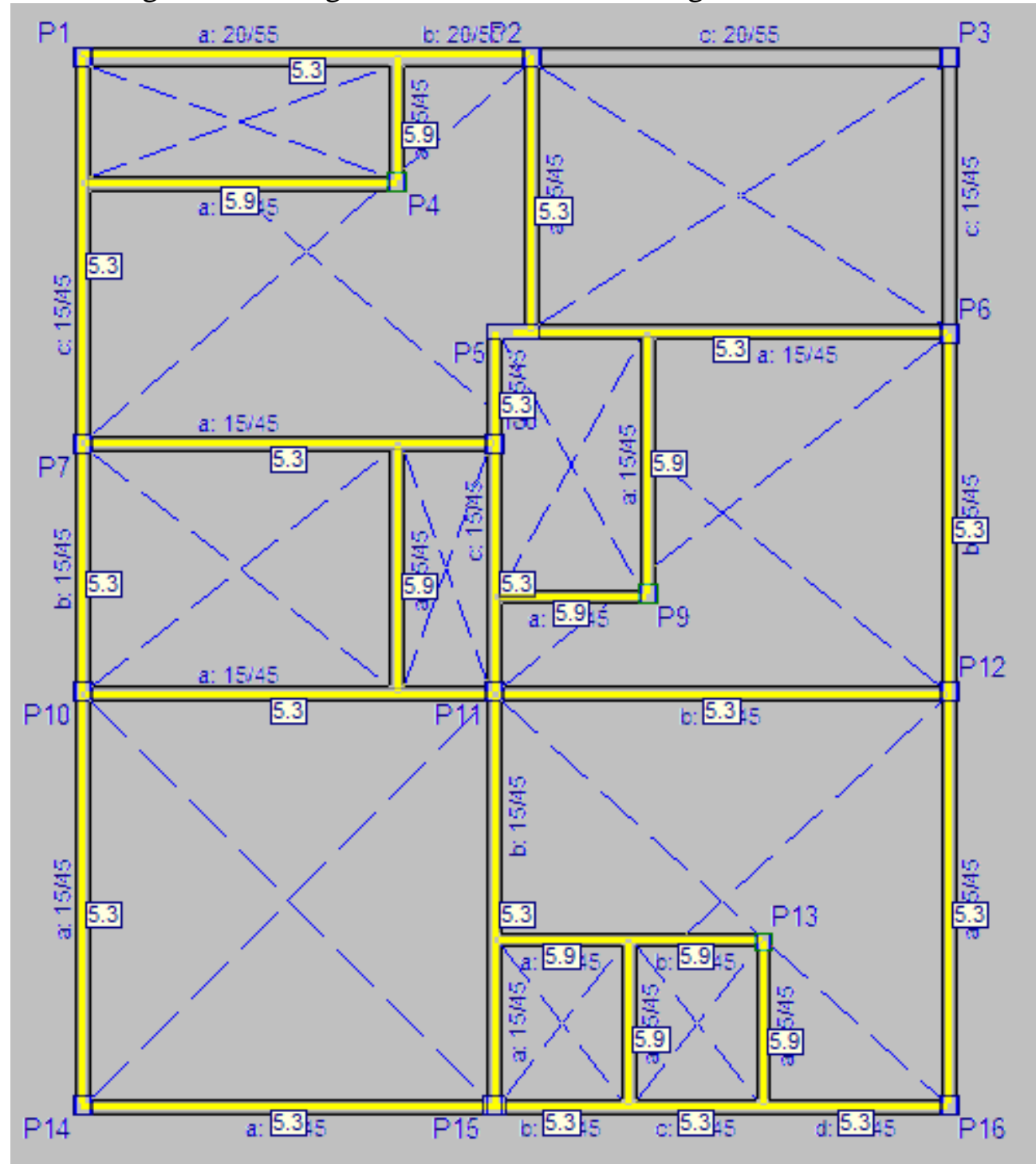

Fonte: Software Cypecad (2017), 2021 


\section{Pontifícia Universidade Catálica $_{\text {a }}$}

Figura 35 - Cargas de alvenaria e caixa d'água no nível da cobertura

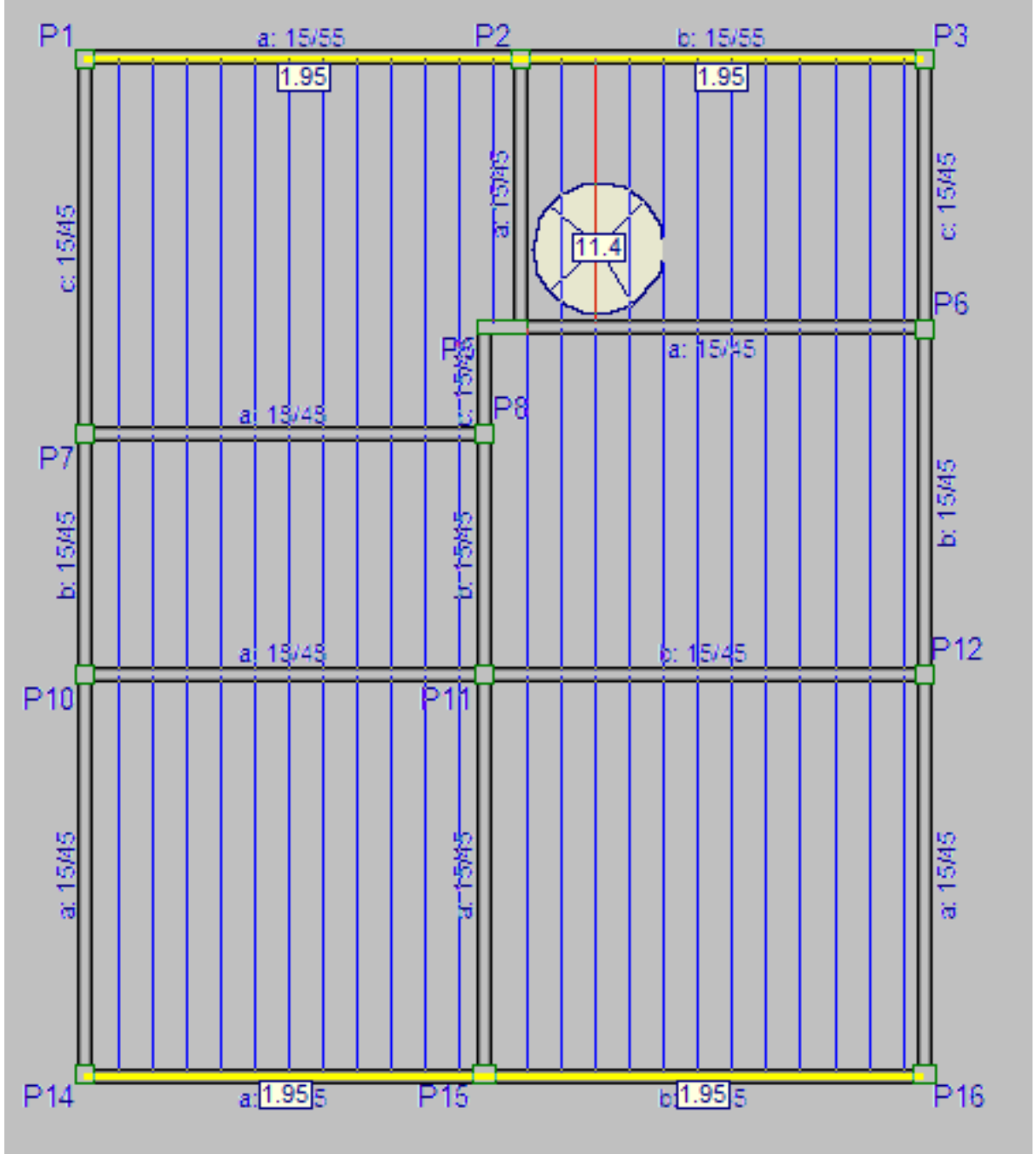

Fonte: Software Cypecad (2017), 2021

\subsubsection{Lançamento da fundação}

Segundo (ANDRADE, R. 2017) “O Cypecad permite três tipos de fundações (sapatas de concreto armado, concreto simples e blocos para estaqueamento) mostrando suas variações quanto ao modelo" (figura 36). Para este projeto, foram adotadas as sapatas de concreto armado do tipo quadrada piramidal. 


\section{Pontifícia Universidade Catálica

Figura 36 - Seleção dos elementos de fundação

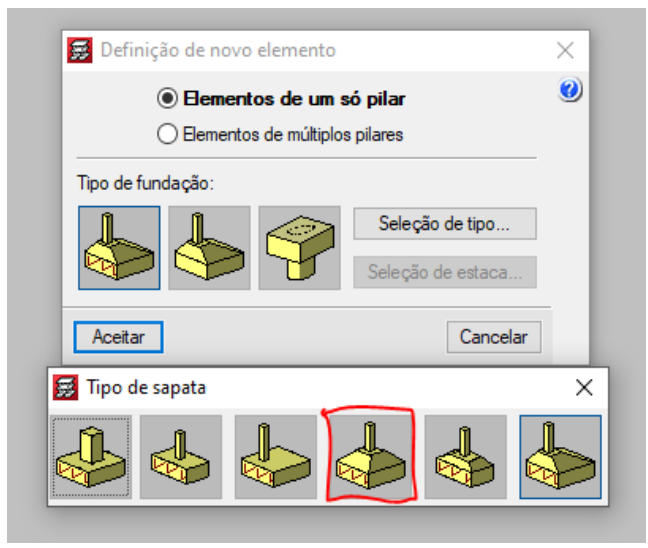

Fonte: Software Cypecad (2017), 2021

Definindo o tipo de fundação, é feito o lançamento no software (figura 37)

Figura 37 - Lançamento da fundação

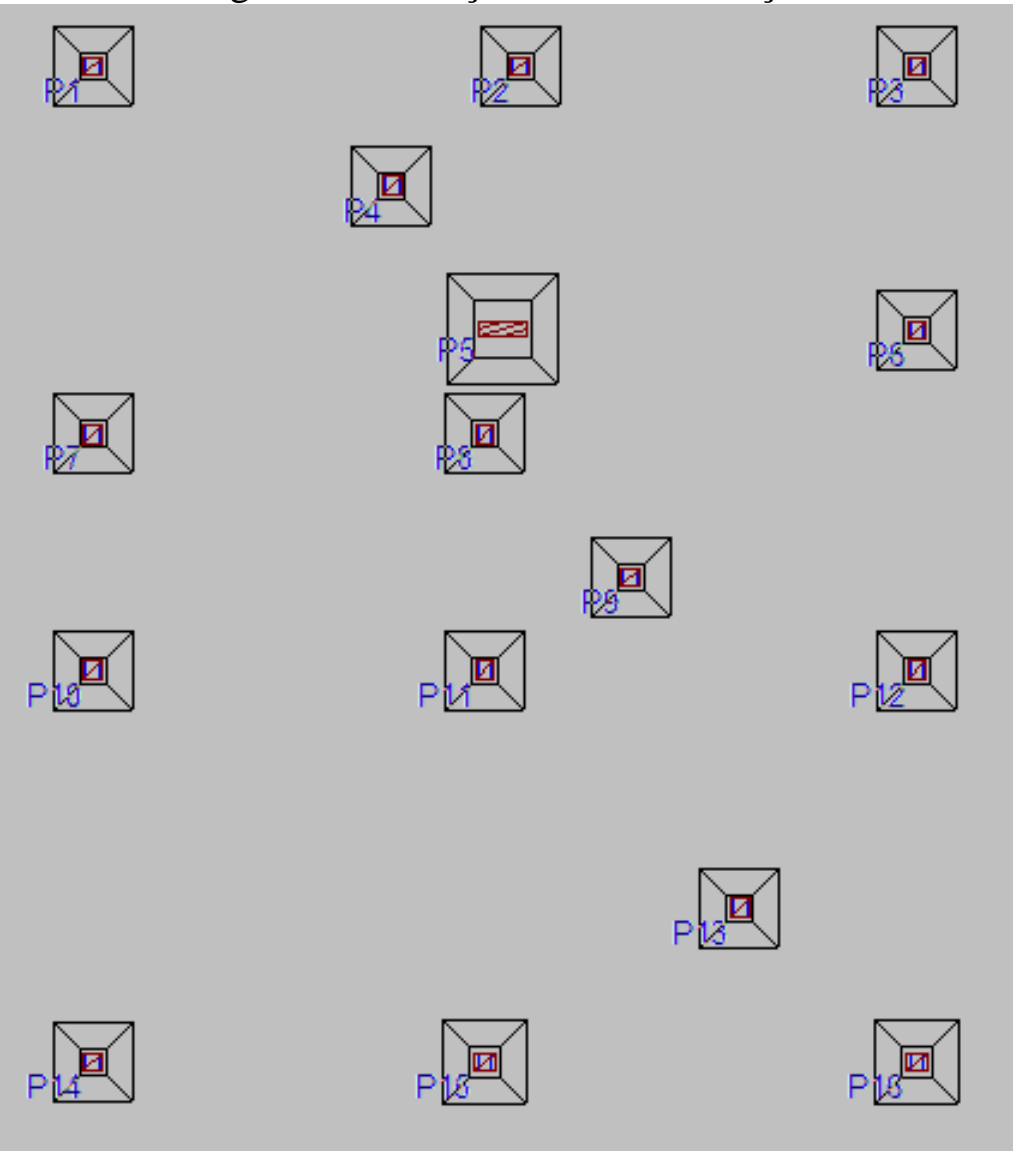

Fonte: Software Cypecad (2017), 2021 


\section{Pontifícia Universidade Catállica $_{\text {a }}$}

\subsubsection{Cálculo da obra}

Após feito todo o pré-dimensionamento é realizado o cálculo do modelo estrutural, ao fazer este cálculo, no software, é gerado um relatório, caso seja necessário, mostrando onde foram encontrados erros de dimensionamento, como mostra a figura 38.

Figura 38 - Relatório de erros

Erros de cálculo da obra 'TCC-novo-R1' (Versão 2017.m)

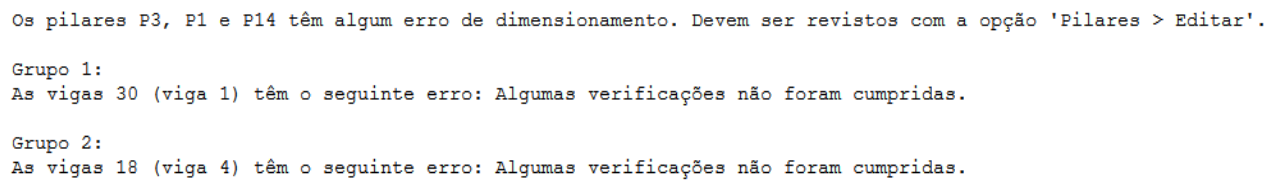

Fonte: Software Cypecad (2017), 2021

Com isso, é possível fazer a correção nos elementos estruturais mencionados neste relatório.

\subsubsection{Correção de erros nos pilares}

Após gerado o relatório de erro no dimensionamento (figura 38), podemos identificar que ele apresentou erro no dimensionamento de alguns pilares, como também mostra a figura 39 (pilares sinalados com um "x" vermelho)

Para corrigir o problema foi feito uma alteração na geometria da seção desses pilares, após esta alteração todos os pilares passaram (pilares sinalados com um"v" verde). Como mostra a figura 40 . 


\section{Pontificia U Universidade C $_{\text {atólica }}$ DO RIO DE JANEIRO}

Figura 39 - Erro nos Pilares

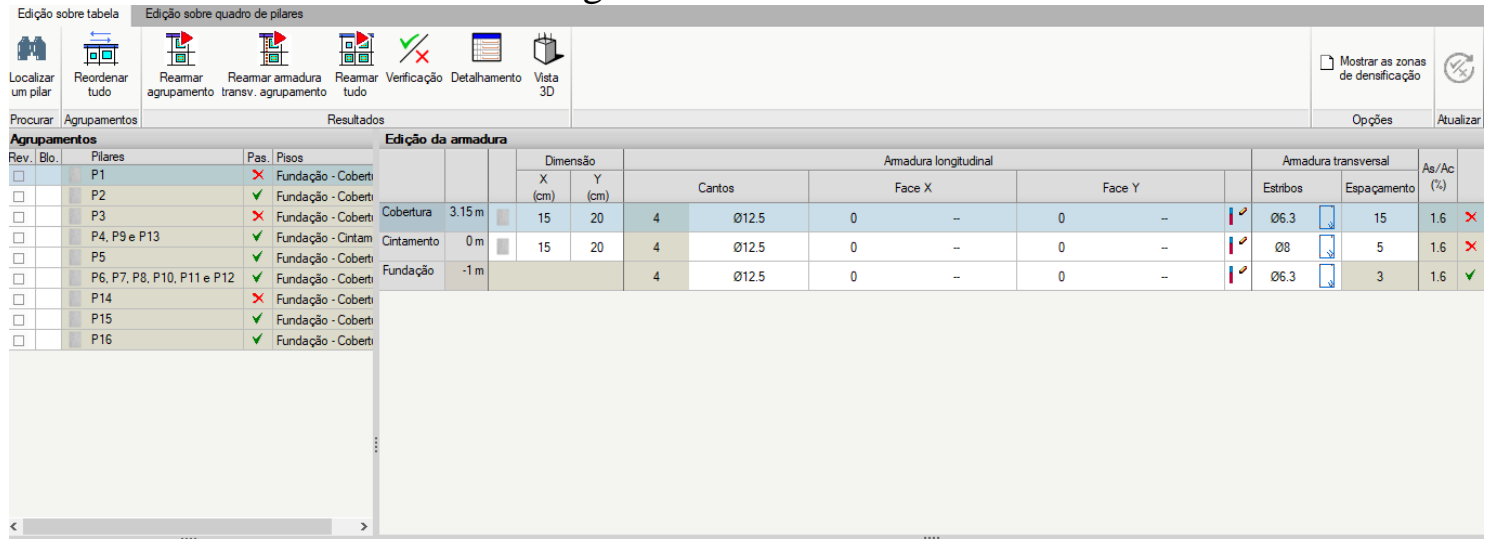

Fonte: Software Cypecad (2017), 2021

Figura 40 - Erro corrigido nos pilares

\begin{tabular}{|c|c|c|c|c|}
\hline \multicolumn{2}{|c|}{ Procurar } & Agrupamentos & \multicolumn{2}{|r|}{ Resultados } \\
\hline \multicolumn{5}{|c|}{ Agrupamentos } \\
\hline Rev. & Blo. & Pilares & Pas. & Pisos \\
\hline$\square$ & & P1 & $\checkmark$ & Fundação - Cobertı \\
\hline$\square$ & & $\mathrm{P} 2$ & $\forall$ & Fundação - Cobertı \\
\hline$\square$ & & P3 & $\checkmark$ & Fundação - Cobertı \\
\hline$\square$ & & P4, P9 e P13 & $\checkmark$ & Fundação - Cintam \\
\hline$\square$ & & P5 & $\checkmark$ & Fundação - Cobertı \\
\hline$\square$ & & P6, P7, P8, P10, P11 e P12 & $\checkmark$ & Fundação - Cobertı \\
\hline$\square$ & & P14 & $\checkmark$ & Fundação - Cobertı \\
\hline$\square$ & & P15 & $\checkmark$ & Fundação - Cobertı \\
\hline$\square$ & & P16 & $\checkmark$ & Fundação - Cobertı \\
\hline
\end{tabular}

Fonte: Software Cypecad (2017), 2021 


\section{Pontifícia Universidade Catálica $_{\text {a }}$

\subsubsection{Correção de erros nas vigas}

O relatório de erro no dimensionamento (figura 38), também apresentou erro em algumas vigas (figuras 41 e 42).

Para corrigir o problema foi feito uma alteração na geometria da seção dessas vigas, a espessura foi alterada de $15 \mathrm{~cm}$ para $20 \mathrm{~cm}$, após esta alteração todas as vigas estão passando (figuras 43 e 44).

Figura 41 - Erro viga 1 grupo 1

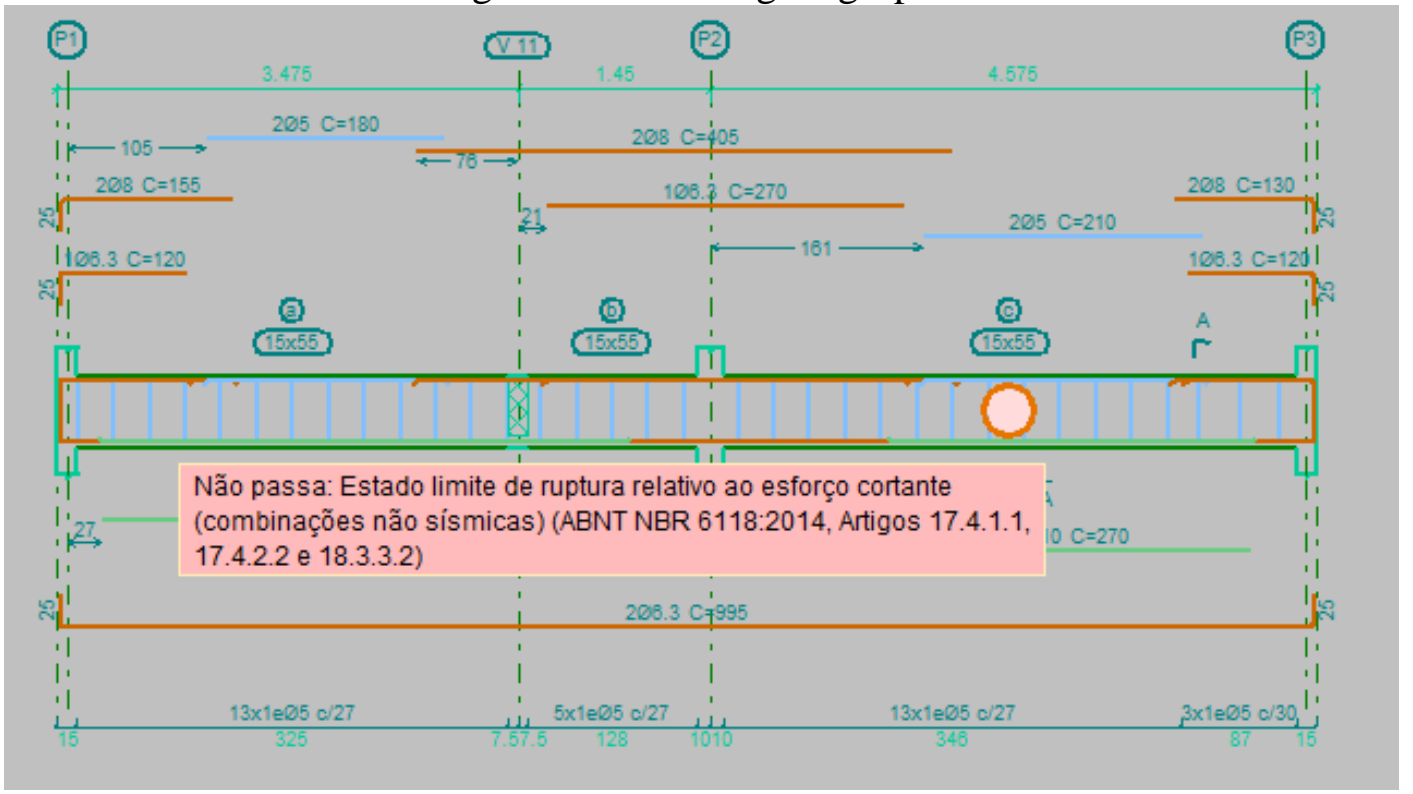

Fonte: Software Cypecad (2017), 2021 


\section{Pontifícia Universidade Catálica

Figura 42 - Erro viga 4 grupo 2

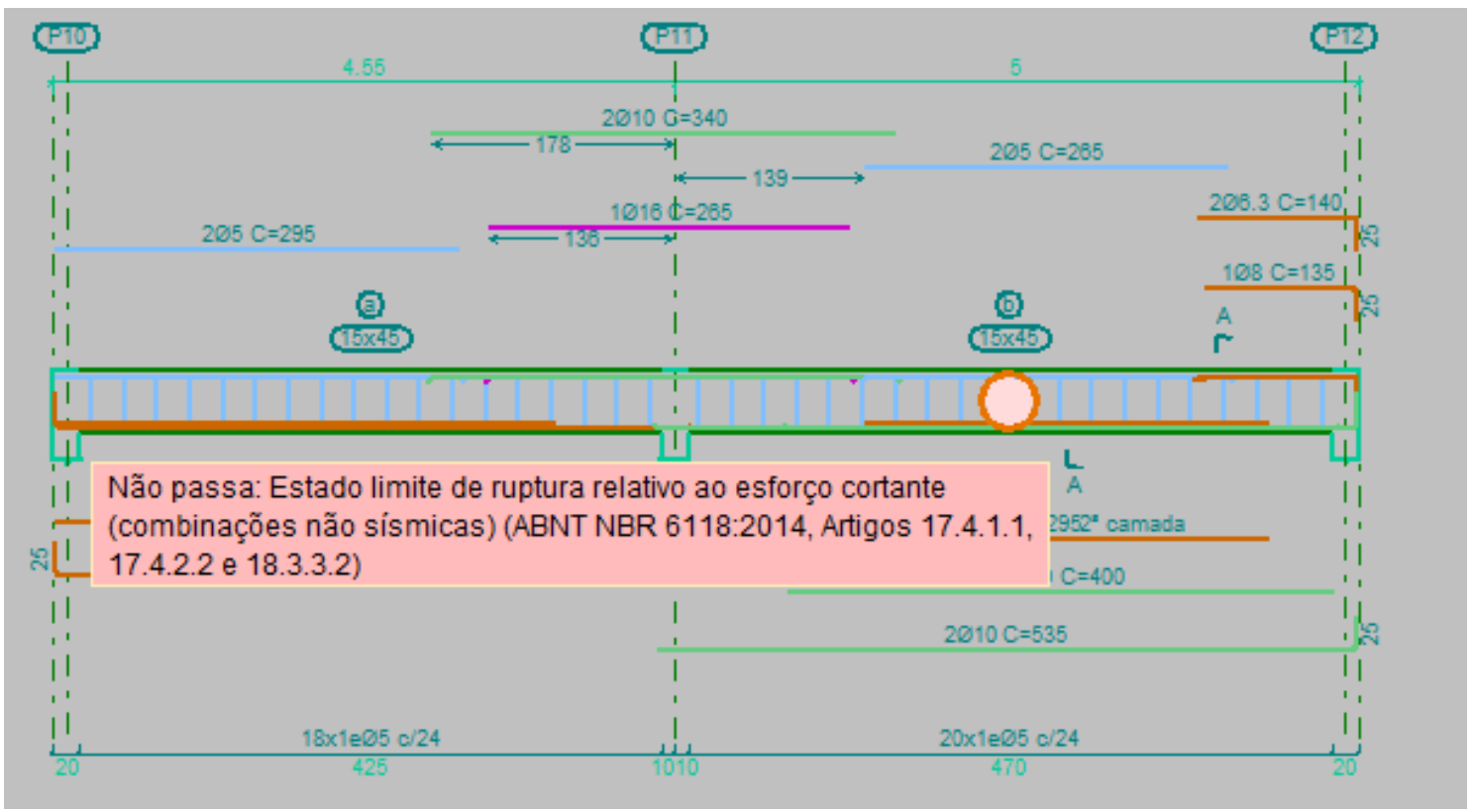

Fonte: Software Cypecad (2017), 2021

Figura 43 - Erro corrigido da viga 1 grupo 1

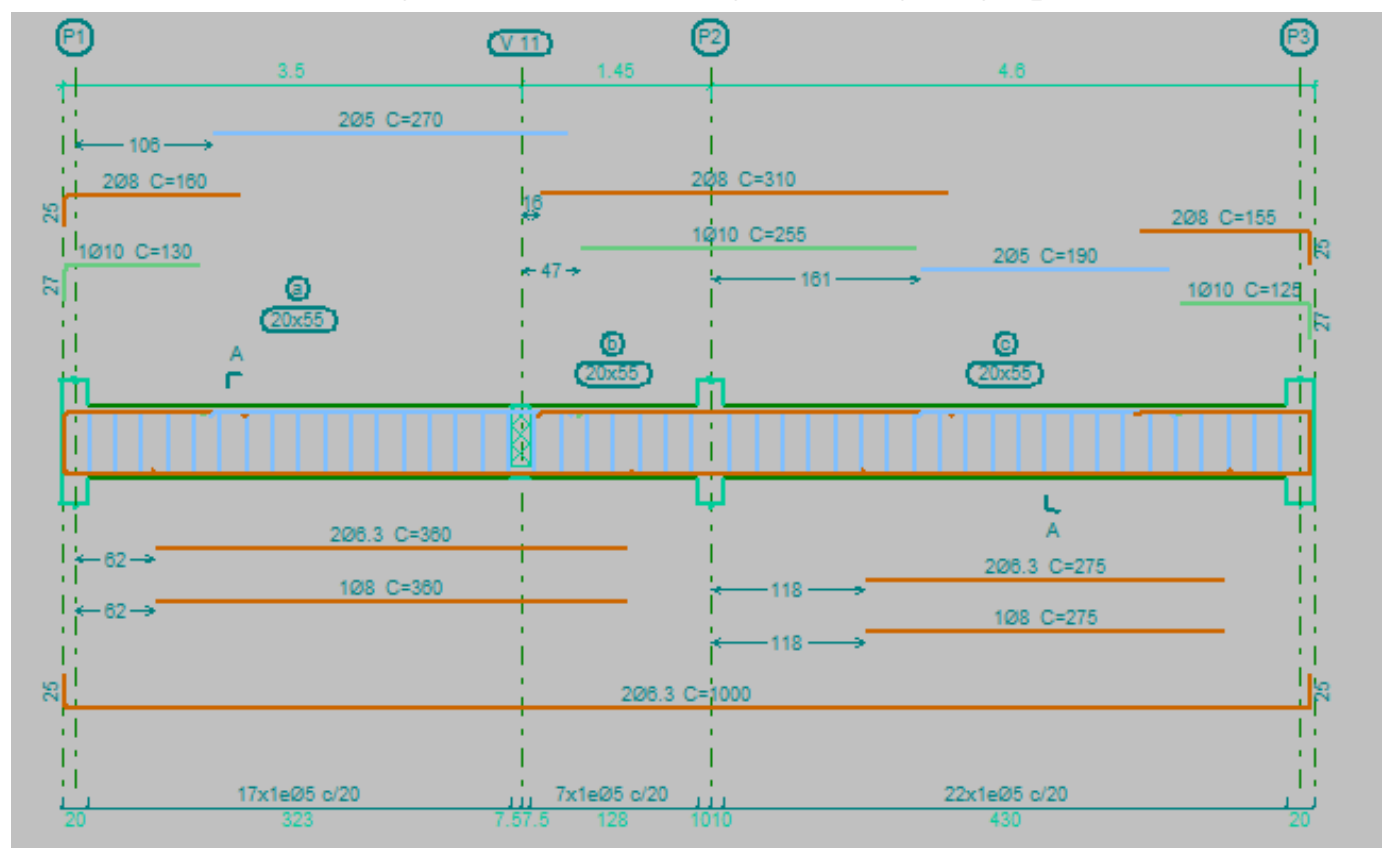

Fonte: Software Cypecad (2017), 2021 


\section{Pontifícia Universidade Catállica $_{\text {a }}$

Figura 44 - Erro corrigido viga 4 grupo 2

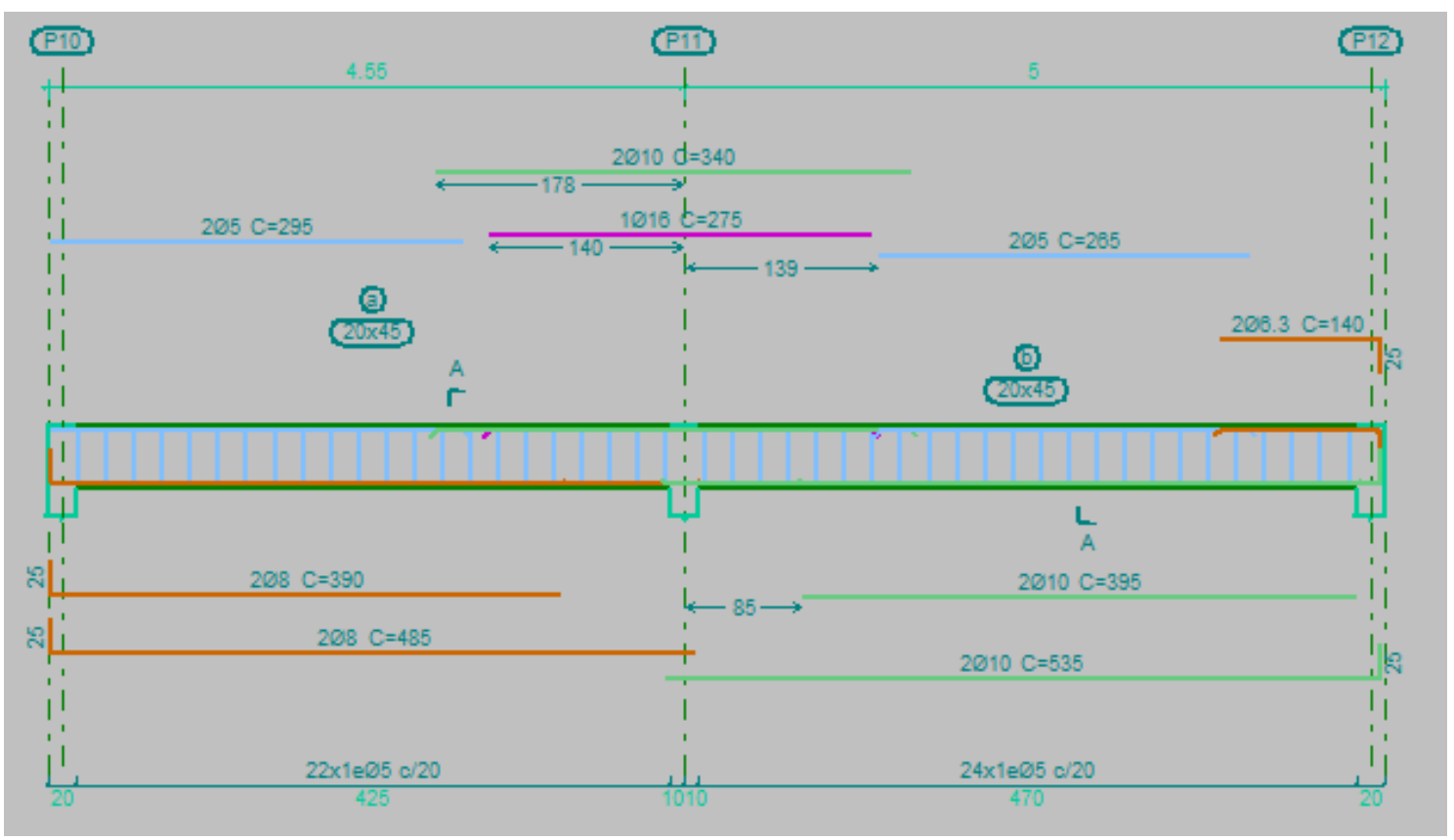

Fonte: Software Cypecad (2017), 2021

Mesmo com essa alteração na seção da viga, não houve necessidade de alterar a carga das alvenarias, como foi mencionado no item 3.4.8, pois as alturas das vigas não foram alteradas. 


\section{Pontifícia Universidade Cháólica

\subsubsection{Modelo estrutural 3D}

Após feito todos os cálculos, podemos observar na figura 45 o modelo estrutural

3D da edificação.

Figura 45 - Modelo estrutural 3D

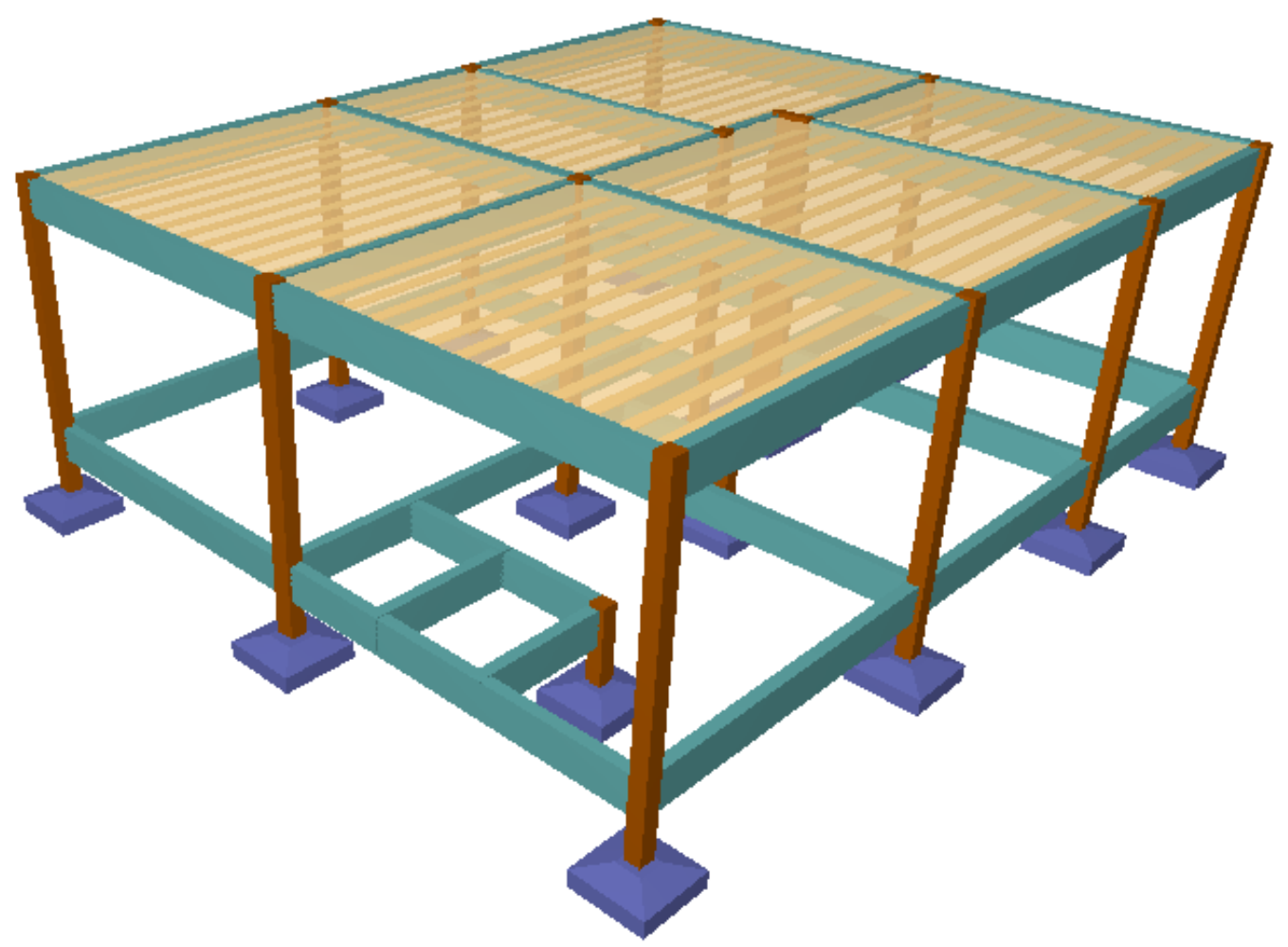

Fonte: Software Cypecad (2017), 2021

\subsubsection{Otimização da armadura das vigas}

Por se tratar de uma obra "manual" foi feito uma otimização da armadura das vigas para uma maior segurança e maior agilidade no processo construtivo. 


\section{Pontifícia Universidade Catálica

Figura 46 - Viga 1 não otimizada

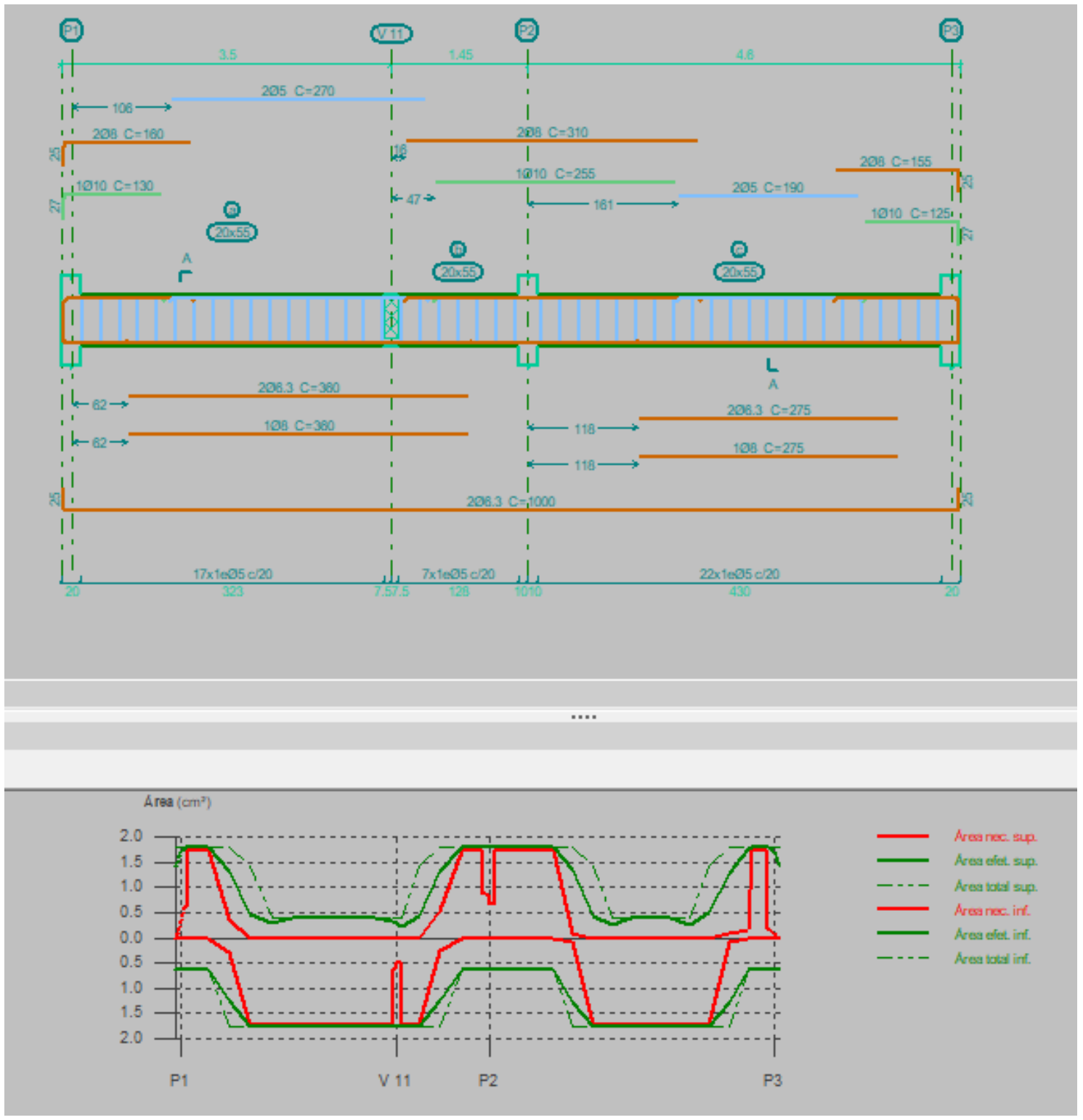

Fonte: Software Cypecad (2017), 2021

Na parte superior da figura 46, podemos observar uma grande quantidade de ferros com diâmetros diferentes e em várias posições também diferentes, isso dificulta o processo construtivo na obra o tornando mais lento.

Na parte inferior da figura 46 é representado o gráfico da área de aço necessária (linha vermelha) e a área de aço efetiva (linha verde). Para a viga poder passar a linha verde precisa estar acima da linha vermelha (no caso da armadura superior) e abaixo da 


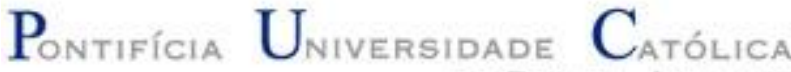

linha vermelha (no caso da armadura inferior). Podemos observar que em alguns pontos a linha verde está muito próxima da linha vermelha o que acaba não deixando muita margem para erro, este fato compromete a segurança do projeto.

Para corrigir essas imperfeições foi feito uma alteração na armadura da viga, como pode ser visto na figura 47.

Figura 47 - Viga 1 otimizada

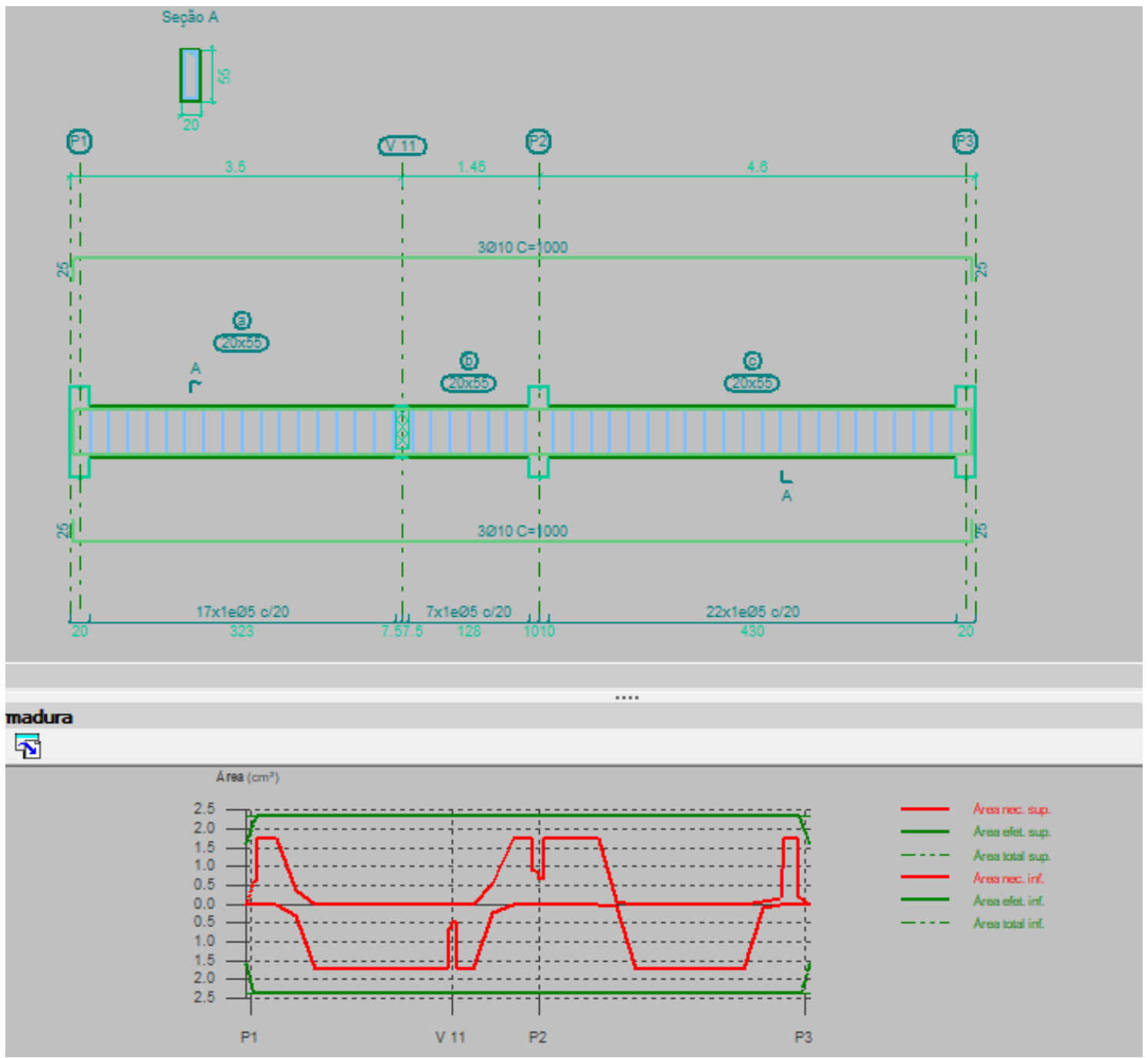

Fonte: Software Cypecad (2017), 2021

Após a alteração na armadura da viga podemos observar a presença de apenas 1 tipo de barra e que também as linhas de área de aço estão mais afastadas, isso torna o processo construtivo mais rápido e mais seguro. 


\section{Pontifícia Universidade Catálica $_{\text {a }}$

Este processo foi feito em todas as vigas, ao final deste trabalho será apresentado os desenhos de forma e armadura deste projeto com todas as armaduras de viga já otimizadas.

Esta otimização não altera o modelo estrutural 3D (item 3.4.13)

\section{INTEGRAÇÃO ENTRE OS PROJETOS}

\subsection{Exportação do ifc do Cypecad para o Revit}

Após a realização do projeto estrutural foi feito a sua exportação, com ifc, para o Revit, de modo a fazer a compatibilização entre as disciplinas e corrigir as interferências. 


\section{Pontifícia Universidade Católica

Figura 48 - Exportação do ifc

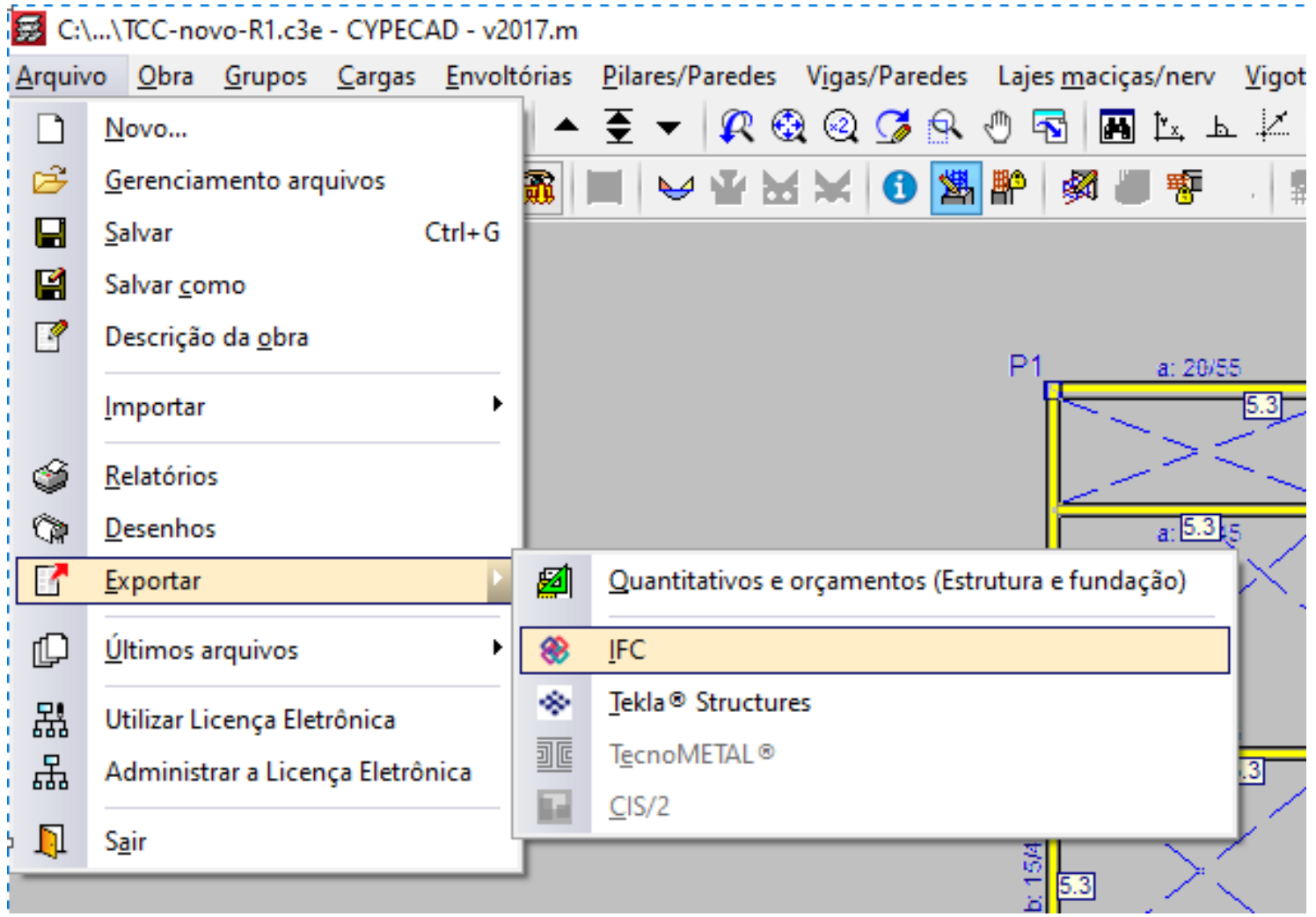

Fonte: Software Cypecad (2017), 2021

Figura 49 - Modelo estrutural exportado para o Revit, com ifc

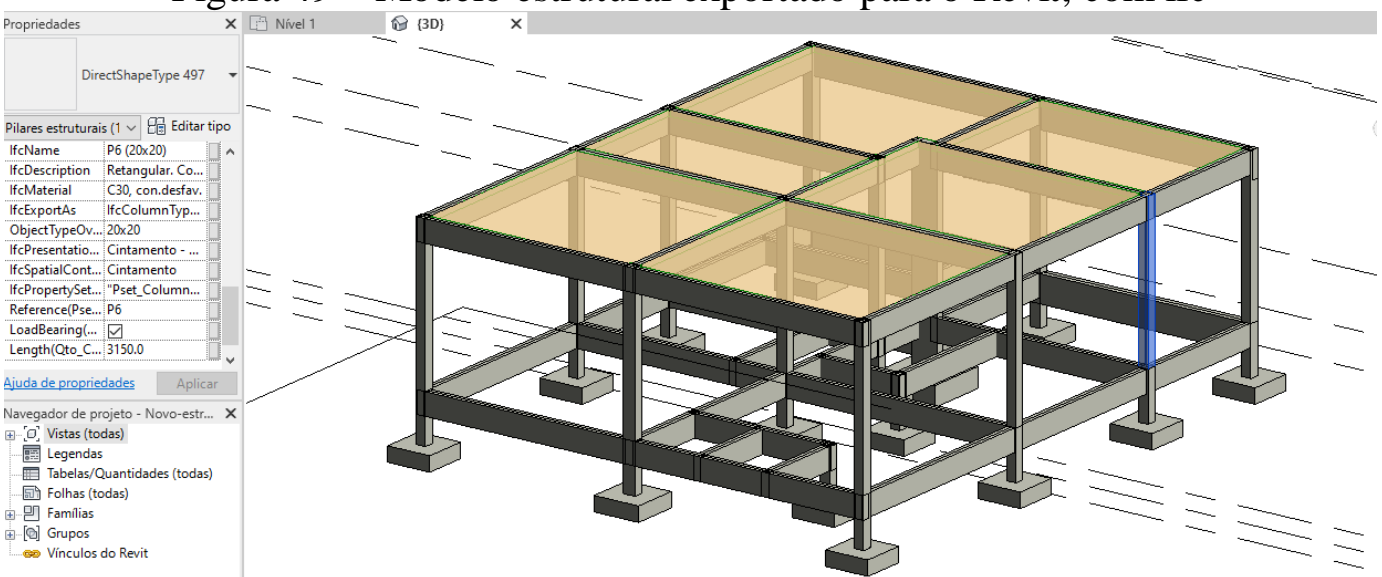

Fonte: Software Revit (2021), 2021 


\section{Pontifícia Universidade Catálica

Ao exportar o modelo estrutural para o Revit, com o ifc (figura 48), podemos ver

que além do desenho ele também mostra várias informações sobre o elemento estrutural selecionado, como pode ser visto na tabela a esquerda da figura 49.

\subsection{Sobreposição dos projetos}

Tendo, tanto o projeto estrutural quanto o projeto arquitetônico no Revit, são feitos a sobreposição (figuras 50 e 51) entre eles para fazer a checagem das interferências e as correções.

Figura 50 - Sobreposição dos projetos 2D

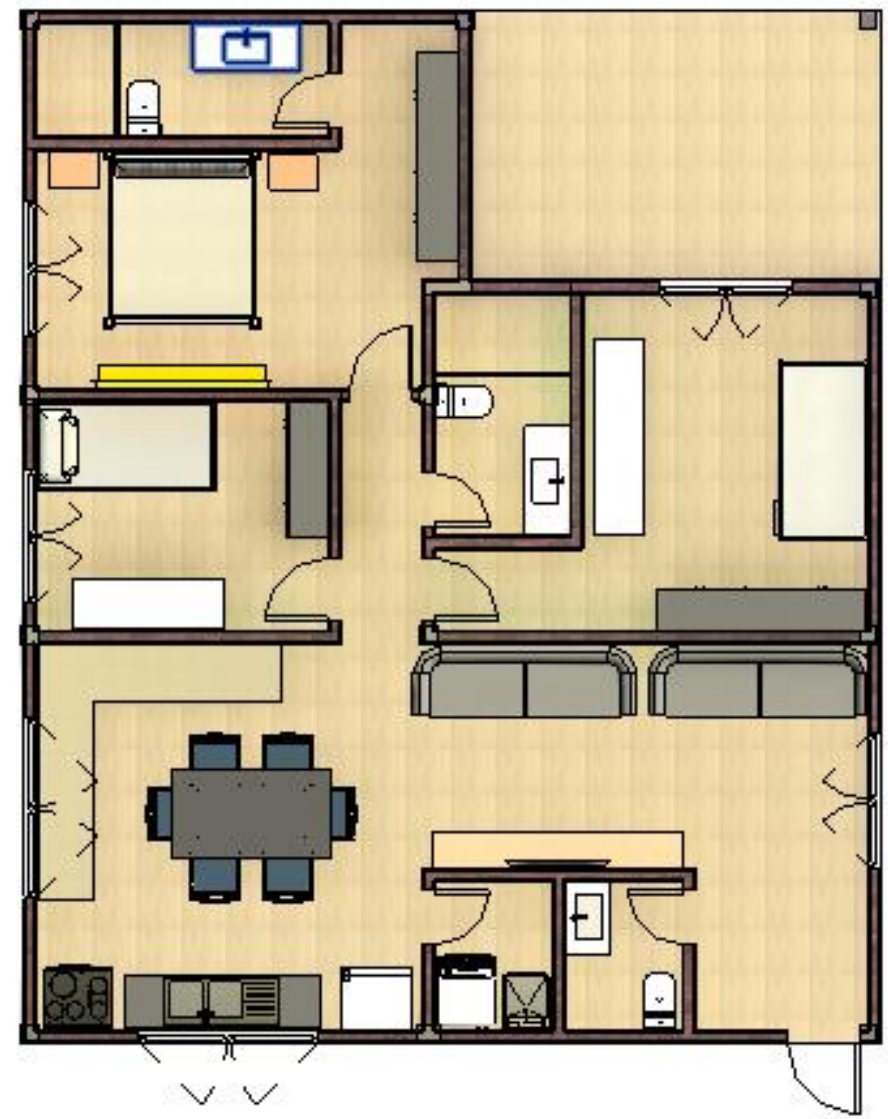

Fonte: Software Revit (2021), 2021 


\section{Pontifícia Universidade Catálica

Figura 51 - Sobreposição dos projetos 3D

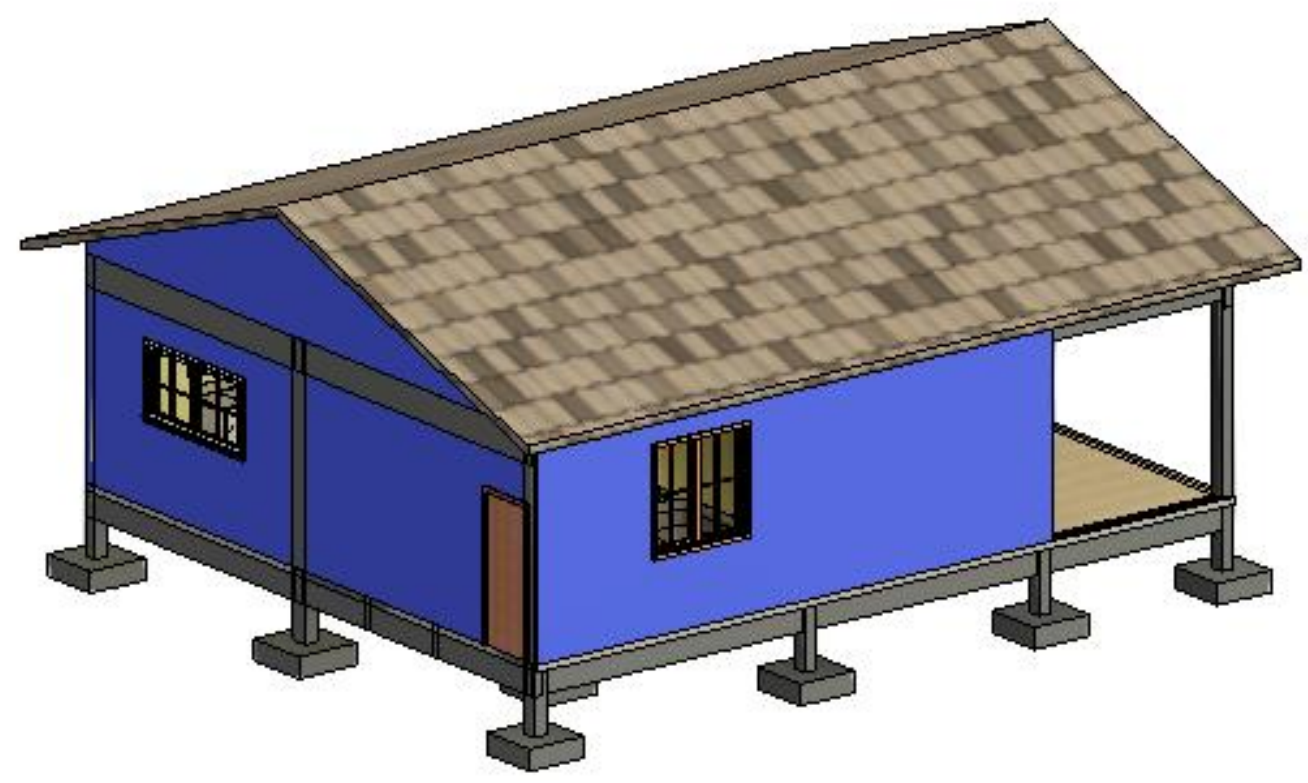

Fonte: Software Revit (2021), 2021

Ao comparar as figuras 13 e 15 com as figuras 50 e 51, podemos perceber nestas últimas a presença dos elementos estruturais.

\subsection{Checagem das interferências}

Ao fazer a sobreposição podemos verificar 2 principais interferências: pilar dentro da parede (figura 52) e viga dentro da parede (figura 53). 


\section{Pontifícia Universidade Católlca DO RIO DE JANEIRO}

Figura 52 - Pilar dentro da parede

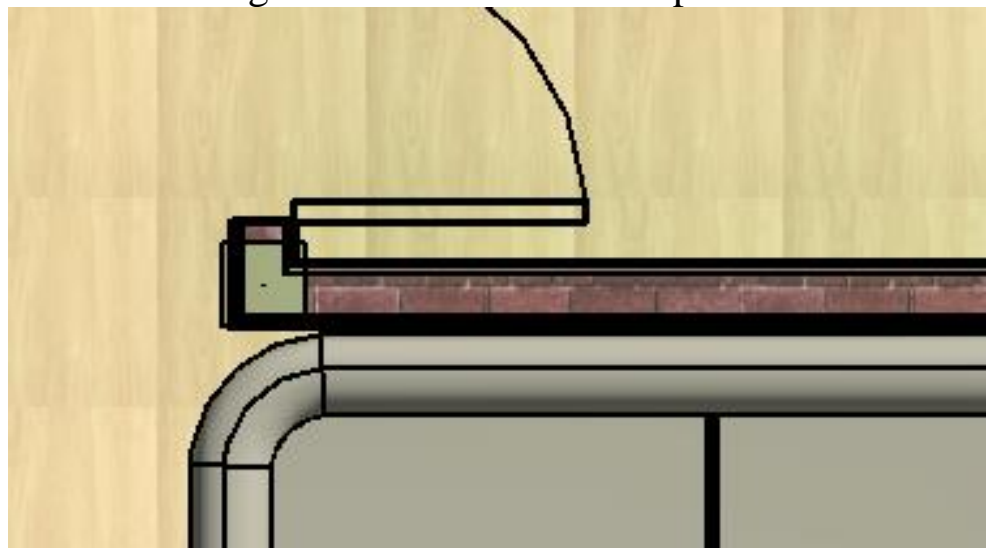

Fonte: Software Revit (2021), 2021

Figura 53 - Viga dentro da parede

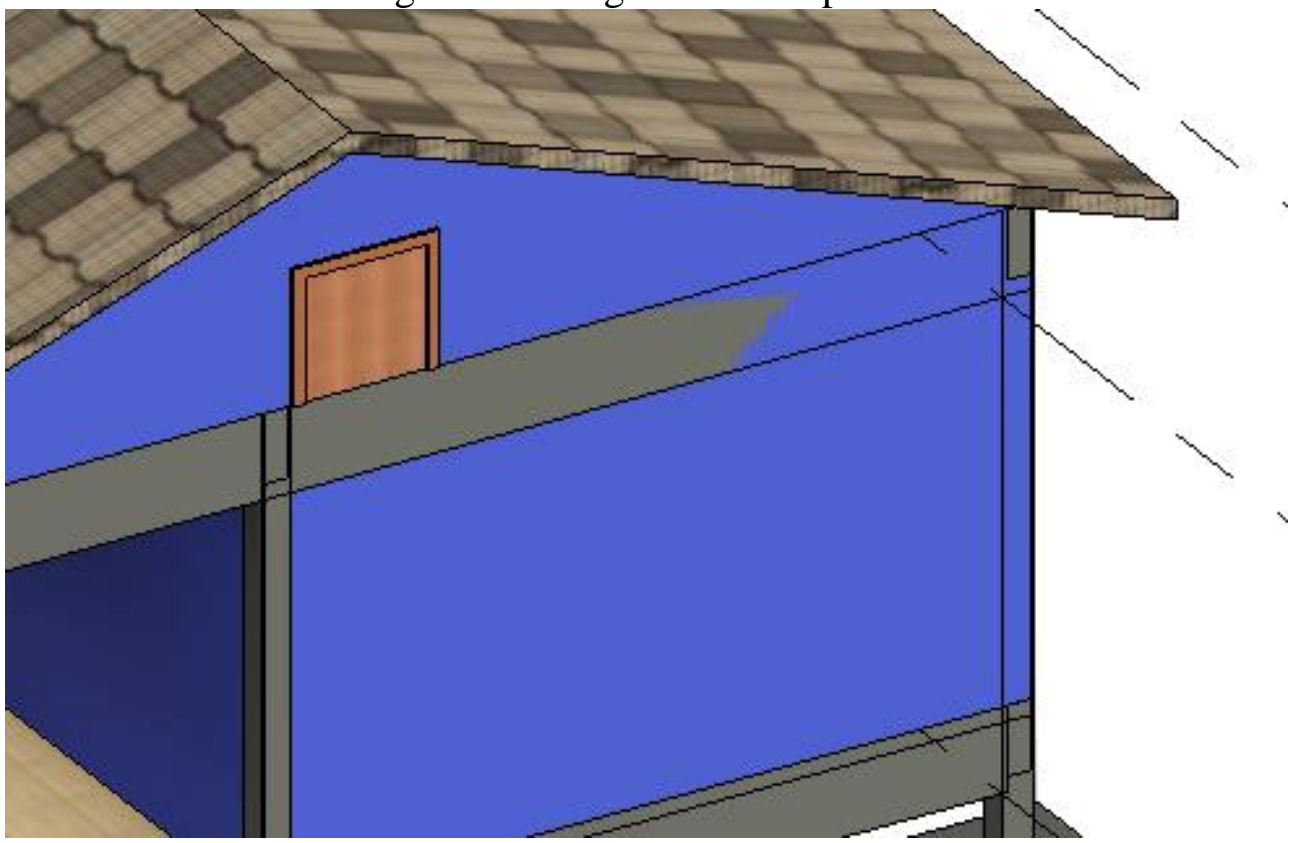

Fonte: Software Revit (2021), 2021 


\section{Pontifícia Universidade Catállica $_{\text {a }}$}

\subsection{Correção das interferências}

Ao verificar as interferências foram feitas as devidas correções, na figura 54 podemos ver a correção dos pilares dentro das paredes e na figura 55 podemos ver a correção das vigas dentro das paredes

Figura 54 - Interferência pilar/parede corrigida

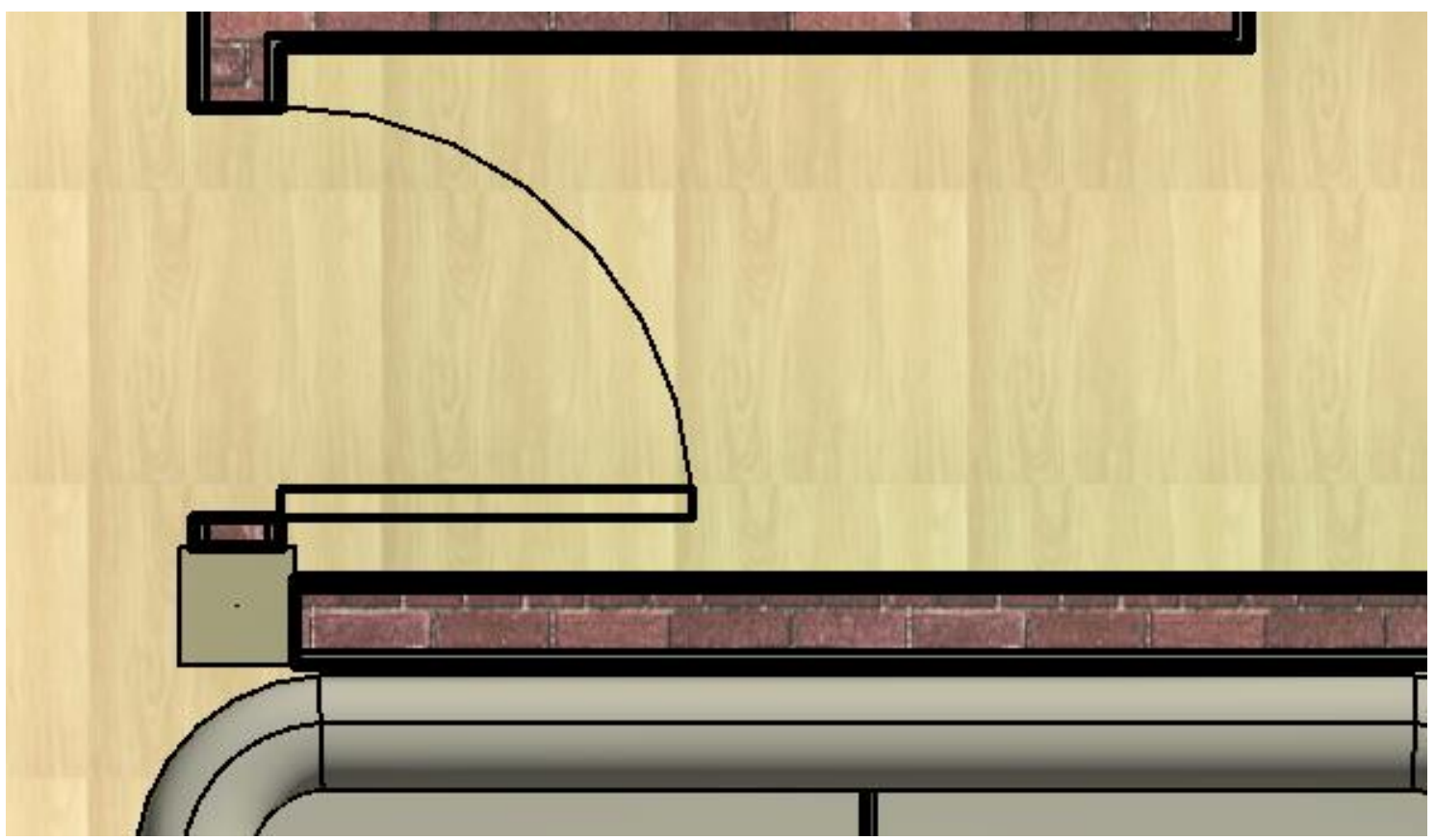

Fonte: Software Revit (2021), 2021 


\section{Pontifícia Universidade Cátólica \\ DO RIO DE JANEIRO}

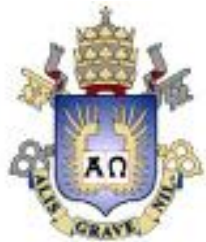

Figura 55 - Interferência viga/parede corrigida

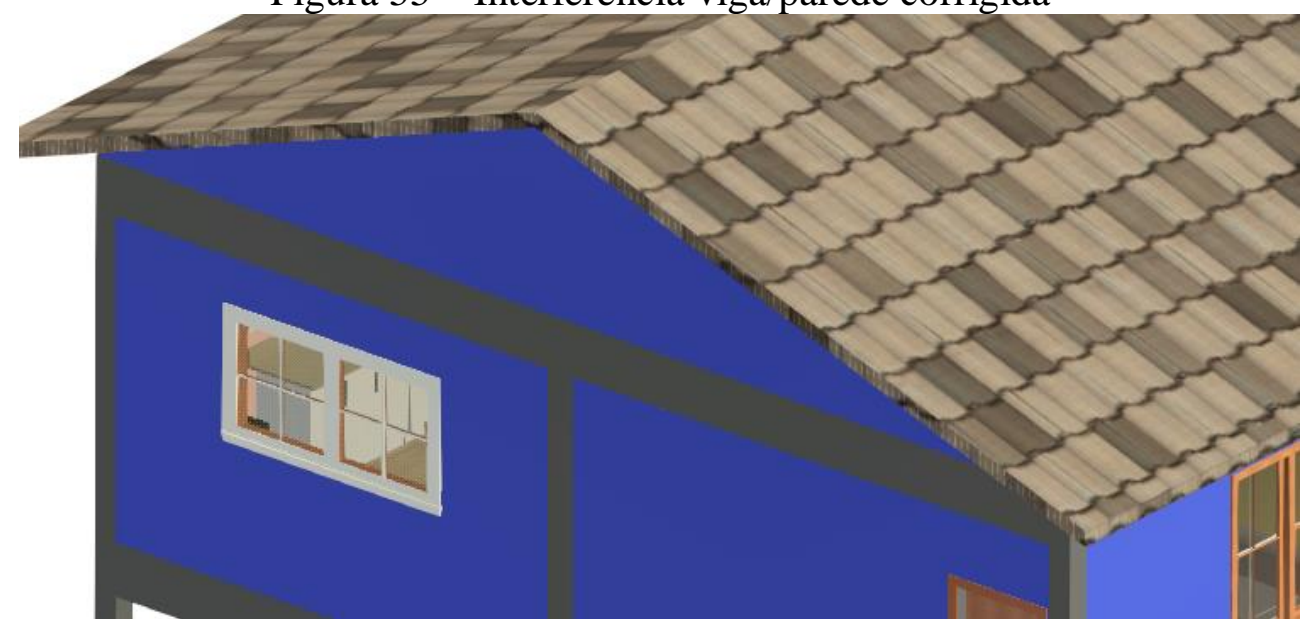

Fonte: Software Revit (2021), 2021

\subsection{Integração entre os projetos finalizados}

Nas imagens abaixo podemos ver as plantas, cortes e o modelo 3D do projeto finalizado. 


\section{Pontifícia Universidade Católlca

Figura 56 - Planta nível térreo

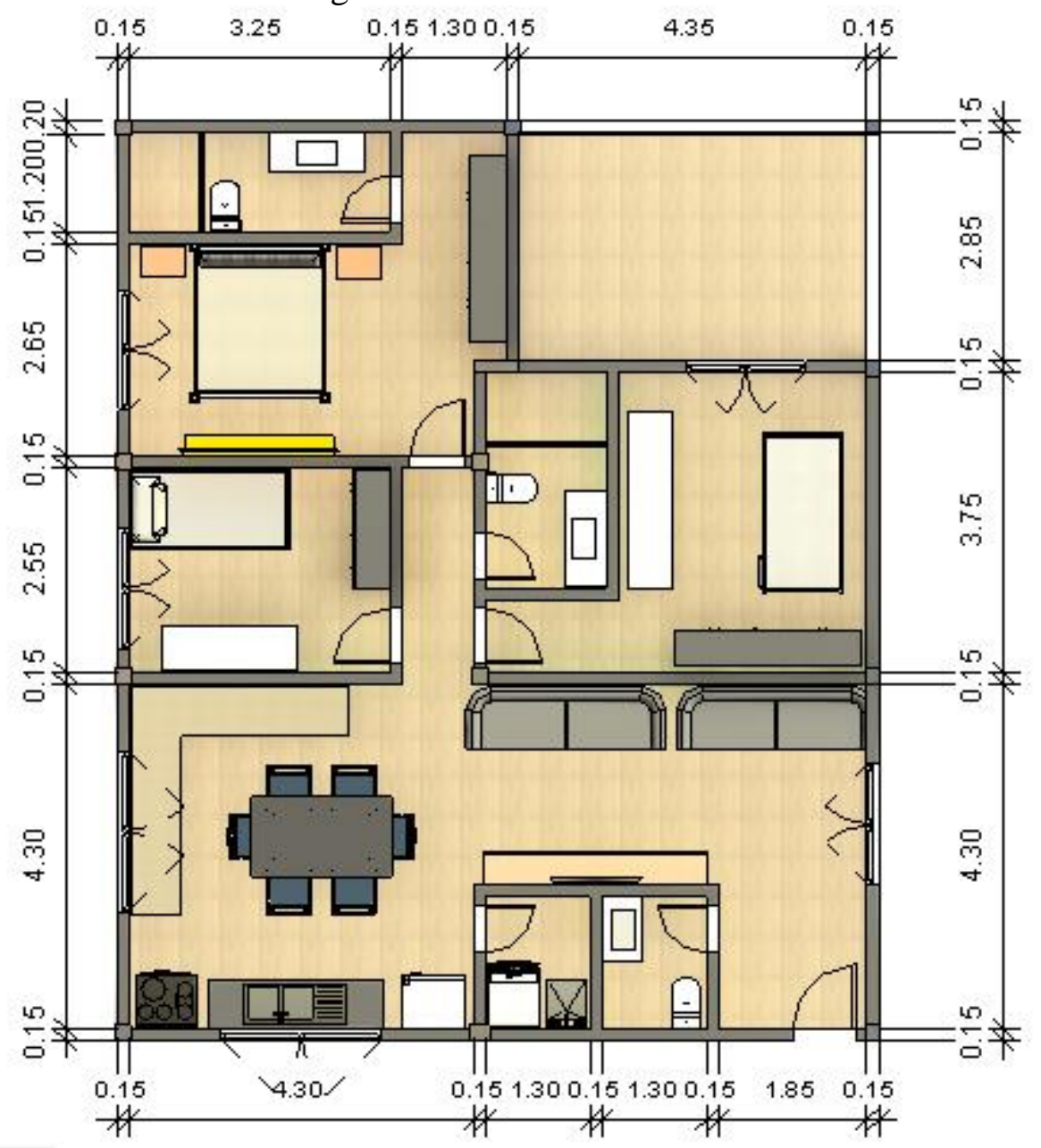

Fonte: Software Revit (2021), 2021 


\section{Pontifícia Universidade Catálica $_{\text {ate }}$ DO RIO DE JANEIRO}

Figura 57 - Corte 1

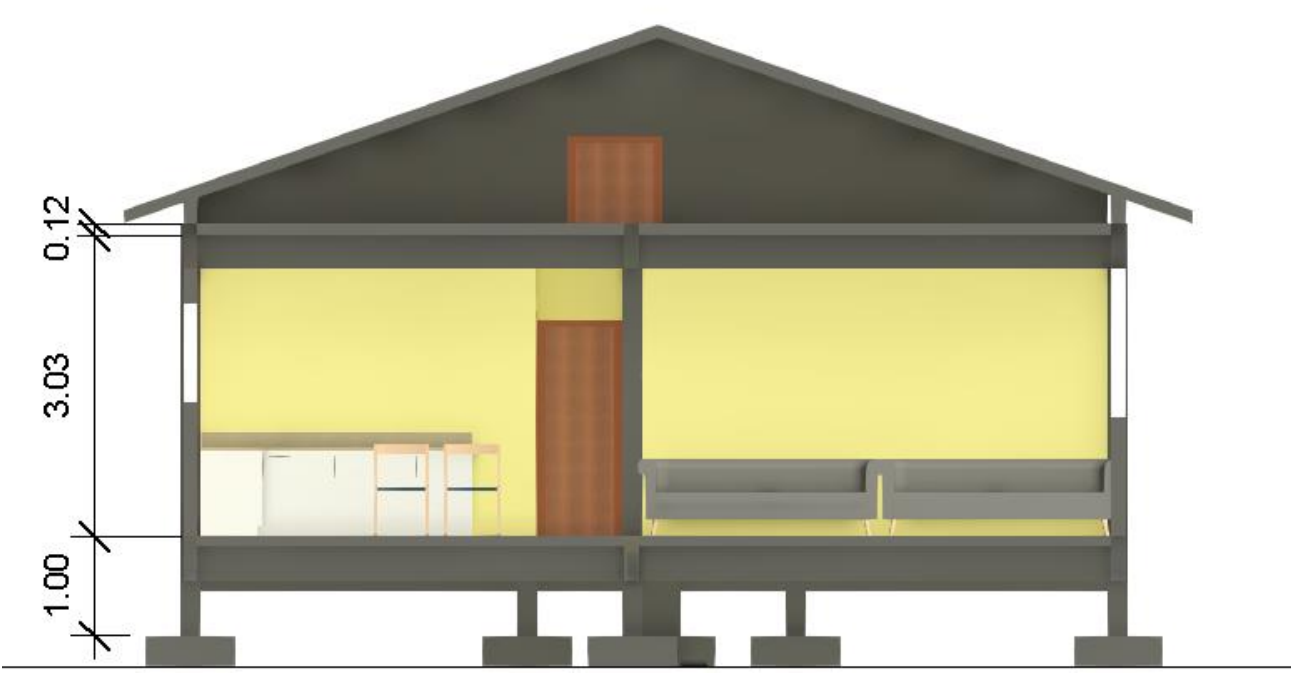

Fonte: Software Revit (2021), 2021

Figura 58 - Corte 2

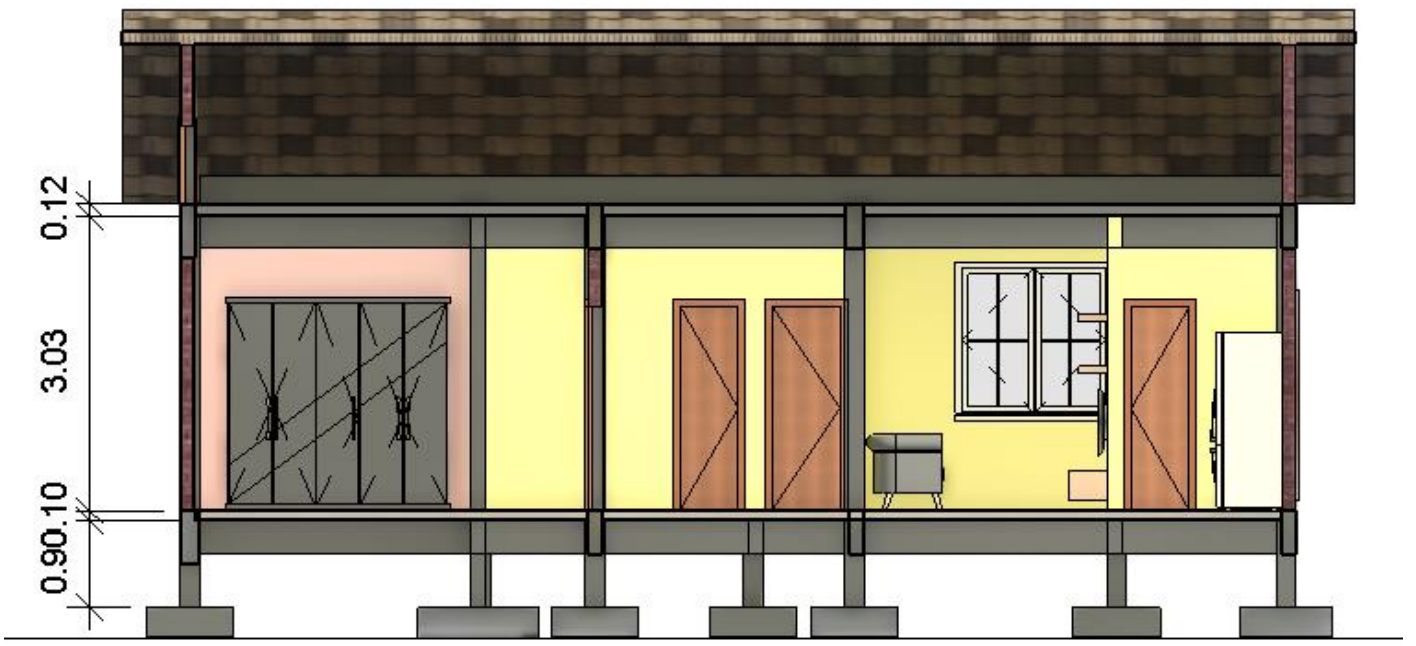

Fonte: Software Revit (2021), 2021 


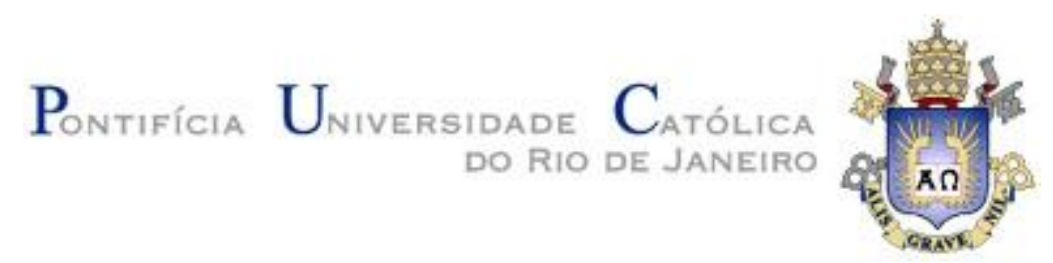

Figura 59 - Modelo 3D

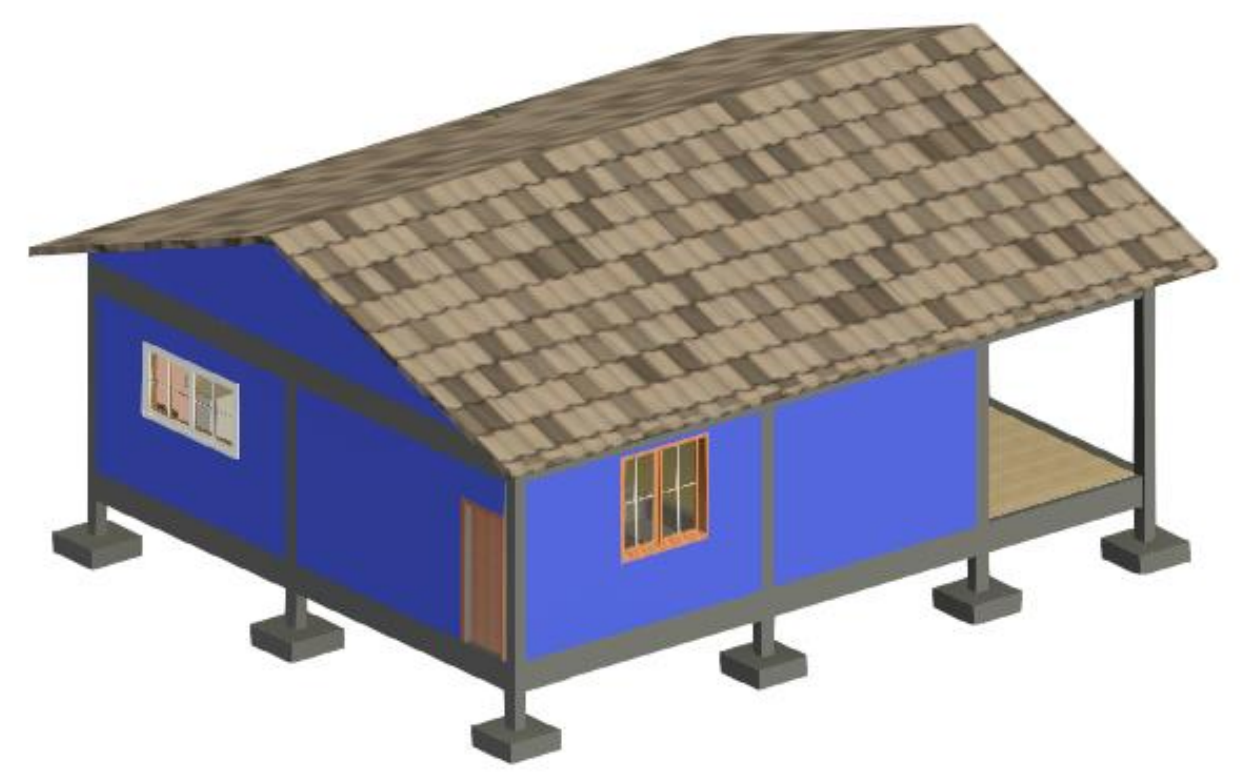

Fonte: Software Revit (2021), 2021 


\section{Pontifícia Universidade Catálica $_{\text {a }}$

\section{DESENHOS DO PROJETO ESTRUTURAL}

Abaixo seguem os desenhos (figuras 60 a 85) de forma e armadura do projeto estrutural, que foram exportados do Cypecad para o AutoCAD

Figura 60 - Forma cintameno

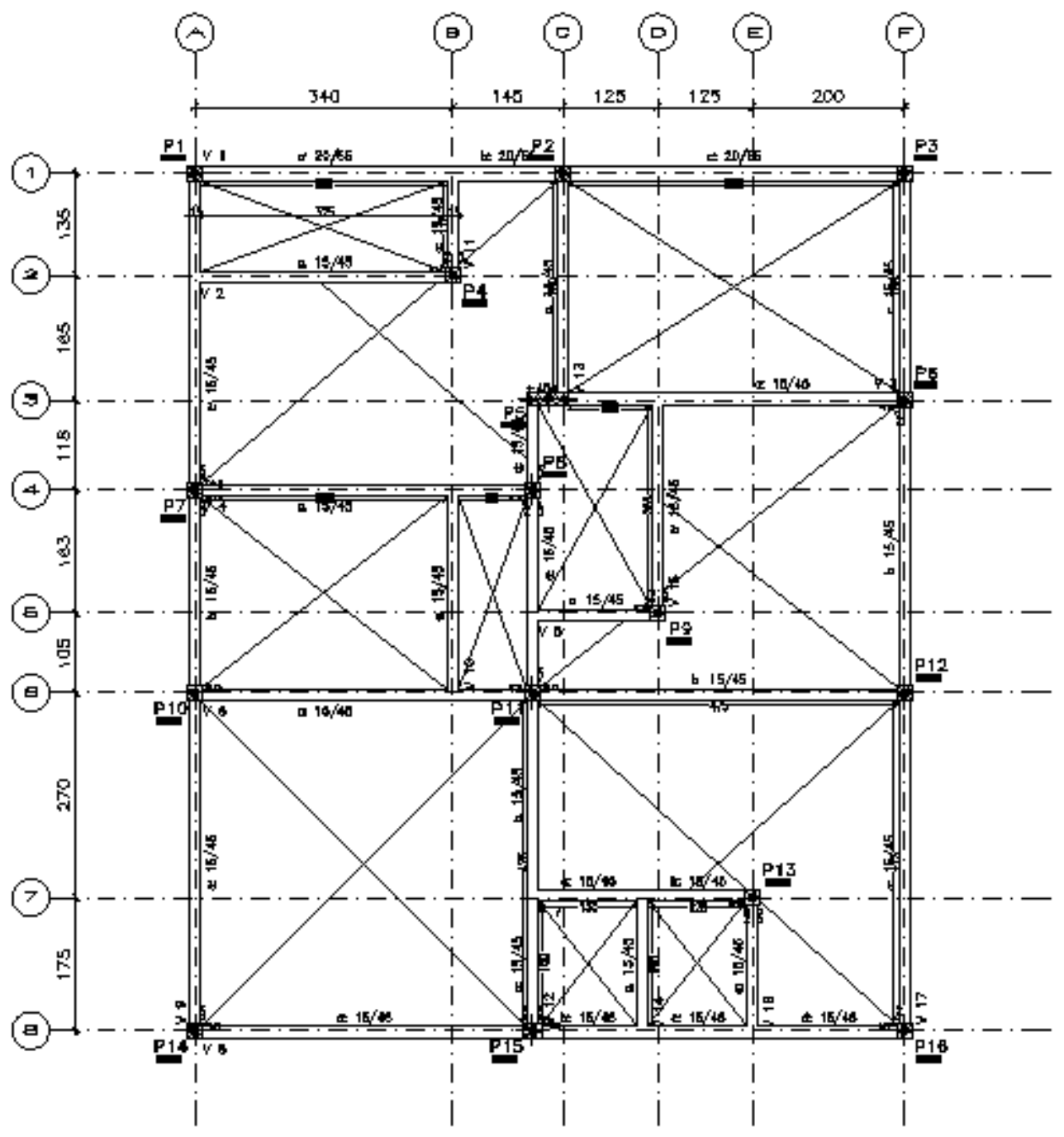

Fonte: Software AutoCAD (2019), 2021 


\section{Pontifícia Universidade Católlca

Figura 61 - forma cobertura

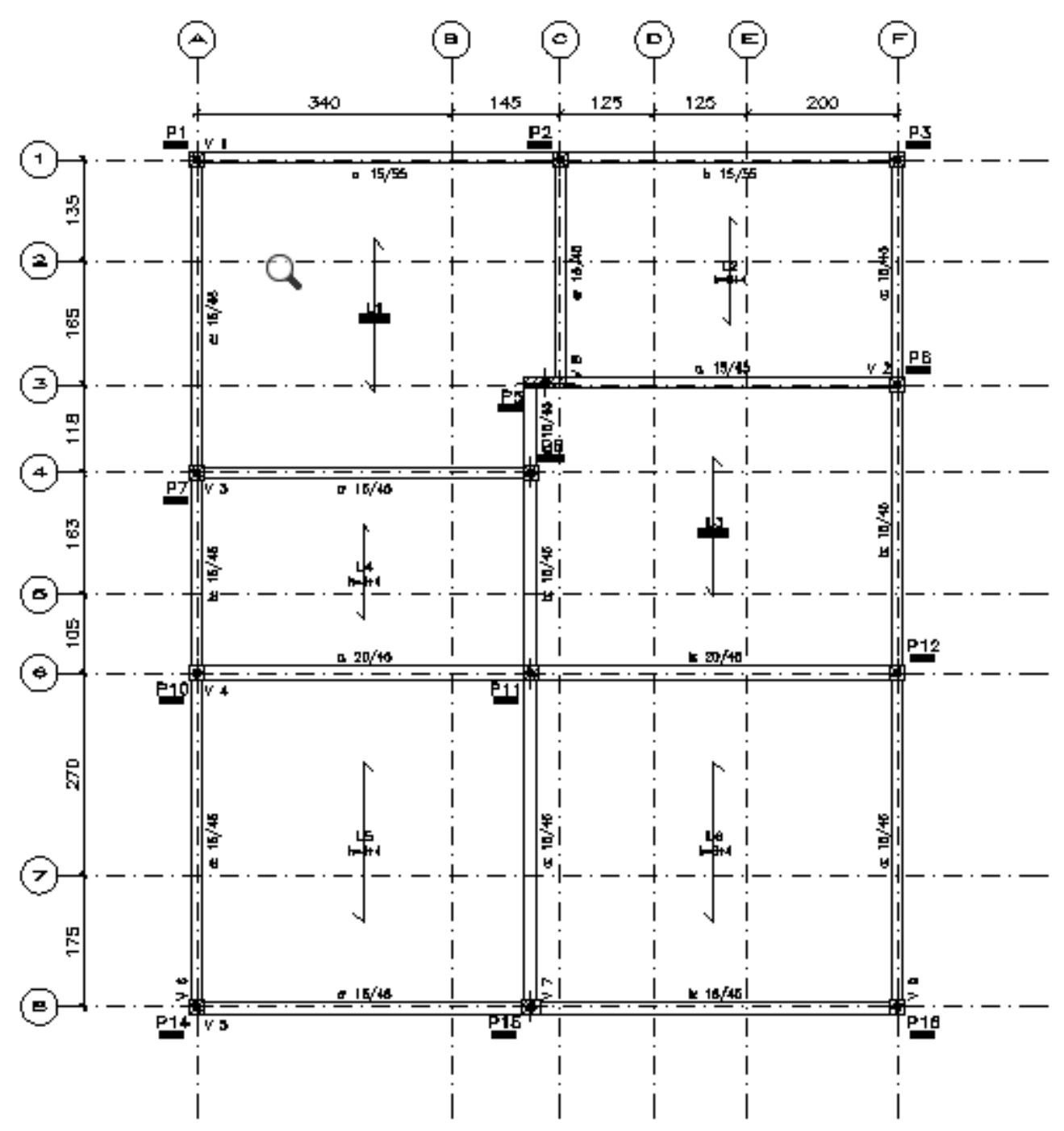

Fonte: Software AutoCAD (2019), 2021 


\section{Pontifícia Universidade Catálica $_{\text {a }}$ DO RIO DE JANEIRO}

Figura 62 - Armadura Pilar P1

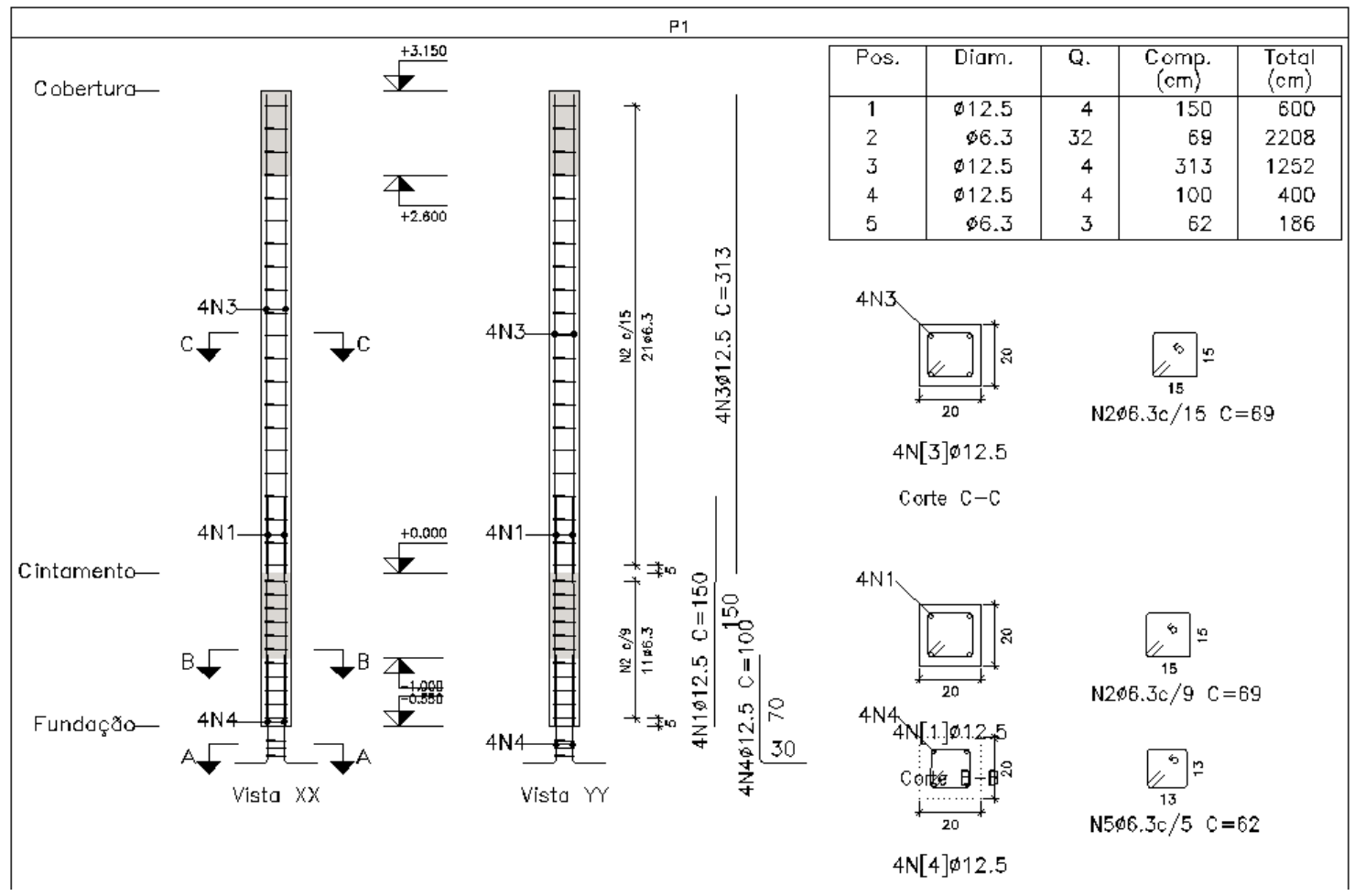

Fonte: Software AutoCAD (2019), 2021 


\section{Pontifícia Universidade Catálica $_{\text {a }}$ DO RIO DE JANEIRO}

Figura 63 - Armadura Pilares P2 e P3

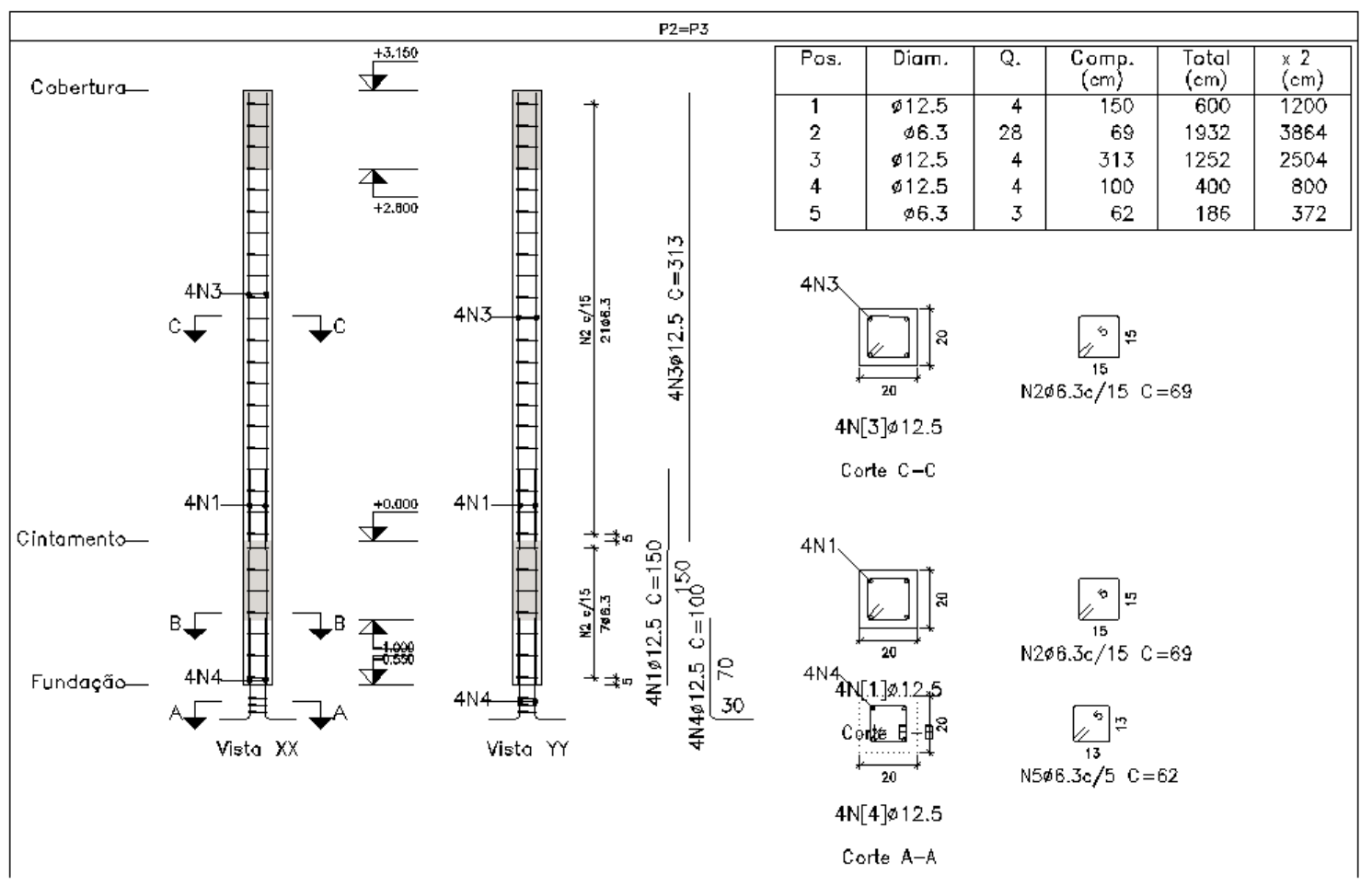

Fonte: Software AutoCAD (2019), 2021 


\section{Pontifícia Universidade Católlca DO RIO DE JANEIRO}

Figura 64 - Armadura Pilares P4, P9 e P13

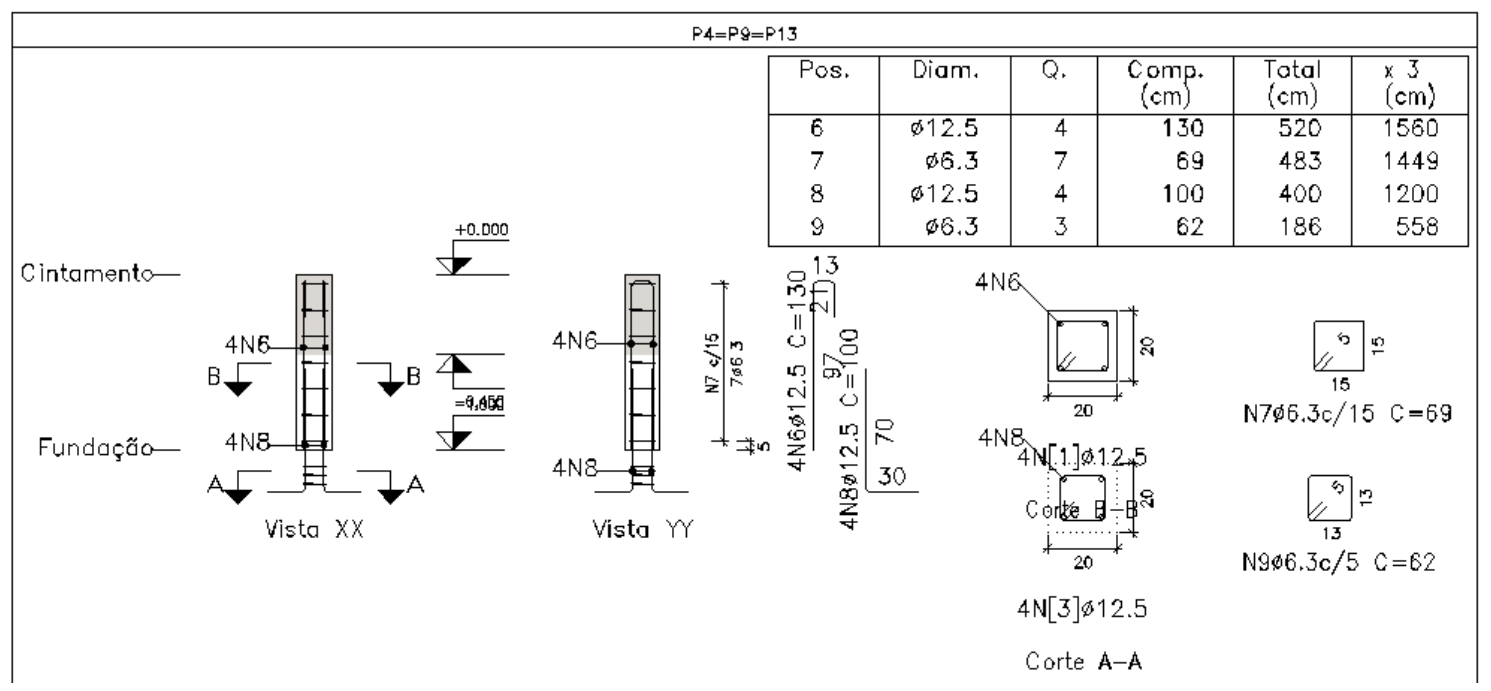

Fonte: Software AutoCAD (2019), 2021

Figura 65 - Armadura Pilar P5

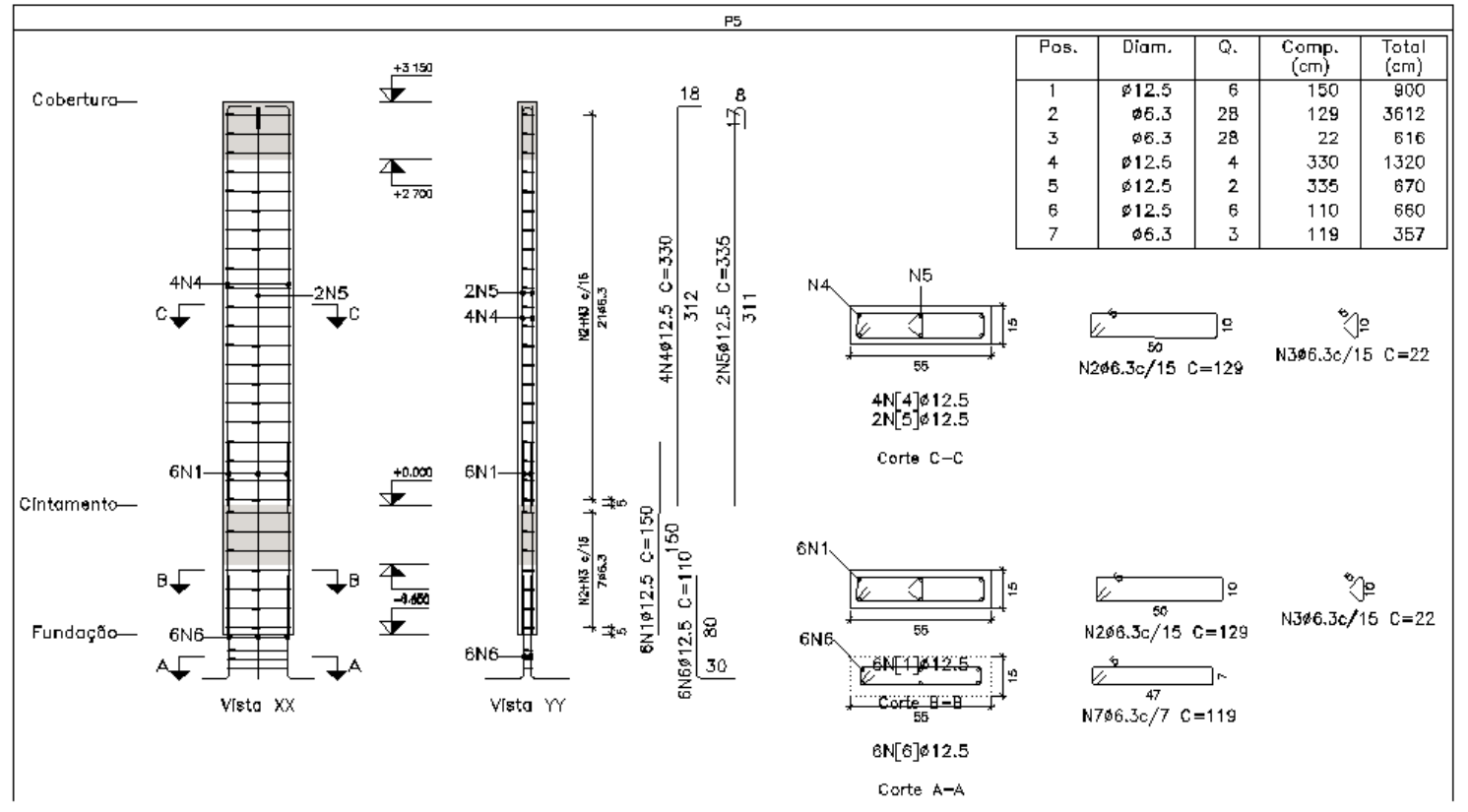

Fonte: Software AutoCAD (2019), 2021 


\section{Pontifícia Universidade Catálica DO RIO DE JANEIRO}

Figura 66 - Armadura Pilares P6, P7, P8, P10, P11, P12 e P14

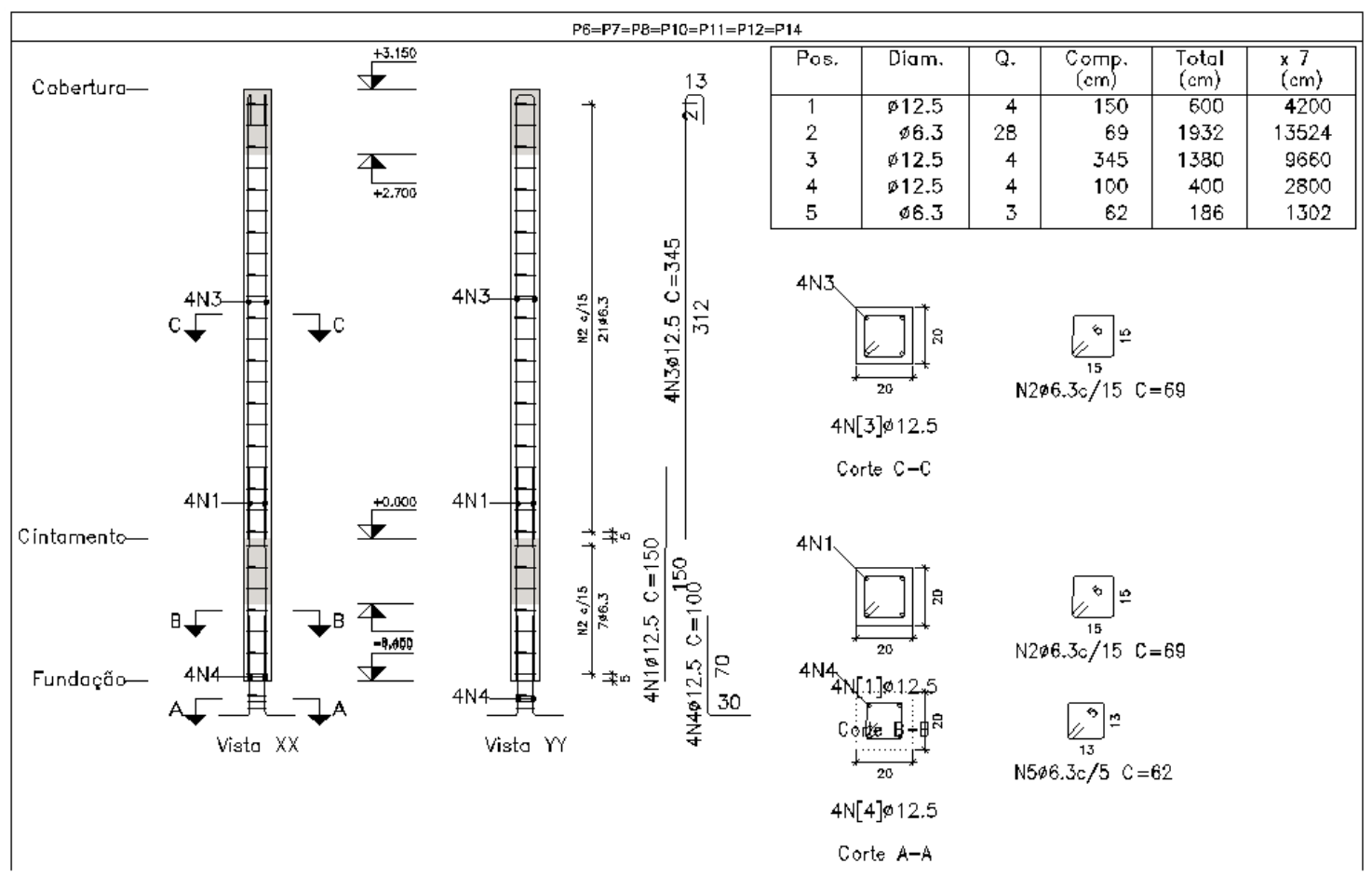

Fonte: Software AutoCAD (2019), 2021 


\section{Pontifícia Universidade Católlica $_{\text {a }}$

Figura 67 - Armadura Pilar 15

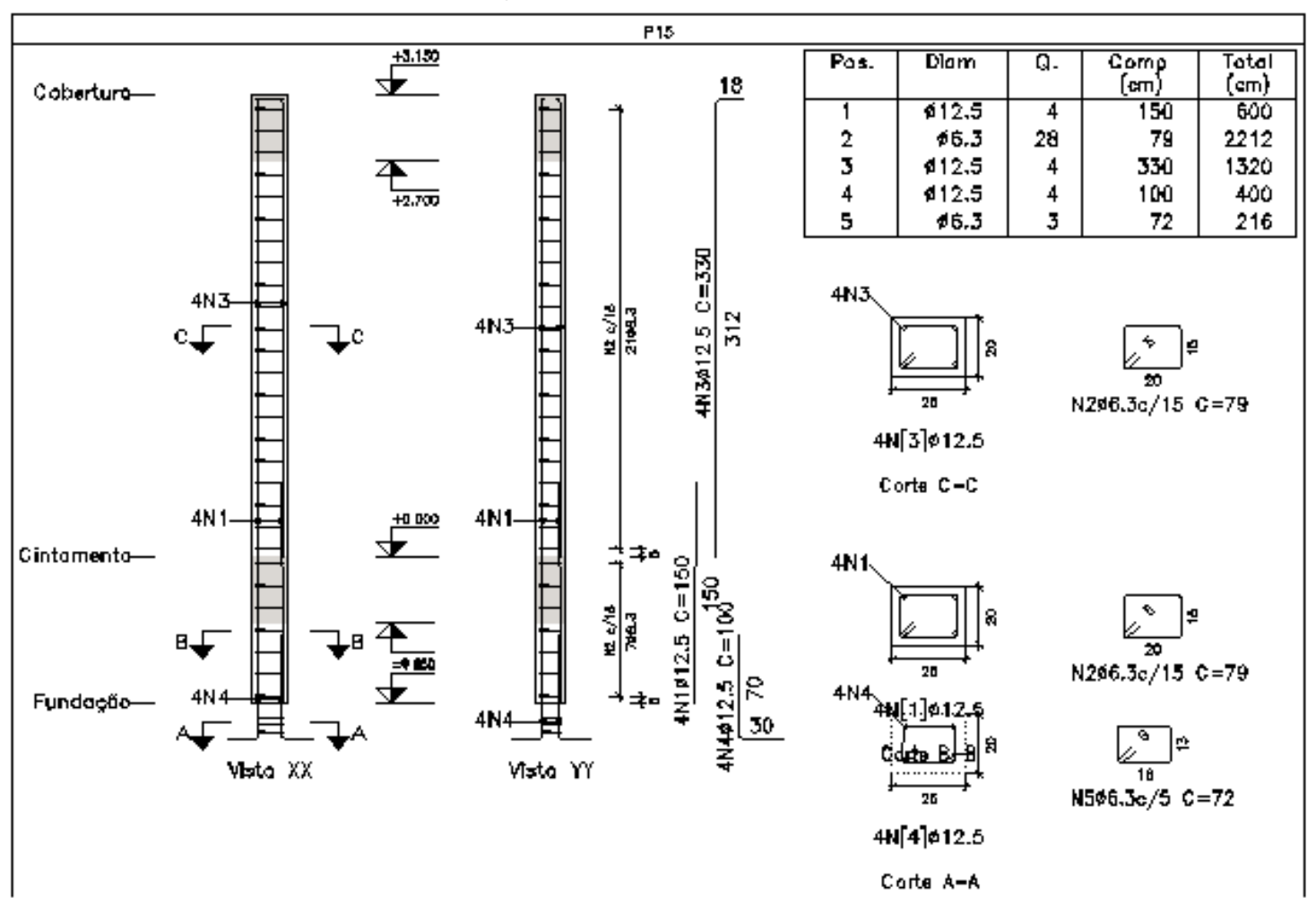

Fonte: Software AutoCAD (2019), 2021 


\section{Pontifícia Universidade Católlca

Figura 68 - Armadura Pilar 16

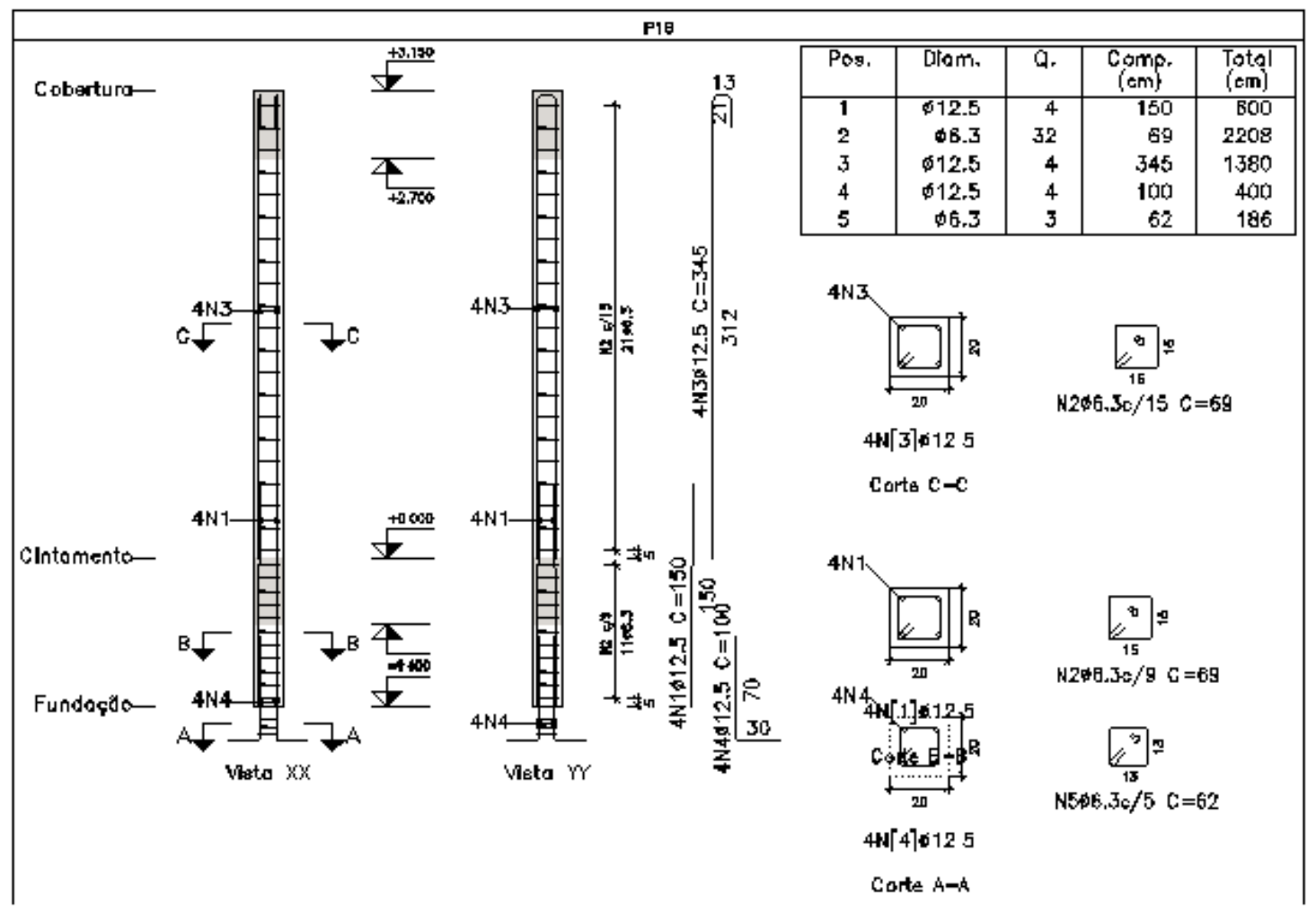

Fonte: Software AutoCAD (2019), 2021

Figura 69 - armadura vigas V1 e V2 - cintamento

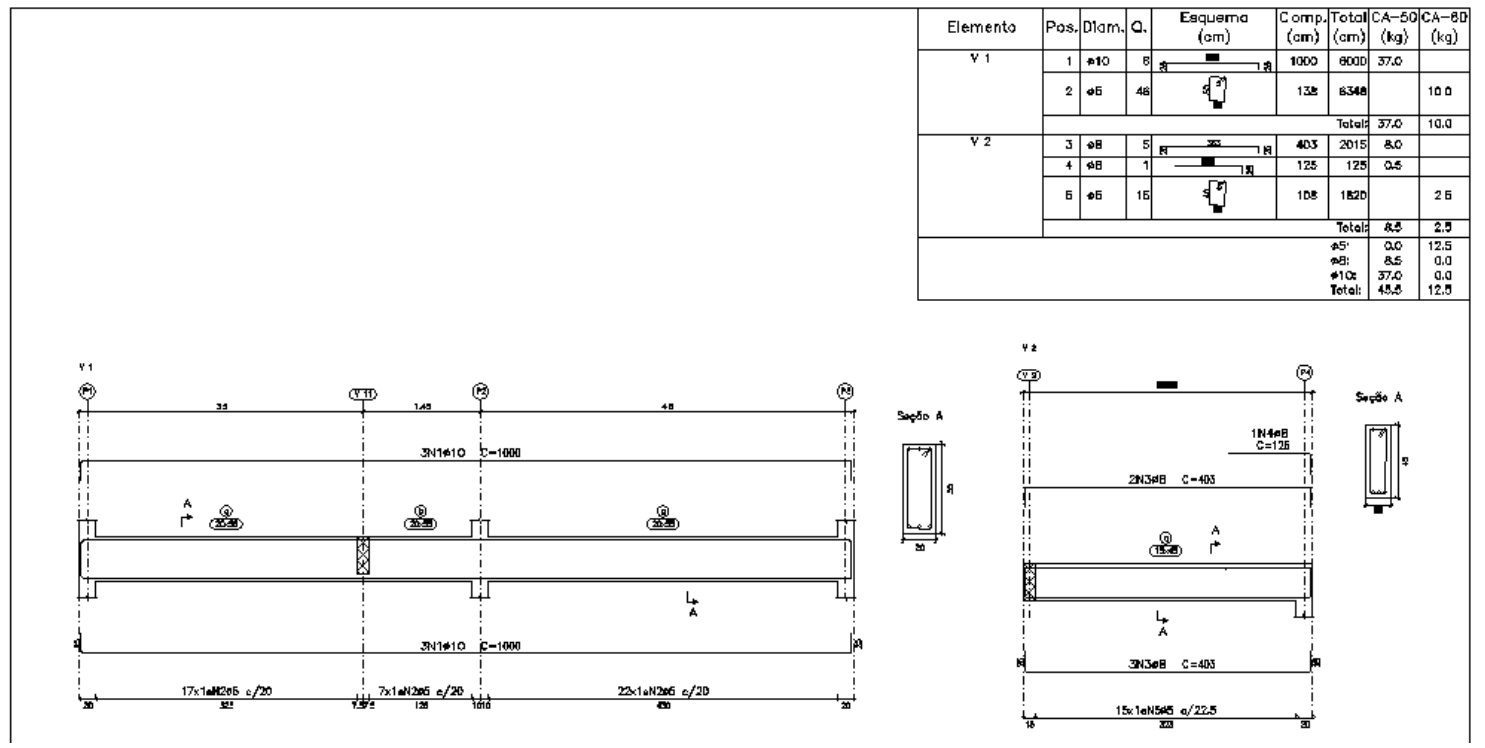

Fonte: Software AutoCAD (2019), 2021 
Figura 70 - Armadura vigas V3, V4 e V5 - cintamento

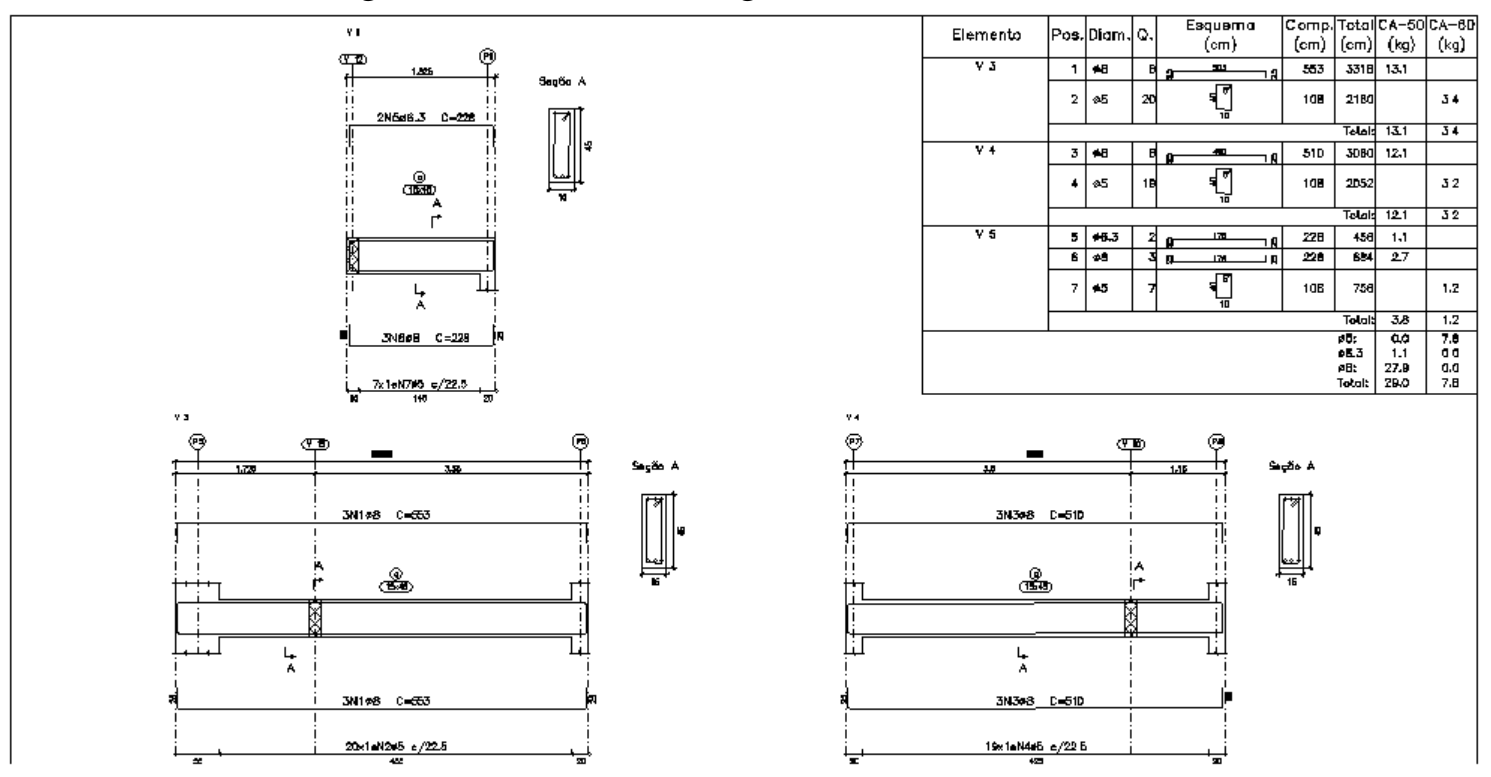

Fonte: Software AutoCAD (2019), 2021

Figura 71 - Armadura vigas V6 e V7 - cintamento

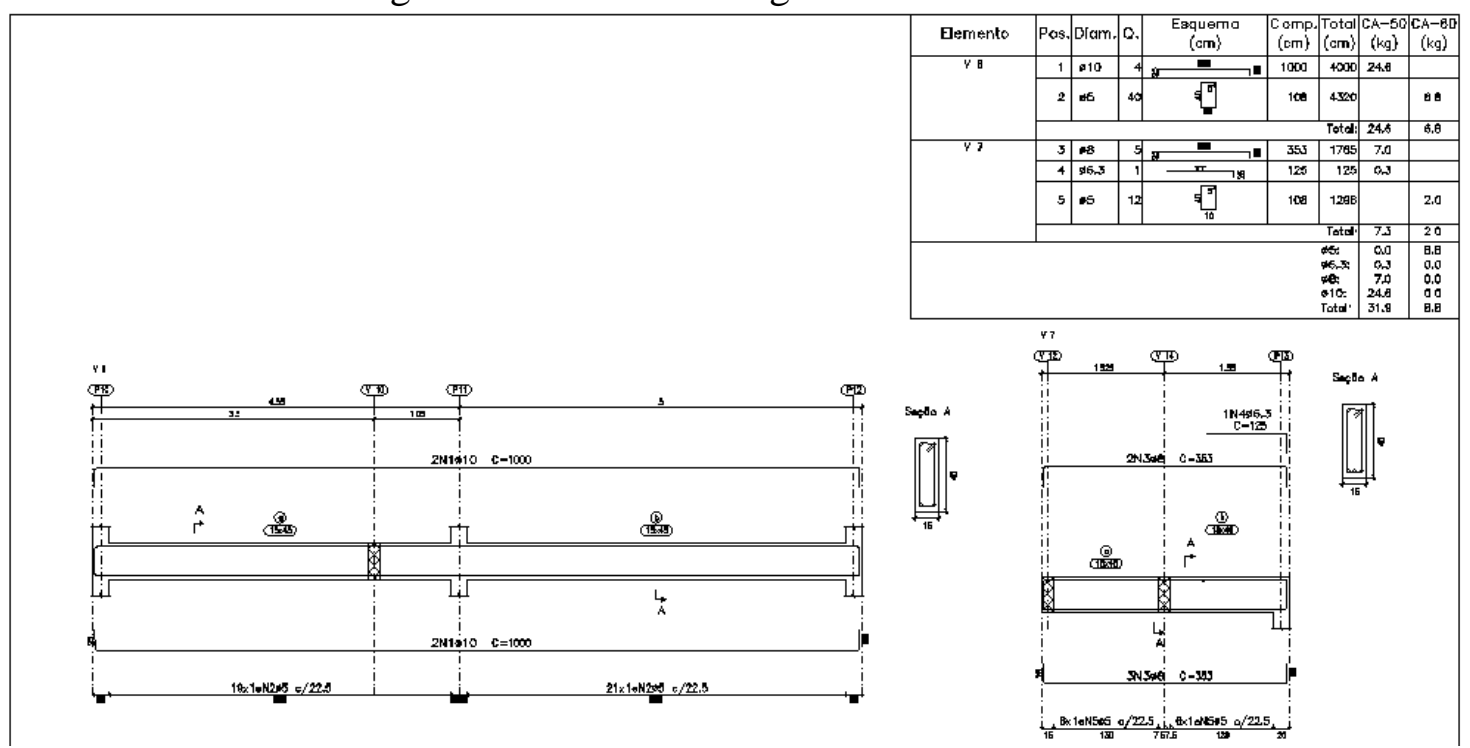

Fonte: Software AutoCAD (2019), 2021 


\section{Pontifícia Universidade Católlica $_{\text {a }}$

Figura 72 - Armadura vigas V8 e V9 - cintamento

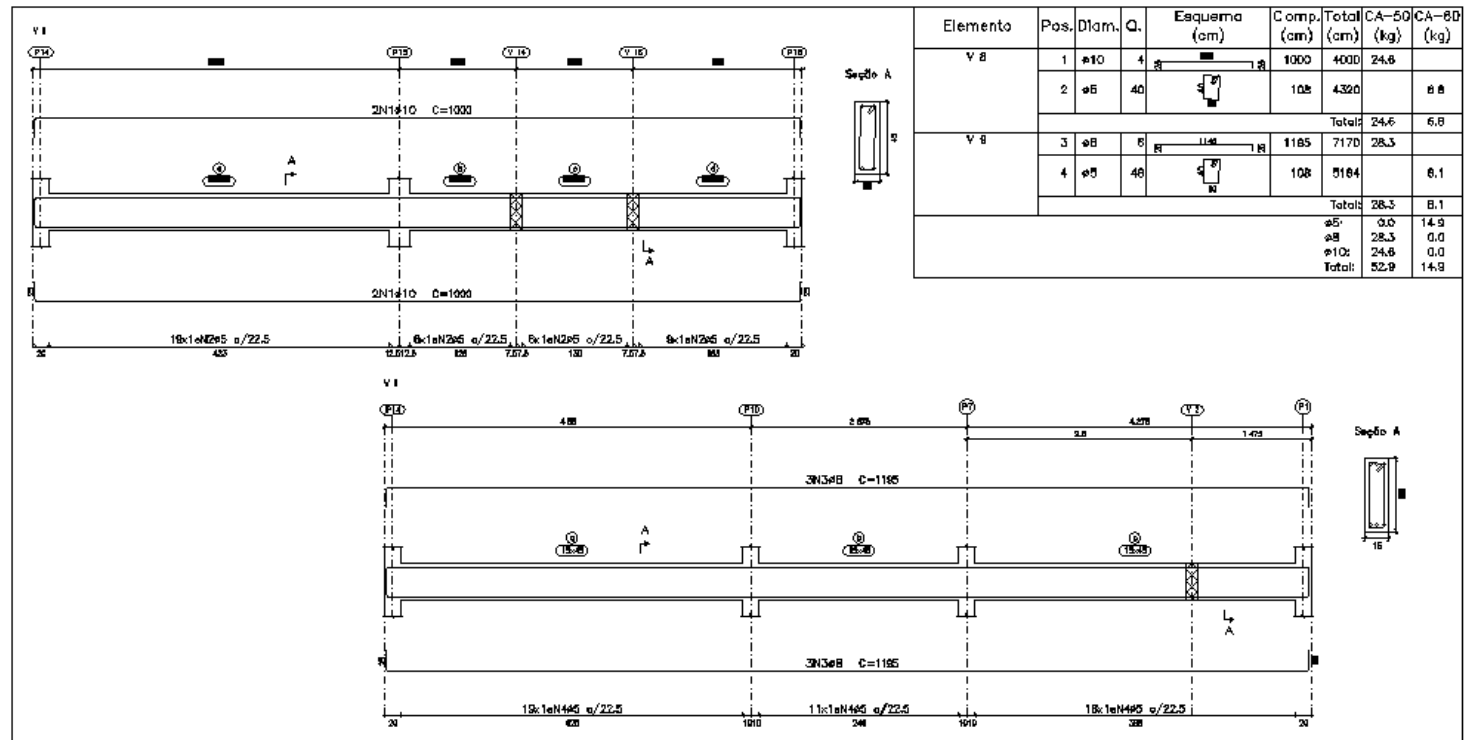

Fonte: Software AutoCAD (2019), 2021

Figura 73 - Armadura vigas V10, V11 e V12 - cintamento

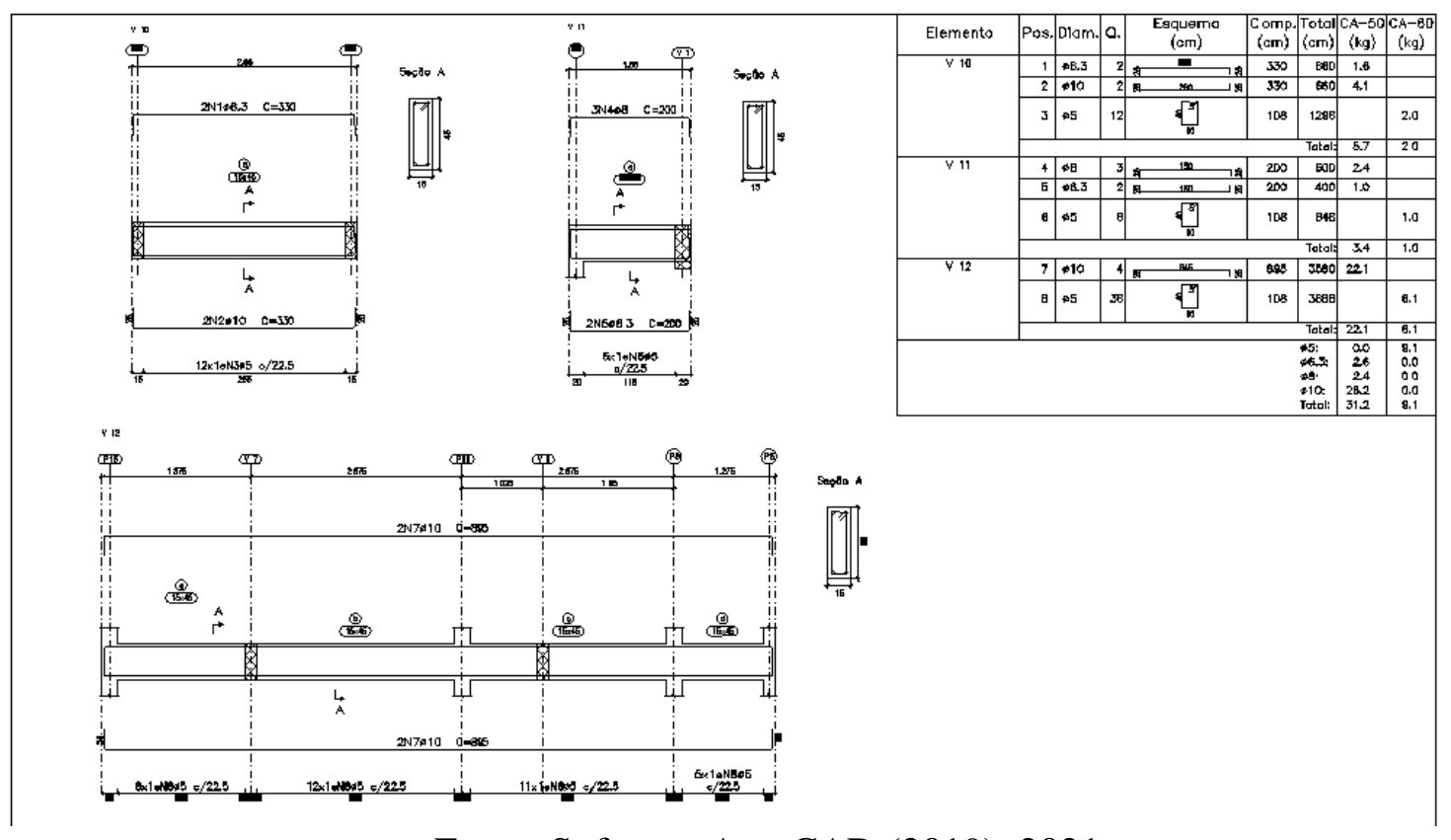

Fonte: Software AutoCAD (2019), 2021 


\section{Pontifícia Universidade Catálica

Figura 74 - armadura vigas V13, V14, V15 e V16 - cintamento

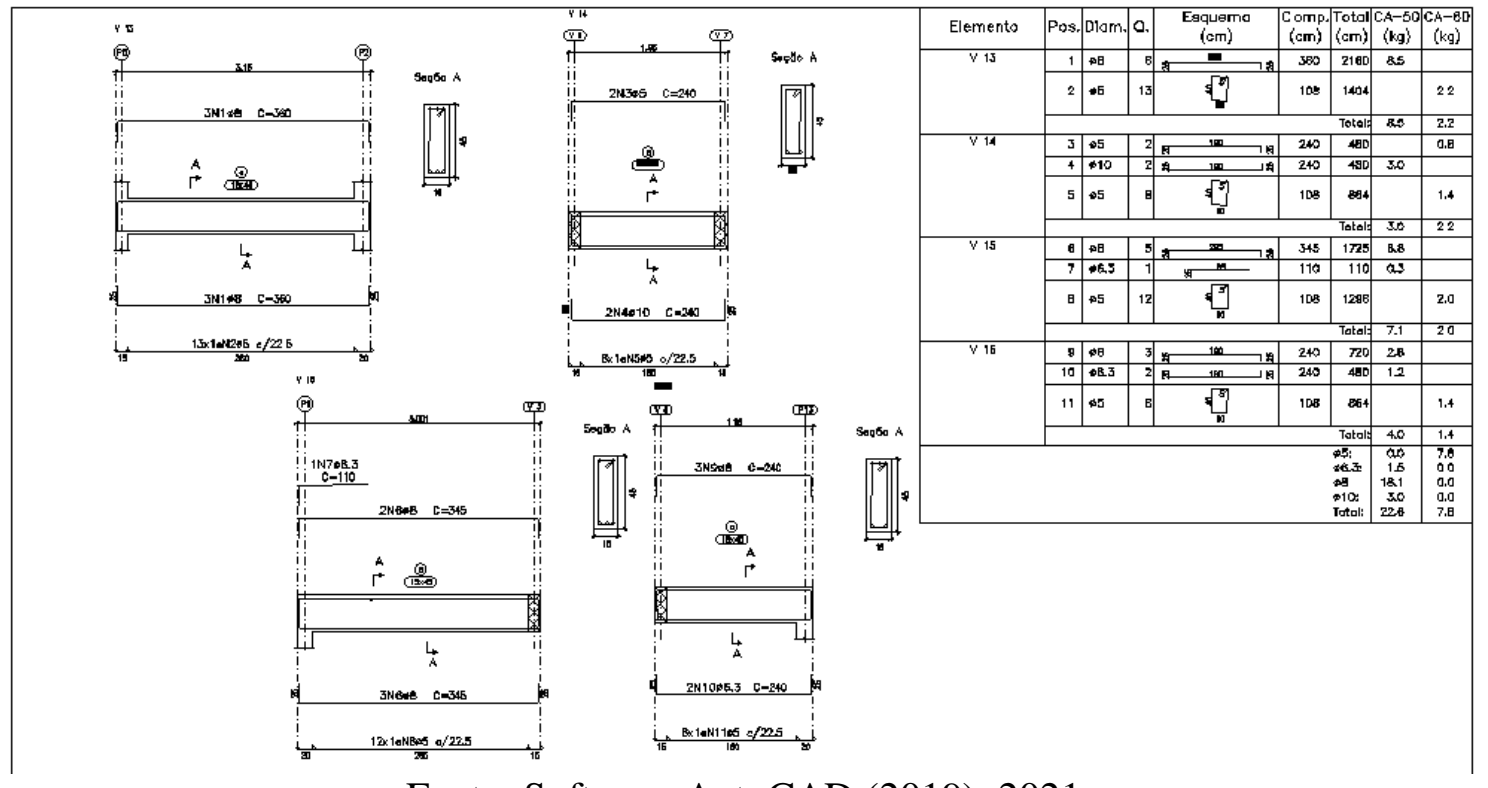

Fonte: Software AutoCAD (2019), 2021

Figura 75 - armadura viga V17 - cintamento

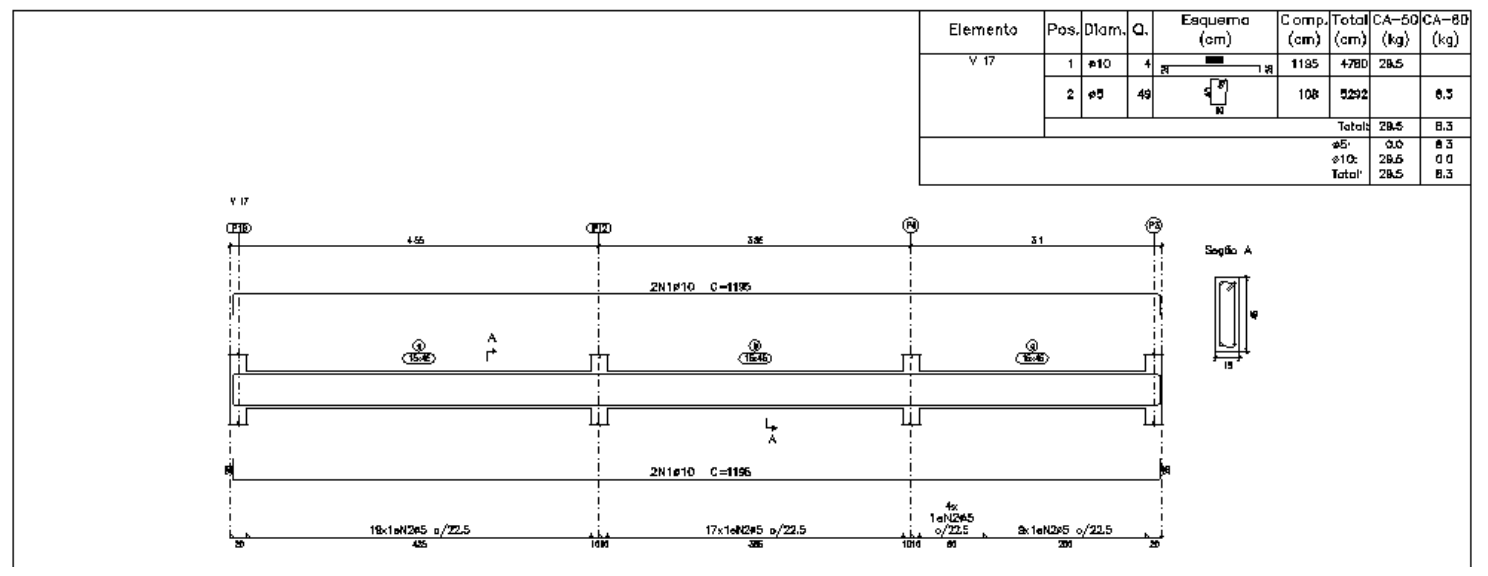

Fonte: Software AutoCAD (2019), 2021 


\section{Pontifícia Universidade Católlica $_{\text {a }}$

Figura 76 - Armadura vigas V1 e V2 - cobertura

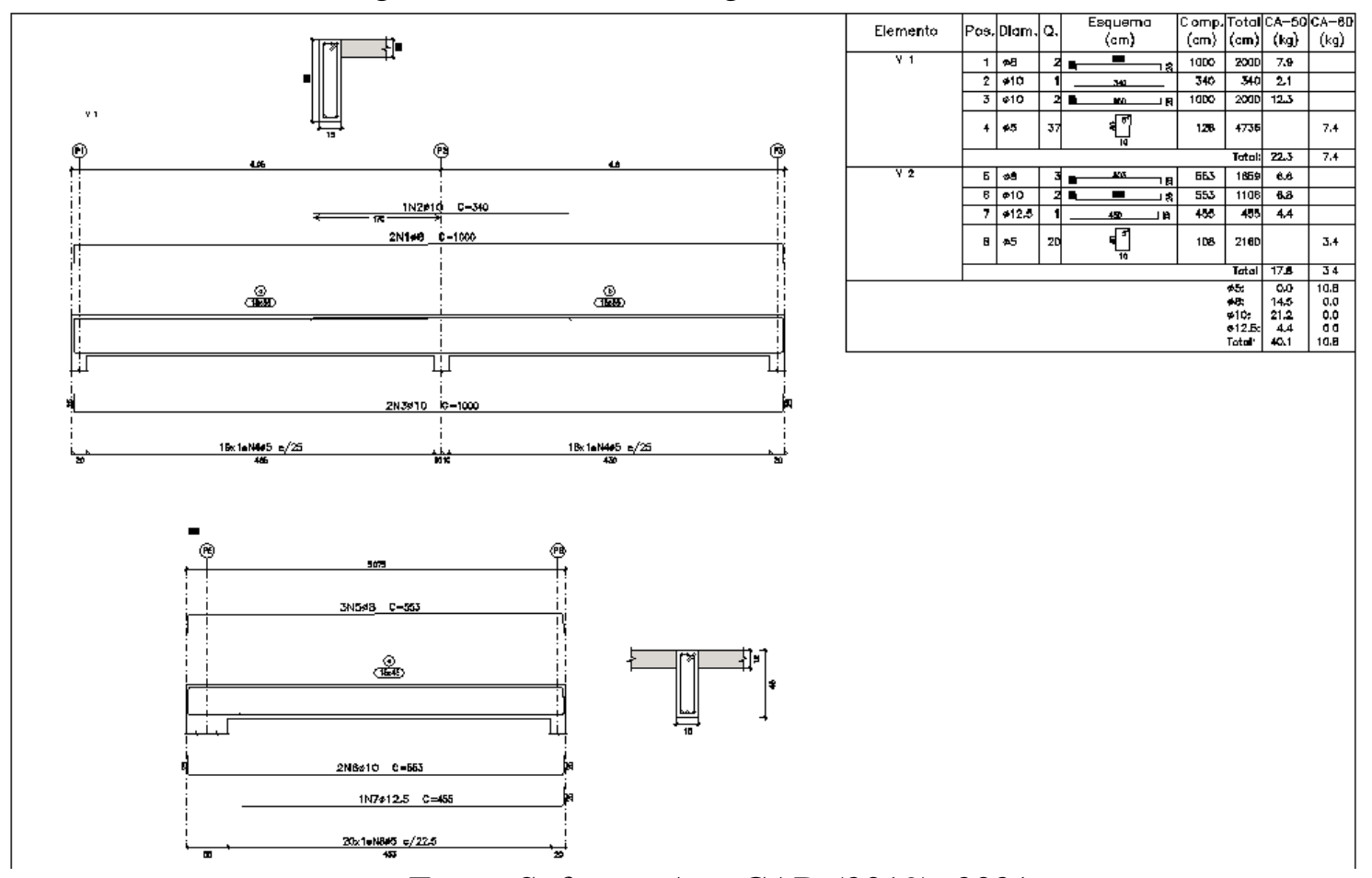

Fonte: Software AutoCAD (2019), 2021

Figura 77 - Armadura vigas V3 e V8 - cobertura

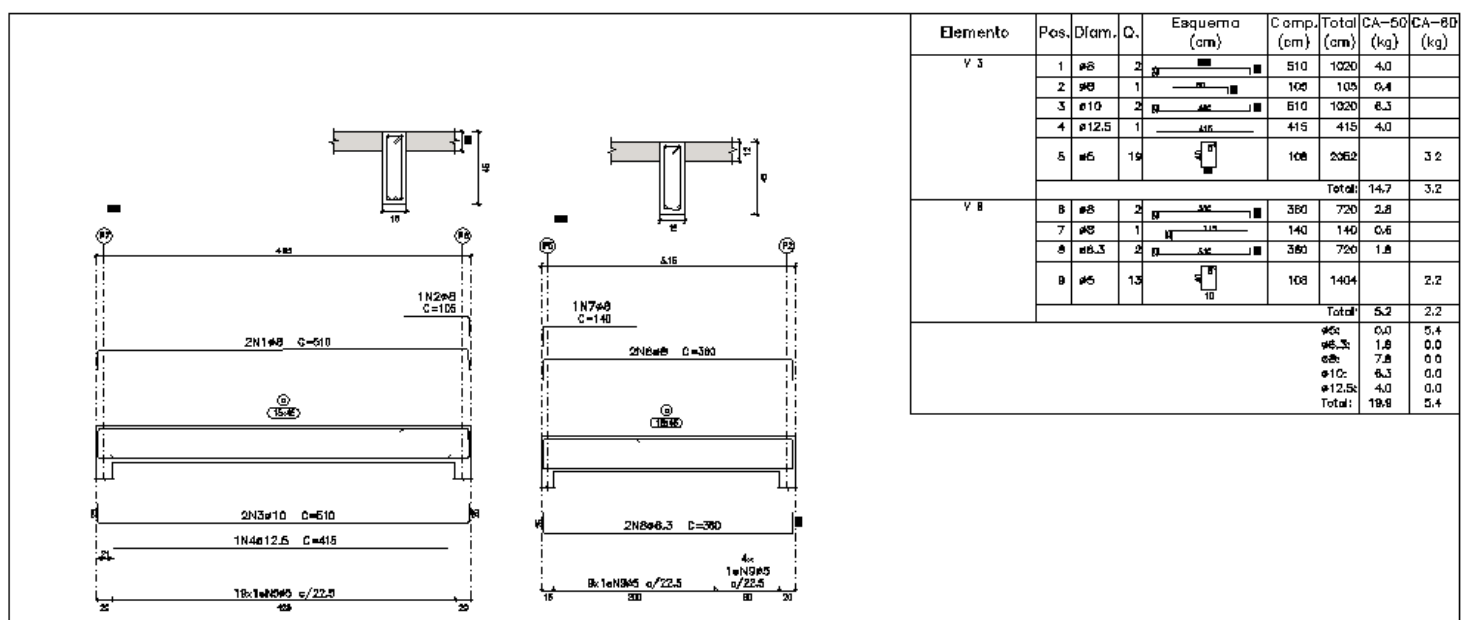

Fonte: Software AutoCAD (2019), 2021 


\section{Pontifícia Universidade Catálica $_{\text {a }}$

Figura 78 - Armadura viga V4 - cobertura

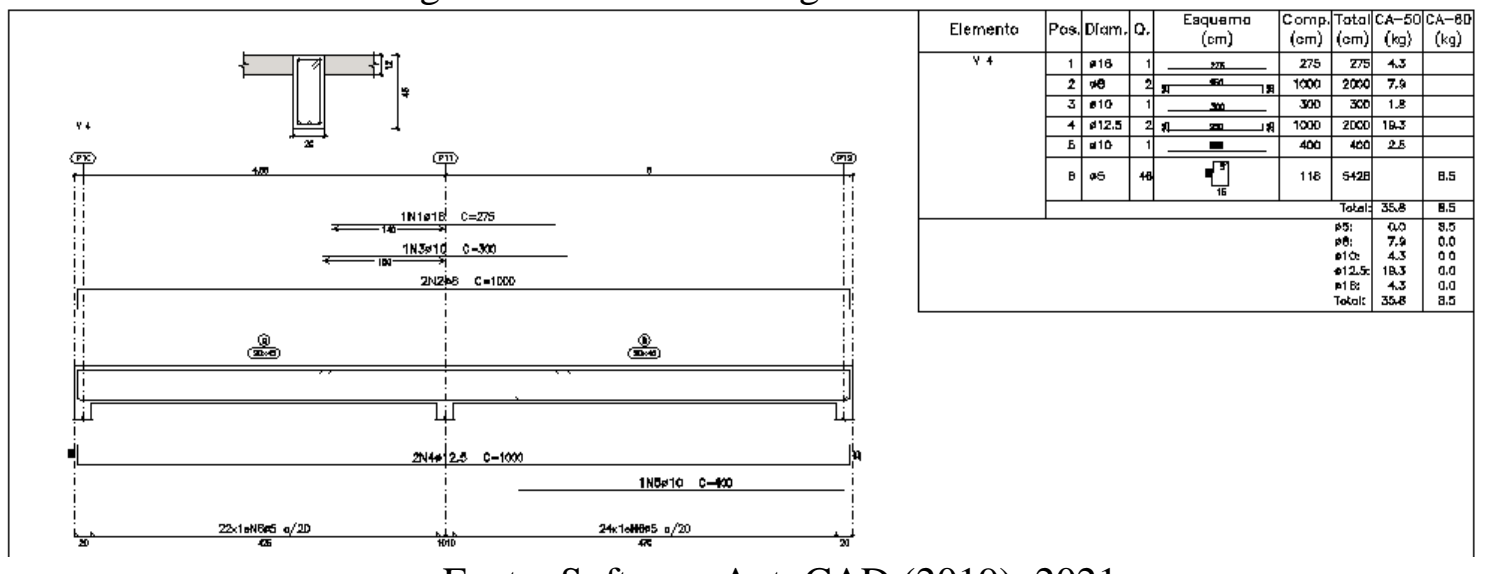

Fonte: Software AutoCAD (2019), 2021

Figura 79 - Armadura viga V5 - cobertura

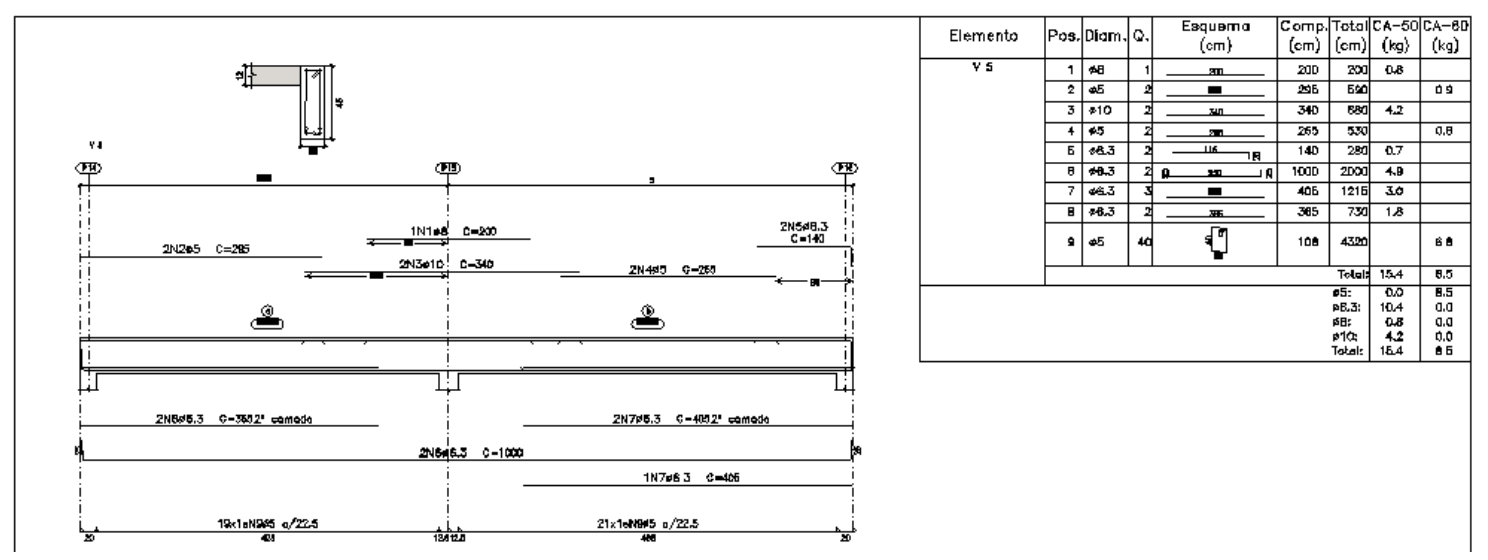

Fonte: Software AutoCAD (2019), 2021 


\section{Pontifícia Universidade Catálica $_{\text {a }}$

Figura 80 - Armadura viga V6 - cobertura

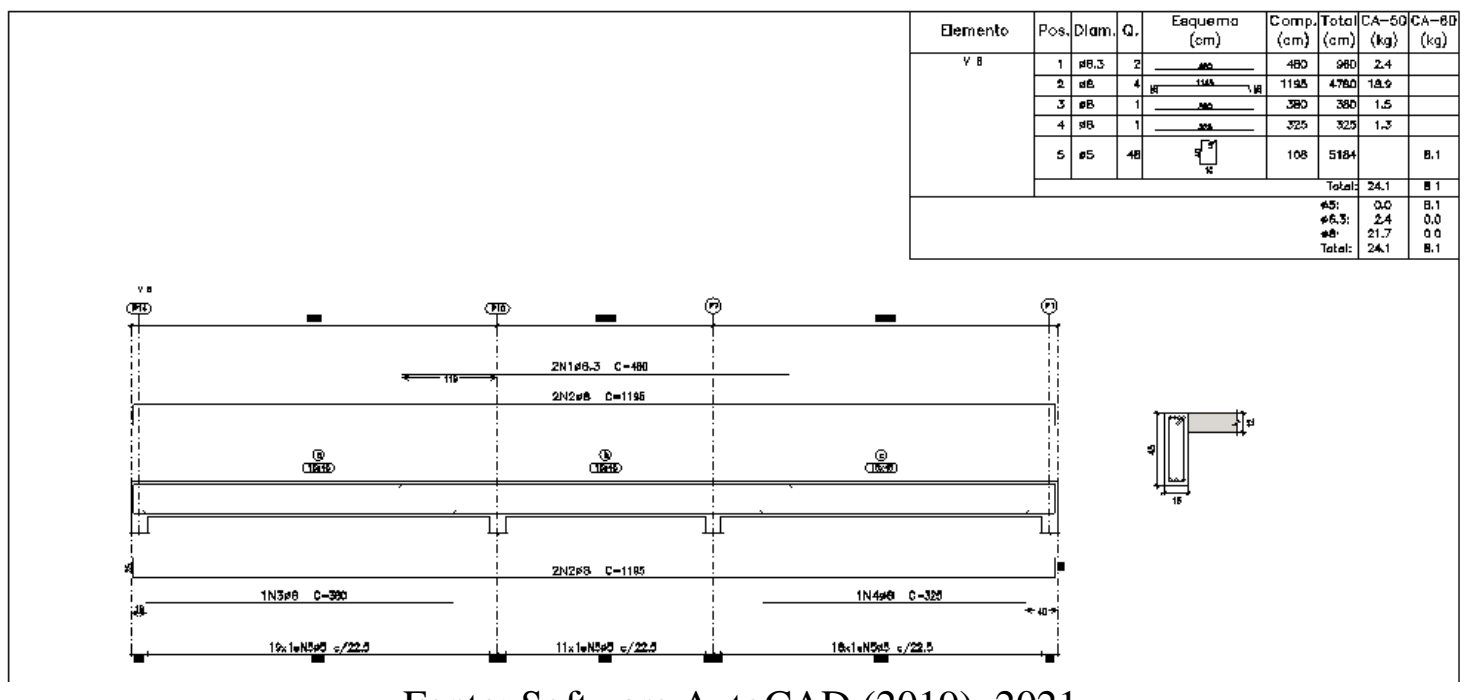

Fonte: Software AutoCAD (2019), 2021

Figura 81 - Armadura viga V7 - cobertura

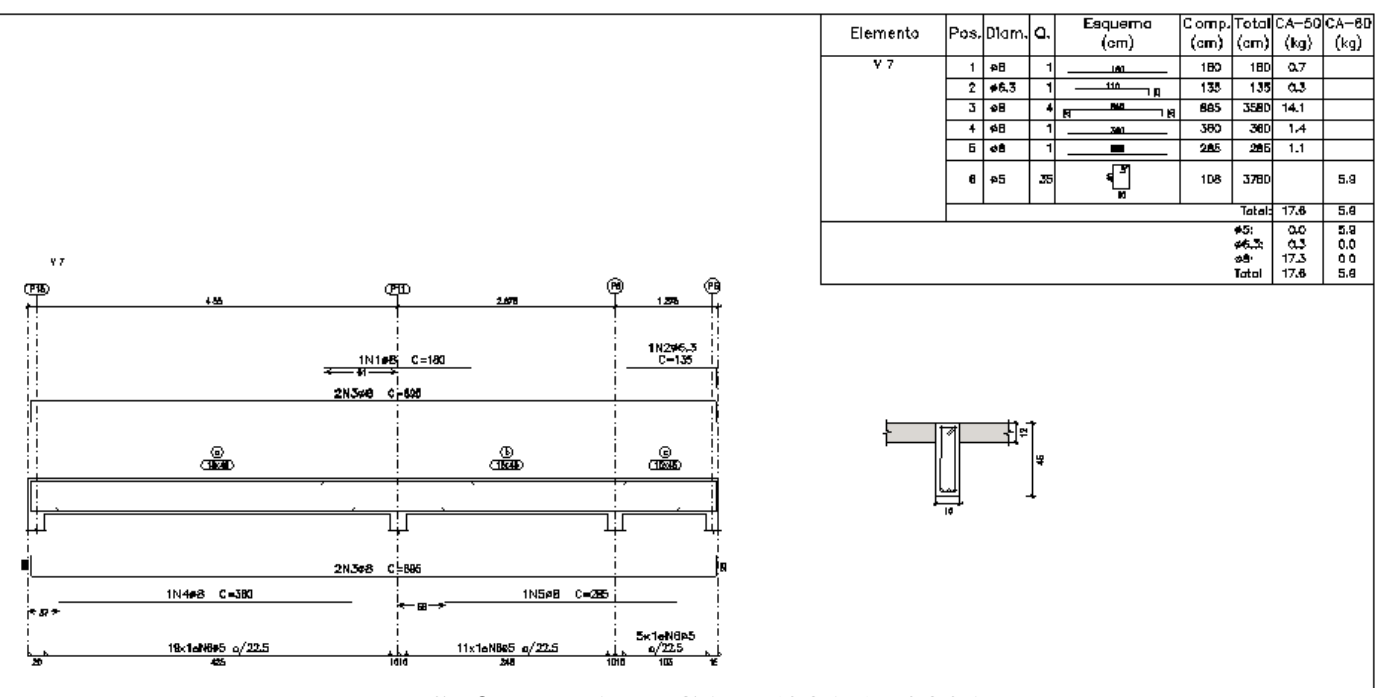

Fonte: Software AutoCAD (2019), 2021 


\section{Pontifícia Universidade Católlica $_{\text {a }}$

Figura 82 - Armadura viga V9 - cobertura

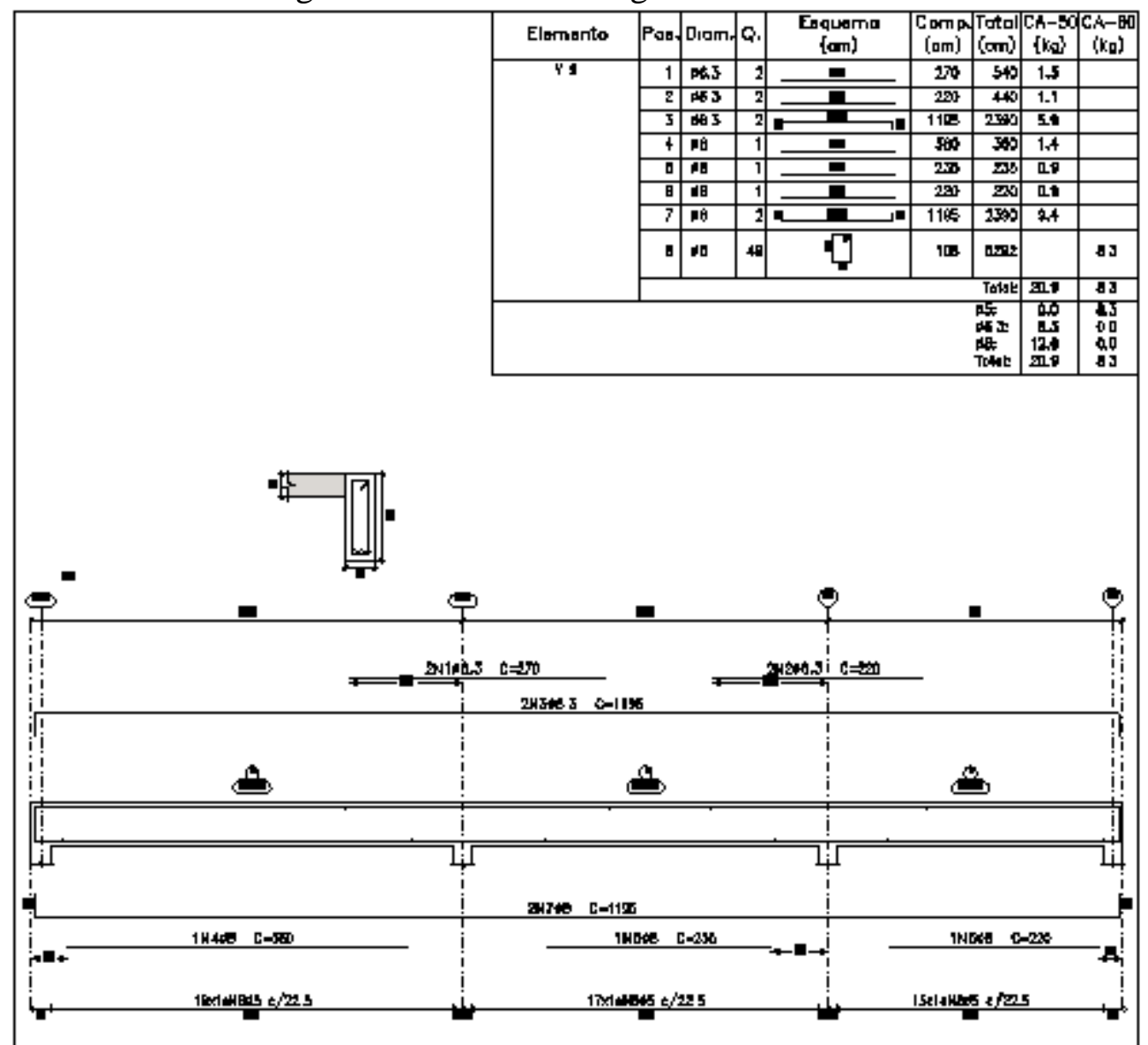

Fonte: Software AutoCAD (2019), 2021 
Figura 83 - Armadura das sapatas referentes aos pilares P1, P2, P6, P7, P8, P10, P12, P13, P14 e P16

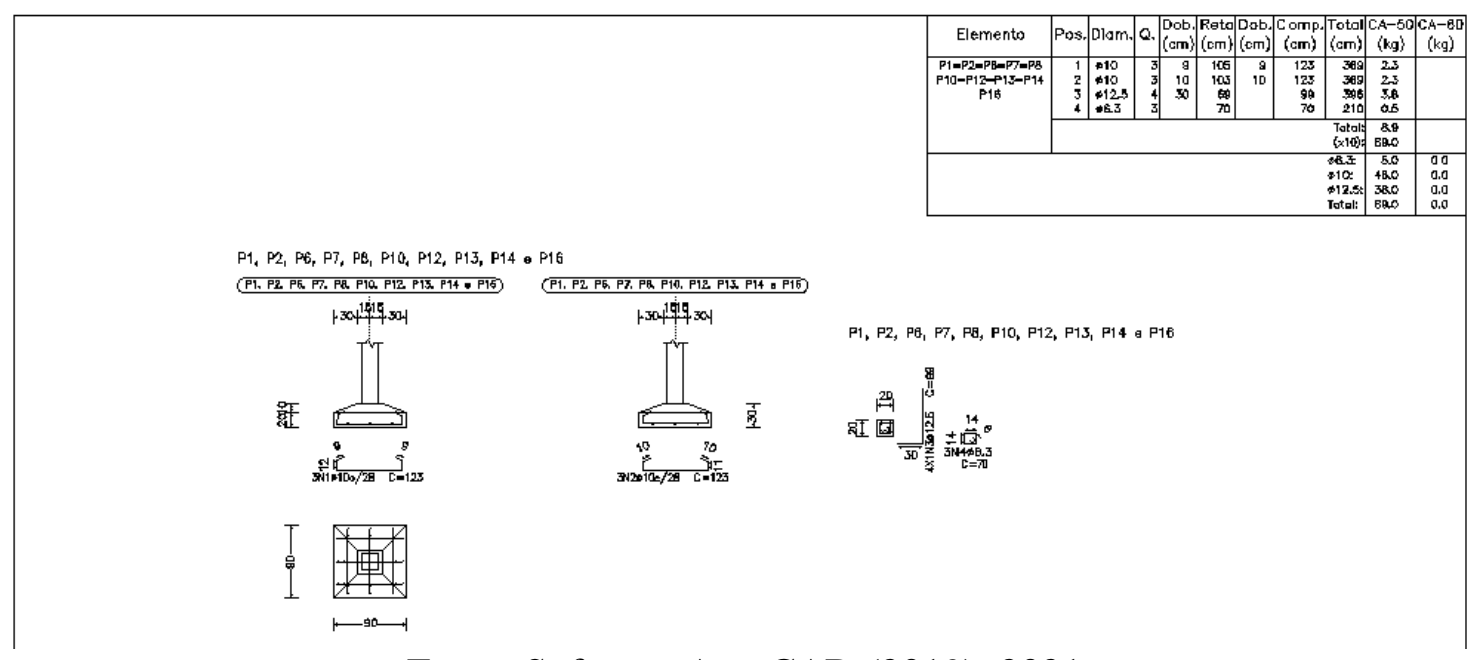

Fonte: Software AutoCAD (2019), 2021

Figura 84 - Armadura das sapatas referentes aos pilares P3, P4 e P9

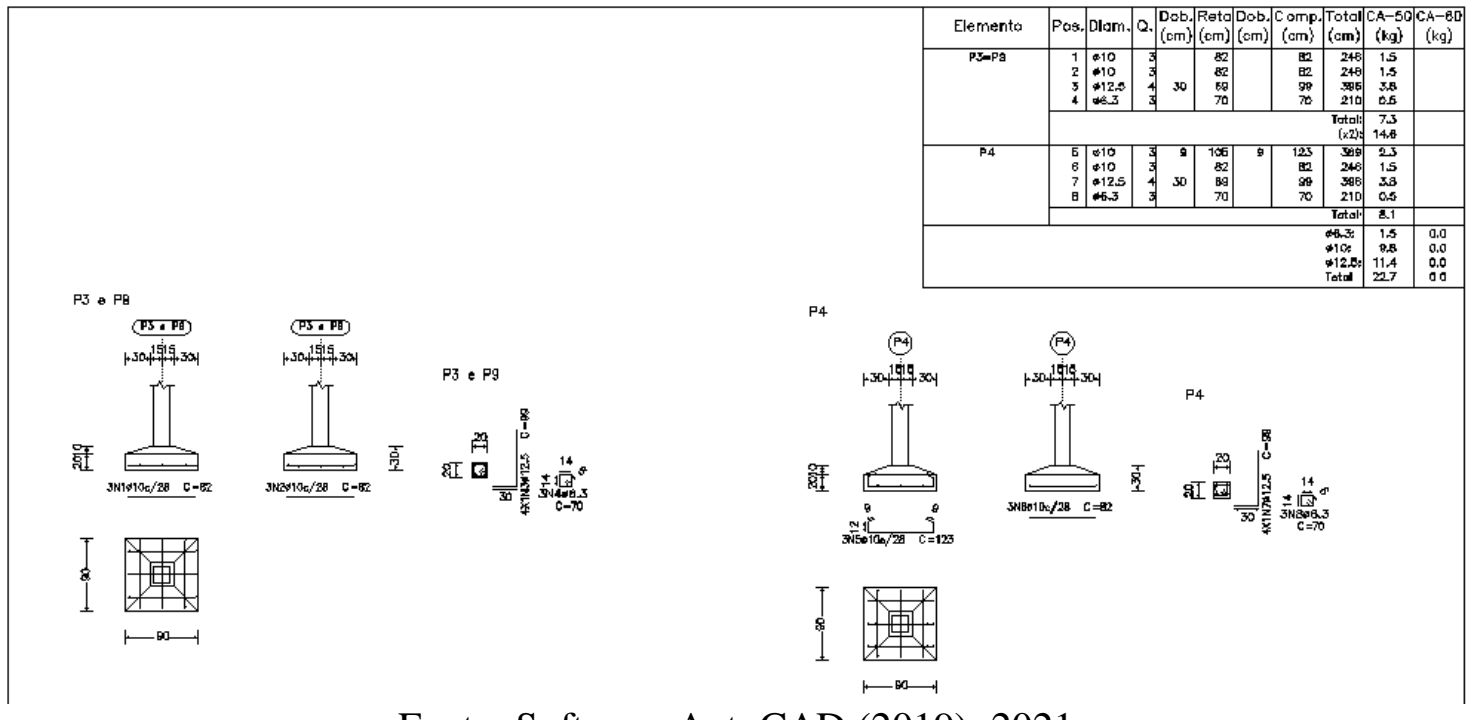

Fonte: Software AutoCAD (2019), 2021 


\section{Pontifícia Universidade Católica \\ DO RIO DE JANEIRO}

Figura 85 - Armadura das sapatas referentes aos pilares P5, P11 e P15

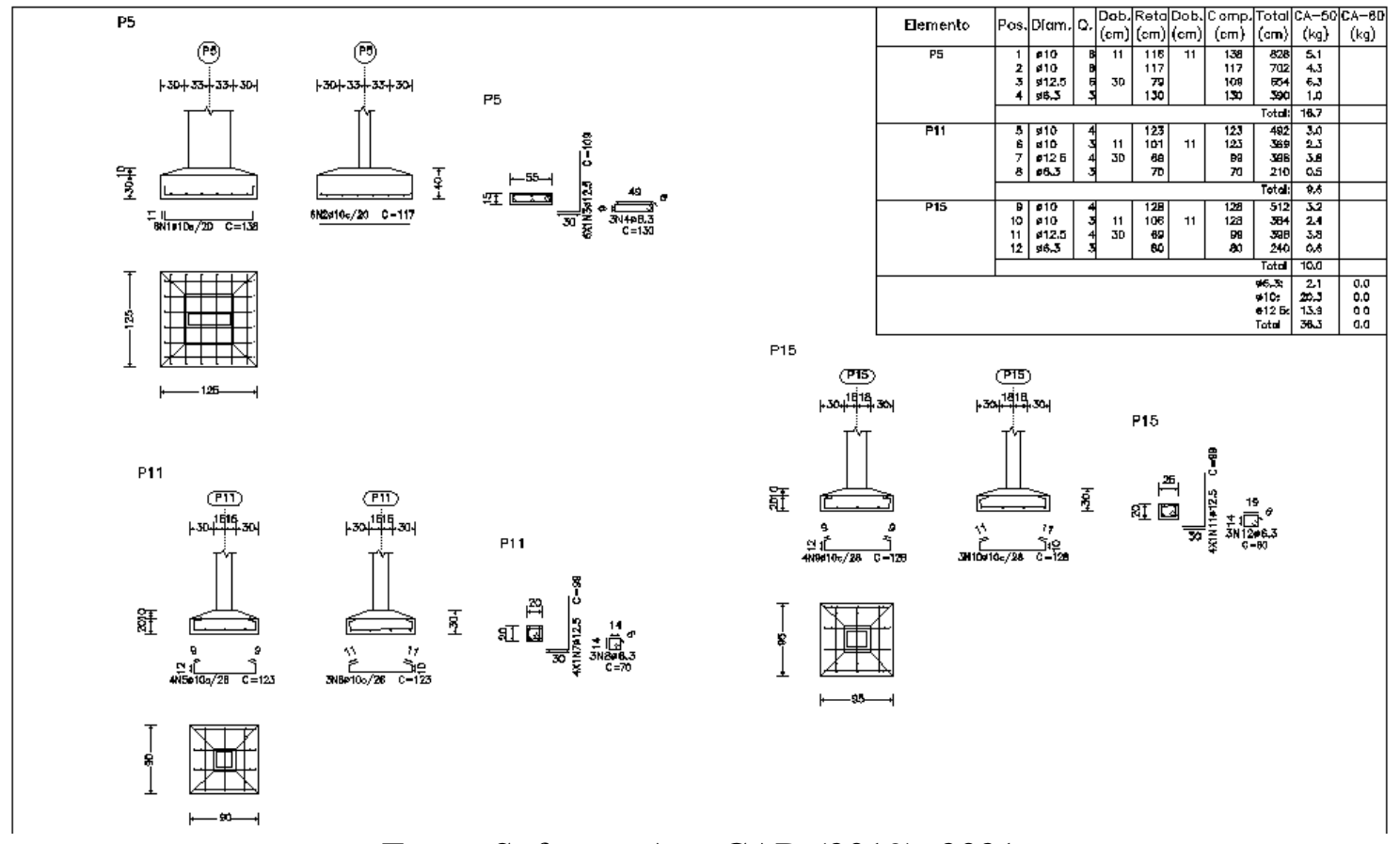

Fonte: Software AutoCAD (2019), 2021

\section{MEMÓRIA DE CÁLCULO RESUMIDA}

Todos os dados deste capítulo foram importados do Cypecad

\section{1.- VERSÃO DO PROGRAMA E NÚMERO DA LICENÇA}

Versão: 2017

Número de licença: 20172

\section{2.- DADOS GERAIS DA ESTRUTURA}

Projeto: Casa em concreto armado de 1 pavimento

Chave: TCC-novo-R1

\section{3.- NORMAS CONSIDERADAS}

Concreto: ABNT NBR 6118:2014

Aços dobrados: ABNT NBR 14762: 2010

Aços laminados e soldados: ABNT NBR 8800:2008

Categoria de uso: Edificações residenciais

\section{4.- AÇÕES CONSIDERADAS}


6.4.1.- Verticais

\begin{tabular}{|l|c|c|}
\hline \multicolumn{1}{|c|}{ Piso } & $\begin{array}{c}\text { S.C.U } \\
\left(\mathrm{kN} / \mathrm{m}^{2}\right)\end{array}$ & $\begin{array}{c}\text { C. permanentes } \\
\left(\mathrm{kN} / \mathrm{m}^{2}\right)\end{array}$ \\
\hline Cobertura & 1.5 & 0.0 \\
\hline Cintamento & 1.5 & 0.5 \\
\hline Fundação & 0.0 & 0.0 \\
\hline
\end{tabular}

6.4.2.- Vento

Sem ação de vento

\subsection{3.- Sismo}

Sem ação de sismo

\subsection{4.- Hipóteses/ações de carga}

\begin{tabular}{|l|l|}
\hline Automáticas & $\begin{array}{l}\text { Peso próprio } \\
\text { Cargas permanentes } \\
\text { Sobrecarga }\end{array}$ \\
\hline
\end{tabular}

\subsection{5.- Relatório de cargas}

Cargas especiais introduzidas (em $\mathrm{kN}, \mathrm{kN} / \mathrm{m}$ e $\mathrm{kN} / \mathrm{m}^{2}$ )

\begin{tabular}{|c|c|c|c|c|}
\hline Grupo & Hipótese & Tipo & Valor & Coordenadas \\
\hline \multirow{21}{*}{ Cintamento } & Cargas permanentes & Linear & 5.30 & $(2.03,12.00)(6.88,12.00)$ \\
\hline & Cargas permanentes & Linear & 5.30 & $(6.88,9.00)(6.88,11.98)$ \\
\hline & Cargas permanentes & Linear & 5.30 & $(11.38,9.00)(6.68,9.01)$ \\
\hline & Cargas permanentes & Linear & 5.30 & $(2.03,7.80)(2.03,11.98)$ \\
\hline & Cargas permanentes & Linear & 5.30 & $(2.03,5.13)(2.03,7.80)$ \\
\hline & Cargas permanentes & Linear & 5.30 & $(2.03,0.68)(2.03,5.13)$ \\
\hline & Cargas permanentes & Linear & 5.30 & $(2.03,0.65)(6.48,0.65)$ \\
\hline & Cargas permanentes & Linear & 5.30 & $(6.48,0.65)(7.93,0.65)$ \\
\hline & Cargas permanentes & Linear & 5.30 & $(7.93,0.65)(9.38,0.65)$ \\
\hline & Cargas permanentes & Linear & 5.30 & $(9.38,0.65)(11.38,0.65)$ \\
\hline & Cargas permanentes & Linear & 5.30 & $(6.48,0.68)(6.48,5.13)$ \\
\hline & Cargas permanentes & Linear & 5.30 & $(2.03,5.10)(6.48,5.10)$ \\
\hline & Cargas permanentes & Linear & 5.30 & $(2.03,7.80)(6.48,7.80)$ \\
\hline & Cargas permanentes & Linear & 5.30 & $(6.48,5.13)(6.48,7.80)$ \\
\hline & Cargas permanentes & Linear & 5.30 & $(6.48,5.10)(11.38,5.10)$ \\
\hline & Cargas permanentes & Linear & 5.30 & $(11.38,0.68)(11.38,5.13)$ \\
\hline & Cargas permanentes & Linear & 5.30 & $(11.38,5.13)(11.38,8.98)$ \\
\hline & Cargas permanentes & Linear & 5.30 & $(6.48,7.80)(6.48,9.00)$ \\
\hline & Cargas permanentes & Linear & 5.90 & $(2.03,10.60)(5.43,10.60)$ \\
\hline & Cargas permanentes & Linear & 5.90 & $(5.43,10.63)(5.43,11.98)$ \\
\hline & Cargas permanentes & Linear & 5.90 & $(5.43,5.10)(5.43,7.80)$ \\
\hline
\end{tabular}




\begin{tabular}{|c|c|c|c|c|}
\hline Grupo & Hipótese & Tipo & Valor & Coordenadas \\
\hline & Cargas permanentes & Linear & 5.90 & $(6.48,6.15)(8.13,6.15)$ \\
\hline & Cargas permanentes & Linear & 5.90 & $(8.13,6.18)(8.13,9.01)$ \\
\hline & Cargas permanentes & Linear & 5.90 & $(6.48,2.45)(7.93,2.45)$ \\
\hline & Cargas permanentes & Linear & 5.90 & $(7.93,2.45)(9.38,2.45)$ \\
\hline & Cargas permanentes & Linear & 5.90 & $(7.93,0.65)(7.93,2.45)$ \\
\hline & Cargas permanentes & Linear & 5.90 & $(9.38,0.65)(9.38,2.43)$ \\
\hline \multirow[b]{5}{*}{ Cobertura } & Cargas permanentes & Linear & 1.95 & $(2.03,11.98)(6.88,11.98)$ \\
\hline & Cargas permanentes & Linear & 1.95 & $(6.88,11.98)(11.38,11.98)$ \\
\hline & Cargas permanentes & Linear & 1.95 & $(2.03,0.65)(6.48,0.65)$ \\
\hline & Cargas permanentes & Linear & 1.95 & $(6.48,0.65)(11.38,0.65)$ \\
\hline & Cargas permanentes & Superficial & 11.40 & $\begin{array}{l}(7.09,10.22)(7.01,9.86)(7.07,9.58) \\
(7.18,9.39)(7.37,9.23)(7.56,9.15) \\
(7.84,9.13)(8.15,9.23)(8.44,9.57) \\
(8.50,9.83)(8.45,10.17)(8.27,10.42) \\
(8.06,10.56)(7.81,10.62)(7.56,10.60) \\
(7.34,10.50)(7.19,10.37)\end{array}$ \\
\hline
\end{tabular}

\section{5.- ESTADOS LIMITES}

\begin{tabular}{|l|l|}
\hline $\begin{array}{l}\text { E.L.U. Concreto } \\
\text { E.L.Util Fissuração. Concreto } \\
\text { E.L.U. Concreto em fundações }\end{array}$ & ABNT NBR 6118:2014(ELU) \\
\hline $\begin{array}{l}\text { Tensões sobre o terreno } \\
\text { Deslocamentos }\end{array}$ & Ações características \\
\hline
\end{tabular}

\section{6.- SITUAÇÕES DE PROJETO}

Para as distintas situações de projeto, as combinações de ações serão definidas de acordo com os seguintes critérios:

\section{- Com coeficientes de combinação}

\section{- Sem coeficientes de combinação}

- Onde:

$\mathrm{G}_{\mathrm{k}}$ Ação permanente 
$P_{k} \quad$ Acção de pré-esforço

$Q_{k}$ Ação variável

$\gamma_{G}$ Coeficiente parcial de segurança das ações permanentes

$\gamma_{P}$ Coeficiente parcial de segurança da acção de pré-esforço

$\gamma_{Q, 1}$ Coeficiente parcial de segurança da ação variável principal

$\gamma_{Q, i}$ Coeficiente parcial de segurança das ações variáveis de acompanhamento

$\psi_{p, 1}$ Coeficiente de combinação da ação variável principal

$\psi_{a, i}$ Coeficiente de combinação das ações variáveis de acompanhamento

6.6.1.- Coeficientes parciais de segurança $(\gamma)$ e coeficientes de combinação $(\psi)$ Para cada situação de projeto e estado limite, os coeficientes a utilizar serão:

E.L.U. Concreto: ABNT NBR 6118:2014

E.L.U. Concreto em fundações: ABNT NBR 6118:2014

\begin{tabular}{|l|c|c|c|c|}
\hline \multicolumn{5}{|c|}{ Situação 1 } \\
\hline & \multicolumn{2}{|c|}{$\begin{array}{c}\text { Coeficientes parciais de segurança }(\gamma) \\
\text { Favorável }\end{array}$} & $\begin{array}{c}\text { Coeficientes de combinação }(\psi) \\
\text { Desfavorável }\end{array}$ & $\begin{array}{c}\text { Principal }\left(\psi_{\mathrm{p}}\right) \\
\text { Acompanhamento }\left(\psi_{\mathrm{a}}\right)\end{array}$ \\
\hline Permanente (G) & 1.000 & 1.400 & - & - \\
Sobrecarga (Q) & 0.000 & 1.400 & 1.000 & 0.500 \\
\hline
\end{tabular}

\section{E.L.Util Fissuração. Concreto: ABNT NBR 6118:2014}

\begin{tabular}{|l|c|c|c|c|}
\hline \multicolumn{4}{|c|}{ Situação 1 } \\
\hline & \multicolumn{2}{|c|}{$\begin{array}{c}\text { Coeficientes parciais de segurança }(\gamma) \\
\text { Favorável }\end{array}$} & $\begin{array}{c}\text { Coeficientes de combinação }(\psi) \\
\text { Desfavorável }\end{array}$ & $\begin{array}{l}\text { Principal }\left(\psi_{\mathrm{p}}\right) \\
\text { Acompanhamento }\left(\psi_{\mathrm{a}}\right)\end{array}$ \\
\hline Permanente (G) & 1.000 & 1.000 & - & - \\
Sobrecarga (Q) & 0.000 & 1.000 & 0.400 & 0.300 \\
\hline
\end{tabular}

Tensões sobre o terreno

\begin{tabular}{|l|c|c|}
\hline \multicolumn{3}{|c|}{ Ações variáveis sem sismo } \\
\hline \multirow{2}{*}{} & \multicolumn{2}{|c|}{ Coeficientes parciais de segurança $(\gamma)$} \\
\cline { 2 - 3 } & Favorável & Desfavorável \\
\hline Permanente $(G)$ & 1.000 & 1.000 \\
Sobrecarga $(Q)$ & 0.000 & 1.000 \\
\hline
\end{tabular}

\section{Deslocamentos}

\begin{tabular}{|l|c|c|}
\hline \multicolumn{3}{|c|}{ Ações variáveis sem sismo } \\
\hline \multirow{2}{*}{} & \multicolumn{2}{|c|}{ Coeficientes parciais de segurança $(\gamma)$} \\
\cline { 2 - 3 } & Favorável & Desfavorável \\
\hline Permanente (G) & 1.000 & 1.000 \\
Sobrecarga (Q) & 0.000 & 1.000 \\
\hline
\end{tabular}


6.6.2.- Combinações

- Nomes das ações

PP Peso próprio

CP Cargas permanentes

Qa Sobrecarga

- E.L.U. Concreto

- E.L.U. Concreto em fundações

\begin{tabular}{|c|c|c|c|}
\hline Comb. & PP & CP & Qa \\
\hline 1 & 1.000 & 1.000 & \\
\hline 2 & 1.400 & 1.400 & \\
\hline 3 & 1.000 & 1.000 & 1.400 \\
\hline 4 & 1.400 & 1.400 & 1.400 \\
\hline
\end{tabular}

- E.L.Util Fissuração. Concreto

\begin{tabular}{|c|c|c|c|}
\hline Comb. & PP & CP & Qa \\
\hline 1 & 1.000 & 1.000 & \\
\hline 2 & 1.000 & 1.000 & 0.400 \\
\hline
\end{tabular}

- Tensões sobre o terreno

- Deslocamentos

\begin{tabular}{|c|c|c|c|}
\hline Comb. & PP & CP & Qa \\
\hline 1 & 1.000 & 1.000 & \\
\hline 2 & 1.000 & 1.000 & 1.000 \\
\hline
\end{tabular}

\section{7.- DADOS GEOMÉTRICOS DE GRUPOS E PISOS}

\begin{tabular}{|r|l|r|r|r|r|}
\hline Grupo & Nome do grupo & Piso & Nome piso & Altura & Cota \\
\hline 2 & Cobertura & 2 & Cobertura & 3.15 & 3.15 \\
\hline 1 & Cintamento & 1 & Cintamento & 1.00 & 0.00 \\
\hline 0 & Fundação & & & & -1.00 \\
\hline
\end{tabular}

\section{8.- DADOS GEOMÉTRICOS DE PILARES, PILARES-PAREDES E CORTINAS}

\section{1.- Pilares}

GI: grupo inicial

GF: grupo final

Ang: ângulo do pilar em graus sexagesimais 


\begin{tabular}{|l|c|c|c|c|c|c|}
\hline Referência & Coord(P.Fixo) & GI- GF & Vinculação exterior & Ang. & Ponto fixo & Altura de apoio \\
\hline P1 & $(2.03,11.98)$ & $0-2$ & Com vinculação exterior & 0.0 & Centro & 0.30 \\
\hline P2 & $(6.88,11.98)$ & $0-2$ & Com vinculação exterior & 0.0 & Centro & 0.30 \\
\hline P3 & $(11.38,11.98)$ & $0-2$ & Com vinculação exterior & 0.0 & Centro & 0.30 \\
\hline P4 & $(5.43,10.53)$ & $0-1$ & Com vinculação exterior & 0.0 & Centro & 0.30 \\
\hline P5 & $(6.68,9.00)$ & $0-2$ & Com vinculação exterior & 0.0 & Centro & 0.40 \\
\hline P6 & $(11.38,8.98)$ & $0-2$ & Com vinculação exterior & 0.0 & Centro & 0.30 \\
\hline P7 & $(2.03,7.80)$ & $0-2$ & Com vinculação exterior & 0.0 & Centro & 0.30 \\
\hline P8 & $(6.48,7.80)$ & $0-2$ & Com vinculação exterior & 0.0 & Centro & 0.30 \\
\hline P9 & $(8.13,6.28)$ & $0-1$ & Com vinculação exterior & 0.0 & Centro & 0.30 \\
\hline P10 & $(2.03,5.13)$ & $0-2$ & Com vinculação exterior & 0.0 & Centro & 0.30 \\
\hline P11 & $(6.48,5.13)$ & $0-2$ & Com vinculação exterior & 0.0 & Centro & 0.30 \\
\hline P12 & $(11.38,5.13)$ & $0-2$ & Com vinculação exterior & 0.0 & Centro & 0.30 \\
\hline P13 & $(9.38,2.33)$ & $0-1$ & Com vinculação exterior & 0.0 & Centro & 0.30 \\
\hline P14 & $(2.03,0.68)$ & $0-2$ & Com vinculação exterior & 0.0 & Centro & 0.30 \\
\hline P15 & $(6.48,0.68)$ & $0-2$ & Com vinculação exterior & 0.0 & Centro & 0.30 \\
\hline P16 & $(11.38,0.68)$ & $0-2$ & Com vinculação exterior & 0.0 & Centro & 0.30 \\
\hline
\end{tabular}

\section{9.- DIMENSÕES, COEFICIENTES DE ENGASTAMENTO E COEFICIENTES DE FLAMBAGEM PARA CADA PISO}

\begin{tabular}{|c|c|c|c|c|c|c|c|}
\hline \multirow[t]{2}{*}{ Pilar } & \multirow{2}{*}{$\begin{array}{c}\text { Pis } \\
0\end{array}$} & \multirow{2}{*}{$\begin{array}{c}\text { Dimensõe } \\
\mathrm{s} \\
(\mathrm{cm})\end{array}$} & \multicolumn{2}{|c|}{$\begin{array}{l}\text { Coeficiente de } \\
\text { engastamento }\end{array}$} & \multicolumn{2}{|c|}{$\begin{array}{l}\text { Coeficiente de } \\
\text { flambagem }\end{array}$} & \multirow{2}{*}{$\begin{array}{c}\text { Coeficiente de rigidez } \\
\text { axial }\end{array}$} \\
\hline & & & Ext.Superior & Ext.Inferior & $x$ & $\mathrm{Y}$ & \\
\hline \multirow{2}{*}{$\begin{array}{l}\mathrm{P} 3, \mathrm{P} 1, \mathrm{P} 7, \mathrm{P} 10, \\
\mathrm{P} 14, \\
\mathrm{P} 16, \mathrm{P} 12, \mathrm{P} 6, \mathrm{P} 11, \\
\mathrm{P} 8, \mathrm{P} 2\end{array}$} & 2 & $20 \times 20$ & 0.30 & 1.00 & 1.00 & 1.00 & 2.00 \\
\hline & 1 & $20 \times 20$ & 1.00 & 1.00 & 1.00 & 1.00 & 2.00 \\
\hline \multirow{2}{*}{ P15 } & 2 & $25 \times 20$ & 0.30 & 1.00 & 1.00 & 1.00 & 2.00 \\
\hline & 1 & $25 \times 20$ & 1.00 & 1.00 & 1.00 & 1.00 & 2.00 \\
\hline \multirow{2}{*}{ P5 } & 2 & $55 \times 15$ & 0.30 & 1.00 & 1.00 & 1.00 & 2.00 \\
\hline & 1 & $55 \times 15$ & 1.00 & 1.00 & 1.00 & 1.00 & 2.00 \\
\hline $\mathrm{P} 4, \mathrm{P} 9, \mathrm{P} 13$ & 1 & $20 \times 20$ & 0.30 & 1.00 & 1.00 & 1.00 & 2.00 \\
\hline
\end{tabular}

\subsection{0.- RELATÓRIO DE PANOS}

Tipos de lajes consideradas

\begin{tabular}{|c|l|}
\hline Nome & \multicolumn{1}{|c|}{ Descrição } \\
\hline B12 & LAJE DE VIGOTAS DE CONCRETO \\
& Altura do bloco/molde: $8 \mathrm{~cm}$ \\
& Espessura camada de compressão: $4 \mathrm{~cm}$ \\
& Entre-eixos: $38 \mathrm{~cm}$ \\
Bloco/Molde: Cerâmica \\
Largura da nervura: $15 \mathrm{~cm}$ \\
Volume de concreto: $0.088 \mathrm{~m}^{3} / \mathrm{m}^{2}$ \\
Peso próprio: $2.042 \mathrm{kN} / \mathrm{m}^{2}$ \\
Incremento da largura da nervura: $2 \mathrm{~cm}$ \\
Verificação da flecha: Como vigota armada
\end{tabular}




\subsection{1.- LAJES E ELEMENTOS DE FUNDAÇÃO}

-Tensão admissível em combinações fundamentais: $0.245 \mathrm{MPa}$

-Tensão admissível em combinações acidentais: $0.368 \mathrm{MPa}$

\subsection{2.- MATERIAIS UTILIZADOS}

\subsection{1.- Concretos}

\begin{tabular}{|c|c|c|c|c|c|c|}
\hline \multirow[b]{2}{*}{ Elemento } & \multirow[b]{2}{*}{ Concreto } & \multirow{2}{*}{$\begin{array}{c}f_{\mathrm{ck}} \\
(\mathrm{MPa})\end{array}$} & \multirow[b]{2}{*}{$\gamma_{c}$} & \multicolumn{2}{|r|}{ Agregado } & \multirow{2}{*}{$\begin{array}{c}E_{\mathrm{c}} \\
(\mathrm{MPa})\end{array}$} \\
\hline & & & & Natureza & $\begin{array}{c}\text { Tamanho máximo } \\
(\mathrm{mm})\end{array}$ & \\
\hline Elementos de fundação & C30, con. desfav. & 30 & 1.54 & Granito & 15 & $2683 \varepsilon$ \\
\hline Pisos & C30, con.desfav. & 30 & 1.54 & Granito & 15 & $2683 \varepsilon$ \\
\hline Pilares e pilares-paredes & C30, con.desfav. & 30 & 1.54 & Granito & 15 & $2683 \varepsilon$ \\
\hline Cortinas & C20, em geral & 20 & 1.40 & Granito & 15 & 21287 \\
\hline
\end{tabular}

\subsection{2.- Aços por elemento}

6.12.2.1.- Aços em barras

\begin{tabular}{|l|l|c|c|}
\hline \multicolumn{1}{|c|}{ Elemento } & \multicolumn{1}{|c|}{ Aço } & $\begin{array}{c}\mathrm{f}_{\mathrm{yk}} \\
(\mathrm{MPa})\end{array}$ & $\gamma_{\mathrm{s}}$ \\
\hline Todos & CA-50 e CA-60 & $500 \mathrm{a} 600$ & 1.15 \\
\hline
\end{tabular}

6.12.2.2.- Aços em perfis

\begin{tabular}{|l|c|c|c|}
\hline Tipo de aço para perfis & Aço & $\begin{array}{c}\text { Limite elástico } \\
\text { (MPa) }\end{array}$ & $\begin{array}{c}\text { Módulo de elasticidade } \\
\text { (GPa) }\end{array}$ \\
\hline Aço dobrado & CF-26 & 260 & 200 \\
Aço laminado & A-36 & 250 & 200 \\
\hline
\end{tabular}

\subsection{3.- VERIFICAÇÃO DA FUNDAÇÃO}

Referência: P1

Dimensões: $90 \times 90 \times 30 / 20$

Soldados: Xi: Ø10c/28 Yi:Ø10c/28

\begin{tabular}{|l|l|l|}
\hline Verificação & Valores & Estado \\
\hline $\begin{array}{l}\text { Ângulo máximo talude: } \\
\text { Critério da CYPE Ingenieros }\end{array}$ & $\begin{array}{l}\text { Máximo: } 30 \text { graus } \\
\text { Calculado: } 18.4349 \text { graus }\end{array}$ & Passa \\
\hline $\begin{array}{l}\text { Tensões sobre o terreno: } \\
\text { Critério da CYPE Ingenieros }\end{array}$ & & \\
\hline
\end{tabular}




\begin{tabular}{|c|c|c|}
\hline $\begin{array}{l}\text { Referência: P1 } \\
\text { Dimensões: } 90 \text { × } 90 \text { × } 30 / 20 \\
\text { Soldados: Xi: Ø10c/28 Yi:Ø10c/28 }\end{array}$ & & \\
\hline Verificação & Valores & Estado \\
\hline $\begin{array}{l}\text {-Tensão média em combinações fundamentais: } \\
\text {-Tensão máxima em combinações fundamentais: }\end{array}$ & $\begin{array}{l}\text { Máximo: } 0.24525 \mathrm{MPa} \\
\text { Calculado: } 0.10173 \mathrm{MPa} \\
\text { Máximo: } 0.306563 \mathrm{MPa} \\
\text { Calculado: } 0.253098 \mathrm{MPa}\end{array}$ & $\begin{array}{l}\text { Passa } \\
\text { Passa }\end{array}$ \\
\hline $\begin{array}{l}\text { Tombamento da sapata: } \\
\text { Se o \% de reserva de segurança é maior que zero, pode ser dito que } \\
\text { os coeficientes de segurança ao tombamento são maiores que os } \\
\text { valores exatos exigidos para todas as combinaçoses de equilíbrio. } \\
\text { - Na direção X: } \\
\text { - Na direção Y: }\end{array}$ & $\begin{array}{l}\text { Reserva segurança: } 248.2 \% \\
\text { Reserva segurança: } 218.6 \%\end{array}$ & Passa \\
\hline $\begin{array}{l}\text { Flexão na sapata: } \\
\text { - Na direção } X: \\
\text { - Na direção Y: }\end{array}$ & $\begin{array}{l}\text { Momento: } 12.61 \mathrm{kN} \cdot \mathrm{m} \\
\text { Momento: } 13.03 \mathrm{kN} \cdot \mathrm{m}\end{array}$ & $\begin{array}{l}\text { Passa } \\
\text { Passa }\end{array}$ \\
\hline $\begin{array}{l}\text { Cortante na sapata: } \\
\text { - Na direção X: } \\
\text { - Na direção Y: }\end{array}$ & $\begin{array}{l}\text { Cortante: } 19.23 \mathrm{kN} \\
\text { Cortante: } 19.91 \mathrm{kN}\end{array}$ & $\begin{array}{l}\text { Passa } \\
\text { Passa }\end{array}$ \\
\hline $\begin{array}{l}\text { Compressão oblíqua na sapata: } \\
\text { - Combinações fundamentais: } \\
\text { Critério da CYPE Ingenieros }\end{array}$ & $\begin{array}{l}\text { Máximo: } 5844.1 \mathrm{kN} / \mathrm{m}^{2} \\
\text { Calculado: } 550 \mathrm{kN} / \mathrm{m}^{2}\end{array}$ & Passa \\
\hline $\begin{array}{l}\text { Altura mínima: } \\
\text { Critério da CYPE Ingenieros }\end{array}$ & $\begin{array}{l}\text { Mínimo: } 15 \mathrm{~cm} \\
\text { Calculado: } 20 \mathrm{~cm}\end{array}$ & Passa \\
\hline $\begin{array}{l}\text { Espaço para ancorar arranques na fundação: } \\
\text {-P1: }\end{array}$ & $\begin{array}{l}\text { Mínimo: } 12 \mathrm{~cm} \\
\text { Calculado: } 24 \mathrm{~cm}\end{array}$ & Passa \\
\hline $\begin{array}{l}\text { Quantidade geométrica mínima: } \\
\text { Critério da CYPE Ingenieros } \\
\text { - Armadura inferior direção X: } \\
\text { - Armadura inferior direção Y: }\end{array}$ & $\begin{array}{l}\text { Mínimo: } 0.001 \\
\text { Calculado: } 0.001 \\
\text { Calculado: } 0.001\end{array}$ & $\begin{array}{l}\text { Passa } \\
\text { Passa }\end{array}$ \\
\hline $\begin{array}{l}\text { Quantia mínima necessária por flexão: } \\
\text { Norma Brasileira ABNT NBR 6118:2014. Artigo 17.3.5.2 } \\
\text { - Armadura inferior direção X: } \\
\text { - Armadura inferior direção Y: }\end{array}$ & $\begin{array}{l}\text { Mínimo: } 0.0006 \\
\text { Calculado: } 0.0011 \\
\text { Calculado: } 0.0011\end{array}$ & $\begin{array}{l}\text { Passa } \\
\text { Passa }\end{array}$ \\
\hline $\begin{array}{l}\text { Diâmetro mínimo das barras: } \\
\text { - Malha inferior: } \\
\text { Critério da CYPE Ingenieros }\end{array}$ & $\begin{array}{l}\text { Mínimo: } 10 \mathrm{~mm} \\
\text { Calculado: } 10 \mathrm{~mm}\end{array}$ & Passa \\
\hline $\begin{array}{l}\text { Espaçamento máximo entre barras: } \\
\text { Critério da CYPE Ingenieros } \\
\text { - Armadura inferior direção X: } \\
\text { - Armadura inferior direção Y: }\end{array}$ & $\begin{array}{l}\text { Máximo: } 30 \mathrm{~cm} \\
\text { Calculado: } 28 \mathrm{~cm} \\
\text { Calculado: } 28 \mathrm{~cm}\end{array}$ & $\begin{array}{l}\text { Passa } \\
\text { Passa }\end{array}$ \\
\hline
\end{tabular}




\begin{tabular}{|c|c|c|}
\hline \multicolumn{3}{|l|}{$\begin{array}{l}\text { Referência: P1 } \\
\text { Dimensões: } 90 \text { × } 90 \text { × } 30 / 20 \\
\text { Soldados: Xi: } \varnothing 10 c / 28 \text { Yi: Ø10c/28 }\end{array}$} \\
\hline Verificação & Valores & Estado \\
\hline $\begin{array}{l}\text { Espaçamento mínimo entre barras: } \\
\text { Critério da CYPE Ingenieros, baseado em: J. Calavera. "Cálculo de } \\
\text { Estructuras de Cimentación". Capítulo 3.16 } \\
\text { - Armadura inferior direção X: } \\
\text { - Armadura inferior direção Y: }\end{array}$ & $\begin{array}{l}\text { Mínimo: } 10 \mathrm{~cm} \\
\text { Calculado: } 28 \mathrm{~cm} \\
\text { Calculado: } 28 \mathrm{~cm}\end{array}$ & $\begin{array}{l}\text { Passa } \\
\text { Passa }\end{array}$ \\
\hline $\begin{array}{l}\text { Comprimento de ancoragem: } \\
\text { Critério do livro "Ć́lculo de estructuras de cimentación", J. Calavera. } \\
\text { Ed. INTEMAC, } 1991 \\
\text { - Armadura inf. direção X para dir: } \\
\text { - Armadura inf. direção X para esq: } \\
\text { - Armadura inf. direção Y para cima: } \\
\text { - Armadura inf. direção Y para baixo: }\end{array}$ & $\begin{array}{l}\text { Calculado: } 32 \mathrm{~cm} \\
\text { Mínimo: } 11 \mathrm{~cm} \\
\text { Mínimo: } 22 \mathrm{~cm} \\
\text { Mínimo: } 22 \mathrm{~cm} \\
\text { Mínimo: } 11 \mathrm{~cm}\end{array}$ & $\begin{array}{l}\text { Passa } \\
\text { Passa } \\
\text { Passa } \\
\text { Passa }\end{array}$ \\
\hline $\begin{array}{l}\text { Comprimento mínimo das dobras: } \\
\text { - Armadura inf. direção X para dir: } \\
\text { - Armadura inf. direção X para esq: } \\
\text { - Armadura inf. direção Y para cima: } \\
\text { - Armadura inf. direção Y para baixo: }\end{array}$ & $\begin{array}{l}\text { Mínimo: } 11 \mathrm{~cm} \\
\text { Calculado: } 21 \mathrm{~cm} \\
\text { Calculado: } 21 \mathrm{~cm} \\
\text { Calculado: } 21 \mathrm{~cm} \\
\text { Calculado: } 21 \mathrm{~cm}\end{array}$ & $\begin{array}{l}\text { Passa } \\
\text { Passa } \\
\text { Passa } \\
\text { Passa }\end{array}$ \\
\hline $\begin{array}{l}\text { Todas as verificações foram } \\
\text { Referência: } \mathrm{P} 2 \\
\text { Dimensões: } 90 \times 90 \times 30 / 20 \\
\text { Soldados: } \mathrm{Xi}: \varnothing 10 \mathrm{c} / 28 \mathrm{Yi}: \varnothing 10 \mathrm{c} / 28\end{array}$ & cumpridas & \\
\hline Verificação & Valores & Estado \\
\hline $\begin{array}{l}\text { Ângulo máximo talude: } \\
\text { Critério da CYPE Ingenieros }\end{array}$ & $\begin{array}{l}\text { Máximo: } 30 \text { graus } \\
\text { Calculado: } 18.4349 \text { graus }\end{array}$ & Passa \\
\hline $\begin{array}{l}\text { Tensões sobre o terreno: } \\
\text { Critério da CYPE Ingenieros } \\
\text { - Tensão média em combinações fundamentais: } \\
\text { - Tensão máxima em combinações fundamentais: }\end{array}$ & $\begin{array}{l}\text { Máximo: } 0.24525 \mathrm{MPa} \\
\text { Calculado: } 0.132729 \mathrm{MPa} \\
\text { Máximo: } 0.306563 \mathrm{MPa} \\
\text { Calculado: } 0.196887 \mathrm{MPa}\end{array}$ & $\begin{array}{l}\text { Passa } \\
\text { Passa }\end{array}$ \\
\hline $\begin{array}{l}\text { Tombamento da sapata: } \\
\text { se o \% de reserva de segurança é maior que zero, pode ser dito que } \\
\text { os coeficientes de segurança ao tombamento são maiores que os } \\
\text { valores exatos exigidos para todas as combinaçôes de equilíbrio. } \\
\text { - Na direção X: } \\
\text { - Na direção Y: }\end{array}$ & $\begin{array}{l}\text { Reserva segurança: } 792.6 \% \\
\text { Reserva segurança: } 1291.9 \%\end{array}$ & $\begin{array}{l}\text { Passa } \\
\text { Passa }\end{array}$ \\
\hline $\begin{array}{l}\text { Flexão na sapata: } \\
\text { - Na direção X: } \\
\text { - Na direção Y: }\end{array}$ & $\begin{array}{l}\text { Momento: } 14.06 \mathrm{kN} \cdot \mathrm{m} \\
\text { Momento: } 13.18 \mathrm{kN} \cdot \mathrm{m}\end{array}$ & $\begin{array}{l}\text { Passa } \\
\text { Passa }\end{array}$ \\
\hline & & \\
\hline
\end{tabular}




\begin{tabular}{|c|c|c|}
\hline \multicolumn{3}{|l|}{$\begin{array}{l}\text { Referência: P2 } \\
\text { Dimensões: } 90 \text { × } 90 \text { × } 30 / 20 \\
\text { Soldados: Xi: Ø10c/28 Yi:Ø10c/28 }\end{array}$} \\
\hline Verificação & Valores & Estado \\
\hline $\begin{array}{l}\text { - Na direção } X: \\
\text { - Na direção Y: }\end{array}$ & $\begin{array}{l}\text { Cortante: } 20.31 \mathrm{kN} \\
\text { Cortante: } 18.84 \mathrm{kN}\end{array}$ & $\begin{array}{l}\text { Passa } \\
\text { Passa }\end{array}$ \\
\hline $\begin{array}{l}\text { Compressão oblíqua na sapata: } \\
\text { - Combinações fundamentais: } \\
\text { Critério da CYPE Ingenieros }\end{array}$ & $\begin{array}{l}\text { Máximo: } 5844.1 \mathrm{kN} / \mathrm{m}^{2} \\
\text { Calculado: } 825.8 \mathrm{kN} / \mathrm{m}^{2}\end{array}$ & Passa \\
\hline $\begin{array}{l}\text { Altura mínima: } \\
\text { Critério da CYPE Ingenieros }\end{array}$ & $\begin{array}{l}\text { Mínimo: } 15 \mathrm{~cm} \\
\text { Calculado: } 20 \mathrm{~cm}\end{array}$ & Passa \\
\hline $\begin{array}{l}\text { Espaço para ancorar arranques na fundação: } \\
\text { - P2: }\end{array}$ & $\begin{array}{l}\text { Mínimo: } 12 \mathrm{~cm} \\
\text { Calculado: } 24 \mathrm{~cm}\end{array}$ & Passa \\
\hline $\begin{array}{l}\text { Quantidade geométrica mínima: } \\
\text { Critério da CYPE Ingenieros } \\
\text { - Armadura inferior direção X: } \\
\text { - Armadura inferior direção Y: }\end{array}$ & $\begin{array}{l}\text { Mínimo: } 0.001 \\
\text { Calculado: } 0.001 \\
\text { Calculado: } 0.001\end{array}$ & $\begin{array}{l}\text { Passa } \\
\text { Passa }\end{array}$ \\
\hline $\begin{array}{l}\text { Quantia mínima necessária por flexão: } \\
\text { Norma Brasileira ABNT NBR 6118:2014. Artigo 17.3.5.2 } \\
\text { - Armadura inferior direção X: } \\
\text { - Armadura inferior direção Y: }\end{array}$ & $\begin{array}{l}\text { Calculado: } 0.0011 \\
\text { Mínimo: } 0.0007 \\
\text { Mínimo: } 0.0006\end{array}$ & $\begin{array}{l}\text { Passa } \\
\text { Passa }\end{array}$ \\
\hline $\begin{array}{l}\text { Diâmetro mínimo das barras: } \\
\text { - Malha inferior: } \\
\text { Critério da CYPE Ingenieros }\end{array}$ & $\begin{array}{l}\text { Mínimo: } 10 \mathrm{~mm} \\
\text { Calculado: } 10 \mathrm{~mm}\end{array}$ & Passa \\
\hline $\begin{array}{l}\text { Espaçamento máximo entre barras: } \\
\text { Critério da CYPE Ingenieros } \\
\text { - Armadura inferior direção } \mathrm{X} \text { : } \\
\text { - Armadura inferior direção } \mathrm{Y} \text { : }\end{array}$ & $\begin{array}{l}\text { Máximo: } 30 \mathrm{~cm} \\
\text { Calculado: } 28 \mathrm{~cm} \\
\text { Calculado: } 28 \mathrm{~cm}\end{array}$ & $\begin{array}{l}\text { Passa } \\
\text { Passa }\end{array}$ \\
\hline $\begin{array}{l}\text { Espaçamento mínimo entre barras: } \\
\text { Critério da CYPE Ingenieros, baseado em: J. Calavera. "Cálculo de } \\
\text { Estructuras de Cimentación". Capítulo 3.16 } \\
\text { - Armadura inferior direção X: } \\
\text { - Armadura inferior direção Y: }\end{array}$ & $\begin{array}{l}\text { Mínimo: } 10 \mathrm{~cm} \\
\text { Calculado: } 28 \mathrm{~cm} \\
\text { Calculado: } 28 \mathrm{~cm}\end{array}$ & $\begin{array}{l}\text { Passa } \\
\text { Passa }\end{array}$ \\
\hline $\begin{array}{l}\text { Comprimento de ancoragem: } \\
\text { Critério do livro "Célculo de estructuras de cimentación", J. Calavera. } \\
\text { Ed. INTEMAC, } 1991 \\
\text { - Armadura inf. direção X para dir: } \\
\text { - Armadura inf. direção X para esq: } \\
\text { - Armadura inf. direção Y para cima: } \\
\text { - Armadura inf. direção Y para baixo: }\end{array}$ & $\begin{array}{l}\text { Mínimo: } 22 \mathrm{~cm} \\
\text { Calculado: } 32 \mathrm{~cm} \\
\text { Calculado: } 32 \mathrm{~cm} \\
\text { Calculado: } 32 \mathrm{~cm} \\
\text { Calculado: } 32 \mathrm{~cm}\end{array}$ & $\begin{array}{l}\text { Passa } \\
\text { Passa } \\
\text { Passa } \\
\text { Passa }\end{array}$ \\
\hline Comprimento mínimo das dobras: & Mínimo: $11 \mathrm{~cm}$ & \\
\hline
\end{tabular}




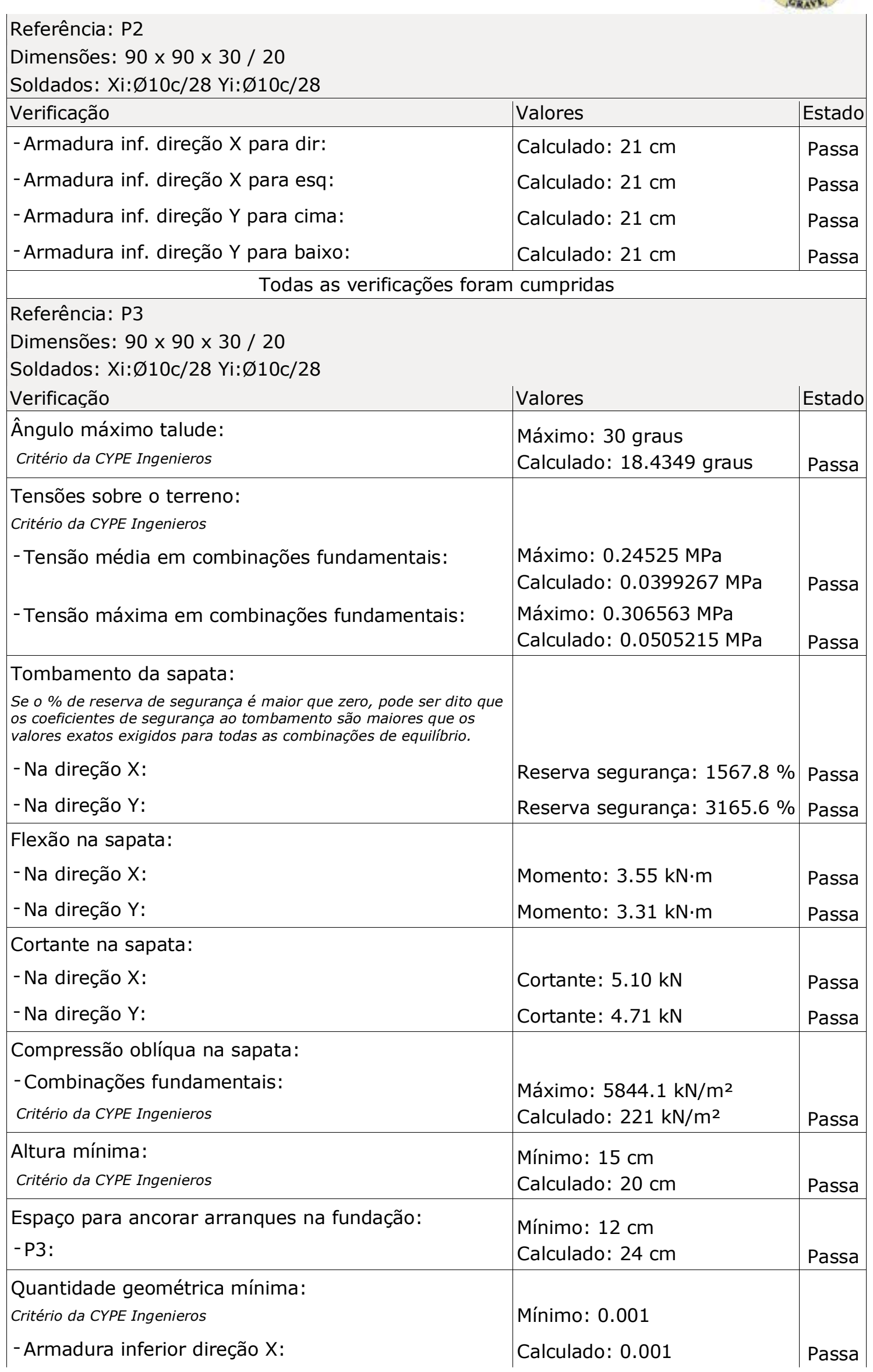




\begin{tabular}{|c|c|c|}
\hline \\
\hline $\begin{array}{l}\text { Referência: P3 } \\
\text { Dimensões: } 90 \text { × } 90 \text { × } 30 / 20 \\
\text { Soldados: Xi: } \varnothing 10 c / 28 \text { Yi: Ø10c/28 }\end{array}$ & & \\
\hline Verificação & Valores & Estado \\
\hline - Armadura inferior direção Y: & Calculado: 0.001 & Passa \\
\hline $\begin{array}{l}\text { Quantia mínima necessária por flexão: } \\
\text { Norma Brasileira ABNT NBR 6118:2014. Artigo 17.3.5.2 } \\
\text { - Armadura inferior direção X: } \\
\text { - Armadura inferior direção Y: }\end{array}$ & $\begin{array}{l}\text { Mínimo: } 0.0002 \\
\text { Calculado: } 0.0011 \\
\text { Calculado: } 0.0011\end{array}$ & $\begin{array}{l}\text { Passa } \\
\text { Passa }\end{array}$ \\
\hline $\begin{array}{l}\text { Diâmetro mínimo das barras: } \\
\text { - Malha inferior: } \\
\text { Critério da CYPE Ingenieros }\end{array}$ & $\begin{array}{l}\text { Mínimo: } 10 \mathrm{~mm} \\
\text { Calculado: } 10 \mathrm{~mm}\end{array}$ & Passa \\
\hline $\begin{array}{l}\text { Espaçamento máximo entre barras: } \\
\text { Critério da CYPE Ingenieros } \\
\text { - Armadura inferior direção X: } \\
\text { - Armadura inferior direção Y: }\end{array}$ & $\begin{array}{l}\text { Máximo: } 30 \mathrm{~cm} \\
\text { Calculado: } 28 \mathrm{~cm} \\
\text { Calculado: } 28 \mathrm{~cm}\end{array}$ & $\begin{array}{l}\text { Passa } \\
\text { Passa }\end{array}$ \\
\hline $\begin{array}{l}\text { Espaçamento mínimo entre barras: } \\
\text { Critério da CYPE Ingenieros, baseado em: J. Calavera. "Cálculo de } \\
\text { Estructuras de Cimentación". Capítulo 3.16 } \\
\text { - Armadura inferior direção X: } \\
\text { - Armadura inferior direção Y: }\end{array}$ & $\begin{array}{l}\text { Mínimo: } 10 \mathrm{~cm} \\
\text { Calculado: } 28 \mathrm{~cm} \\
\text { Calculado: } 28 \mathrm{~cm}\end{array}$ & $\begin{array}{l}\text { Passa } \\
\text { Passa }\end{array}$ \\
\hline $\begin{array}{l}\text { Comprimento de ancoragem: } \\
\text { Critério do livro "Cálculo de estructuras de cimentación", J. Calavera. } \\
\text { Ed. INTEMAC, } 1991 \\
\text { - Armadura inf. direção X para dir: } \\
\text { - Armadura inf. direção X para esq: } \\
\text { - Armadura inf. direção Y para cima: } \\
\text { - Armadura inf. direção Y para baixo: }\end{array}$ & $\begin{array}{l}\text { Mínimo: } 11 \mathrm{~cm} \\
\text { Calculado: } 11 \mathrm{~cm} \\
\text { Calculado: } 11 \mathrm{~cm} \\
\text { Calculado: } 11 \mathrm{~cm} \\
\text { Calculado: } 11 \mathrm{~cm}\end{array}$ & $\begin{array}{l}\text { Passa } \\
\text { Passa } \\
\text { Passa } \\
\text { Passa }\end{array}$ \\
\hline $\begin{array}{l}\text { Todas as verificações fo } \\
\text { Referência: } \mathrm{P} 4 \\
\text { Dimensões: } 90 \times 90 \times 30 / 20 \\
\text { Soldados: Xi: Ø10c/28 Yi:Ø10c/28 }\end{array}$ & cumpridas & \\
\hline Verificação & Valores & Estado \\
\hline $\begin{array}{l}\text { Ângulo máximo talude: } \\
\text { Critério da CYPE Ingenieros }\end{array}$ & $\begin{array}{l}\text { Máximo: } 30 \text { graus } \\
\text { Calculado: } 18.4349 \text { graus }\end{array}$ & Passa \\
\hline $\begin{array}{l}\text { Tensões sobre o terreno: } \\
\text { Critério da CYPE Ingenieros } \\
\text { - Tensão média em combinações fundamentais: } \\
\text { - Tensão máxima em combinações fundamentais: }\end{array}$ & $\begin{array}{l}\text { Máximo: } 0.24525 \mathrm{MPa} \\
\text { Calculado: } 0.0626859 \mathrm{MPa} \\
\text { Máximo: } 0.306563 \mathrm{MPa} \\
\text { Calculado: } 0.170988 \mathrm{MPa}\end{array}$ & Passa \\
\hline
\end{tabular}




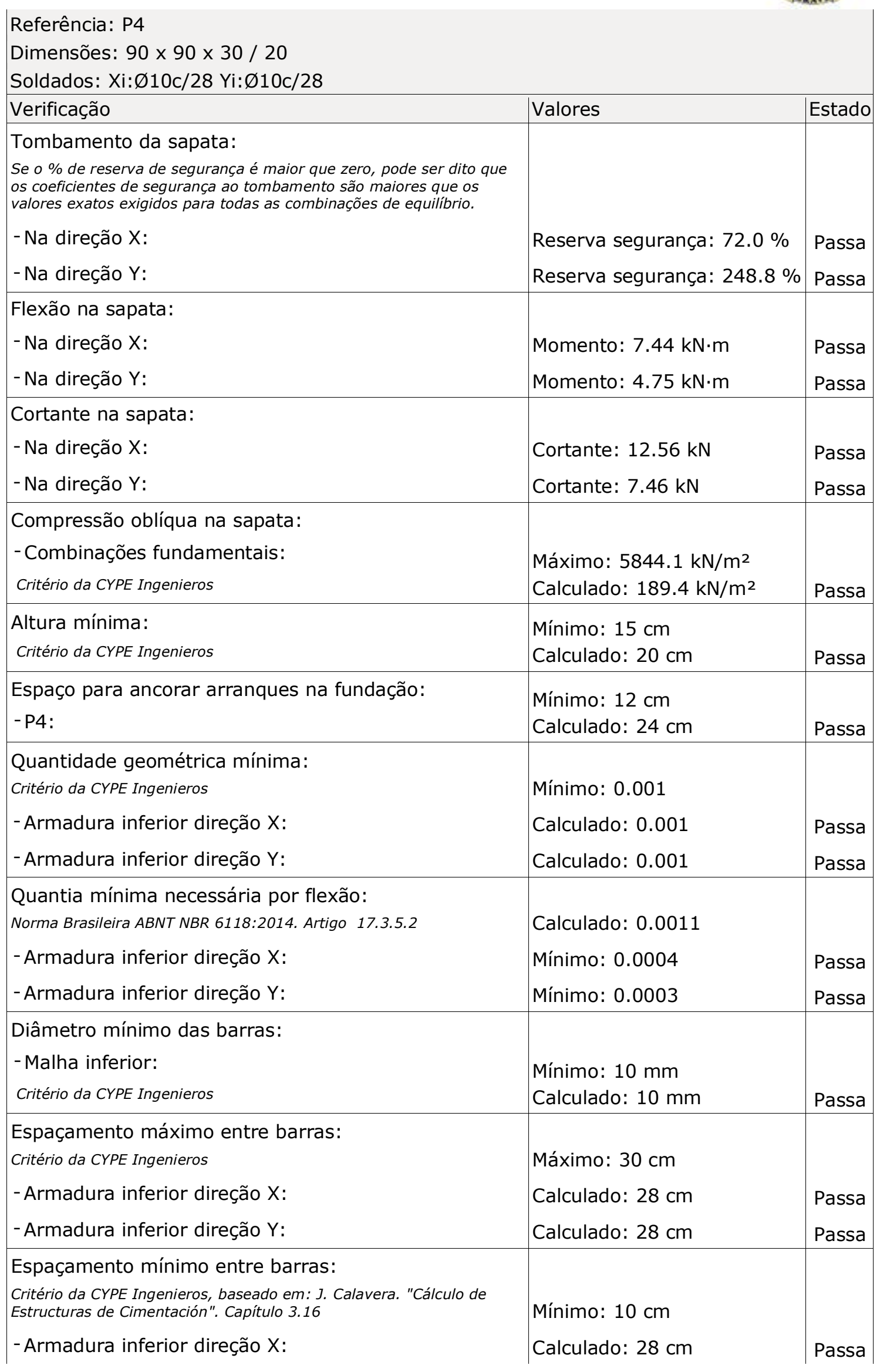




\begin{tabular}{|c|c|c|}
\hline \\
\hline $\begin{array}{l}\text { Referência: P4 } \\
\text { Dimensões: } 90 \text { x } 90 \text { x } 30 / 20 \\
\text { Soldados: Xi: Ø10c/28 Yi: Ø10c/28 }\end{array}$ & & \\
\hline Verificação & Valores & Estado \\
\hline - Armadura inferior direção Y: & Calculado: $28 \mathrm{~cm}$ & Passa \\
\hline $\begin{array}{l}\text { Comprimento de ancoragem: } \\
\text { Critério do livro "Cálculo de estructuras de cimentación", J. Calavera. } \\
\text { Ed. INTEMAC, } 1991 \\
\text { - Armadura inf. direção X para dir: } \\
\text { - Armadura inf. direção X para esq: } \\
\text { - Armadura inf. direção Y para cima: } \\
\text { - Armadura inf. direção Y para baixo: }\end{array}$ & $\begin{array}{l}\text { Mínimo: } 22 \mathrm{~cm} \\
\text { Calculado: } 32 \mathrm{~cm} \\
\text { Mínimo: } 11 \mathrm{~cm} \\
\text { Calculado: } 32 \mathrm{~cm} \\
\text { Mínimo: } 11 \mathrm{~cm} \\
\text { Calculado: } 11 \mathrm{~cm} \\
\text { Mínimo: } 11 \mathrm{~cm} \\
\text { Calculado: } 11 \mathrm{~cm}\end{array}$ & $\begin{array}{l}\text { Passa } \\
\text { Passa } \\
\text { Passa } \\
\text { Passa }\end{array}$ \\
\hline $\begin{array}{l}\text { Comprimento mínimo das dobras: } \\
\text { - Armadura inf. direção X para dir: } \\
\text { - Armadura inf. direção X para esq: }\end{array}$ & $\begin{array}{l}\text { Mínimo: } 11 \mathrm{~cm} \\
\text { Calculado: } 21 \mathrm{~cm} \\
\text { Calculado: } 21 \mathrm{~cm}\end{array}$ & $\begin{array}{l}\text { Passa } \\
\text { Passa }\end{array}$ \\
\hline Todas as verificações foran & cumpridas & \\
\hline $\begin{array}{l}\text { Referência: P5 } \\
\text { Dimensões: } 125 \text { x } 125 \text { x } 40 \text { / } 30 \\
\text { Soldados: Xi: Ø10c/20 Yi:Ø10c/20 }\end{array}$ & & \\
\hline Verificação & Valores & Estado \\
\hline $\begin{array}{l}\text { Ângulo máximo talude: } \\
\text { Critério da CYPE Ingenieros }\end{array}$ & $\begin{array}{l}\text { Máximo: } 30 \text { graus } \\
\text { Calculado: } 18.4349 \text { graus }\end{array}$ & Passa \\
\hline $\begin{array}{l}\text { Tensões sobre o terreno: } \\
\text { Critério da CYPE Ingenieros } \\
\text { - Tensão média em combinações fundamentais: } \\
\text {-Tensão máxima em combinações fundamentais: }\end{array}$ & $\begin{array}{l}\text { Máximo: } 0.24525 \mathrm{MPa} \\
\text { Calculado: } 0.0802458 \mathrm{MPa} \\
\text { Máximo: } 0.306563 \mathrm{MPa} \\
\text { Calculado: } 0.125862 \mathrm{MPa}\end{array}$ & $\begin{array}{l}\text { Passa } \\
\text { Passa }\end{array}$ \\
\hline $\begin{array}{l}\text { Tombamento da sapata: } \\
\text { Se o \% de reserva de segurança é maior que zero, pode ser dito que } \\
\text { os coeficientes de segurança ao tombamento são maiores que os } \\
\text { valores exatos exigidos para todas as combinações de equilíbrio. } \\
\text { - Na direção X: } \\
\text { - Na direção Y: }\end{array}$ & $\begin{array}{l}\text { Reserva segurança: } 387.0 \% \\
\text { Reserva segurança: } 3963.0 \%\end{array}$ & $\begin{array}{l}\text { Passa } \\
\text { Passa }\end{array}$ \\
\hline $\begin{array}{l}\text { Flexão na sapata: } \\
\text { - Na direção } X: \\
\text { - Na direção } Y:\end{array}$ & $\begin{array}{l}\text { Momento: } 16.76 \mathrm{kN} \cdot \mathrm{m} \\
\text { Momento: } 21.58 \mathrm{kN} \cdot \mathrm{m}\end{array}$ & $\begin{array}{l}\text { Passa } \\
\text { Passa }\end{array}$ \\
\hline $\begin{array}{l}\text { Cortante na sapata: } \\
\text { - Na direção X: } \\
\text { - Na direção Y: }\end{array}$ & $\begin{array}{l}\text { Cortante: } 0.00 \mathrm{kN} \\
\text { Cortante: } 26.68 \mathrm{kN}\end{array}$ & $\begin{array}{l}\text { Passa } \\
\text { Passa }\end{array}$ \\
\hline
\end{tabular}




\begin{tabular}{|c|c|c|}
\hline \multicolumn{3}{|l|}{$\begin{array}{l}\text { Referência: P5 } \\
\text { Dimensões: } 125 \times 125 \times 40 / 30 \\
\text { Soldados: Xi: Ø10c/20 Yi: Ø10c/20 }\end{array}$} \\
\hline Verificação & Valores & Estado \\
\hline $\begin{array}{l}\text { Compressão oblíqua na sapata: } \\
\text { - Combinações fundamentais: } \\
\text { Critério da CYPE Ingenieros }\end{array}$ & $\begin{array}{l}\text { Máximo: } 5844.1 \mathrm{kN} / \mathrm{m}^{2} \\
\text { Calculado: } 366.9 \mathrm{kN} / \mathrm{m}^{2}\end{array}$ & Passa \\
\hline $\begin{array}{l}\text { Altura mínima: } \\
\text { Critério da CYPE Ingenieros }\end{array}$ & $\begin{array}{l}\text { Mínimo: } 15 \mathrm{~cm} \\
\text { Calculado: } 30 \mathrm{~cm}\end{array}$ & Passa \\
\hline $\begin{array}{l}\text { Espaço para ancorar arranques na fundação: } \\
\text { - P5: }\end{array}$ & $\begin{array}{l}\text { Mínimo: } 12 \mathrm{~cm} \\
\text { Calculado: } 34 \mathrm{~cm}\end{array}$ & Passa \\
\hline $\begin{array}{l}\text { Quantidade geométrica mínima: } \\
\text { Critério da CYPE Ingenieros } \\
\text { - Armadura inferior direção X: } \\
\text { - Armadura inferior direção Y: }\end{array}$ & $\begin{array}{l}\text { Mínimo: } 0.001 \\
\text { Calculado: } 0.001 \\
\text { Calculado: } 0.001\end{array}$ & $\begin{array}{l}\text { Passa } \\
\text { Passa }\end{array}$ \\
\hline $\begin{array}{l}\text { Quantia mínima necessária por flexão: } \\
\text { Norma Brasileira ABNT NBR 6118:2014. Artigo 17.3.5.2 } \\
\text { - Armadura inferior direção X: } \\
\text { - Armadura inferior direção Y: }\end{array}$ & $\begin{array}{l}\text { Calculado: } 0.0011 \\
\text { Mínimo: } 0.0003 \\
\text { Mínimo: } 0.0004\end{array}$ & $\begin{array}{l}\text { Passa } \\
\text { Passa }\end{array}$ \\
\hline $\begin{array}{l}\text { Diâmetro mínimo das barras: } \\
\text { - Malha inferior: } \\
\text { Critério da CYPE Ingenieros }\end{array}$ & $\begin{array}{l}\text { Mínimo: } 10 \mathrm{~mm} \\
\text { Calculado: } 10 \mathrm{~mm}\end{array}$ & Passa \\
\hline $\begin{array}{l}\text { Espaçamento máximo entre barras: } \\
\text { Critério da CYPE Ingenieros } \\
\text { - Armadura inferior direção X: } \\
\text { - Armadura inferior direção Y: }\end{array}$ & $\begin{array}{l}\text { Máximo: } 30 \mathrm{~cm} \\
\text { Calculado: } 20 \mathrm{~cm} \\
\text { Calculado: } 20 \mathrm{~cm}\end{array}$ & $\begin{array}{l}\text { Passa } \\
\text { Passa }\end{array}$ \\
\hline $\begin{array}{l}\text { Espaçamento mínimo entre barras: } \\
\text { Critério da CYPE Ingenieros, baseado em: J. Calavera. "Cálculo de } \\
\text { Estructuras de Cimentación". Capítulo 3.16 } \\
\text { - Armadura inferior direção X: } \\
\text { - Armadura inferior direção Y: }\end{array}$ & $\begin{array}{l}\text { Mínimo: } 10 \mathrm{~cm} \\
\text { Calculado: } 20 \mathrm{~cm} \\
\text { Calculado: } 20 \mathrm{~cm}\end{array}$ & $\begin{array}{l}\text { Passa } \\
\text { Passa }\end{array}$ \\
\hline $\begin{array}{l}\text { Comprimento de ancoragem: } \\
\text { Critério do livro "Cálculo de estructuras de cimentación", J. Calavera. } \\
\text { Ed. INTEMAC, } 1991\end{array}$ & & \\
\hline $\begin{array}{l}\text {-Armadura inf. direção X para dir: } \\
\text {-Armadura inf. direção X para esq: }\end{array}$ & $\begin{array}{l}\text { Mínimo: } 19 \mathrm{~cm} \\
\text { Calculado: } 19 \mathrm{~cm} \\
\text { Mínimo: } 19 \mathrm{~cm} \\
\text { Calculado: } 19 \mathrm{~cm}\end{array}$ & Passa \\
\hline $\begin{array}{l}\text { - Armadura inf. direção Y para cima: } \\
\text {-Armadura inf. direção Y para baixo: }\end{array}$ & $\begin{array}{l}\text { Mínimo: } 11 \mathrm{~cm} \\
\text { Calculado: } 22 \mathrm{~cm} \\
\text { Mínimo: } 11 \mathrm{~cm} \\
\text { Calculado: } 22 \mathrm{~cm}\end{array}$ & $\begin{array}{l}\text { Passa } \\
\text { Passa }\end{array}$ \\
\hline Comprimento mínimo das dobras: & Mínimo: $11 \mathrm{~cm}$ & \\
\hline
\end{tabular}




\begin{tabular}{|c|c|c|}
\hline \\
\hline $\begin{array}{l}\text { Referência: P5 } \\
\text { Dimensões: } 125 \times 125 \text { x } 40 \text { / } 30 \\
\text { Soldados: Xi: Ø10c/20 Yi:Ø10c/20 }\end{array}$ & & \\
\hline Verificação & Valores & Estado \\
\hline - Armadura inf. direção X para dir: & Calculado: $11 \mathrm{~cm}$ & Passa \\
\hline - Armadura inf. direção $X$ para esq: & Calculado: $11 \mathrm{~cm}$ & Passa \\
\hline \multicolumn{3}{|c|}{ Todas as verificações foram cumpridas } \\
\hline \multicolumn{3}{|c|}{$\begin{array}{l}\text { Referência: P6 } \\
\text { Dimensões: } 90 \text { × } 90 \text { x } 30 / 20 \\
\text { Soldados: Xi: Ø10c/28 Yi: Ø10c/28 }\end{array}$} \\
\hline Verificação & Valores & Estado \\
\hline $\begin{array}{l}\text { Ângulo máximo talude: } \\
\text { Critério da CYPE Ingenieros }\end{array}$ & $\begin{array}{l}\text { Máximo: } 30 \text { graus } \\
\text { Calculado: } 18.4349 \text { graus }\end{array}$ & Passa \\
\hline $\begin{array}{l}\text { Tensões sobre o terreno: } \\
\text { Critério da CYPE Ingenieros }\end{array}$ & & \\
\hline - Tensão média em combinações fundamentais: & $\begin{array}{l}\text { Máximo: } 0.24525 \mathrm{MPa} \\
\text { Calculado: } 0.0972171 \mathrm{MPa}\end{array}$ & Passa \\
\hline -Tensão máxima em combinações fundamentais: & $\begin{array}{l}\text { Máximo: } 0.306563 \mathrm{MPa} \\
\text { Calculado: } 0.167457 \mathrm{MPa}\end{array}$ & Passa \\
\hline Tombamento da sapata: & & \\
\hline $\begin{array}{l}\text { Se o \% de reserva de segurança é maior que zero, pode ser dito que } \\
\text { os coeficientes de segurança ao tombamento são maiores que os } \\
\text { valores exatos exigidos para todas as combinaçôes de equilíbrio. }\end{array}$ & & \\
\hline - Na direção X: & Reserva segurança: $380.3 \%$ & Passa \\
\hline - Na direção Y: & Reserva segurança: $1062.8 \%$ & Passa \\
\hline Flexão na sapata: & & \\
\hline - Na direção X: & Momento: $11.48 \mathrm{kN} \cdot \mathrm{m}$ & Passa \\
\hline - Na direção Y: & Momento: $9.70 \mathrm{kN} \cdot \mathrm{m}$ & Passa \\
\hline Cortante na sapata: & & \\
\hline - Na direção X: & Cortante: 16.97 kN & Passa \\
\hline - Na direção Y: & Cortante: $13.93 \mathrm{kN}$ & Passa \\
\hline $\begin{array}{l}\text { Compressão oblíqua na sapata: } \\
\text { - Combinações fundamentais: } \\
\text { Critério da CYPE Ingenieros }\end{array}$ & $\begin{array}{l}\text { Máximo: } 5844.1 \mathrm{kN} / \mathrm{m}^{2} \\
\text { Calculado: } 594.2 \mathrm{kN} / \mathrm{m}^{2}\end{array}$ & Passa \\
\hline $\begin{array}{l}\text { Altura mínima: } \\
\text { Critério da CYPE Ingenieros }\end{array}$ & $\begin{array}{l}\text { Mínimo: } 15 \mathrm{~cm} \\
\text { Calculado: } 20 \mathrm{~cm}\end{array}$ & Passa \\
\hline $\begin{array}{l}\text { Espaço para ancorar arranques na fundação: } \\
\text { - P6: }\end{array}$ & $\begin{array}{l}\text { Mínimo: } 12 \mathrm{~cm} \\
\text { Calculado: } 24 \mathrm{~cm}\end{array}$ & Passa \\
\hline $\begin{array}{l}\text { Quantidade geométrica mínima: } \\
\text { Critério da CYPE Ingenieros }\end{array}$ & Mínimo: 0.001 & \\
\hline - Armadura inferior direção X: & Calculado: 0.001 & Passa \\
\hline - Armadura inferior direção Y: & Calculado: 0.001 & Passa \\
\hline
\end{tabular}




\begin{tabular}{|c|c|c|}
\hline \multicolumn{3}{|l|}{$\begin{array}{l}\text { Referência: } \mathrm{P6} \\
\text { Dimensões: } 90 \text { × } 90 \text { × } 30 / 20 \\
\text { Soldados: } \mathrm{Xi}: \varnothing 10 \mathrm{c} / 28 \text { Yi: } \varnothing 10 \mathrm{c} / 28\end{array}$} \\
\hline Verificação & Valores & Estado \\
\hline $\begin{array}{l}\text { Quantia mínima necessária por flexão: } \\
\text { Norma Brasileira ABNT NBR 6118:2014. Artigo 17.3.5.2 } \\
\text { - Armadura inferior direção X: } \\
\text { - Armadura inferior direção Y: }\end{array}$ & $\begin{array}{l}\text { Calculado: } 0.0011 \\
\text { Mínimo: } 0.0006 \\
\text { Mínimo: } 0.0005\end{array}$ & $\begin{array}{l}\text { Passa } \\
\text { Passa }\end{array}$ \\
\hline $\begin{array}{l}\text { Diâmetro mínimo das barras: } \\
\text { - Malha inferior: } \\
\text { Critério da CYPE Ingenieros }\end{array}$ & $\begin{array}{l}\text { Mínimo: } 10 \mathrm{~mm} \\
\text { Calculado: } 10 \mathrm{~mm}\end{array}$ & Passa \\
\hline $\begin{array}{l}\text { Espaçamento máximo entre barras: } \\
\text { Critério da CYPE Ingenieros } \\
\text { - Armadura inferior direção X: } \\
\text { - Armadura inferior direção Y: }\end{array}$ & $\begin{array}{l}\text { Máximo: } 30 \mathrm{~cm} \\
\text { Calculado: } 28 \mathrm{~cm} \\
\text { Calculado: } 28 \mathrm{~cm}\end{array}$ & $\begin{array}{l}\text { Passa } \\
\text { Passa }\end{array}$ \\
\hline $\begin{array}{l}\text { Espaçamento mínimo entre barras: } \\
\text { Critério da CYPE Ingenieros, baseado em: J. Calavera. "Cálculo de } \\
\text { Estructuras de Cimentación". Capítulo 3.16 } \\
\text { - Armadura inferior direção X: } \\
\text { - Armadura inferior direção Y: }\end{array}$ & $\begin{array}{l}\text { Mínimo: } 10 \mathrm{~cm} \\
\text { Calculado: } 28 \mathrm{~cm} \\
\text { Calculado: } 28 \mathrm{~cm}\end{array}$ & $\begin{array}{l}\text { Passa } \\
\text { Passa }\end{array}$ \\
\hline $\begin{array}{l}\text { Comprimento de ancoragem: } \\
\text { Critério do livro "Cálculo de estructuras de cimentación", J. Calavera. } \\
\text { Ed. INTEMAC, } 1991 \\
\text { - Armadura inf. direção X para dir: } \\
\text { - Armadura inf. direção X para esq: } \\
\text { - Armadura inf. direção Y para cima: } \\
\text { - Armadura inf. direção Y para baixo: }\end{array}$ & $\begin{array}{l}\text { Calculado: } 32 \mathrm{~cm} \\
\text { Mínimo: } 22 \mathrm{~cm} \\
\text { Mínimo: } 11 \mathrm{~cm} \\
\text { Mínimo: } 22 \mathrm{~cm} \\
\text { Mínimo: } 11 \mathrm{~cm}\end{array}$ & $\begin{array}{l}\text { Passa } \\
\text { Passa } \\
\text { Passa } \\
\text { Passa }\end{array}$ \\
\hline $\begin{array}{l}\text { Comprimento mínimo das dobras: } \\
\text { - Armadura inf. direção X para dir: } \\
\text { - Armadura inf. direção X para esq: } \\
\text { - Armadura inf. direção Y para cima: } \\
\text { - Armadura inf. direção Y para baixo: }\end{array}$ & $\begin{array}{l}\text { Mínimo: } 11 \mathrm{~cm} \\
\text { Calculado: } 21 \mathrm{~cm} \\
\text { Calculado: } 21 \mathrm{~cm} \\
\text { Calculado: } 21 \mathrm{~cm} \\
\text { Calculado: } 21 \mathrm{~cm}\end{array}$ & $\begin{array}{l}\text { Passa } \\
\text { Passa } \\
\text { Passa } \\
\text { Passa }\end{array}$ \\
\hline Todas as verificações fora & cumpridas & \\
\hline $\begin{array}{l}\text { Referência: P7 } \\
\text { Dimensões: } 90 \text { × } 90 \text { x } 30 / 20 \\
\text { Soldados: Xi: Ø10c/28 Yi:Ø10c/28 } \\
\text { Verificação }\end{array}$ & Valores & Estado \\
\hline $\begin{array}{l}\text { Ângulo máximo talude: } \\
\text { Critério da CYPE Ingenieros }\end{array}$ & $\begin{array}{l}\text { Máximo: } 30 \text { graus } \\
\text { Calculado: } 18.4349 \text { graus }\end{array}$ & Passa \\
\hline $\begin{array}{l}\text { Tensões sobre o terreno: } \\
\text { Critério da CYPE Ingenieros } \\
\text { - Tensão média em combinações fundamentais: }\end{array}$ & $\begin{array}{l}\text { Máximo: } 0.24525 \mathrm{MPa} \\
\text { Calculado: } 0.108989 \mathrm{MPa}\end{array}$ & Passa \\
\hline
\end{tabular}




\begin{tabular}{|c|c|c|}
\hline $\begin{array}{l}\text { Referência: P7 } \\
\text { Dimensões: } 90 \text { x } 90 \text { x } 30 / 20 \\
\text { Soldados: Xi: Ø10c/28 Yi:Ø10c/28 }\end{array}$ & & \\
\hline Verificação & Valores & Estado \\
\hline -Tensão máxima em combinações fundamentais: & $\begin{array}{l}\text { Máximo: } 0.306563 \mathrm{MPa} \\
\text { Calculado: } 0.207972 \mathrm{MPa}\end{array}$ & Passa \\
\hline $\begin{array}{l}\text { Tombamento da sapata: } \\
\text { Se o \% de reserva de segurança é maior que zero, pode ser dito que } \\
\text { os coeficientes de segurança ao tombamento são maiores que os } \\
\text { valores exatos exigidos para todas as combinações de equilibrio. } \\
\text { - Na direção } \mathrm{X} \text { : } \\
\text { - Na direção Y: }\end{array}$ & $\begin{array}{l}\text { Reserva segurança: } 314.3 \% \\
\text { Reserva segurança: } 803.0 \%\end{array}$ & $\begin{array}{l}\text { Passa } \\
\text { Passa }\end{array}$ \\
\hline $\begin{array}{l}\text { Flexão na sapata: } \\
\text { - Na direção } X: \\
\text { - Na direção Y: }\end{array}$ & $\begin{array}{l}\text { Momento: } 13.73 \mathrm{kN} \cdot \mathrm{m} \\
\text { Momento: } 11.47 \mathrm{kN} \cdot \mathrm{m}\end{array}$ & $\begin{array}{l}\text { Passa } \\
\text { Passa }\end{array}$ \\
\hline $\begin{array}{l}\text { Cortante na sapata: } \\
\text { - Na direção X: } \\
\text { - Na direção Y: }\end{array}$ & $\begin{array}{l}\text { Cortante: } 20.50 \mathrm{kN} \\
\text { Cortante: } 16.58 \mathrm{kN}\end{array}$ & $\begin{array}{l}\text { Passa } \\
\text { Passa }\end{array}$ \\
\hline $\begin{array}{l}\text { Compressão oblíqua na sapata: } \\
\text { - Combinações fundamentais: } \\
\text { Critério da CYPE Ingenieros }\end{array}$ & $\begin{array}{l}\text { Máximo: } 5844.1 \mathrm{kN} / \mathrm{m}^{2} \\
\text { Calculado: } 671.1 \mathrm{kN} / \mathrm{m}^{2}\end{array}$ & Passa \\
\hline $\begin{array}{l}\text { Altura mínima: } \\
\text { Critério da CYPE Ingenieros }\end{array}$ & $\begin{array}{l}\text { Mínimo: } 15 \mathrm{~cm} \\
\text { Calculado: } 20 \mathrm{~cm}\end{array}$ & Passa \\
\hline $\begin{array}{l}\text { Espaço para ancorar arranques na fundação: } \\
\text { - P7: }\end{array}$ & $\begin{array}{l}\text { Mínimo: } 12 \mathrm{~cm} \\
\text { Calculado: } 24 \mathrm{~cm}\end{array}$ & Passa \\
\hline $\begin{array}{l}\text { Quantidade geométrica mínima: } \\
\text { Critério da CYPE Ingenieros } \\
\text { - Armadura inferior direção X: } \\
\text { - Armadura inferior direção Y: }\end{array}$ & $\begin{array}{l}\text { Mínimo: } 0.001 \\
\text { Calculado: } 0.001 \\
\text { Calculado: } 0.001\end{array}$ & $\begin{array}{l}\text { Passa } \\
\text { Passa }\end{array}$ \\
\hline $\begin{array}{l}\text { Quantia mínima necessária por flexão: } \\
\text { Norma Brasileira ABNT NBR 6118:2014. Artigo 17.3.5.2 } \\
\text { - Armadura inferior direção X: } \\
\text { - Armadura inferior direção Y: }\end{array}$ & $\begin{array}{l}\text { Calculado: } 0.0011 \\
\text { Mínimo: } 0.0007 \\
\text { Mínimo: } 0.0006\end{array}$ & $\begin{array}{l}\text { Passa } \\
\text { Passa }\end{array}$ \\
\hline $\begin{array}{l}\text { Diâmetro mínimo das barras: } \\
\text { - Malha inferior: } \\
\text { Critério da CYPE Ingenieros }\end{array}$ & $\begin{array}{l}\text { Mínimo: } 10 \mathrm{~mm} \\
\text { Calculado: } 10 \mathrm{~mm}\end{array}$ & Passa \\
\hline $\begin{array}{l}\text { Espaçamento máximo entre barras: } \\
\text { Critério da CYPE Ingenieros } \\
\text { - Armadura inferior direção X: } \\
\text { - Armadura inferior direção Y: }\end{array}$ & $\begin{array}{l}\text { Máximo: } 30 \mathrm{~cm} \\
\text { Calculado: } 28 \mathrm{~cm} \\
\text { Calculado: } 28 \mathrm{~cm}\end{array}$ & $\begin{array}{l}\text { Passa } \\
\text { Passa }\end{array}$ \\
\hline
\end{tabular}




\begin{tabular}{|c|c|c|}
\hline \multicolumn{3}{|l|}{$\begin{array}{l}\text { Referência: } P 7 \\
\text { Dimensões: } 90 \text { × } 90 \text { × } 30 / 20 \\
\text { Soldados: } X i: \varnothing 10 c / 28 \text { Yi: } \varnothing 10 c / 28\end{array}$} \\
\hline Verificação & Valores & Estado \\
\hline $\begin{array}{l}\text { Espaçamento mínimo entre barras: } \\
\text { Critério da CYPE Ingenieros, baseado em: J. Calavera. "Cálculo de } \\
\text { Estructuras de Cimentación". Capítulo } 3.16 \\
\text { - Armadura inferior direção X: } \\
\text { - Armadura inferior direção Y: }\end{array}$ & $\begin{array}{l}\text { Mínimo: } 10 \mathrm{~cm} \\
\text { Calculado: } 28 \mathrm{~cm} \\
\text { Calculado: } 28 \mathrm{~cm}\end{array}$ & $\begin{array}{l}\text { Passa } \\
\text { Passa }\end{array}$ \\
\hline $\begin{array}{l}\text { Comprimento de ancoragem: } \\
\text { Critério do livro "Cálculo de estructuras de cimentación", J. Calavera. } \\
\text { Ed. INTEMAC, } 1991 \\
\text { - Armadura inf. direção X para dir: } \\
\text { - Armadura inf. direção X para esq: } \\
\text { - Armadura inf. direção Y para cima: } \\
\text { - Armadura inf. direção Y para baixo: }\end{array}$ & $\begin{array}{l}\text { Calculado: } 32 \mathrm{~cm} \\
\text { Mínimo: } 11 \mathrm{~cm} \\
\text { Mínimo: } 22 \mathrm{~cm} \\
\text { Mínimo: } 11 \mathrm{~cm} \\
\text { Mínimo: } 22 \mathrm{~cm}\end{array}$ & $\begin{array}{l}\text { Passa } \\
\text { Passa } \\
\text { Passa } \\
\text { Passa }\end{array}$ \\
\hline $\begin{array}{l}\text { Comprimento mínimo das dobras: } \\
\text { - Armadura inf. direção X para dir: } \\
\text { - Armadura inf. direção X para esq: } \\
\text { - Armadura inf. direção Y para cima: } \\
\text { - Armadura inf. direção Y para baixo: }\end{array}$ & $\begin{array}{l}\text { Mínimo: } 11 \mathrm{~cm} \\
\text { Calculado: } 21 \mathrm{~cm} \\
\text { Calculado: } 21 \mathrm{~cm} \\
\text { Calculado: } 21 \mathrm{~cm} \\
\text { Calculado: } 21 \mathrm{~cm}\end{array}$ & $\begin{array}{l}\text { Passa } \\
\text { Passa } \\
\text { Passa } \\
\text { Passa }\end{array}$ \\
\hline $\begin{array}{l}\text { Todas as verificações foram } \\
\text { Referência: } \mathrm{P} 8 \\
\text { Dimensões: } 90 \times 90 \times 30 / 20 \\
\text { Soldados: } \mathrm{Xi}: \varnothing 10 \mathrm{c} / 28 \text { Yi: } \varnothing 10 \mathrm{c} / 28\end{array}$ & cumpridas & \\
\hline $\begin{array}{l}\text { Verificação } \\
\text { Ângulo máximo talude: } \\
\text { Critério da CYPE Ingenieros }\end{array}$ & $\begin{array}{l}\text { Máximo: } 30 \text { graus } \\
\text { Calculado: } 18.4349 \text { graus }\end{array}$ & Estado \\
\hline $\begin{array}{l}\text { Tensões sobre o terreno: } \\
\text { Critério da CYPE Ingenieros } \\
\text { - Tensão média em combinações fundamentais: } \\
\text { - Tensão máxima em combinações fundamentais: }\end{array}$ & $\begin{array}{l}\text { Máximo: } 0.24525 \mathrm{MPa} \\
\text { Calculado: } 0.082404 \mathrm{MPa} \\
\text { Máximo: } 0.306563 \mathrm{MPa} \\
\text { Calculado: } 0.167162 \mathrm{MPa}\end{array}$ & Passa \\
\hline $\begin{array}{l}\text { Tombamento da sapata: } \\
\text { Se o \% de reserva de segurança é maior que zero, pode ser dito que } \\
\text { os coeficientes de segurança ao tombamento são maiores que os } \\
\text { valores exatos exigidos para todas as combinações de equilíbrio. } \\
\text { - Na direção X: } \\
\text { - Na direção Y: }\end{array}$ & $\begin{array}{l}\text { Reserva segurança: } 191.2 \% \\
\text { Reserva segurança: } 1406.9 \%\end{array}$ & $\begin{array}{l}\text { Passa } \\
\text { Passa }\end{array}$ \\
\hline $\begin{array}{l}\text { Flexão na sapata: } \\
\text { - Na direção X: } \\
\text { - Na direção Y: }\end{array}$ & $\begin{array}{l}\text { Momento: } 11.53 \mathrm{kN} \cdot \mathrm{m} \\
\text { Momento: } 7.90 \mathrm{kN} \cdot \mathrm{m}\end{array}$ & $\begin{array}{l}\text { Passa } \\
\text { Passa }\end{array}$ \\
\hline & & \\
\hline
\end{tabular}




\begin{tabular}{|c|c|c|}
\hline $\begin{array}{l}\text { Referência: } \mathrm{P} 8 \\
\text { Dimensões: } 90 \text { × } 90 \text { × } 30 / 20 \\
\text { Soldados: Xi: Ø10c/28 Yi:Ø10c/28 }\end{array}$ & & \\
\hline Verificação & Valores & Estado \\
\hline $\begin{array}{l}\text { - Na direção } X: \\
\text { - Na direção Y: }\end{array}$ & $\begin{array}{l}\text { Cortante: } 17.56 \mathrm{kN} \\
\text { Cortante: } 11.28 \mathrm{kN}\end{array}$ & $\begin{array}{l}\text { Passa } \\
\text { Passa }\end{array}$ \\
\hline $\begin{array}{l}\text { Compressão oblíqua na sapata: } \\
\text { - Combinações fundamentais: } \\
\text { Critério da CYPE Ingenieros }\end{array}$ & $\begin{array}{l}\text { Máximo: } 5844.1 \mathrm{kN} / \mathrm{m}^{2} \\
\text { Calculado: } 497.8 \mathrm{kN} / \mathrm{m}^{2}\end{array}$ & Passa \\
\hline $\begin{array}{l}\text { Altura mínima: } \\
\text { Critério da CYPE Ingenieros }\end{array}$ & $\begin{array}{l}\text { Mínimo: } 15 \mathrm{~cm} \\
\text { Calculado: } 20 \mathrm{~cm}\end{array}$ & Passa \\
\hline $\begin{array}{l}\text { Espaço para ancorar arranques na fundação: } \\
\text { - P8: }\end{array}$ & $\begin{array}{l}\text { Mínimo: } 12 \mathrm{~cm} \\
\text { Calculado: } 24 \mathrm{~cm}\end{array}$ & Passa \\
\hline $\begin{array}{l}\text { Quantidade geométrica mínima: } \\
\text { Critério da CYPE Ingenieros } \\
\text { - Armadura inferior direção X: } \\
\text { - Armadura inferior direção Y: }\end{array}$ & $\begin{array}{l}\text { Mínimo: } 0.001 \\
\text { Calculado: } 0.001 \\
\text { Calculado: } 0.001\end{array}$ & $\begin{array}{l}\text { Passa } \\
\text { Passa }\end{array}$ \\
\hline $\begin{array}{l}\text { Quantia mínima necessária por flexão: } \\
\text { Norma Brasileira ABNT NBR 6118:2014. Artigo 17.3.5.2 } \\
\text { - Armadura inferior direção X: } \\
\text { - Armadura inferior direção Y: }\end{array}$ & $\begin{array}{l}\text { Calculado: } 0.0011 \\
\text { Mínimo: } 0.0006 \\
\text { Mínimo: } 0.0004\end{array}$ & $\begin{array}{l}\text { Passa } \\
\text { Passa }\end{array}$ \\
\hline $\begin{array}{l}\text { Diâmetro mínimo das barras: } \\
\text { - Malha inferior: } \\
\text { Critério da CYPE Ingenieros }\end{array}$ & $\begin{array}{l}\text { Mínimo: } 10 \mathrm{~mm} \\
\text { Calculado: } 10 \mathrm{~mm}\end{array}$ & Passa \\
\hline $\begin{array}{l}\text { Espaçamento máximo entre barras: } \\
\text { Critério da CYPE Ingenieros } \\
\text { - Armadura inferior direção X: } \\
\text { - Armadura inferior direção Y: }\end{array}$ & $\begin{array}{l}\text { Máximo: } 30 \mathrm{~cm} \\
\text { Calculado: } 28 \mathrm{~cm} \\
\text { Calculado: } 28 \mathrm{~cm}\end{array}$ & $\begin{array}{l}\text { Passa } \\
\text { Passa }\end{array}$ \\
\hline $\begin{array}{l}\text { Espaçamento mínimo entre barras: } \\
\text { Critério da CYPE Ingenieros, baseado em: J. Calavera. "Cálculo de } \\
\text { Estructuras de Cimentación". Capítulo } 3.16 \\
\text { - Armadura inferior direção X: } \\
\text { - Armadura inferior direção Y: }\end{array}$ & $\begin{array}{l}\text { Mínimo: } 10 \mathrm{~cm} \\
\text { Calculado: } 28 \mathrm{~cm} \\
\text { Calculado: } 28 \mathrm{~cm}\end{array}$ & $\begin{array}{l}\text { Passa } \\
\text { Passa }\end{array}$ \\
\hline $\begin{array}{l}\text { Comprimento de ancoragem: } \\
\text { Critério do livro "Cálculo de estructuras de cimentación", J. Calavera. } \\
\text { Ed. INTEMAC, } 1991 \\
\text { - Armadura inf. direção X para dir: } \\
\text { - Armadura inf. direção X para esq: } \\
\text { - Armadura inf. direção Y para cima: } \\
\text { - Armadura inf. direção Y para baixo: }\end{array}$ & $\begin{array}{l}\text { Calculado: } 32 \mathrm{~cm} \\
\text { Mínimo: } 22 \mathrm{~cm} \\
\text { Mínimo: } 11 \mathrm{~cm} \\
\text { Mínimo: } 22 \mathrm{~cm} \\
\text { Mínimo: } 11 \mathrm{~cm}\end{array}$ & $\begin{array}{l}\text { Passa } \\
\text { Passa } \\
\text { Passa } \\
\text { Passa }\end{array}$ \\
\hline Comprimento mínimo das dobras: & Mínimo: $11 \mathrm{~cm}$ & \\
\hline
\end{tabular}




\begin{tabular}{|c|c|c|}
\hline \multicolumn{3}{|l|}{$\begin{array}{l}\text { Referência: P8 } \\
\text { Dimensões: } 90 \text { x } 90 \text { × } 30 / 20 \\
\text { Soldados: Xi: } \varnothing 10 c / 28 \text { Yi: Ø10c/28 }\end{array}$} \\
\hline Verificação & Valores & Estado \\
\hline - Armadura inf. direção X para dir: & Calculado: $21 \mathrm{~cm}$ & Passa \\
\hline -Armadura inf. direção $X$ para esq: & Calculado: $21 \mathrm{~cm}$ & Passa \\
\hline - Armadura inf. direção Y para cima: & Calculado: $21 \mathrm{~cm}$ & Passa \\
\hline - Armadura inf. direção Y para baixo: & Calculado: $21 \mathrm{~cm}$ & Passa \\
\hline \multicolumn{3}{|c|}{ Todas as verificações foram cumpridas } \\
\hline \multicolumn{3}{|l|}{$\begin{array}{l}\text { Referência: P9 } \\
\text { Dimensões: } 90 \text { x } 90 \text { x } 30 / 20 \\
\text { Soldados: Xi: Ø10c/28 Yi:Ø10c/28 }\end{array}$} \\
\hline Verificação & Valores & Estado \\
\hline $\begin{array}{l}\text { Ângulo máximo talude: } \\
\text { Critério da CYPE Ingenieros }\end{array}$ & $\begin{array}{l}\text { Máximo: } 30 \text { graus } \\
\text { Calculado: } 18.4349 \text { graus }\end{array}$ & Passa \\
\hline $\begin{array}{l}\text { Tensões sobre o terreno: } \\
\text { Critério da CYPE Ingenieros } \\
\text { - Tensão média em combinações fundamentais: } \\
\text {-Tensão máxima em combinações fundamentais: }\end{array}$ & $\begin{array}{l}\text { Máximo: } 0.24525 \mathrm{MPa} \\
\text { Calculado: } 0.0410058 \mathrm{MPa} \\
\text { Máximo: } 0.306563 \mathrm{MPa} \\
\text { Calculado: } 0.0959418 \mathrm{MPa}\end{array}$ & $\begin{array}{l}\text { Passa } \\
\text { Passa }\end{array}$ \\
\hline $\begin{array}{l}\text { Tombamento da sapata: } \\
\text { Se o \% de reserva de segurança é maior que zero, pode ser dito que } \\
\text { os coeficientes de segurança ao tombamento são maiores que os } \\
\text { valores exatos exigidos para todas as combinações de equilibrio. } \\
\text { - Na direção X: } \\
\text { - Na direção Y: }\end{array}$ & $\begin{array}{l}\text { Reserva segurança: } 695.6 \% \\
\text { Reserva segurança: } 104.3 \%\end{array}$ & $\begin{array}{l}\text { Passa } \\
\text { Passa }\end{array}$ \\
\hline $\begin{array}{l}\text { Flexão na sapata: } \\
\text { - Na direção } X: \\
\text { - Na direção Y: }\end{array}$ & $\begin{array}{l}\text { Momento: } 3.00 \mathrm{kN} \cdot \mathrm{m} \\
\text { Momento: } 5.45 \mathrm{kN} \cdot \mathrm{m}\end{array}$ & $\begin{array}{l}\text { Passa } \\
\text { Passa }\end{array}$ \\
\hline $\begin{array}{l}\text { Cortante na sapata: } \\
\text { - Na direção X: } \\
\text { - Na direção Y: }\end{array}$ & $\begin{array}{l}\text { Cortante: } 4.51 \mathrm{kN} \\
\text { Cortante: } 8.73 \mathrm{kN}\end{array}$ & $\begin{array}{l}\text { Passa } \\
\text { Passa }\end{array}$ \\
\hline $\begin{array}{l}\text { Compressão oblíqua na sapata: } \\
\text { - Combinações fundamentais: } \\
\text { Critério da CYPE Ingenieros }\end{array}$ & $\begin{array}{l}\text { Máximo: } 5844.1 \mathrm{kN} / \mathrm{m}^{2} \\
\text { Calculado: } 161.3 \mathrm{kN} / \mathrm{m}^{2}\end{array}$ & Passa \\
\hline $\begin{array}{l}\text { Altura mínima: } \\
\text { Critério da CYPE Ingenieros }\end{array}$ & $\begin{array}{l}\text { Mínimo: } 15 \mathrm{~cm} \\
\text { Calculado: } 20 \mathrm{~cm}\end{array}$ & Passa \\
\hline $\begin{array}{l}\text { Espaço para ancorar arranques na fundação: } \\
\text { - P9: }\end{array}$ & $\begin{array}{l}\text { Mínimo: } 12 \mathrm{~cm} \\
\text { Calculado: } 24 \mathrm{~cm}\end{array}$ & Passa \\
\hline $\begin{array}{l}\text { Quantidade geométrica mínima: } \\
\text { Critério da CYPE Ingenieros } \\
\text { - Armadura inferior direção X: }\end{array}$ & $\begin{array}{l}\text { Mínimo: } 0.001 \\
\text { Calculado: } 0.001\end{array}$ & Passa \\
\hline
\end{tabular}




\begin{tabular}{|c|c|c|}
\hline $\begin{array}{l}\text { Referência: P9 } \\
\text { Dimensões: } 90 \text { × } 90 \text { × } 30 / 20 \\
\text { Soldados: Xi: Ø10c/28 Yi:Ø10c/28 }\end{array}$ & & \\
\hline Verificação & Valores & Estado \\
\hline - Armadura inferior direção Y: & Calculado: 0.001 & Passa \\
\hline $\begin{array}{l}\text { Quantia mínima necessária por flexão: } \\
\text { Norma Brasileira ABNT NBR 6118:2014. Artigo 17.3.5.2 } \\
\text { - Armadura inferior direção X: } \\
\text { - Armadura inferior direção Y: }\end{array}$ & $\begin{array}{l}\text { Calculado: } 0.0011 \\
\text { Mínimo: } 0.0002 \\
\text { Mínimo: } 0.0003\end{array}$ & $\begin{array}{l}\text { Passa } \\
\text { Passa }\end{array}$ \\
\hline $\begin{array}{l}\text { Diâmetro mínimo das barras: } \\
\text { - Malha inferior: } \\
\text { Critério da CYPE Ingenieros }\end{array}$ & $\begin{array}{l}\text { Mínimo: } 10 \mathrm{~mm} \\
\text { Calculado: } 10 \mathrm{~mm}\end{array}$ & Passa \\
\hline $\begin{array}{l}\text { Espaçamento máximo entre barras: } \\
\text { Critério da CYPE Ingenieros } \\
\text { - Armadura inferior direção } \mathrm{X} \text { : } \\
\text { - Armadura inferior direção Y: }\end{array}$ & $\begin{array}{l}\text { Máximo: } 30 \mathrm{~cm} \\
\text { Calculado: } 28 \mathrm{~cm} \\
\text { Calculado: } 28 \mathrm{~cm}\end{array}$ & $\begin{array}{l}\text { Passa } \\
\text { Passa }\end{array}$ \\
\hline $\begin{array}{l}\text { Espaçamento mínimo entre barras: } \\
\text { Critério da CYPE Ingenieros, baseado em: J. Calavera. "Cálculo de } \\
\text { Estructuras de Cimentación". Capítulo 3.16 } \\
\text { - Armadura inferior direção X: } \\
\text { - Armadura inferior direção Y: }\end{array}$ & $\begin{array}{l}\text { Mínimo: } 10 \mathrm{~cm} \\
\text { Calculado: } 28 \mathrm{~cm} \\
\text { Calculado: } 28 \mathrm{~cm}\end{array}$ & $\begin{array}{l}\text { Passa } \\
\text { Passa }\end{array}$ \\
\hline $\begin{array}{l}\text { Comprimento de ancoragem: } \\
\text { Critério do livro "Cálculo de estructuras de cimentación", J. Calavera. } \\
\text { Ed. INTEMAC, } 1991 \\
\text { - Armadura inf. direção X para dir: } \\
\text { - Armadura inf. direção X para esq: } \\
\text { - Armadura inf. direção Y para cima: } \\
\text { - Armadura inf. direção Y para baixo: }\end{array}$ & $\begin{array}{l}\text { Mínimo: } 11 \mathrm{~cm} \\
\text { Calculado: } 11 \mathrm{~cm} \\
\text { Calculado: } 11 \mathrm{~cm} \\
\text { Calculado: } 11 \mathrm{~cm} \\
\text { Calculado: } 11 \mathrm{~cm}\end{array}$ & $\begin{array}{l}\text { Passa } \\
\text { Passa } \\
\text { Passa } \\
\text { Passa }\end{array}$ \\
\hline $\begin{array}{l}\text { Todas as verificações foran } \\
\text { Referência: P10 } \\
\text { Dimensões: } 90 \times 90 \times 30 / 20 \\
\text { Soldados: } \mathrm{Xi}: \varnothing 10 \mathrm{c} / 28 \mathrm{Yi}: \varnothing 10 \mathrm{c} / 28\end{array}$ & cumpridas & \\
\hline Verificação & Valores & Estado \\
\hline $\begin{array}{l}\text { Ângulo máximo talude: } \\
\text { Critério da CYPE Ingenieros }\end{array}$ & $\begin{array}{l}\text { Máximo: } 30 \text { graus } \\
\text { Calculado: } 18.4349 \text { graus }\end{array}$ & Passa \\
\hline $\begin{array}{l}\text { Tensões sobre o terreno: } \\
\text { Critério da CYPE Ingenieros } \\
\text { - Tensão média em combinações fundamentais: } \\
\text { - Tensão máxima em combinações fundamentais: }\end{array}$ & $\begin{array}{l}\text { Máximo: } 0.24525 \mathrm{MPa} \\
\text { Calculado: } 0.101435 \mathrm{MPa} \\
\text { Máximo: } 0.306563 \mathrm{MPa} \\
\text { Calculado: } 0.207972 \mathrm{MPa}\end{array}$ & Passa \\
\hline
\end{tabular}




\begin{tabular}{|c|c|c|}
\hline \multicolumn{3}{|l|}{$\begin{array}{l}\text { Referência: } \mathrm{P} 10 \\
\text { Dimensões: } 90 \times 90 \times 30 / 20 \\
\text { Soldados: Xi: } \varnothing 10 \mathrm{c} / 28 \text { Yi: } \varnothing 10 \mathrm{c} / 28\end{array}$} \\
\hline Verificação & Valores & Estado \\
\hline $\begin{array}{l}\text { Tombamento da sapata: } \\
\text { Se o \% de reserva de segurança é maior que zero, pode ser dito que } \\
\text { os coeficientes de segurança ao tombamento são maiores que os } \\
\text { valores exatos exigidos para todas as combinaçôes de equilíbrio. } \\
\text { - Na direção X: } \\
\text { - Na direção Y: }\end{array}$ & $\begin{array}{l}\text { Reserva segurança: } 304.2 \% \\
\text { Reserva segurança: } 583.4 \%\end{array}$ & Passa \\
\hline $\begin{array}{l}\text { Flexão na sapata: } \\
\text { - Na direção X: } \\
\text { - Na direção Y: }\end{array}$ & $\begin{array}{l}\text { Momento: } 13.05 \mathrm{kN} \cdot \mathrm{m} \\
\text { Momento: } 11.26 \mathrm{kN} \cdot \mathrm{m}\end{array}$ & $\begin{array}{l}\text { Passa } \\
\text { Passa }\end{array}$ \\
\hline $\begin{array}{l}\text { Cortante na sapata: } \\
\text { - Na direção X: } \\
\text { - Na direção Y: }\end{array}$ & $\begin{array}{l}\text { Cortante: } 19.52 \mathrm{kN} \\
\text { Cortante: } 16.48 \mathrm{kN}\end{array}$ & $\begin{array}{l}\text { Passa } \\
\text { Passa }\end{array}$ \\
\hline $\begin{array}{l}\text { Compressão oblíqua na sapata: } \\
\text { - Combinações fundamentais: } \\
\text { Critério da CYPE Ingenieros }\end{array}$ & $\begin{array}{l}\text { Máximo: } 5844.1 \mathrm{kN} / \mathrm{m}^{2} \\
\text { Calculado: } 621.3 \mathrm{kN} / \mathrm{m}^{2}\end{array}$ & Passa \\
\hline $\begin{array}{l}\text { Altura mínima: } \\
\text { Critério da CYPE Ingenieros }\end{array}$ & $\begin{array}{l}\text { Mínimo: } 15 \mathrm{~cm} \\
\text { Calculado: } 20 \mathrm{~cm}\end{array}$ & Passa \\
\hline $\begin{array}{l}\text { Espaço para ancorar arranques na fundação: } \\
\text { - P10: }\end{array}$ & $\begin{array}{l}\text { Mínimo: } 12 \mathrm{~cm} \\
\text { Calculado: } 24 \mathrm{~cm}\end{array}$ & Passa \\
\hline $\begin{array}{l}\text { Quantidade geométrica mínima: } \\
\text { Critério da CYPE Ingenieros } \\
\text { - Armadura inferior direção X: } \\
\text { - Armadura inferior direção Y: }\end{array}$ & $\begin{array}{l}\text { Mínimo: } 0.001 \\
\text { Calculado: } 0.001 \\
\text { Calculado: } 0.001\end{array}$ & $\begin{array}{l}\text { Passa } \\
\text { Passa }\end{array}$ \\
\hline $\begin{array}{l}\text { Quantia mínima necessária por flexão: } \\
\text { Norma Brasileira ABNT NBR 6118:2014. Artigo 17.3.5.2 } \\
\text { - Armadura inferior direção X: } \\
\text { - Armadura inferior direção Y: }\end{array}$ & $\begin{array}{l}\text { Calculado: } 0.0011 \\
\text { Mínimo: } 0.0006 \\
\text { Mínimo: } 0.0005\end{array}$ & $\begin{array}{l}\text { Passa } \\
\text { Passa }\end{array}$ \\
\hline $\begin{array}{l}\text { Diâmetro mínimo das barras: } \\
\text { - Malha inferior: } \\
\text { Critério da CYPE Ingenieros }\end{array}$ & $\begin{array}{l}\text { Mínimo: } 10 \mathrm{~mm} \\
\text { Calculado: } 10 \mathrm{~mm}\end{array}$ & Passa \\
\hline $\begin{array}{l}\text { Espaçamento máximo entre barras: } \\
\text { Critério da CYPE Ingenieros } \\
\text {-Armadura inferior direção X: } \\
\text {-Armadura inferior direção Y: }\end{array}$ & $\begin{array}{l}\text { Máximo: } 30 \mathrm{~cm} \\
\text { Calculado: } 28 \mathrm{~cm} \\
\text { Calculado: } 28 \mathrm{~cm}\end{array}$ & $\begin{array}{l}\text { Passa } \\
\text { Passa }\end{array}$ \\
\hline $\begin{array}{l}\text { Espaçamento mínimo entre barras: } \\
\text { Critério da CYPE Ingenieros, baseado em: J. Calavera. "Cálculo de } \\
\text { Estructuras de Cimentación". Capítulo 3.16 } \\
\text { - Armadura inferior direção X: }\end{array}$ & $\begin{array}{l}\text { Mínimo: } 10 \mathrm{~cm} \\
\text { Calculado: } 28 \mathrm{~cm}\end{array}$ & Passa \\
\hline
\end{tabular}




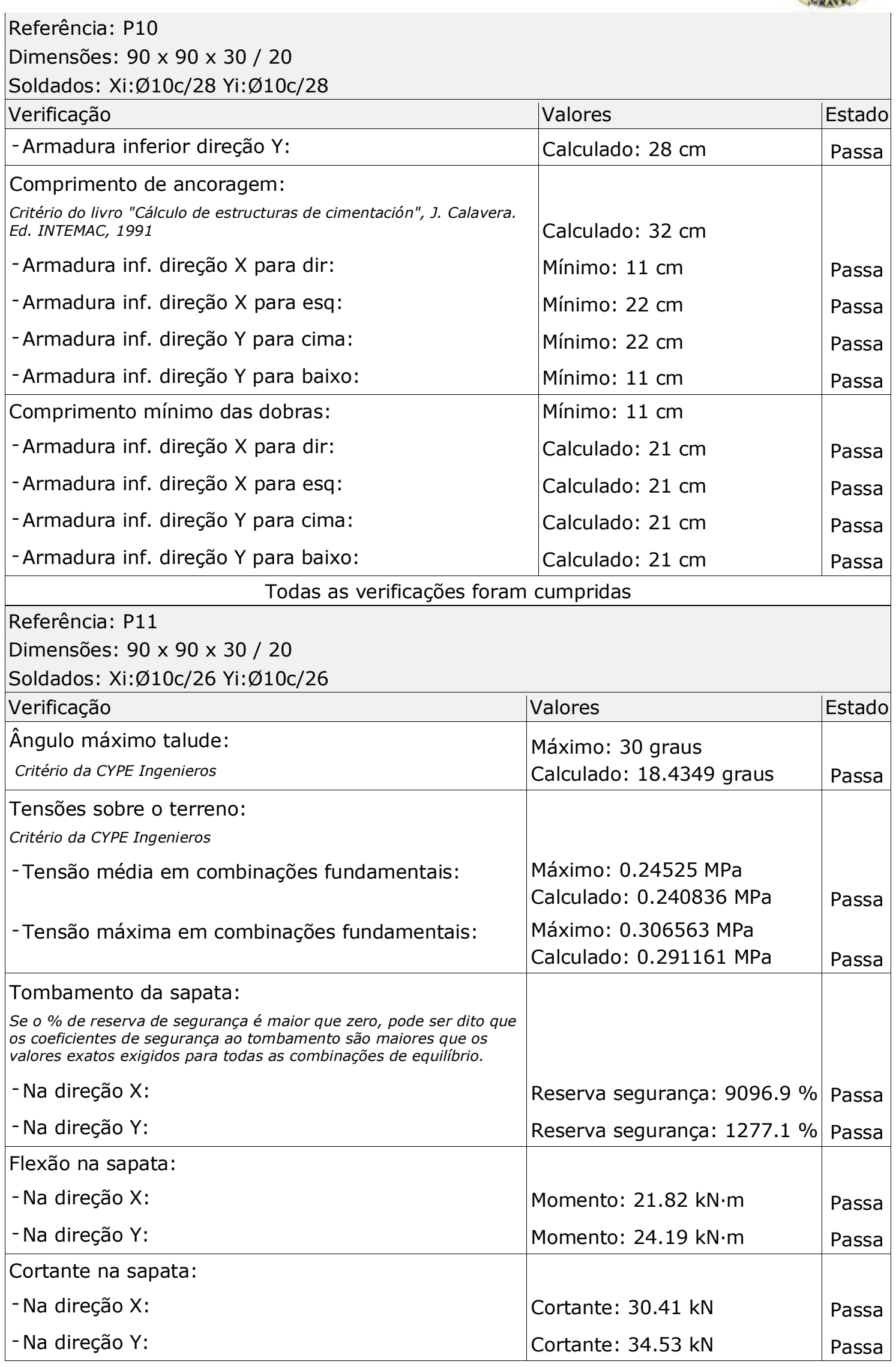




\begin{tabular}{|c|c|c|}
\hline $\begin{array}{l}\text { Referência: P11 } \\
\text { Dimensões: } 90 \text { x } 90 \text { x } 30 / 20 \\
\text { Soldados: Xi: Ø10c/26 Yi:Ø10c/26 }\end{array}$ & & \\
\hline Verificação & Valores & Estado \\
\hline $\begin{array}{l}\text { Compressão oblíqua na sapata: } \\
\text { - Combinações fundamentais: } \\
\text { Critério da CYPE Ingenieros }\end{array}$ & $\begin{array}{l}\text { Máximo: } 5844.1 \mathrm{kN} / \mathrm{m}^{2} \\
\text { Calculado: } 1531.1 \mathrm{kN} / \mathrm{m}^{2}\end{array}$ & Passa \\
\hline $\begin{array}{l}\text { Altura mínima: } \\
\text { Critério da CYPE Ingenieros }\end{array}$ & $\begin{array}{l}\text { Mínimo: } 15 \mathrm{~cm} \\
\text { Calculado: } 20 \mathrm{~cm}\end{array}$ & Passa \\
\hline $\begin{array}{l}\text { Espaço para ancorar arranques na fundação: } \\
\text { - P11: }\end{array}$ & $\begin{array}{l}\text { Mínimo: } 12 \mathrm{~cm} \\
\text { Calculado: } 24 \mathrm{~cm}\end{array}$ & Passa \\
\hline $\begin{array}{l}\text { Quantidade geométrica mínima: } \\
\text { Critério da CYPE Ingenieros } \\
\text { - Armadura inferior direção X: } \\
\text { - Armadura inferior direção Y: }\end{array}$ & $\begin{array}{l}\text { Mínimo: } 0.001 \\
\text { Calculado: } 0.0011 \\
\text { Calculado: } 0.0011\end{array}$ & $\begin{array}{l}\text { Passa } \\
\text { Passa }\end{array}$ \\
\hline $\begin{array}{l}\text { Quantia mínima necessária por flexão: } \\
\text { Norma Brasileira ABNT NBR 6118:2014. Artigo 17.3.5.2 } \\
\text { - Armadura inferior direção X: } \\
\text { - Armadura inferior direção Y: }\end{array}$ & $\begin{array}{l}\text { Calculado: } 0.0012 \\
\text { Mínimo: } 0.001 \\
\text { Mínimo: } 0.0011\end{array}$ & $\begin{array}{l}\text { Passa } \\
\text { Passa }\end{array}$ \\
\hline $\begin{array}{l}\text { Diâmetro mínimo das barras: } \\
\text { - Malha inferior: } \\
\text { Critério da CYPE Ingenieros }\end{array}$ & $\begin{array}{l}\text { Mínimo: } 10 \mathrm{~mm} \\
\text { Calculado: } 10 \mathrm{~mm}\end{array}$ & Passa \\
\hline $\begin{array}{l}\text { Espaçamento máximo entre barras: } \\
\text { Critério da CYPE Ingenieros } \\
\text { - Armadura inferior direção X: } \\
\text { - Armadura inferior direção Y: }\end{array}$ & $\begin{array}{l}\text { Máximo: } 30 \mathrm{~cm} \\
\text { Calculado: } 26 \mathrm{~cm} \\
\text { Calculado: } 26 \mathrm{~cm}\end{array}$ & $\begin{array}{l}\text { Passa } \\
\text { Passa }\end{array}$ \\
\hline $\begin{array}{l}\text { Espaçamento mínimo entre barras: } \\
\text { Critério da CYPE Ingenieros, baseado em: J. Calavera. "Cálculo de } \\
\text { Estructuras de Cimentación". Capítulo 3.16 } \\
\text { - Armadura inferior direção X: } \\
\text { - Armadura inferior direção Y: }\end{array}$ & $\begin{array}{l}\text { Mínimo: } 10 \mathrm{~cm} \\
\text { Calculado: } 26 \mathrm{~cm} \\
\text { Calculado: } 26 \mathrm{~cm}\end{array}$ & $\begin{array}{l}\text { Passa } \\
\text { Passa }\end{array}$ \\
\hline $\begin{array}{l}\text { Comprimento de ancoragem: } \\
\text { Critério do livro "Cálculo de estructuras de cimentación", J. Calavera. } \\
\text { Ed. INTEMAC, } 1991 \\
\text { - Armadura inf. direção X para dir: } \\
\text { - Armadura inf. direção X para esq: } \\
\text { - Armadura inf. direção Y para cima: } \\
\text { - Armadura inf. direção Y para baixo: }\end{array}$ & $\begin{array}{l}\text { Calculado: } 32 \mathrm{~cm} \\
\text { Mínimo: } 22 \mathrm{~cm} \\
\text { Mínimo: } 22 \mathrm{~cm} \\
\text { Mínimo: } 25 \mathrm{~cm} \\
\text { Mínimo: } 22 \mathrm{~cm}\end{array}$ & $\begin{array}{l}\text { Passa } \\
\text { Passa } \\
\text { Passa } \\
\text { Passa }\end{array}$ \\
\hline $\begin{array}{l}\text { Comprimento mínimo das dobras: } \\
\text { - Armadura inf. direção X para dir: } \\
\text { - Armadura inf. direção X para esq: }\end{array}$ & $\begin{array}{l}\text { Mínimo: } 11 \mathrm{~cm} \\
\text { Calculado: } 21 \mathrm{~cm} \\
\text { Calculado: } 21 \mathrm{~cm}\end{array}$ & $\begin{array}{l}\text { Passa } \\
\text { Passa }\end{array}$ \\
\hline
\end{tabular}




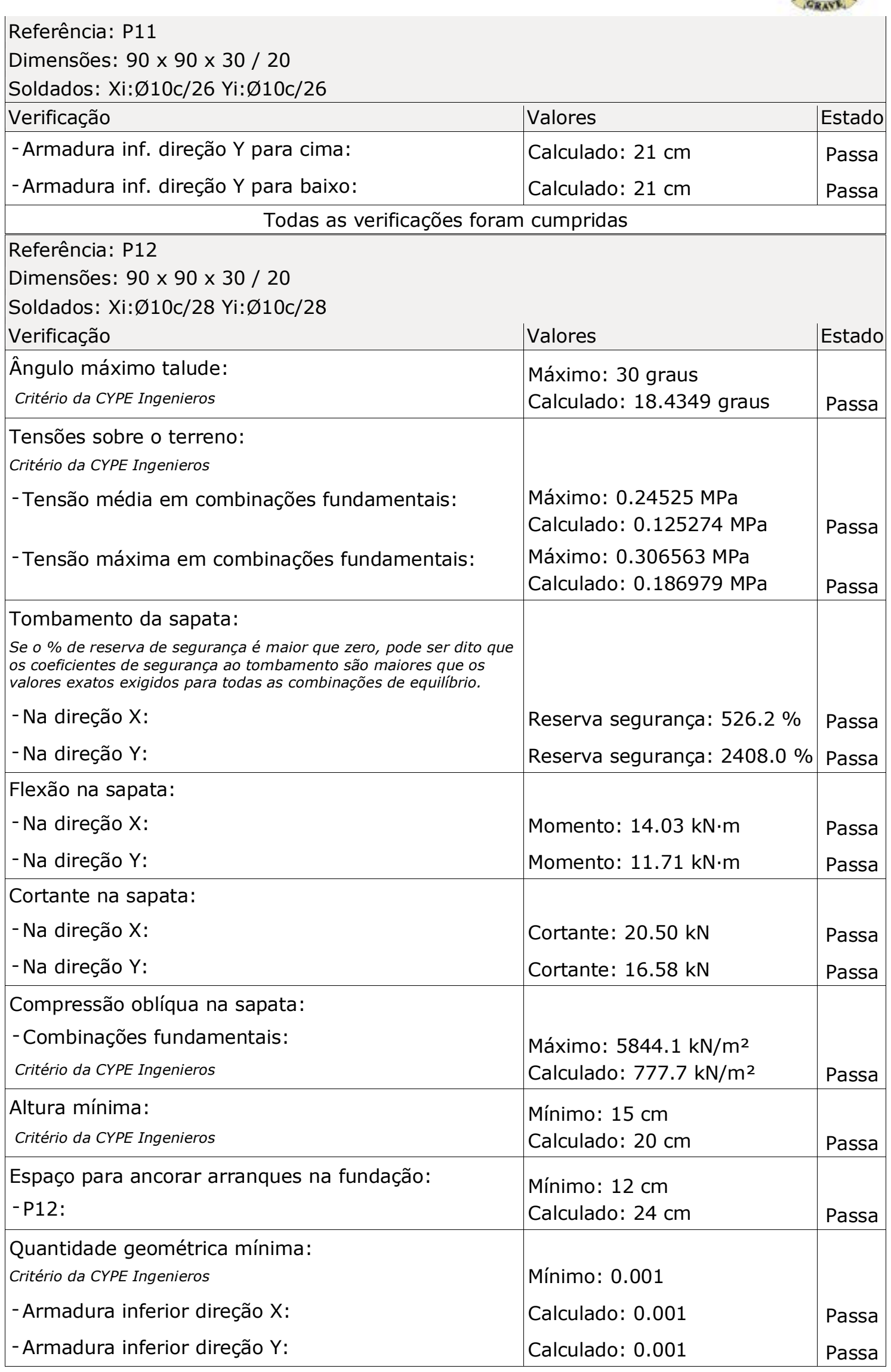




\begin{tabular}{|c|c|c|}
\hline $\begin{array}{l}\text { Referência: P12 } \\
\text { Dimensões: } 90 \times 90 \times 30 / 20 \\
\text { Soldados: Xi: } \varnothing 10 c / 28 \text { Yi: Ø10c/28 }\end{array}$ & & \\
\hline Verificação & Valores & Estado \\
\hline $\begin{array}{l}\text { Quantia mínima necessária por flexão: } \\
\text { Norma Brasileira ABNT NBR 6118:2014. Artigo 17.3.5.2 } \\
\text { - Armadura inferior direção X: } \\
\text { - Armadura inferior direção Y: }\end{array}$ & $\begin{array}{l}\text { Calculado: } 0.0011 \\
\text { Mínimo: } 0.0007 \\
\text { Mínimo: } 0.0006\end{array}$ & $\begin{array}{l}\text { Passa } \\
\text { Passa }\end{array}$ \\
\hline $\begin{array}{l}\text { Diâmetro mínimo das barras: } \\
\text { - Malha inferior: } \\
\text { Critério da CYPE Ingenieros }\end{array}$ & $\begin{array}{l}\text { Mínimo: } 10 \mathrm{~mm} \\
\text { Calculado: } 10 \mathrm{~mm}\end{array}$ & Passa \\
\hline $\begin{array}{l}\text { Espaçamento máximo entre barras: } \\
\text { Critério da CYPE Ingenieros } \\
\text { - Armadura inferior direção X: } \\
\text { - Armadura inferior direção Y: }\end{array}$ & $\begin{array}{l}\text { Máximo: } 30 \mathrm{~cm} \\
\text { Calculado: } 28 \mathrm{~cm} \\
\text { Calculado: } 28 \mathrm{~cm}\end{array}$ & $\begin{array}{l}\text { Passa } \\
\text { Passa }\end{array}$ \\
\hline $\begin{array}{l}\text { Espaçamento mínimo entre barras: } \\
\text { Critério da CYPE Ingenieros, baseado em: J. Calavera. "Cálculo de } \\
\text { Estructuras de Cimentación". Capítulo 3.16 } \\
\text { - Armadura inferior direção X: } \\
\text { - Armadura inferior direção Y: }\end{array}$ & $\begin{array}{l}\text { Mínimo: } 10 \mathrm{~cm} \\
\text { Calculado: } 28 \mathrm{~cm} \\
\text { Calculado: } 28 \mathrm{~cm}\end{array}$ & $\begin{array}{l}\text { Passa } \\
\text { Passa }\end{array}$ \\
\hline $\begin{array}{l}\text { Comprimento de ancoragem: } \\
\text { Critério do livro "Cálculo de estructuras de cimentación", J. Calavera. } \\
\text { Ed. INTEMAC, } 1991 \\
\text { - Armadura inf. direção X para dir: } \\
\text { - Armadura inf. direção X para esq: } \\
\text { - Armadura inf. direção Y para cima: } \\
\text { - Armadura inf. direção Y para baixo: }\end{array}$ & $\begin{array}{l}\text { Mínimo: } 22 \mathrm{~cm} \\
\text { Calculado: } 32 \mathrm{~cm} \\
\text { Calculado: } 32 \mathrm{~cm} \\
\text { Calculado: } 32 \mathrm{~cm} \\
\text { Calculado: } 32 \mathrm{~cm}\end{array}$ & $\begin{array}{l}\text { Passa } \\
\text { Passa } \\
\text { Passa } \\
\text { Passa }\end{array}$ \\
\hline $\begin{array}{l}\text { Comprimento mínimo das dobras: } \\
\text { - Armadura inf. direção X para dir: } \\
\text { - Armadura inf. direção X para esq: } \\
\text { - Armadura inf. direção Y para cima: } \\
\text { - Armadura inf. direção Y para baixo: }\end{array}$ & $\begin{array}{l}\text { Mínimo: } 11 \mathrm{~cm} \\
\text { Calculado: } 21 \mathrm{~cm} \\
\text { Calculado: } 21 \mathrm{~cm} \\
\text { Calculado: } 21 \mathrm{~cm} \\
\text { Calculado: } 21 \mathrm{~cm}\end{array}$ & $\begin{array}{l}\text { Passa } \\
\text { Passa } \\
\text { Passa } \\
\text { Passa }\end{array}$ \\
\hline Todas as verificações foram & cumpridas & \\
\hline $\begin{array}{l}\text { Referência: P13 } \\
\text { Dimensões: } 90 \text { × } 90 \text { × } 30 / 20 \\
\text { Soldados: Xi: Ø10c/28 Yi: Ø10c/28 } \\
\text { Verificação }\end{array}$ & Valores & Estado \\
\hline $\begin{array}{l}\text { Ângulo máximo talude: } \\
\text { Critério da CYPE Ingenieros }\end{array}$ & $\begin{array}{l}\text { Máximo: } 30 \text { graus } \\
\text { Calculado: } 18.4349 \text { graus }\end{array}$ & Passa \\
\hline $\begin{array}{l}\text { Tensões sobre o terreno: } \\
\text { Critério da CYPE Ingenieros } \\
\text { - Tensão média em combinações fundamentais: }\end{array}$ & $\begin{array}{l}\text { Máximo: } 0.24525 \mathrm{MPa} \\
\text { Calculado: } 0.0920178 \mathrm{MPa}\end{array}$ & Passa \\
\hline
\end{tabular}




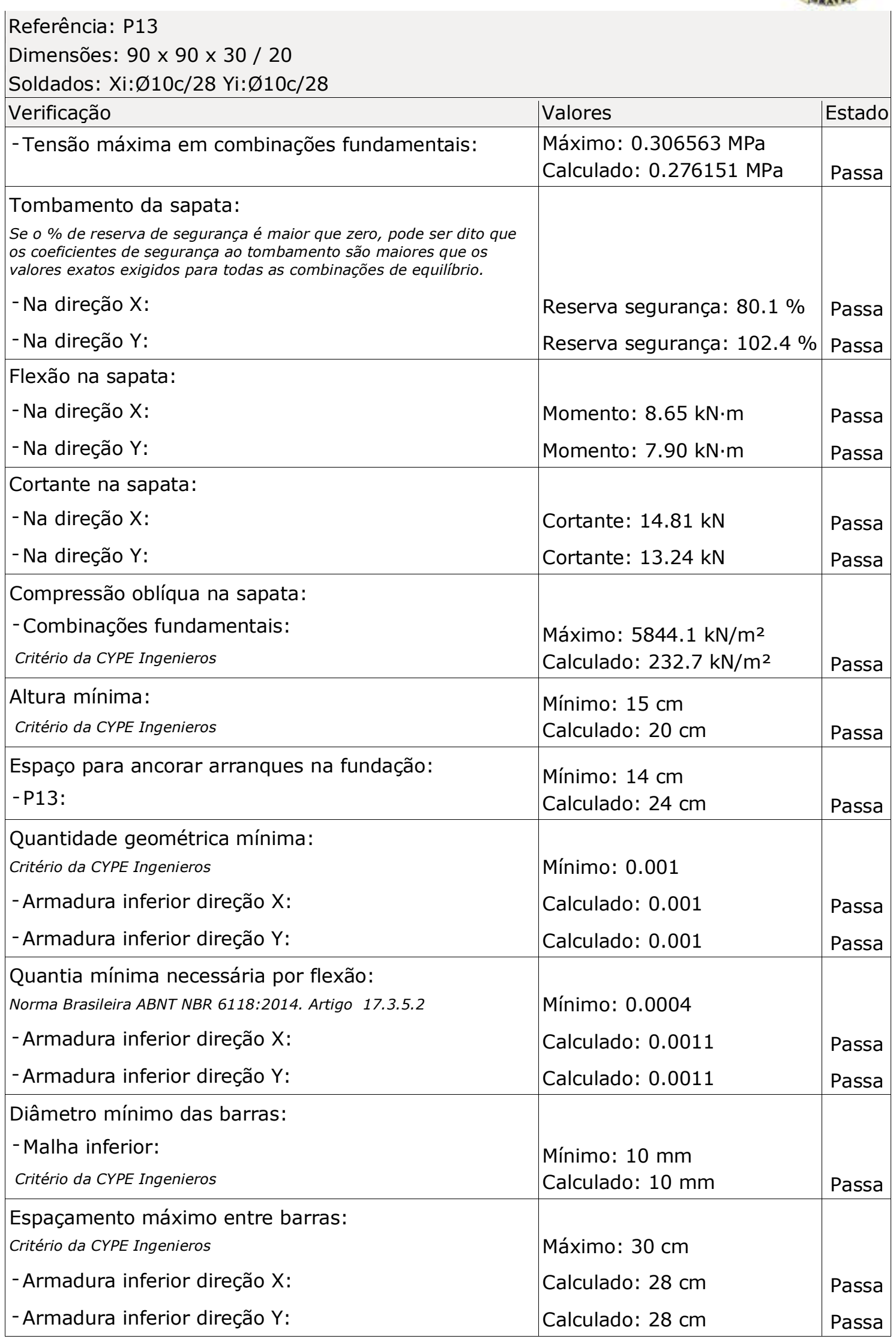




\begin{tabular}{|c|c|c|}
\hline \multicolumn{3}{|l|}{$\begin{array}{l}\text { Referência: P13 } \\
\text { Dimensões: } 90 \text { × } 90 \text { × } 30 / 20 \\
\text { Soldados: Xi: } \varnothing 10 c / 28 \text { Yi: Ø10c/28 }\end{array}$} \\
\hline Verificação & Valores & Estado \\
\hline $\begin{array}{l}\text { Espaçamento mínimo entre barras: } \\
\text { Critério da CYPE Ingenieros, baseado em: J. Calavera. "Cálculo de } \\
\text { Estructuras de Cimentación". Capítulo 3.16 } \\
\text { - Armadura inferior direção X: } \\
\text { - Armadura inferior direção Y: }\end{array}$ & $\begin{array}{l}\text { Mínimo: } 10 \mathrm{~cm} \\
\text { Calculado: } 28 \mathrm{~cm} \\
\text { Calculado: } 28 \mathrm{~cm}\end{array}$ & $\begin{array}{l}\text { Passa } \\
\text { Passa }\end{array}$ \\
\hline $\begin{array}{l}\text { Comprimento de ancoragem: } \\
\text { Critério do livro "Ć́lculo de estructuras de cimentación", J. Calavera. } \\
\text { Ed. INTEMAC, } 1991 \\
\text { - Armadura inf. direção X para dir: } \\
\text { - Armadura inf. direção X para esq: } \\
\text { - Armadura inf. direção Y para cima: } \\
\text { - Armadura inf. direção Y para baixo: }\end{array}$ & $\begin{array}{l}\text { Calculado: } 32 \mathrm{~cm} \\
\text { Mínimo: } 22 \mathrm{~cm} \\
\text { Mínimo: } 11 \mathrm{~cm} \\
\text { Mínimo: } 22 \mathrm{~cm} \\
\text { Mínimo: } 11 \mathrm{~cm}\end{array}$ & $\begin{array}{l}\text { Passa } \\
\text { Passa } \\
\text { Passa } \\
\text { Passa }\end{array}$ \\
\hline $\begin{array}{l}\text { Comprimento mínimo das dobras: } \\
\text { - Armadura inf. direção X para dir: } \\
\text { - Armadura inf. direção X para esq: } \\
\text { - Armadura inf. direção Y para cima: } \\
\text { - Armadura inf. direção Y para baixo: }\end{array}$ & $\begin{array}{l}\text { Mínimo: } 11 \mathrm{~cm} \\
\text { Calculado: } 21 \mathrm{~cm} \\
\text { Calculado: } 21 \mathrm{~cm} \\
\text { Calculado: } 21 \mathrm{~cm} \\
\text { Calculado: } 21 \mathrm{~cm}\end{array}$ & $\begin{array}{l}\text { Passa } \\
\text { Passa } \\
\text { Passa } \\
\text { Passa }\end{array}$ \\
\hline $\begin{array}{l}\text { Todas as verificações foram } \\
\text { Referência: P14 } \\
\text { Dimensões: } 90 \times 90 \times 30 / 20 \\
\text { Soldados: Xi: Ø10c/28 Yi: Ø10c/28 }\end{array}$ & cumpridas & \\
\hline Verificação & Valores & Estado \\
\hline $\begin{array}{l}\text { Ângulo máximo talude: } \\
\text { Critério da CYPE Ingenieros }\end{array}$ & $\begin{array}{l}\text { Máximo: } 30 \text { graus } \\
\text { Calculado: } 18.4349 \text { graus }\end{array}$ & Passa \\
\hline $\begin{array}{l}\text { Tensões sobre o terreno: } \\
\text { Critério da CYPE Ingenieros } \\
\text { - Tensão média em combinações fundamentais: } \\
\text { - Tensão máxima em combinações fundamentais: }\end{array}$ & $\begin{array}{l}\text { Máximo: } 0.24525 \mathrm{MPa} \\
\text { Calculado: } 0.0770085 \mathrm{MPa} \\
\text { Máximo: } 0.306563 \mathrm{MPa} \\
\text { Calculado: } 0.183153 \mathrm{MPa}\end{array}$ & $\begin{array}{l}\text { Passa } \\
\text { Passa }\end{array}$ \\
\hline $\begin{array}{l}\text { Tombamento da sapata: } \\
\text { se o \% de reserva de segurança é maior que zero, pode ser dito que } \\
\text { os coeficientes de segurança ao tombamento são maiores que os } \\
\text { valores exatos exigidos para todas as combinaçôes de equilíbrio. } \\
\text { - Na direção X: } \\
\text { - Na direção Y: }\end{array}$ & $\begin{array}{l}\text { Reserva segurança: } 259.3 \% \\
\text { Reserva segurança: } 287.8 \%\end{array}$ & $\begin{array}{l}\text { Passa } \\
\text { Passa }\end{array}$ \\
\hline $\begin{array}{l}\text { Flexão na sapata: } \\
\text { - Na direção X: } \\
\text { - Na direção Y: }\end{array}$ & $\begin{array}{l}\text { Momento: } 9.67 \mathrm{kN} \cdot \mathrm{m} \\
\text { Momento: } 9.46 \mathrm{kN} \cdot \mathrm{m}\end{array}$ & $\begin{array}{l}\text { Passa } \\
\text { Passa }\end{array}$ \\
\hline & & \\
\hline
\end{tabular}




\begin{tabular}{|c|c|c|}
\hline \multicolumn{3}{|l|}{$\begin{array}{l}\text { Referência: P14 } \\
\text { Dimensões: } 90 \text { × } 90 \text { × } 30 / 20 \\
\text { Soldados: Xi: Ø10c/28 Yi:Ø10c/28 }\end{array}$} \\
\hline Verificação & Valores & Estado \\
\hline $\begin{array}{l}\text { - Na direção X: } \\
\text { - Na direção Y: }\end{array}$ & $\begin{array}{l}\text { Cortante: } 14.72 \mathrm{kN} \\
\text { Cortante: } 14.32 \mathrm{kN}\end{array}$ & $\begin{array}{l}\text { Passa } \\
\text { Passa }\end{array}$ \\
\hline $\begin{array}{l}\text { Compressão oblíqua na sapata: } \\
\text { - Combinações fundamentais: } \\
\text { Critério da CYPE Ingenieros }\end{array}$ & $\begin{array}{l}\text { Máximo: } 5844.1 \mathrm{kN} / \mathrm{m}^{2} \\
\text { Calculado: } 428.8 \mathrm{kN} / \mathrm{m}^{2}\end{array}$ & Passa \\
\hline $\begin{array}{l}\text { Altura mínima: } \\
\text { Critério da CYPE Ingenieros }\end{array}$ & $\begin{array}{l}\text { Mínimo: } 15 \mathrm{~cm} \\
\text { Calculado: } 20 \mathrm{~cm}\end{array}$ & Passa \\
\hline $\begin{array}{l}\text { Espaço para ancorar arranques na fundação: } \\
\text { - P14: }\end{array}$ & $\begin{array}{l}\text { Mínimo: } 12 \mathrm{~cm} \\
\text { Calculado: } 24 \mathrm{~cm}\end{array}$ & Passa \\
\hline $\begin{array}{l}\text { Quantidade geométrica mínima: } \\
\text { Critério da CYPE Ingenieros } \\
\text { - Armadura inferior direção X: } \\
\text { - Armadura inferior direção Y: }\end{array}$ & $\begin{array}{l}\text { Mínimo: } 0.001 \\
\text { Calculado: } 0.001 \\
\text { Calculado: } 0.001\end{array}$ & $\begin{array}{l}\text { Passa } \\
\text { Passa }\end{array}$ \\
\hline $\begin{array}{l}\text { Quantia mínima necessária por flexão: } \\
\text { Norma Brasileira ABNT NBR 6118:2014. Artigo 17.3.5.2 } \\
\text { - Armadura inferior direção X: } \\
\text { - Armadura inferior direção Y: }\end{array}$ & $\begin{array}{l}\text { Mínimo: } 0.0005 \\
\text { Calculado: } 0.0011 \\
\text { Calculado: } 0.0011\end{array}$ & $\begin{array}{l}\text { Passa } \\
\text { Passa }\end{array}$ \\
\hline $\begin{array}{l}\text { Diâmetro mínimo das barras: } \\
\text { - Malha inferior: } \\
\text { Critério da CYPE Ingenieros }\end{array}$ & $\begin{array}{l}\text { Mínimo: } 10 \mathrm{~mm} \\
\text { Calculado: } 10 \mathrm{~mm}\end{array}$ & Passa \\
\hline $\begin{array}{l}\text { Espaçamento máximo entre barras: } \\
\text { Critério da CYPE Ingenieros } \\
\text { - Armadura inferior direção } X: \\
\text { - Armadura inferior direção } Y \text { : }\end{array}$ & $\begin{array}{l}\text { Máximo: } 30 \mathrm{~cm} \\
\text { Calculado: } 28 \mathrm{~cm} \\
\text { Calculado: } 28 \mathrm{~cm}\end{array}$ & $\begin{array}{l}\text { Passa } \\
\text { Passa }\end{array}$ \\
\hline $\begin{array}{l}\text { Espaçamento mínimo entre barras: } \\
\text { Critério da CYPE Ingenieros, baseado em: J. Calavera. "Cálculo de } \\
\text { Estructuras de Cimentación". Capitulo 3.16 } \\
\text { - Armadura inferior direção X: } \\
\text { - Armadura inferior direção Y: }\end{array}$ & $\begin{array}{l}\text { Mínimo: } 10 \mathrm{~cm} \\
\text { Calculado: } 28 \mathrm{~cm} \\
\text { Calculado: } 28 \mathrm{~cm}\end{array}$ & $\begin{array}{l}\text { Passa } \\
\text { Passa }\end{array}$ \\
\hline $\begin{array}{l}\text { Comprimento de ancoragem: } \\
\text { Critério do livro "Célculo de estructuras de cimentación", J. Calavera. } \\
\text { Ed. INTEMAC, } 1991 \\
\text { - Armadura inf. direção X para dir: } \\
\text { - Armadura inf. direção X para esq: } \\
\text { - Armadura inf. direção Y para cima: } \\
\text { - Armadura inf. direção Y para baixo: }\end{array}$ & $\begin{array}{l}\text { Calculado: } 32 \mathrm{~cm} \\
\text { Mínimo: } 11 \mathrm{~cm} \\
\text { Mínimo: } 22 \mathrm{~cm} \\
\text { Mínimo: } 11 \mathrm{~cm} \\
\text { Mínimo: } 22 \mathrm{~cm}\end{array}$ & $\begin{array}{l}\text { Passa } \\
\text { Passa } \\
\text { Passa } \\
\text { Passa }\end{array}$ \\
\hline Comprimento mínimo das dobras: & Mínimo: $11 \mathrm{~cm}$ & \\
\hline
\end{tabular}




\begin{tabular}{|c|c|c|}
\hline $\begin{array}{l}\text { Referência: P14 } \\
\text { Dimensões: } 90 \text { × } 90 \text { × } 30 / 20 \\
\text { Soldados: Xi: Ø10c/28 Yi:Ø10c/28 }\end{array}$ & & \\
\hline Verificação & Valores & Estado \\
\hline $\begin{array}{l}\text { - Armadura inf. direção X para dir: } \\
\text { - Armadura inf. direção X para esq: } \\
\text { - Armadura inf. direção Y para cima: } \\
\text { - Armadura inf. direção Y para baixo: }\end{array}$ & $\begin{array}{l}\text { Calculado: } 21 \mathrm{~cm} \\
\text { Calculado: } 21 \mathrm{~cm} \\
\text { Calculado: } 21 \mathrm{~cm} \\
\text { Calculado: } 21 \mathrm{~cm}\end{array}$ & $\begin{array}{l}\text { Passa } \\
\text { Passa } \\
\text { Passa } \\
\text { Passa }\end{array}$ \\
\hline $\begin{array}{l}\text { Todas as verificações foran } \\
\text { Referência: P15 } \\
\text { Dimensões: } 95 \times 95 \times 30 / 20 \\
\text { Soldados: Xi: Ø10c/28 Yi:Ø10c/28 }\end{array}$ & cumpridas & \\
\hline Verificação & Valores & Estado \\
\hline $\begin{array}{l}\text { Ângulo máximo talude: } \\
\text { Critério da CYPE Ingenieros }\end{array}$ & $\begin{array}{l}\text { Máximo: } 30 \text { graus } \\
\text { Calculado: } 18.4349 \text { graus }\end{array}$ & Passa \\
\hline $\begin{array}{l}\text { Tensões sobre o terreno: } \\
\text { Critério da CYPE Ingenieros } \\
\text { - Tensão média em combinações fundamentais: } \\
\text { - Tensão máxima em combinações fundamentais: }\end{array}$ & $\begin{array}{l}\text { Máximo: } 0.24525 \mathrm{MPa} \\
\text { Calculado: } 0.149406 \mathrm{MPa} \\
\text { Máximo: } 0.306563 \mathrm{MPa} \\
\text { Calculado: } 0.265459 \mathrm{MPa}\end{array}$ & $\begin{array}{l}\text { Passa } \\
\text { Passa }\end{array}$ \\
\hline $\begin{array}{l}\text { Tombamento da sapata: } \\
\text { Se o \% de reserva de segurança é maior que zero, pode ser dito que } \\
\text { os coeficientes de segurança ao tombamento são maiores que os } \\
\text { valores exatos exigidos para todas as combinações de equilíbrio. } \\
\text { - Na direção X: } \\
\text { - Na direção Y: }\end{array}$ & $\begin{array}{l}\text { Reserva segurança: } 817.0 \% \\
\text { Reserva segurança: } 453.2 \%\end{array}$ & $\begin{array}{l}\text { Passa } \\
\text { Passa }\end{array}$ \\
\hline $\begin{array}{l}\text { Flexão na sapata: } \\
\text { - Na direção X: } \\
\text { - Na direção Y: }\end{array}$ & $\begin{array}{l}\text { Momento: } 17.48 \mathrm{kN} \cdot \mathrm{m} \\
\text { Momento: } 21.29 \mathrm{kN} \cdot \mathrm{m}\end{array}$ & $\begin{array}{l}\text { Passa } \\
\text { Passa }\end{array}$ \\
\hline $\begin{array}{l}\text { Cortante na sapata: } \\
\text { - Na direção X: } \\
\text { - Na direção Y: }\end{array}$ & $\begin{array}{l}\text { Cortante: } 24.33 \mathrm{kN} \\
\text { Cortante: } 34.34 \mathrm{kN}\end{array}$ & $\begin{array}{l}\text { Passa } \\
\text { Passa }\end{array}$ \\
\hline $\begin{array}{l}\text { Compressão oblíqua na sapata: } \\
\text { - Combinações fundamentais: } \\
\text { Critério da CYPE Ingenieros }\end{array}$ & $\begin{array}{l}\text { Máximo: } 5844.1 \mathrm{kN} / \mathrm{m}^{2} \\
\text { Calculado: } 925.5 \mathrm{kN} / \mathrm{m}^{2}\end{array}$ & Passa \\
\hline $\begin{array}{l}\text { Altura mínima: } \\
\text { Critério da CYPE Ingenieros }\end{array}$ & $\begin{array}{l}\text { Mínimo: } 15 \mathrm{~cm} \\
\text { Calculado: } 20 \mathrm{~cm}\end{array}$ & Passa \\
\hline $\begin{array}{l}\text { Espaço para ancorar arranques na fundação: } \\
\text { - P15: }\end{array}$ & $\begin{array}{l}\text { Mínimo: } 12 \mathrm{~cm} \\
\text { Calculado: } 24 \mathrm{~cm}\end{array}$ & Passa \\
\hline $\begin{array}{l}\text { Quantidade geométrica mínima: } \\
\text { Critério da CYPE Ingenieros } \\
\text { - Armadura inferior direção X: }\end{array}$ & $\begin{array}{l}\text { Mínimo: } 0.001 \\
\text { Calculado: } 0.001\end{array}$ & Passa \\
\hline
\end{tabular}




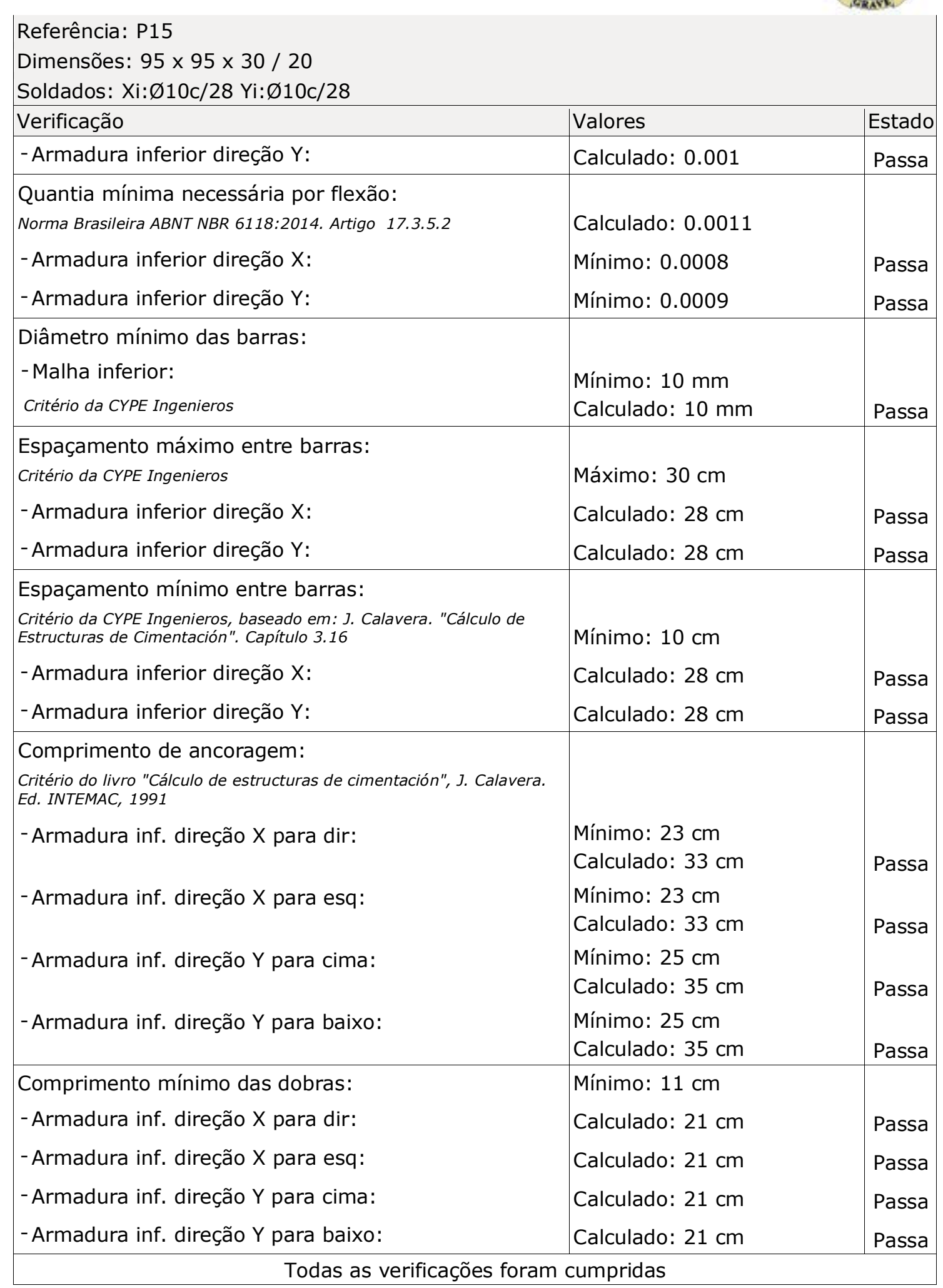




\begin{tabular}{|c|c|c|}
\hline $\begin{array}{l}\text { Referência: P16 } \\
\text { Dimensões: } 90 \text { × } 90 \text { × } 30 / 20 \\
\text { Soldados: Xi: } \varnothing 10 c / 28 \text { Yi: Ø10c/28 }\end{array}$ & & \\
\hline Verificação & Valores & Estado \\
\hline $\begin{array}{l}\text { Ângulo máximo talude: } \\
\text { Critério da CYPE Ingenieros }\end{array}$ & $\begin{array}{l}\text { Máximo: } 30 \text { graus } \\
\text { Calculado: } 18.4349 \text { graus }\end{array}$ & Passa \\
\hline $\begin{array}{l}\text { Tensões sobre o terreno: } \\
\text { Critério da CYPE Ingenieros } \\
\text {-Tensão média em combinações fundamentais: } \\
\text {-Tensão máxima em combinações fundamentais: }\end{array}$ & $\begin{array}{l}\text { Máximo: } 0.24525 \mathrm{MPa} \\
\text { Calculado: } 0.086328 \mathrm{MPa} \\
\text { Máximo: } 0.306563 \mathrm{MPa} \\
\text { Calculado: } 0.202478 \mathrm{MPa}\end{array}$ & $\begin{array}{l}\text { Passa } \\
\text { Passa }\end{array}$ \\
\hline $\begin{array}{l}\text { Tombamento da sapata: } \\
\text { Se o \% de reserva de segurança é maior que zero, pode ser dito que } \\
\text { os coeficientes de segurança ao tombamento são maiores que os } \\
\text { valores exatos exigidos para todas as combinaçôes de equilibrio. } \\
\text { - Na direção X: } \\
\text { - Na direção Y: }\end{array}$ & $\begin{array}{l}\text { Reserva segurança: } 229.5 \% \\
\text { Reserva segurança: } 336.6 \%\end{array}$ & $\begin{array}{l}\text { Passa } \\
\text { Passa }\end{array}$ \\
\hline $\begin{array}{l}\text { Flexão na sapata: } \\
\text { - Na direção X: } \\
\text { - Na direção Y: }\end{array}$ & $\begin{array}{l}\text { Momento: } 11.27 \mathrm{kN} \cdot \mathrm{m} \\
\text { Momento: } 10.24 \mathrm{kN} \cdot \mathrm{m}\end{array}$ & $\begin{array}{l}\text { Passa } \\
\text { Passa }\end{array}$ \\
\hline $\begin{array}{l}\text { Cortante na sapata: } \\
\text { - Na direção X: } \\
\text { - Na direção Y: }\end{array}$ & $\begin{array}{l}\text { Cortante: } 17.17 \mathrm{kN} \\
\text { Cortante: } 15.40 \mathrm{kN}\end{array}$ & $\begin{array}{l}\text { Passa } \\
\text { Passa }\end{array}$ \\
\hline $\begin{array}{l}\text { Compressão oblíqua na sapata: } \\
\text { - Combinações fundamentais: } \\
\text { Critério da CYPE Ingenieros }\end{array}$ & $\begin{array}{l}\text { Máximo: } 5844.1 \mathrm{kN} / \mathrm{m}^{2} \\
\text { Calculado: } 490.1 \mathrm{kN} / \mathrm{m}^{2}\end{array}$ & Passa \\
\hline $\begin{array}{l}\text { Altura mínima: } \\
\text { Critério da CYPE Ingenieros }\end{array}$ & $\begin{array}{l}\text { Mínimo: } 15 \mathrm{~cm} \\
\text { Calculado: } 20 \mathrm{~cm}\end{array}$ & Passa \\
\hline $\begin{array}{l}\text { Espaço para ancorar arranques na fundação: } \\
\text { - P16: }\end{array}$ & $\begin{array}{l}\text { Mínimo: } 12 \mathrm{~cm} \\
\text { Calculado: } 24 \mathrm{~cm}\end{array}$ & Passa \\
\hline $\begin{array}{l}\text { Quantidade geométrica mínima: } \\
\text { Critério da CYPE Ingenieros } \\
\text { - Armadura inferior direção X: } \\
\text { - Armadura inferior direção Y: }\end{array}$ & $\begin{array}{l}\text { Mínimo: } 0.001 \\
\text { Calculado: } 0.001 \\
\text { Calculado: } 0.001\end{array}$ & $\begin{array}{l}\text { Passa } \\
\text { Passa }\end{array}$ \\
\hline $\begin{array}{l}\text { Quantia mínima necessária por flexão: } \\
\text { Norma Brasileira ABNT NBR 6118:2014. Artigo 17.3.5.2 } \\
\text { - Armadura inferior direção X: } \\
\text { - Armadura inferior direção Y: }\end{array}$ & $\begin{array}{l}\text { Mínimo: } 0.0005 \\
\text { Calculado: } 0.0011 \\
\text { Calculado: } 0.0011\end{array}$ & $\begin{array}{l}\text { Passa } \\
\text { Passa }\end{array}$ \\
\hline $\begin{array}{l}\text { Diâmetro mínimo das barras: } \\
\text { - Malha inferior: } \\
\text { Critério da CYPE Ingenieros }\end{array}$ & $\begin{array}{l}\text { Mínimo: } 10 \mathrm{~mm} \\
\text { Calculado: } 10 \mathrm{~mm}\end{array}$ & Passa \\
\hline
\end{tabular}




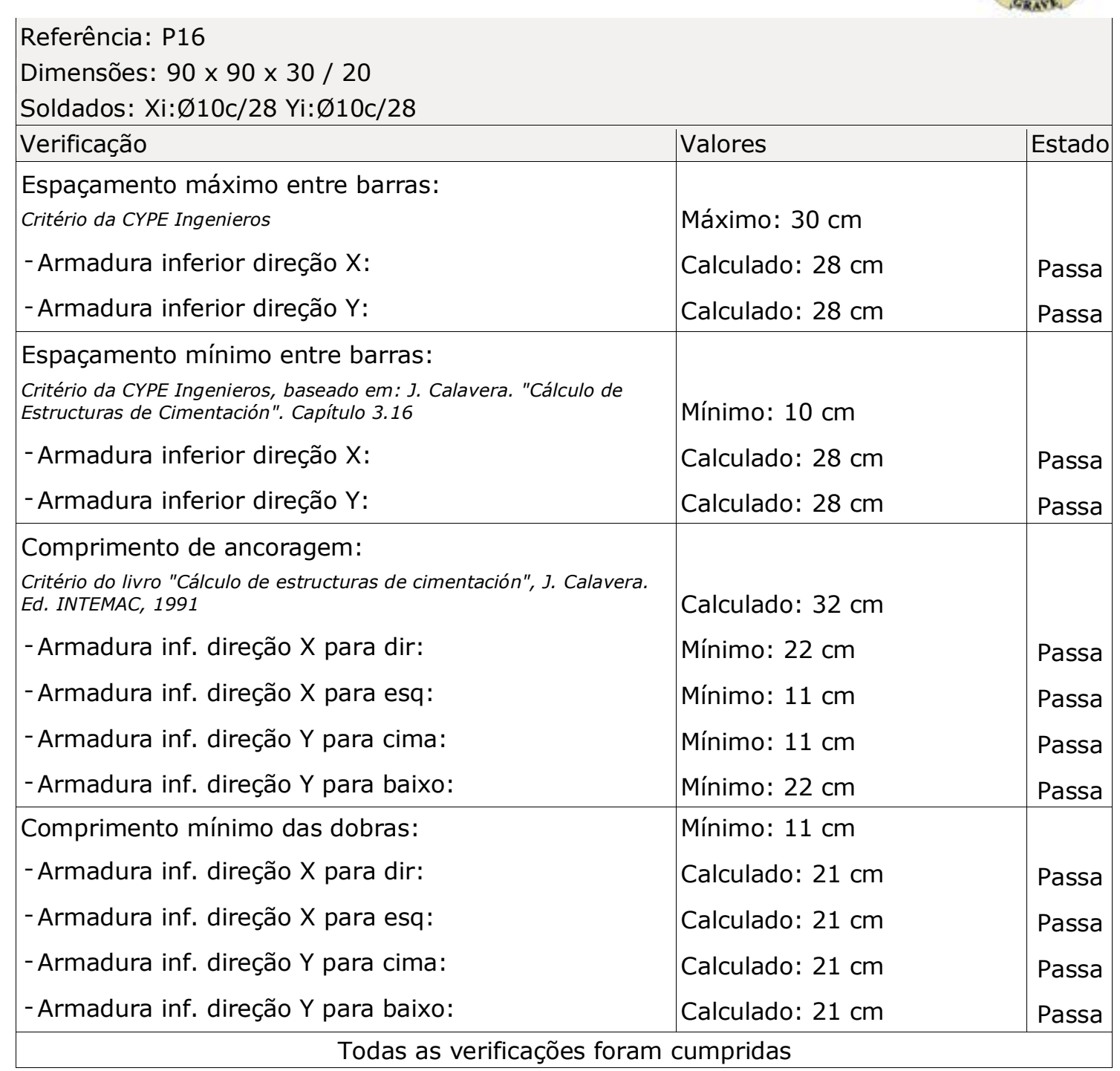

\subsection{4.- VERIFICAÇÃO DOS PILARES}

\section{P1}

\begin{tabular}{|c|c|c|c|c|c|c|c|c|c|c|c|c|c|c|c|}
\hline \multicolumn{16}{|c|}{ Secção de betão } \\
\hline \multirow[b]{2}{*}{ Tramo } & \multirow[b]{2}{*}{$\begin{array}{c}\text { Dimens } \\
\text { ão } \\
(\mathrm{cm})\end{array}$} & \multirow[b]{2}{*}{ Posição } & \multicolumn{5}{|c|}{ Verificações } & \multicolumn{7}{|c|}{ Esforços desfavoráveis } & \multirow[b]{2}{*}{$\begin{array}{c}\text { Estad } \\
0\end{array}$} \\
\hline & & & Disp. & Arm. & $\begin{array}{c}\mathrm{Q} \\
(\% \\
)\end{array}$ & $\begin{array}{c}\mathrm{N}, \\
\mathrm{M} \\
(\% \\
)\end{array}$ & $\begin{array}{c}\text { Apro } \\
\text { V. } \\
(\%)\end{array}$ & $\begin{array}{c}\text { Naturez } \\
\text { a }\end{array}$ & Verif & $\begin{array}{c}\mathrm{N} \\
(\mathrm{kN} \\
)\end{array}$ & $\begin{array}{c}M x x \\
(k N . \\
m)\end{array}$ & $\begin{array}{c}\text { Myy } \\
(\mathrm{kN} . \\
\mathrm{m})\end{array}$ & $\begin{array}{l}\text { Qx } \\
(k N)\end{array}$ & $\begin{array}{c}\text { Qy } \\
(\mathrm{kN})\end{array}$ & \\
\hline \multirow{2}{*}{$\begin{array}{l}\text { Cobertura }(0-3.15 \\
\mathrm{m})\end{array}$} & \multirow{2}{*}{$20 \times 20$} & Ext.Superior & $\begin{array}{l}\text { Pass } \\
\text { a }\end{array}$ & $\begin{array}{l}\text { Pass } \\
\text { a }\end{array}$ & 2.6 & 8.7 & 8.7 & $\begin{array}{l}\mathrm{AP}, \\
\mathrm{SCU}(2)\end{array}$ & $\begin{array}{l}\mathrm{Q}, \mathrm{N}, \\
\mathrm{M}\end{array}$ & $\begin{array}{l}31 . \\
5\end{array}$ & -0.8 & 0.3 & 0.2 & -0.9 & Passa \\
\hline & & Ext.Inferior & \begin{tabular}{|l|} 
Pass \\
a
\end{tabular} & $\begin{array}{l}\text { Pass } \\
a\end{array}$ & 3.5 & $\begin{array}{l}12 . \\
7\end{array}$ & 12.7 & $\begin{array}{l}\mathrm{AP}, \\
\mathrm{SCU}(2)\end{array}$ & $\begin{array}{l}\text { Q,N, } \\
M\end{array}$ & $\begin{array}{l}35 . \\
1\end{array}$ & 1.5 & 0.8 & 0.2 & -0.9 & Passa \\
\hline \multirow{2}{*}{$\begin{array}{l}\text { Cintamento }(-1-0 \\
\mathrm{m})\end{array}$} & \multirow{2}{*}{$20 \times 20$} & Ext.Superior & \begin{tabular}{|l|} 
Pass \\
a
\end{tabular} & $\begin{array}{l}\text { Pass } \\
\mathrm{a}\end{array}$ & $\begin{array}{l}53 . \\
3\end{array}$ & $\begin{array}{l}42 . \\
0\end{array}$ & 53.3 & $\begin{array}{l}\text { AP, } \\
S C U^{(2)}\end{array}$ & $\begin{array}{l}Q, N, \\
M\end{array}$ & $\begin{array}{l}95 . \\
0\end{array}$ & -6.9 & 6.6 & - & - & Passa \\
\hline & & Ext.Inferior & \begin{tabular}{|l|} 
Pass \\
a
\end{tabular} & $\begin{array}{l}\text { Pass } \\
\mathrm{a}\end{array}$ & $\begin{array}{l}53 . \\
3\end{array}$ & $\begin{array}{l}31 . \\
6 \\
\end{array}$ & 53.3 & $\begin{array}{l}\mathrm{AP}, \\
\mathrm{SCU}^{(2)}\end{array}$ & $\begin{array}{l}\mathrm{Q}, \mathrm{N}, \\
\mathrm{M}\end{array}$ & $\begin{array}{l}95 . \\
7\end{array}$ & 5.3 & -4.8 & - & 27.1 & Passa \\
\hline Fundação & $20 \times 20$ & $\begin{array}{l}\text { Elemento de } \\
\text { Fundação }\end{array}$ & $\begin{array}{l}\text { N.P. } \\
1)\end{array}$ & $\begin{array}{l}\text { N.P.' } \\
1)\end{array}$ & 24. & $\begin{array}{l}31 . \\
6\end{array}$ & 31.6 & $\begin{array}{l}\mathrm{AP}, \\
\mathrm{SCU}{ }^{(2)}\end{array}$ & $\begin{array}{l}\mathrm{Q}, \mathrm{N}, \\
\mathrm{M}\end{array}$ & $\begin{array}{l}95 . \\
7\end{array}$ & 5.3 & -4.8 & $-\overline{25.3}$ & $-\overline{27.1}$ & Passa \\
\hline
\end{tabular}




\section{Pontifícia Universidade Católlca}

DO RIO DE JANEIRO

\begin{tabular}{|c|c|c|c|c|c|c|c|c|c|c|c|c|c|c|c|}
\hline \multicolumn{16}{|c|}{ Secção de betão } \\
\hline \multirow[b]{2}{*}{ Tramo } & \multirow[b]{2}{*}{$\begin{array}{c}\text { Dimens } \\
\text { ão } \\
(\mathrm{cm})\end{array}$} & \multirow[b]{2}{*}{ Posição } & \multicolumn{5}{|c|}{ Verificações } & \multicolumn{7}{|c|}{ Esforços desfavoráveis } & \multirow[b]{2}{*}{$\begin{array}{c}\text { Estad } \\
0\end{array}$} \\
\hline & & & Disp. & Arm. & $\begin{array}{c}\mathrm{Q} \\
(\% \\
)\end{array}$ & $\begin{array}{c}\mathrm{N} \\
\mathrm{M} \\
(\% \\
)\end{array}$ & $\begin{array}{c}\text { Apro } \\
\text { V. } \\
(\%)\end{array}$ & $\begin{array}{c}\text { Naturez } \\
a\end{array}$ & $\begin{array}{c}\text { Verif } \\
.\end{array}$ & $\begin{array}{c}\mathrm{N} \\
(\mathrm{kN} \\
)\end{array}$ & $\begin{array}{l}M x x \\
(k N . \\
m)\end{array}$ & $\begin{array}{l}\text { Myy } \\
(\mathrm{kN} . \\
\mathrm{m})\end{array}$ & $\begin{array}{l}\text { Qx } \\
(\mathrm{kN})\end{array}$ & $\begin{array}{c}\text { Qy } \\
(k N)\end{array}$ & \\
\hline $\begin{array}{l}\text { Notas: } \\
\text { (1) A verifica } \\
\text { (2) } 1.4 \cdot P P+1\end{array}$ & $\begin{array}{l}\text { necessár } \\
\text {.Qa }\end{array}$ & & & & & & & & & & & & & & \\
\hline
\end{tabular}

P2

\begin{tabular}{|c|c|c|c|c|c|c|c|c|c|c|c|c|c|c|c|}
\hline \multicolumn{16}{|c|}{ Secção de betão } \\
\hline \multirow[b]{2}{*}{ Tramo } & \multirow[b]{2}{*}{$\begin{array}{c}\text { Dimens } \\
\text { ão } \\
(\mathrm{cm})\end{array}$} & \multirow[b]{2}{*}{ Posição } & \multicolumn{5}{|c|}{ Verificações } & \multicolumn{7}{|c|}{ Esforços desfavoráveis } & \multirow[b]{2}{*}{$\begin{array}{c}\text { Estad } \\
0\end{array}$} \\
\hline & & & Disp. & Arm. & $\begin{array}{c}\mathrm{Q} \\
(\% \\
)\end{array}$ & \begin{tabular}{|c|}
$\mathrm{N}$ \\
$\mathrm{M}$ \\
$(\%$ \\
)
\end{tabular} & $\begin{array}{c}\text { Apro } \\
\text { V. } \\
(\%)\end{array}$ & $\begin{array}{c}\text { Naturez } \\
\text { a }\end{array}$ & $\begin{array}{c}\text { Verif } \\
.\end{array}$ & $\begin{array}{c}\mathrm{N} \\
(\mathrm{kN})\end{array}$ & $\begin{array}{l}\mathrm{Mxx} \\
(\mathrm{kN} \cdot \\
\mathrm{m})\end{array}$ & $\begin{array}{l}\text { Myy } \\
(\mathrm{kN} \text {. } \\
\mathrm{m})\end{array}$ & $\begin{array}{c}\text { Qx } \\
(\mathrm{kN} \\
)\end{array}$ & $\begin{array}{c}\text { Qy } \\
(\mathrm{kN} \\
)\end{array}$ & \\
\hline \multirow{2}{*}{$\begin{array}{l}\text { Cobertura (0 - } 3.15 \\
\mathrm{~m})\end{array}$} & \multirow{2}{*}{$20 \times 20$} & Ext.Superior & $\begin{array}{l}\text { Pass } \\
\text { a }\end{array}$ & $\begin{array}{l}\text { Pass } \\
a\end{array}$ & 3.8 & $\begin{array}{l}19 . \\
9\end{array}$ & 19.9 & $\begin{array}{l}\mathrm{AP}, \\
\mathrm{SCU}^{(2)}\end{array}$ & $\begin{array}{l}\mathrm{Q}, \mathrm{N}, \\
\mathrm{M}\end{array}$ & 82.1 & -0.1 & -1.5 & 1.8 & $\overline{-}$ & Passa \\
\hline & & Ext.Inferior & $\begin{array}{l}\text { Pass } \\
\text { a }\end{array}$ & $\begin{array}{l}\text { Pass } \\
\text { a }\end{array}$ & 6.9 & $\begin{array}{l}24 . \\
7\end{array}$ & 24.7 & $\begin{array}{l}\mathrm{AP}_{1} \\
\mathrm{SCU}^{(2)}\end{array}$ & $\begin{array}{l}\mathrm{Q}_{,} \mathrm{N}, \\
\mathrm{M}\end{array}$ & 85.6 & 0.2 & 3.1 & 1.8 & $-\overline{0}$ & Passa \\
\hline \multirow{4}{*}{$\begin{array}{l}\text { Cintamento (-1 - } 0 \\
\mathrm{~m})\end{array}$} & \multirow{4}{*}{$20 \times 20$} & \multirow{2}{*}{ Ext.Superior } & \multirow{2}{*}{$\begin{array}{l}\text { Pass } \\
\text { a }\end{array}$} & \multirow{2}{*}{$\begin{array}{l}\text { Pass } \\
a\end{array}$} & \multirow{2}{*}{$\begin{array}{l}47 . \\
8\end{array}$} & \multirow{2}{*}{$\begin{array}{l}24 . \\
4\end{array}$} & \multirow{2}{*}{47.8} & $\mathrm{AP}^{(3)}$ & Q & $\begin{array}{l}122 . \\
3\end{array}$ & -2.1 & -3.5 & $\begin{array}{l}13 . \\
4\end{array}$ & 8.4 & \multirow{2}{*}{ Passa } \\
\hline & & & & & & & & $\begin{array}{l}\mathrm{AP}, \\
\mathrm{SCU}^{(2)}\end{array}$ & $\mathrm{N}, \mathrm{M}$ & $\begin{array}{ll}143 . \\
0\end{array}$ & -2.2 & -3.5 & $\begin{array}{ll}13 . \\
4\end{array}$ & 8.7 & \\
\hline & & \multirow{2}{*}{ Ext.Inferior } & \multirow{2}{*}{$\begin{array}{l}\text { Pass } \\
\text { a }\end{array}$} & \multirow{2}{*}{$\begin{array}{l}\text { Pass } \\
\text { a }\end{array}$} & \multirow{2}{*}{$\begin{array}{l}36 . \\
2\end{array}$} & \multirow{2}{*}{$\begin{array}{l}24 . \\
1\end{array}$} & \multirow{2}{*}{36.2} & $\mathrm{AP}^{(3)}$ & Q & $\begin{array}{l}122 . \\
9\end{array}$ & 1.7 & 2.5 & \begin{tabular}{|l|}
13. \\
4
\end{tabular} & $\overline{8.4}$ & \multirow{2}{*}{ Passa } \\
\hline & & & & & & & & $\begin{array}{l}\mathrm{AP}_{1} \\
\mathrm{SCU}^{(2)}\end{array}$ & $N, M$ & $\begin{array}{l}143 . \\
6\end{array}$ & 1.7 & 2.6 & \begin{tabular}{l|}
13. \\
4
\end{tabular} & 8.7 & \\
\hline Fundação & $20 \times 20$ & $\begin{array}{l}\text { Elemento de } \\
\text { Fundação }\end{array}$ & $\begin{array}{l}\text { N.P. } \\
1)\end{array}$ & $\begin{array}{l}\text { N.P. } \\
1)\end{array}$ & $\begin{array}{l}10 . \\
6\end{array}$ & $\begin{array}{l}24 . \\
1\end{array}$ & 24.1 & $\begin{array}{l}\mathrm{AP}, \\
\mathrm{SCU}^{(2)}\end{array}$ & $\begin{array}{l}Q, N, \\
M\end{array}$ & $\begin{array}{l}143 . \\
6\end{array}$ & 1.7 & 2.6 & $\begin{array}{l}13 . \\
4\end{array}$ & 8.7 & Passa \\
\hline \multicolumn{16}{|c|}{$\begin{array}{l}\text { Notas: } \\
\text { (1) } A \text { verificação não é necessária } \\
\text { (2) } 1.4 \cdot P P+1.4 \cdot C P+1.4 \cdot Q a \\
\text { (3) } 1.4 \cdot P P+1.4 \cdot C P\end{array}$} \\
\hline
\end{tabular}

P3

\begin{tabular}{|c|c|c|c|c|c|c|c|c|c|c|c|c|c|c|c|}
\hline \multicolumn{16}{|c|}{ Secção de betão } \\
\hline \multirow[b]{2}{*}{ Tramo } & \multirow[b]{2}{*}{$\begin{array}{c}\text { Dimens } \\
\text { ão } \\
(\mathrm{cm})\end{array}$} & \multirow[b]{2}{*}{ Posição } & \multicolumn{5}{|c|}{ Verificações } & \multicolumn{7}{|c|}{ Esforços desfavoráveis } & \multirow[b]{2}{*}{$\begin{array}{c}\text { Estad } \\
0\end{array}$} \\
\hline & & & Disp. & Arm. & $\begin{array}{c}Q \\
(\% \\
) \\
\end{array}$ & $\begin{array}{c}\mathrm{N}, \mathrm{M} \\
(\% \\
)\end{array}$ & $\begin{array}{c}\text { Apro } \\
\text { V. } \\
(\%) \\
\end{array}$ & $\begin{array}{c}\text { Naturez } \\
a\end{array}$ & Verif. & $\begin{array}{c}\mathrm{N} \\
(\mathrm{kN} \\
)\end{array}$ & $\begin{array}{c}M \times x \\
(k N \cdot \\
m)\end{array}$ & $\begin{array}{c}\text { Myy } \\
(\mathrm{kN} . \\
\mathrm{m})\end{array}$ & $\begin{array}{c}\text { Qx } \\
(\mathrm{kN} \\
) \\
\end{array}$ & \begin{tabular}{|c|} 
Qy \\
$(\mathrm{kN}$ \\
) \\
\end{tabular} & \\
\hline \multirow{2}{*}{$\begin{array}{l}\text { Cobertura }(0-3.15 \\
\mathrm{m})\end{array}$} & \multirow{2}{*}{$20 \times 20$} & Ext.Superior & \begin{tabular}{|l|} 
Pass \\
a
\end{tabular} & \begin{tabular}{|l|} 
Pass \\
a
\end{tabular} & 7.8 & $\begin{array}{l}10 . \\
5\end{array}$ & 10.5 & $\begin{array}{l}\mathrm{AP} \\
\mathrm{SCU}^{(2)}\end{array}$ & $\begin{array}{l}\mathrm{Q}, \mathrm{N}, \\
\mathrm{M}\end{array}$ & $\begin{array}{l}23 . \\
2\end{array}$ & 0.2 & -1.9 & 2.0 & 0.3 & Passa \\
\hline & & Ext.Inferior & \begin{tabular}{|l|} 
Pass \\
a
\end{tabular} & \begin{tabular}{|l|} 
Pass \\
a
\end{tabular} & 7.8 & $\begin{array}{l}17 . \\
9\end{array}$ & 17.9 & $\begin{array}{l}\mathrm{AP} \\
\mathrm{SCU}^{(2)}\end{array}$ & $\begin{array}{l}\mathrm{Q}, \mathrm{N}, \\
\mathrm{M}\end{array}$ & $\begin{array}{l}26 . \\
7\end{array}$ & -0.6 & 3.3 & 2.0 & 0.3 & Passa \\
\hline \multirow{3}{*}{$\begin{array}{l}\text { Cintamento }(-1-0 \\
\mathrm{m})\end{array}$} & \multirow{3}{*}{$20 \times 20$} & $0 \mathrm{~m}$ & $\begin{array}{l}\text { N.P. } \\
1)\end{array}$ & $\begin{array}{l}\text { N.P. ( } \\
1)\end{array}$ & 1.3 & $\begin{array}{l}17 . \\
9\end{array}$ & 17.9 & $\begin{array}{l}\mathrm{AP} \\
\mathrm{SCU}(2)\end{array}$ & $\begin{array}{l}\mathrm{Q}_{1} \mathrm{~N}, \\
\mathrm{M}\end{array}$ & $\begin{array}{l}26 . \\
7\end{array}$ & -0.6 & 3.3 & 2.0 & 0.3 & Passa \\
\hline & & Ext.Superior & \begin{tabular}{|l|} 
Pass \\
a
\end{tabular} & $\begin{array}{l}\text { Pass } \\
\text { a }\end{array}$ & 5.5 & 5.9 & 5.9 & $\begin{array}{l}\mathrm{AP} \\
\mathrm{SCU}^{(2)}\end{array}$ & $\begin{array}{l}\mathrm{Q}, \mathrm{N}_{1} \\
\mathrm{M}\end{array}$ & $\begin{array}{l}37 . \\
8\end{array}$ & -0.4 & -0.6 & 2.5 & - & Passa \\
\hline & & Ext.Inferior & \begin{tabular}{|l|} 
Pass \\
a
\end{tabular} & $\begin{array}{l}\text { Pass } \\
\text { a }\end{array}$ & 5.5 & 6.1 & 6.1 & $\begin{array}{l}\mathrm{AP}, \\
\mathrm{SCU}^{(2)}\end{array}$ & $\begin{array}{l}\mathrm{Q}, \mathrm{N} \\
\mathrm{M}\end{array}$ & $\begin{array}{l}38 . \\
4\end{array}$ & 0.2 & 0.5 & 2.5 & $-\overline{1}$ & Passa \\
\hline Fundação & $20 \times 20$ & $\begin{array}{l}\text { Elemento de } \\
\text { Fundação }\end{array}$ & $\begin{array}{l}\text { N.P. ( } \\
1)\end{array}$ & $\begin{array}{l}\text { N.P. ( } \\
1)\end{array}$ & 1.9 & 6.1 & 6.1 & $\begin{array}{l}\mathrm{AP}_{1} \\
\mathrm{SCU}^{(2)}\end{array}$ & $\begin{array}{l}\mathrm{Q}, \mathrm{N}, \\
\mathrm{M}\end{array}$ & $\begin{array}{l}38 . \\
4\end{array}$ & 0.2 & 0.5 & 2.5 & - & Passa \\
\hline
\end{tabular}




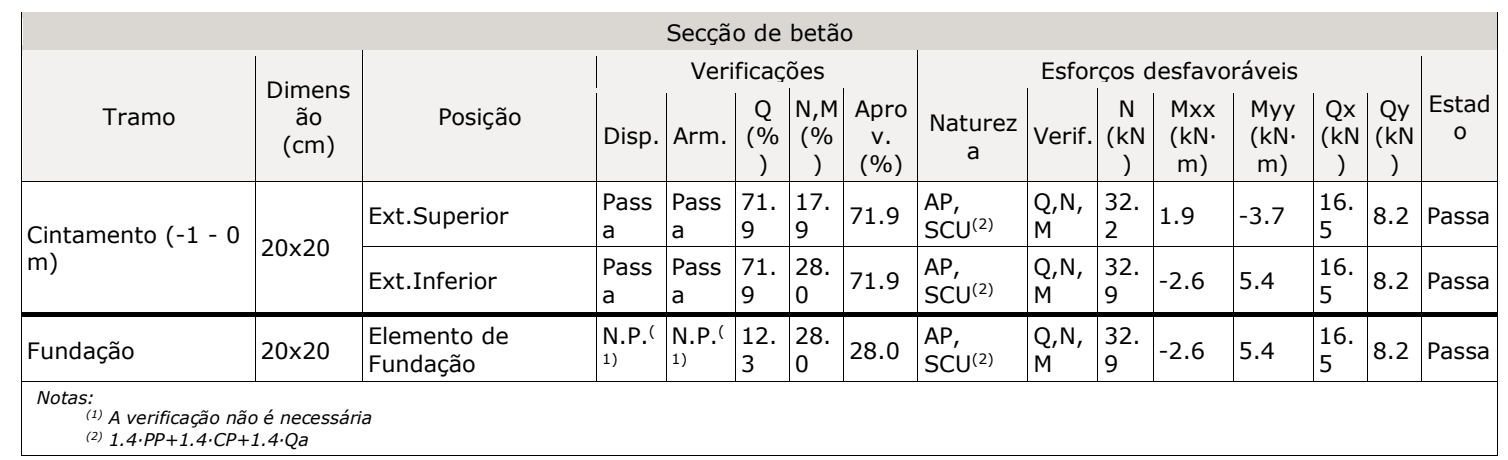

P5

\begin{tabular}{|c|c|c|c|c|c|c|c|c|c|c|c|c|c|c|c|}
\hline \multicolumn{16}{|c|}{ Secção de betão } \\
\hline \multirow[b]{2}{*}{ Tramo } & \multirow[b]{2}{*}{$\begin{array}{c}\text { Dimens } \\
\text { ão } \\
(\mathrm{cm})\end{array}$} & \multirow[b]{2}{*}{ Posição } & \multicolumn{5}{|c|}{ Verificações } & \multicolumn{7}{|c|}{ Esforços desfavoráveis } & \multirow[b]{2}{*}{$\begin{array}{c}\text { Estad } \\
0\end{array}$} \\
\hline & & & Disp. & Arm. & $\begin{array}{c}\mathrm{Q} \\
(\% \\
)\end{array}$ & $\begin{array}{c}\mathrm{N} \\
\mathrm{M} \\
(\% \\
)\end{array}$ & $\begin{array}{c}\text { Apro } \\
\text { V. } \\
(\%)\end{array}$ & $\begin{array}{c}\text { Naturez } \\
a\end{array}$ & $\begin{array}{c}\text { Verif } \\
.\end{array}$ & $\stackrel{\mathrm{N}}{(\mathrm{kN})}$ & $\begin{array}{c}M x x \\
(k N \cdot \\
m)\end{array}$ & $\begin{array}{l}\text { Myy } \\
(\mathrm{kN} \text {. } \\
\mathrm{m})\end{array}$ & $\begin{array}{c}\mathrm{Qx} \\
(\mathrm{kN})\end{array}$ & $\begin{array}{c}\text { Qy } \\
(\mathrm{kN} \\
)^{2}\end{array}$ & \\
\hline \multirow{2}{*}{$\begin{array}{l}\text { Cobertura }(0-3.15 \\
\mathrm{m})\end{array}$} & \multirow{2}{*}{$55 \times 15$} & Ext.Superior & $\begin{array}{l}\text { Pass } \\
\text { a }\end{array}$ & $\begin{array}{l}\text { Pass } \\
\text { a }\end{array}$ & $\begin{array}{l}36 . \\
4\end{array}$ & $\begin{array}{l}43 . \\
7\end{array}$ & 43.7 & $\begin{array}{l}\text { AP, } \\
\text { SCU }^{(2)}\end{array}$ & $\begin{array}{l}\mathrm{Q}, \mathrm{N}, \\
\mathrm{M}\end{array}$ & 88.2 & -0.4 & 30.4 & - & $-\overline{0}$ & Passa \\
\hline & & Ext.Inferior & \begin{tabular}{|l} 
Pass \\
a
\end{tabular} & $\begin{array}{l}\text { Pass } \\
\text { a }\end{array}$ & $\begin{array}{l}32 . \\
5\end{array}$ & $\begin{array}{l}15 . \\
8\end{array}$ & 32.5 & $\begin{array}{l}\text { AP, } \\
\text { SCU (2) }\end{array}$ & $\begin{array}{l}\mathrm{Q}, \mathrm{N}, \\
\mathrm{M}\end{array}$ & 95.9 & 0.2 & -7.8 & \begin{tabular}{|l|}
14.2 \\
\end{tabular} & $-\overline{0}$ & Passa \\
\hline \multirow{4}{*}{$\begin{array}{l}\text { Cintamento }(-1-0 \\
\mathrm{m})\end{array}$} & \multirow{4}{*}{$55 \times 15$} & \multirow[b]{2}{*}{ Ext.Superior } & \multirow{2}{*}{$\begin{array}{l}\text { Pass } \\
\text { a }\end{array}$} & \multirow{2}{*}{$\begin{array}{l}\text { Pass } \\
\text { a }\end{array}$} & \multirow{2}{*}{$\begin{array}{l}87 . \\
0\end{array}$} & \multirow{2}{*}{$\begin{array}{l}16 . \\
8\end{array}$} & \multirow{2}{*}{87.0} & $\mathrm{AP}^{(3)}$ & Q & $\begin{array}{l}130 . \\
6\end{array}$ & 1.2 & 12.6 & \begin{tabular}{|l|}
- \\
33.8 \\
\end{tabular} & 3.8 & \multirow[b]{2}{*}{ Passa } \\
\hline & & & & & & & & $\begin{array}{l}\text { AP, } \\
S C U^{(2)}\end{array}$ & $\mathrm{N}, \mathrm{M}$ & $\begin{array}{l}154 . \\
8\end{array}$ & 1.3 & 12.3 & \begin{tabular}{|l|}
- \\
32.2
\end{tabular} & 4.0 & \\
\hline & & \multirow{2}{*}{ Ext.Inferior } & \multirow{2}{*}{$\begin{array}{l}\text { Pass } \\
\text { a }\end{array}$} & \multirow{2}{*}{$\begin{array}{l}\text { Pass } \\
\text { a }\end{array}$} & \multirow{2}{*}{$\begin{array}{l}43 . \\
7\end{array}$} & \multirow{2}{*}{$\begin{array}{l}13 . \\
5\end{array}$} & \multirow{2}{*}{43.7} & $\mathrm{AP}^{(3)}$ & Q & $\begin{array}{l}132 . \\
2\end{array}$ & -0.8 & -5.9 & \begin{tabular}{|l|}
33.8 \\
\end{tabular} & 3.8 & \multirow[b]{2}{*}{ Passa } \\
\hline & & & & & & & & $\begin{array}{l}\text { AP, } \\
\mathrm{SCU}^{(2)}\end{array}$ & $\mathrm{N}, \mathrm{M}$ & $\begin{array}{l}156 . \\
3\end{array}$ & -0.9 & -5.4 & \begin{tabular}{|l|} 
\\
32.2
\end{tabular} & 4.0 & \\
\hline \multirow{2}{*}{ Fundação } & \multirow{2}{*}{$55 \times 15$} & \multirow{2}{*}{$\begin{array}{l}\text { Elemento de } \\
\text { Fundação }\end{array}$} & \multirow{2}{*}{$\begin{array}{l}\text { N.P. } \\
\text { 1) }\end{array}$} & \multirow{2}{*}{$\begin{array}{l}\text { N.P. } \\
1)\end{array}$} & \multirow{2}{*}{$\begin{array}{l}14 . \\
9\end{array}$} & \multirow{2}{*}{$\begin{array}{l}13 . \\
5\end{array}$} & \multirow{2}{*}{14.9} & $\mathrm{AP}^{(3)}$ & Q & $\begin{array}{l}132 . \\
2\end{array}$ & -0.8 & -5.9 & \begin{tabular}{|l|}
33.8 \\
\end{tabular} & 3.8 & \multirow[b]{2}{*}{ Passa } \\
\hline & & & & & & & & $\begin{array}{l}\text { AP, } \\
\mathrm{SCU}^{(2)}\end{array}$ & $\mathrm{N}, \mathrm{M}$ & 156. & -0.9 & -5.4 & $\begin{array}{l}- \\
32.2\end{array}$ & 4.0 & \\
\hline $\begin{array}{l}\text { Notas: } \\
\text { (1) } A \text { verificação não } \\
\text { (2) } 1.4 \cdot P P+1.4 \cdot C P+1 \\
\text { (3) } 1.4 \cdot P P+1.4 \cdot C P\end{array}$ & $\begin{array}{l}\text { Pé necessá } \\
1.4 \cdot \mathrm{Oa}\end{array}$ & & & & & & & & & & & & & & \\
\hline
\end{tabular}

P6

\begin{tabular}{|c|c|c|c|c|c|c|c|c|c|c|c|c|c|c|c|}
\hline \multicolumn{16}{|c|}{ Secção de betão } \\
\hline \multirow[b]{2}{*}{ Tramo } & \multirow[b]{2}{*}{$\begin{array}{c}\text { Dimens } \\
\text { ão } \\
(\mathrm{cm})\end{array}$} & \multirow[b]{2}{*}{ Posição } & \multicolumn{5}{|c|}{ Verificações } & \multicolumn{7}{|c|}{ Esforços desfavoráveis } & \multirow[b]{2}{*}{$\begin{array}{c}\text { Estad } \\
0\end{array}$} \\
\hline & & & Disp. & Arm. & $\begin{array}{c}Q \\
(\% \\
)\end{array}$ & \begin{tabular}{|c|}
$\mathrm{N}$ \\
$\mathrm{M}$ \\
$(\%$ \\
)
\end{tabular} & $\begin{array}{c}\text { Apro } \\
\text { v. } \\
(\%)\end{array}$ & $\begin{array}{c}\text { Naturez } \\
\text { a }\end{array}$ & $\begin{array}{c}\text { Verif } \\
\cdot\end{array}$ & $\stackrel{\mathrm{N}}{(\mathrm{kN})}$ & $\begin{array}{l}\text { Mxx } \\
(\mathrm{kN} . \\
\mathrm{m})\end{array}$ & $\begin{array}{c}\text { Myy } \\
(\mathrm{kN} \text {. } \\
\mathrm{m})\end{array}$ & $\begin{array}{c}\text { Qx } \\
(\mathrm{kN} \\
)\end{array}$ & $\begin{array}{c}\text { Qy } \\
(\mathrm{kN} \\
)\end{array}$ & \\
\hline \multirow{2}{*}{$\begin{array}{l}\text { Cobertura }(0-3.15 \\
\text { m) }\end{array}$} & \multirow{2}{*}{$20 \times 20$} & Ext.Superior & \begin{tabular}{|l|} 
Pass \\
a
\end{tabular} & $\begin{array}{l}\text { Pass } \\
\text { a }\end{array}$ & $\begin{array}{l}14 . \\
9\end{array}$ & $\begin{array}{l}25 . \\
0\end{array}$ & 25.0 & $\begin{array}{l}\mathrm{AP} \\
\mathrm{SCU}\end{array}$ & $\begin{array}{l}Q, N, \\
M\end{array}$ & 51.1 & 0.3 & -4.7 & 3.8 & 0.3 & Passa \\
\hline & & Ext.Inferior & $\begin{array}{l}\text { Pass } \\
\text { a } \\
\end{array}$ & $\begin{array}{l}\text { Pass } \\
\mathrm{a} \\
\end{array}$ & $\begin{array}{l}14 . \\
9\end{array}$ & $\begin{array}{l}29 . \\
3\end{array}$ & 29.3 & $\begin{array}{l}\mathrm{AP} \\
\mathrm{SCU}(2)\end{array}$ & $\begin{array}{l}Q, N, \\
M\end{array}$ & 54.8 & -0.5 & 5.6 & 3.8 & 0.3 & Passa \\
\hline $\begin{array}{l}\text { Cintamento }(-1-0 \\
\mathrm{m})\end{array}$ & $20 \times 20$ & Ext.Superior & $\left|\begin{array}{l}\text { Pass } \\
\text { a }\end{array}\right|$ & $\begin{array}{l}\text { Pass } \\
\text { a }\end{array}$ & $\begin{array}{l}68 . \\
3\end{array}$ & $\begin{array}{l}25 \\
4\end{array}$ & 68.3 & $\mathrm{AP}^{(3)}$ & Q & 85.1 & -2.3 & -5.5 & $\begin{array}{l}16 . \\
6\end{array}$ & - & Passa \\
\hline
\end{tabular}




\section{Pontifícla Universidade Católlica}

DO RIO DE JANEIRO

\begin{tabular}{|c|c|c|c|c|c|c|c|c|c|c|c|c|c|c|c|}
\hline \multicolumn{16}{|c|}{ Secção de betão } \\
\hline \multirow[b]{2}{*}{ Tramo } & \multirow[b]{2}{*}{$\begin{array}{c}\text { Dimens } \\
\text { ão } \\
(\mathrm{cm})\end{array}$} & \multirow[b]{2}{*}{ Posição } & \multicolumn{5}{|c|}{ Verificações } & \multicolumn{7}{|c|}{ Esforços desfavoráveis } & \multirow{3}{*}{$\begin{array}{c}\text { Estad } \\
0\end{array}$} \\
\hline & & & \multirow[t]{2}{*}{ Disp. } & \multirow[t]{2}{*}{ Arm. } & \multirow[t]{2}{*}{$\begin{array}{c}\mathrm{Q} \\
(\% \\
)\end{array}$} & \multirow[t]{2}{*}{\begin{tabular}{|c|}
$\mathrm{N}$ \\
$\mathrm{M}$ \\
$(\%$ \\
)
\end{tabular}} & \multirow[t]{2}{*}{$\begin{array}{c}\text { Apro } \\
\text { V. } \\
(\%) \\
\end{array}$} & \multirow{2}{*}{\begin{tabular}{|l}
$\begin{array}{c}\text { Naturez } \\
\text { a }\end{array}$ \\
$\begin{array}{l}\text { AP, } \\
\mathrm{SCU}^{(2)}\end{array}$
\end{tabular}} & \multirow{2}{*}{\begin{tabular}{|c|}
$\begin{array}{c}\text { Verif } \\
\cdot\end{array}$ \\
$N, M$
\end{tabular}} & \multirow{2}{*}{\begin{tabular}{|l|}
$\begin{array}{l}N \\
(\mathrm{kN})\end{array}$ \\
102. \\
6
\end{tabular}} & \multirow{2}{*}{\begin{tabular}{|c}
$\left.\begin{array}{c}M \times x \\
(k N \\
m\end{array}\right)$ \\
-2.3
\end{tabular}} & \multirow{2}{*}{\begin{tabular}{|c} 
Myy \\
$(\mathrm{kN}$. \\
$\mathrm{m})$
\end{tabular}} & \multirow{2}{*}{\begin{tabular}{|c|}
$\begin{array}{c}\text { Qx } \\
(\mathrm{kN} \\
)\end{array}$ \\
15. \\
8
\end{tabular}} & $\begin{array}{c}\text { Qy } \\
(\mathrm{kN} \\
)\end{array}$ & \\
\hline & & & & & & & & & & & & & & - & \\
\hline & & \multirow{2}{*}{ Ext.Inferior } & \multirow{2}{*}{$\begin{array}{l}\text { Pass } \\
\text { a }\end{array}$} & \multirow{2}{*}{$\begin{array}{l}\text { Pass } \\
\text { a }\end{array}$} & \multirow{2}{*}{$\begin{array}{l}66 . \\
4\end{array}$} & \multirow{2}{*}{$\begin{array}{l}19 . \\
8\end{array}$} & \multirow{2}{*}{66.4} & $A P^{(3)}$ & Q & 85.9 & 1.5 & 3.7 & $\begin{array}{l}16 . \\
6\end{array}$ & $-\overline{6}$ & \multirow{2}{*}{ Passa } \\
\hline & & & & & & & & $\begin{array}{l}\mathrm{AP}_{1} \\
\mathrm{SCU}\end{array}$ & $\mathrm{N}, \mathrm{M}$ & $\begin{array}{l}103 . \\
3\end{array}$ & 1.6 & 3.5 & $\begin{array}{l}15 . \\
8\end{array}$ & $\begin{array}{l} \\
7.1\end{array}$ & \\
\hline \multirow{2}{*}{ Fundação } & \multirow{2}{*}{$20 \times 20$} & \multirow{2}{*}{$\begin{array}{l}\text { Elemento de } \\
\text { Fundação }\end{array}$} & \multirow{2}{*}{$\begin{array}{l}\text { N.P. } \\
1)\end{array}$} & \multirow{2}{*}{$\begin{array}{l}\text { N.P. } \\
1)\end{array}$} & \multirow{2}{*}{$\begin{array}{l}12 . \\
0\end{array}$} & \multirow{2}{*}{$\begin{array}{l}19 . \\
8\end{array}$} & \multirow{2}{*}{19.8} & $A P^{(3)}$ & $\mathrm{Q}$ & 85.9 & 1.5 & 3.7 & $\begin{array}{l}16 . \\
6\end{array}$ & $-\overline{6}$ & \multirow{2}{*}{ Passa } \\
\hline & & & & & & & & $\begin{array}{l}\mathrm{AP}, \\
\mathrm{SCU}(2)\end{array}$ & $\mathrm{N}, \mathrm{M}$ & $\begin{array}{l}103 . \\
3\end{array}$ & 1.6 & 3.5 & $\begin{array}{l}15 . \\
8\end{array}$ & -7 & \\
\hline $\begin{array}{l}\text { Notas: } \\
\text { (1) A verifica } \\
\text { (2) } 1.4 \cdot P P+1 \\
\text { (3) } 1.4 \cdot P P+1\end{array}$ & $\begin{array}{l}\text { necessári } \\
4 \cdot Q a\end{array}$ & & & & & & & & & & & & & & \\
\hline
\end{tabular}

P7

\begin{tabular}{|c|c|c|c|c|c|c|c|c|c|c|c|c|c|c|c|}
\hline \multicolumn{16}{|c|}{ Secção de betão } \\
\hline \multirow[b]{2}{*}{ Tramo } & \multirow[b]{2}{*}{$\begin{array}{c}\text { Dimens } \\
\text { ão } \\
(\mathrm{cm})\end{array}$} & \multirow[b]{2}{*}{ Posição } & \multicolumn{5}{|c|}{ Verificações } & \multicolumn{7}{|c|}{ Esforços desfavoráveis } & \multirow[b]{2}{*}{$\begin{array}{c}\text { Estad } \\
0\end{array}$} \\
\hline & & & Disp. & Arm. & $\begin{array}{c}\mathrm{Q} \\
(\% \\
)\end{array}$ & \begin{tabular}{|c|}
$\mathrm{N}$ \\
$\mathrm{M}$ \\
$(\%$ \\
)
\end{tabular} & $\begin{array}{c}\text { Apro } \\
\text { V. } \\
(\%)\end{array}$ & $\begin{array}{c}\text { Naturez } \\
a\end{array}$ & $\begin{array}{c}\text { Verif } \\
.\end{array}$ & $\begin{array}{c}\mathrm{N} \\
(\mathrm{kN})\end{array}$ & $\begin{array}{l}\mathrm{Mxx} \\
(\mathrm{kN} \cdot \\
\mathrm{m})\end{array}$ & $\begin{array}{l}\text { Myy } \\
(\mathrm{kN} \text {. } \\
\mathrm{m})\end{array}$ & $\begin{array}{c}\mathrm{Qx} \\
(\mathrm{kN})\end{array}$ & $\begin{array}{c}\text { Qy } \\
(\mathrm{kN} \\
)\end{array}$ & \\
\hline \multirow{2}{*}{$\begin{array}{l}\text { Cobertura }(0-3.15 \\
\mathrm{m})\end{array}$} & \multirow{2}{*}{$20 \times 20$} & Ext.Superior & \begin{tabular}{|l} 
Pass \\
a
\end{tabular} & $\begin{array}{l}\text { Pass } \\
\text { a }\end{array}$ & 7.2 & $\begin{array}{l}17 . \\
7\end{array}$ & 17.7 & $\begin{array}{l}\text { AP, } \\
S C U^{(2)}\end{array}$ & $\begin{array}{l}Q, N, \\
M\end{array}$ & 48.8 & -0.1 & 3.0 & -1.9 & $-\overline{0}$ & Passa \\
\hline & & Ext.Inferior & \begin{tabular}{|l} 
Pass \\
$\mathrm{a}$
\end{tabular} & $\begin{array}{l}\text { Pass } \\
a\end{array}$ & 7.2 & $\begin{array}{l}15 . \\
2\end{array}$ & 15.2 & $\begin{array}{l}\mathrm{AP}, \\
\mathrm{SCU} \mathrm{U}^{(2)}\end{array}$ & $\begin{array}{l}Q, N, \\
M\end{array}$ & 52.5 & 0.1 & -2.0 & -1.9 & $-\overline{0}$ & Passa \\
\hline \multirow{4}{*}{$\begin{array}{l}\text { Cintamento }(-1-0 \\
\mathrm{m})\end{array}$} & \multirow{4}{*}{$20 \times 20$} & \multirow{2}{*}{ Ext.Superior } & \multirow{2}{*}{$\begin{array}{l}\text { Pass } \\
\text { a }\end{array}$} & \multirow{2}{*}{$\begin{array}{l}\text { Pass } \\
\text { a }\end{array}$} & \multirow{2}{*}{$\begin{array}{l}93 . \\
6\end{array}$} & \multirow{2}{*}{$\begin{array}{l}33 . \\
5\end{array}$} & \multirow{2}{*}{93.6} & $\mathrm{AP}^{(3)}$ & Q & 98.5 & 3.5 & 6.9 & \begin{tabular}{|l|}
21.7 \\
\end{tabular} & $\begin{array}{l}10 . \\
3\end{array}$ & \multirow{2}{*}{ Passa } \\
\hline & & & & & & & & $\begin{array}{l}\text { AP, } \\
\text { SCU(2) }\end{array}$ & $\mathrm{N}, \mathrm{M}$ & $\begin{array}{l}116 . \\
0\end{array}$ & 3.6 & 6.8 & $\begin{array}{l} \\
21.4\end{array}$ & $\begin{array}{l}10 . \\
7\end{array}$ & \\
\hline & & \multirow{2}{*}{ Ext.Inferior } & \multirow{2}{*}{$\begin{array}{l}\text { Pass } \\
\text { a }\end{array}$} & \multirow{2}{*}{$\begin{array}{l}\text { Pass } \\
\text { a }\end{array}$} & \multirow{2}{*}{$\begin{array}{l}88 . \\
6\end{array}$} & \multirow{2}{*}{$\begin{array}{l}25 . \\
6\end{array}$} & \multirow{2}{*}{88.6} & $\mathrm{AP}^{(3)}$ & Q & 99.3 & -2.2 & -5.0 & \begin{tabular}{|l|} 
\\
21.7
\end{tabular} & $\begin{array}{l}10 . \\
3 \\
\end{array}$ & \multirow{2}{*}{ Passa } \\
\hline & & & & & & & & $\begin{array}{l}\mathrm{AP}, \\
\mathrm{SCU}\end{array}$ & $\mathrm{N}, \mathrm{M}$ & $\begin{array}{l}116 . \\
7\end{array}$ & -2.3 & -4.9 & \begin{tabular}{|l|}
- \\
21.4 \\
\end{tabular} & $\begin{array}{l}10 . \\
7\end{array}$ & \\
\hline \multirow{2}{*}{ Fundação } & \multirow{2}{*}{$20 \times 20$} & \multirow{2}{*}{$\begin{array}{l}\text { Elemento de } \\
\text { Fundação }\end{array}$} & \multirow{2}{*}{$\begin{array}{l}\text { N.P. } \\
\text { 1) }\end{array}$} & \multirow{2}{*}{$\begin{array}{l}\text { N.P. } \\
1)\end{array}$} & \multirow{2}{*}{$\begin{array}{l}16 . \\
0\end{array}$} & \multirow{2}{*}{$\begin{array}{l}25 . \\
6\end{array}$} & \multirow{2}{*}{25.6} & $A P^{(3)}$ & Q & 99.3 & -2.2 & -5.0 & \begin{tabular}{|l|} 
\\
21.7
\end{tabular} & $\begin{array}{l}10 . \\
3\end{array}$ & \multirow{2}{*}{ Passa } \\
\hline & & & & & & & & $\begin{array}{l}\mathrm{AP}_{1} \\
\mathrm{SCU}^{(2)}\end{array}$ & $\mathrm{N}, \mathrm{M}$ & $\begin{array}{l}116 . \\
7\end{array}$ & -2.3 & -4.9 & \begin{tabular}{|l|}
21.4 \\
\end{tabular} & $\begin{array}{l}10 . \\
7\end{array}$ & \\
\hline $\begin{array}{l}\text { Notas: } \\
\text { (1) } A \text { verificação nã } \\
\text { (2) } 1.4 \cdot P P+1.4 \cdot C P+ \\
\text { (3) } 1.4 \cdot P P+1.4 \cdot C P\end{array}$ & $\begin{array}{l}\text { oé necessá } \\
1.4 . \mathrm{Oa}\end{array}$ & & & & & & & & & & & & & & \\
\hline
\end{tabular}

P8

\begin{tabular}{|c|c|c|c|c|c|c|c|c|c|c|c|c|c|c|c|}
\hline \multicolumn{16}{|c|}{ Secção de betão } \\
\hline \multirow[b]{2}{*}{ Tramo } & \multirow[b]{2}{*}{$\begin{array}{c}\text { Dimens } \\
\text { ão } \\
(\mathrm{cm})\end{array}$} & \multirow[b]{2}{*}{ Posição } & \multicolumn{5}{|c|}{ Verificações } & \multicolumn{7}{|c|}{ Esforços desfavoráveis } & \multirow[b]{2}{*}{$\begin{array}{c}\text { Estad } \\
0\end{array}$} \\
\hline & & & Disp. & Arm. & $\begin{array}{c}\mathrm{Q} \\
(\% \\
) \\
\end{array}$ & $\begin{array}{c}\mathrm{N}, \mathrm{M} \\
(\% \\
)\end{array}$ & $\begin{array}{c}\text { Apro } \\
\text { V. } \\
(\%) \\
\end{array}$ & $\begin{array}{c}\text { Naturez } \\
\text { a }\end{array}$ & $\begin{array}{c}\text { Verif } \\
.\end{array}$ & $\begin{array}{c}\mathrm{N} \\
(\mathrm{kN} \\
)\end{array}$ & $\begin{array}{c}M \times x \\
(k N . \\
m)\end{array}$ & $\begin{array}{c}\text { Myy } \\
(\mathrm{kN} . \\
\mathrm{m})\end{array}$ & $\begin{array}{c}\text { Qx } \\
(\mathrm{kN} \\
) \\
\end{array}$ & $\begin{array}{c}\text { Qy } \\
(\mathrm{kN} \\
) \\
\end{array}$ & \\
\hline \multirow{2}{*}{$\begin{array}{l}\text { Cobertura }(0-3.15 \\
\mathrm{m})\end{array}$} & \multirow{2}{*}{$20 \times 20$} & Ext.Superior & \begin{tabular}{|l|} 
Pass \\
$\mathrm{a}$
\end{tabular} & $\begin{array}{l}\text { Pass } \\
\text { a }\end{array}$ & \begin{tabular}{|l|}
14. \\
8
\end{tabular} & \begin{tabular}{|l|}
24. \\
5
\end{tabular} & 24.5 & $\begin{array}{l}\text { AP, } \\
S^{\prime} U^{(2)}\end{array}$ & $\begin{array}{l}\mathrm{Q}, \mathrm{N}, \\
\mathrm{M}\end{array}$ & \begin{tabular}{|l|}
31. \\
0
\end{tabular} & -0.4 & -4.6 & 3.8 & \begin{tabular}{|l|}
- \\
0.3 \\
\end{tabular} & Passa \\
\hline & & Ext.Inferior & \begin{tabular}{|l|} 
Pass \\
$\mathrm{a}$
\end{tabular} & \begin{tabular}{|l} 
Pass \\
a
\end{tabular} & $\begin{array}{l}14 . \\
8\end{array}$ & $\begin{array}{l}29 . \\
8\end{array}$ & 29.8 & $\begin{array}{l}\mathrm{AP}^{\prime} \\
\mathrm{SCU}\end{array}$ & $\begin{array}{l}\mathrm{Q}, \mathrm{N}, \\
\mathrm{M}\end{array}$ & $\begin{array}{l}34 . \\
7\end{array}$ & 0.5 & 5.6 & 3.8 & $\begin{array}{l} \\
0.3 \\
\end{array}$ & Passa \\
\hline \multirow{2}{*}{$\begin{array}{l}\text { Cintamento }(-1-0 \\
\mathrm{m})\end{array}$} & \multirow{2}{*}{$20 \times 20$} & Ext.Superior & \begin{tabular}{|l|} 
Pass \\
a
\end{tabular} & $\begin{array}{l}\text { Pass } \\
\text { a }\end{array}$ & $\begin{array}{l}92 . \\
2\end{array}$ & $\begin{array}{l}30 . \\
3\end{array}$ & 92.2 & $\mathrm{AP}^{(3)}$ & $\begin{array}{l}\mathrm{Q}, \mathrm{N}, \\
\mathrm{M}\end{array}$ & $\begin{array}{l}71 . \\
9\end{array}$ & -1.4 & -7.7 & $\begin{array}{l}23 . \\
5\end{array}$ & $\begin{array}{l} \\
4.4\end{array}$ & Passa \\
\hline & & Ext.Inferior & \begin{tabular}{|l|} 
Pass \\
a
\end{tabular} & $\begin{array}{l}\text { Pass } \\
\text { a }\end{array}$ & $\begin{array}{l}92 . \\
1\end{array}$ & $\begin{array}{l}21 . \\
8\end{array}$ & 92.1 & $\mathrm{AP}^{(3)}$ & Q & $\begin{array}{l}72 . \\
7\end{array}$ & 1.1 & 5.2 & $\begin{array}{l}23 . \\
5\end{array}$ & $-\overline{4}$ & Passa \\
\hline
\end{tabular}




\section{Pontifícia Universidade Católlca}

DO RIO DE JANEIRO

\begin{tabular}{|c|c|c|c|c|c|c|c|c|c|c|c|c|c|c|c|}
\hline \multicolumn{16}{|c|}{ Secção de betão } \\
\hline \multirow{3}{*}{ Tramo } & \multirow{3}{*}{$\begin{array}{l}\text { Dimens } \\
\text { ão } \\
(\mathrm{cm})\end{array}$} & \multirow{3}{*}{ Posição } & \multicolumn{5}{|c|}{ Verificações } & \multicolumn{7}{|c|}{ Esforços desfavoráveis } & \multirow{3}{*}{$\begin{array}{c}\text { Estad } \\
0\end{array}$} \\
\hline & & & Disp. & Arm. & $\begin{array}{c}\mathrm{Q} \\
(\% \\
)\end{array}$ & $\begin{array}{c}\mathrm{N}, \mathrm{M} \\
(\% \\
)\end{array}$ & $\begin{array}{c}\text { Apro } \\
\text { V. } \\
(\%)\end{array}$ & $\begin{array}{c}\text { Naturez } \\
\text { a }\end{array}$ & $\begin{array}{c}\text { Verif } \\
\cdot\end{array}$ & $\begin{array}{c}\mathrm{N} \\
(\mathrm{kN} \\
)\end{array}$ & $\begin{array}{c}\mathrm{Mxx} \\
(\mathrm{kN} \cdot \\
\mathrm{m})\end{array}$ & $\begin{array}{c}\text { Myy } \\
(\mathrm{kN} . \\
\mathrm{m})\end{array}$ & $\begin{array}{c}\text { Qx } \\
(\mathrm{kN} \\
)\end{array}$ & $\begin{array}{c}\text { Qy } \\
(\mathrm{kN} \\
)^{2} \\
\end{array}$ & \\
\hline & & & & & & & & $\begin{array}{l}\mathrm{AP}, \\
\mathrm{SCU}^{(2)}\end{array}$ & $N, M$ & $\begin{array}{l}86 . \\
6 \\
\end{array}$ & 1.1 & 5.1 & $\begin{array}{l}22 . \\
8\end{array}$ & - & \\
\hline \multirow{2}{*}{ Fundação } & \multirow{2}{*}{$20 \times 20$} & \multirow{2}{*}{$\begin{array}{l}\text { Elemento de } \\
\text { Fundação }\end{array}$} & \multirow{2}{*}{$\begin{array}{l}\text { N.P. } \\
1)\end{array}$} & \multirow{2}{*}{$\begin{array}{l}\text { N.P. } \\
1)\end{array}$} & \multirow{2}{*}{$\begin{array}{l}15 . \\
9\end{array}$} & \multirow{2}{*}{$\begin{array}{l}21 . \\
8\end{array}$} & \multirow{2}{*}{21.8} & $\mathrm{AP}^{(3)}$ & Q & $\begin{array}{l}72 . \\
7\end{array}$ & 1.1 & 5.2 & $\begin{array}{l}23 . \\
5\end{array}$ & - & \multirow{2}{*}{ Passa } \\
\hline & & & & & & & & $\begin{array}{l}\text { AP, } \\
S C U^{(2)}\end{array}$ & $N, M$ & $\begin{array}{l}86 . \\
6 \\
\end{array}$ & 1.1 & 5.1 & $\begin{array}{l}22 . \\
8\end{array}$ & -7 & \\
\hline \multicolumn{16}{|c|}{$\begin{array}{l}\text { Notas: } \\
\text { (1) } A \text { verificação não é necessária } \\
\text { (2) } 1.4 \cdot P P+1.4 \cdot C P+1.4 \cdot Q a \\
\text { (3) } 1.4 \cdot P P+1.4 \cdot C P\end{array}$} \\
\hline
\end{tabular}

P9

\begin{tabular}{|c|c|c|c|c|c|c|c|c|c|c|c|c|c|c|c|}
\hline \multicolumn{16}{|c|}{ Secção de betão } \\
\hline \multirow[b]{2}{*}{ Tramo } & \multirow[b]{2}{*}{$\begin{array}{c}\text { Dimens } \\
\text { ão } \\
(\mathrm{cm})\end{array}$} & \multirow[b]{2}{*}{ Posição } & \multicolumn{5}{|c|}{ Verificações } & \multicolumn{7}{|c|}{ Esforços desfavoráveis } & \multirow[b]{2}{*}{$\begin{array}{c}\text { Estad } \\
0\end{array}$} \\
\hline & & & Disp. & Arm. & $\begin{array}{c}\mathrm{Q} \\
(\% \\
)\end{array}$ & $\begin{array}{c}\mathrm{N}, \mathrm{M} \\
(\% \\
)\end{array}$ & \begin{tabular}{|c|} 
Apro \\
V. \\
$(\%)$
\end{tabular} & $\begin{array}{c}\text { Naturez } \\
a\end{array}$ & Verif. & $\begin{array}{c}\mathrm{N} \\
(\mathrm{kN} \\
)\end{array}$ & $\begin{array}{c}M \times x \\
(k N . \\
m)\end{array}$ & $\begin{array}{c}\text { Myy } \\
(\mathrm{kN} . \\
\mathrm{m})\end{array}$ & $\begin{array}{c}\text { Qx } \\
(\mathrm{kN} \\
)\end{array}$ & $\begin{array}{c}\text { Qy } \\
(\mathrm{kN} \\
)\end{array}$ & \\
\hline \multirow{2}{*}{$\begin{array}{l}\text { Cintamento (-1 - } 0 \\
\mathrm{~m})\end{array}$} & \multirow{2}{*}{$20 \times 20$} & Ext.Superior & \begin{tabular}{|l|} 
Pass \\
a
\end{tabular} & \begin{tabular}{|l|} 
Pass \\
$\mathrm{a}$
\end{tabular} & $\begin{array}{l}48 . \\
9\end{array}$ & $\begin{array}{l}11 . \\
4\end{array}$ & 48.9 & $\begin{array}{l}\mathrm{AP}, \\
\mathrm{SCU} \\
\end{array}$ & $\begin{array}{l}\mathrm{Q}, \mathrm{N}, \\
\mathrm{M}\end{array}$ & \begin{tabular}{|l|}
27. \\
3
\end{tabular} & 2.7 & -0.8 & 3.1 & $\begin{array}{l}12 . \\
2\end{array}$ & Passa \\
\hline & & Ext.Inferior & \begin{tabular}{|l|} 
Pass \\
a
\end{tabular} & \begin{tabular}{|l|} 
Pass \\
a
\end{tabular} & $\begin{array}{l}49 . \\
3\end{array}$ & $\begin{array}{l}17 . \\
1\end{array}$ & 49.3 & $\begin{array}{l}\mathrm{AP}, \\
\mathrm{SCU}\end{array}$ & $\begin{array}{l}\mathrm{Q}, \mathrm{N}, \\
\mathrm{M}\end{array}$ & \begin{tabular}{|l|}
28. \\
1
\end{tabular} & -4.0 & 0.9 & 3.1 & $\begin{array}{l}12 . \\
2\end{array}$ & Passa \\
\hline Fundação & $20 \times 20$ & $\begin{array}{l}\text { Elemento de } \\
\text { Fundação }\end{array}$ & $\begin{array}{l}\text { N.P.' } \\
1)\end{array}$ & $\begin{array}{l}\text { N.P. } \\
1)\end{array}$ & 8.4 & $\begin{array}{l}17 . \\
1\end{array}$ & 17.1 & $\begin{array}{l}\mathrm{AP}_{1} \\
\mathrm{SCU}\end{array}$ & $\begin{array}{l}, N, \\
M\end{array}$ & \begin{tabular}{|l|}
28. \\
1
\end{tabular} & -4.0 & 0.9 & 3.1 & $\begin{array}{l}12 . \\
2\end{array}$ & Passa \\
\hline $\begin{array}{l}\text { Notas: } \\
\text { (1) A verificação } n \\
\text { (2) } 1.4 \cdot P P+1.4 \cdot C P\end{array}$ & $\begin{array}{l}\text { necessá } \\
1 \cdot Q a\end{array}$ & & & & & & & & & & & & & & \\
\hline
\end{tabular}

P10

\begin{tabular}{|c|c|c|c|c|c|c|c|c|c|c|c|c|c|c|c|}
\hline \multicolumn{16}{|c|}{ Secção de betão } \\
\hline \multirow[b]{2}{*}{ Tramo } & \multirow[b]{2}{*}{$\begin{array}{c}\text { Dimensã } \\
0 \\
(\mathrm{~cm})\end{array}$} & \multirow[b]{2}{*}{ Posição } & \multicolumn{5}{|c|}{ Verificações } & \multicolumn{7}{|c|}{ Esforços desfavoráveis } & \multirow[b]{2}{*}{$\begin{array}{c}\text { Estad } \\
0\end{array}$} \\
\hline & & & Disp. & Arm. & $\begin{array}{c}Q \\
(\%)\end{array}$ & $\begin{array}{l}\mathrm{N}, \mathrm{M} \\
(\%)\end{array}$ & \begin{tabular}{|c|} 
Aprov \\
$\dot{(\%)}$
\end{tabular} & Natureza & Verif. & $\underset{(k N)}{N}$ & $\begin{array}{c}\mathrm{Mxx} \\
(\mathrm{kN} \cdot \mathrm{m} \\
)\end{array}$ & \begin{tabular}{|c|} 
Myy \\
$(\mathrm{kN} \cdot \mathrm{m}$ \\
)
\end{tabular} \mid & $\begin{array}{c}\mathrm{Qx} \\
(\mathrm{kN})\end{array}$ & $\begin{array}{c}\mathrm{Qy} \\
(\mathrm{kN})\end{array}$ & \\
\hline \multirow{2}{*}{$\begin{array}{l}\text { Cobertura (0 - } 3.15 \\
\text { m) }\end{array}$} & \multirow{2}{*}{$20 \times 20$} & Ext.Superior & \begin{tabular}{|l|} 
Pass \\
a
\end{tabular} & $\begin{array}{l}\text { Pass } \\
\text { a }\end{array}$ & 2.3 & $\begin{array}{l}12 . \\
4\end{array}$ & 12.4 & $\begin{array}{l}\mathrm{AP}, \\
\mathrm{SCU}(2)\end{array}$ & $\begin{array}{l}Q, N, \\
M\end{array}$ & 44.1 & -0.6 & 1.1 & -0.6 & -0.7 & Passa \\
\hline & & Ext.Inferior & \begin{tabular}{|l} 
Pass \\
a
\end{tabular} & $\begin{array}{l}\text { Pass } \\
\text { a }\end{array}$ & 2.3 & $\begin{array}{l}13 . \\
3\end{array}$ & 13.3 & $\begin{array}{l}\mathrm{AP}, \\
\mathrm{SCU}^{(2)}\end{array}$ & $\begin{array}{l}Q, N, \\
M\end{array}$ & 47.8 & 1.2 & -0.6 & -0.6 & -0.7 & Passa \\
\hline \multirow{3}{*}{$\begin{array}{l}\text { Cintamento (-1 - } 0 \\
\text { m) }\end{array}$} & \multirow{3}{*}{$20 \times 20$} & Ext.Superior & $\begin{array}{l}\text { Pass } \\
\text { a }\end{array}$ & $\begin{array}{l}\text { Pass } \\
\text { a }\end{array}$ & $\begin{array}{l}96 . \\
5\end{array}$ & $\begin{array}{l}33 . \\
7\end{array}$ & 96.5 & $\begin{array}{l}\mathrm{AP} \\
\mathrm{SCU}(2)\end{array}$ & $\begin{array}{l}, N, \\
M\end{array}$ & $\begin{array}{l}107 . \\
3\end{array}$ & -3.9 & 6.8 & \begin{tabular}{|l|}
21.4 \\
\end{tabular} & \begin{tabular}{|l|}
12.5 \\
\end{tabular} & Passa \\
\hline & & \multirow{2}{*}{ Ext.Inferior } & \multirow{2}{*}{$\begin{array}{l}\text { Pass } \\
\text { a }\end{array}$} & \multirow{2}{*}{$\begin{array}{l}\text { Pass } \\
\text { a }\end{array}$} & \multirow{2}{*}{$\begin{array}{l}92 . \\
7\end{array}$} & \multirow{2}{*}{$\begin{array}{l}26 . \\
6\end{array}$} & \multirow{2}{*}{92.7} & $\mathrm{AP}^{(3)}$ & Q & 93.1 & 2.9 & -4.9 & \begin{tabular}{|l|}
20.8 \\
\end{tabular} & \begin{tabular}{|l|}
12.2 \\
\end{tabular} & \multirow{2}{*}{ Passa } \\
\hline & & & & & & & & $\begin{array}{l}\text { AP, } \\
\text { SCU(2) }\end{array}$ & $\mathrm{N}, \mathrm{M}$ & $\begin{array}{l}108 \\
1\end{array}$ & 3.0 & -5.0 & $-\overline{21.4}$ & $\begin{array}{l} \\
12.5 \\
\end{array}$ & \\
\hline Fundação & $20 \times 20$ & $\begin{array}{l}\text { Elemento de } \\
\text { Fundação }\end{array}$ & $\begin{array}{l}\text { N.P. }(1 \\
)^{\prime}\end{array}$ & N.P. ${ }^{1}$ & $\begin{array}{l}16 . \\
5\end{array}$ & \begin{tabular}{l|l}
26. \\
6
\end{tabular} & 26.6 & $\begin{array}{l}\mathrm{AP} \\
\mathrm{SCU}(2)\end{array}$ & $\begin{array}{l}\mathrm{Q}, \mathrm{N}, \\
\mathrm{M}\end{array}$ & $\begin{array}{l}108 . \\
1\end{array}$ & 3.0 & -5.0 & \begin{tabular}{|l|}
21.4 \\
\end{tabular} & \begin{tabular}{|l|}
12.5 \\
\end{tabular} & Passa \\
\hline \multicolumn{16}{|c|}{$\begin{array}{l}\text { Notas: } \\
\text { (1) A verificação não é necessária } \\
\text { (2) } 1.4 \cdot P P+1.4 \cdot C P+1.4 \cdot Q a \\
\text { (3) } 1.4 \cdot P P+1.4 \cdot C P\end{array}$} \\
\hline
\end{tabular}




\section{P11}

\begin{tabular}{|c|c|c|c|c|c|c|c|c|c|c|c|c|c|c|c|}
\hline \multicolumn{16}{|c|}{ Secção de betão } \\
\hline \multirow[b]{2}{*}{ Tramo } & \multirow[b]{2}{*}{$\begin{array}{l}\text { Dimens } \\
\text { ão } \\
(\mathrm{cm})\end{array}$} & \multirow[b]{2}{*}{ Posição } & \multicolumn{5}{|c|}{ Verificações } & \multicolumn{7}{|c|}{ Esforços desfavoráveis } & \multirow[b]{2}{*}{$\begin{array}{c}\text { Estad } \\
0\end{array}$} \\
\hline & & & Disp. & Arm. & $\begin{array}{c}\mathrm{Q} \\
(\% \\
)\end{array}$ & $\begin{array}{c}\mathrm{N} \\
\mathrm{M} \\
(\% \\
)\end{array}$ & $\begin{array}{c}\text { Apro } \\
\text { V. } \\
(\%)\end{array}$ & $\begin{array}{c}\text { Naturez } \\
a\end{array}$ & Verif & $\stackrel{\mathrm{N}}{(\mathrm{kN})}$ & $\begin{array}{l}\mathrm{Mxx} \\
(\mathrm{kN} \\
\mathrm{m})\end{array}$ & $\begin{array}{l}\text { Myy } \\
(\mathrm{kN} \text {. } \\
\mathrm{m})\end{array}$ & $\begin{array}{c}Q x \\
(\mathrm{kN} \\
)\end{array}$ & $\begin{array}{c}\text { Qy } \\
(\mathrm{kN})\end{array}$ & \\
\hline \multirow{2}{*}{$\begin{array}{l}\text { Cobertura }(0-3.15 \\
\mathrm{m})\end{array}$} & \multirow{2}{*}{$20 \times 20$} & Ext.Superior & \begin{tabular}{|l} 
Pass \\
a
\end{tabular} & $\begin{array}{l}\text { Pass } \\
\text { a }\end{array}$ & 0.9 & $\begin{array}{l}36 . \\
2\end{array}$ & 36.2 & $\begin{array}{l}\mathrm{AP}_{1} \\
\mathrm{SCU}^{(2)}\end{array}$ & $\begin{array}{l}\mathrm{Q}, \mathrm{N}, \\
\mathrm{M}\end{array}$ & $\begin{array}{l}151 . \\
4\end{array}$ & -0.2 & 0.0 & 0.3 & -0.3 & Passa \\
\hline & & Ext.Inferior & \begin{tabular}{|l} 
Pass \\
a
\end{tabular} & $\begin{array}{l}\text { Pass } \\
a\end{array}$ & 0.9 & $\begin{array}{l}39 . \\
2\end{array}$ & 39.2 & $\begin{array}{l}\mathrm{AP} \\
\mathrm{SCU}^{(2)}\end{array}$ & $\begin{array}{l}\mathrm{Q}, \mathrm{N}, \\
\mathrm{M}\end{array}$ & $\begin{array}{l}155 . \\
1\end{array}$ & 0.6 & 1.0 & 0.3 & -0.3 & Passa \\
\hline \multirow{3}{*}{$\begin{array}{l}\text { Cintamento }(-1-0 \\
\mathrm{m})\end{array}$} & \multirow{3}{*}{$20 \times 20$} & \multirow{2}{*}{ Ext.Superior } & \multirow{2}{*}{$\begin{array}{l}\text { Pass } \\
\text { a }\end{array}$} & \multirow{2}{*}{$\begin{array}{l}\text { Pass } \\
\text { a }\end{array}$} & \multirow{2}{*}{$\begin{array}{l}32 . \\
1\end{array}$} & \multirow{2}{*}{$\begin{array}{l}40 . \\
0\end{array}$} & \multirow{2}{*}{40.0} & $\mathrm{AP}^{(3)}$ & Q & $\begin{array}{l}209 . \\
0\end{array}$ & -4.3 & -0.9 & 2.3 & - & \multirow{2}{*}{ Passa } \\
\hline & & & & & & & & $\begin{array}{l}\mathrm{AP}, \\
\mathrm{SCU}^{(2)}\end{array}$ & $\mathrm{N}, \mathrm{M}$ & $\begin{array}{l}265 . \\
5\end{array}$ & -4.4 & -1.0 & 2.5 & \begin{tabular}{|l|}
13.9 \\
\end{tabular} & \\
\hline & & Ext.Inferior & \begin{tabular}{|l|}
$\begin{array}{l}\text { Pass } \\
\mathrm{a}\end{array}$ \\
\end{tabular} & \begin{tabular}{|l} 
Pass \\
a
\end{tabular} & $\begin{array}{l}27 . \\
5\end{array}$ & $\begin{array}{l}42 \\
4\end{array}$ & 42.4 & $\begin{array}{l}\mathrm{AP}, \\
\mathrm{SCU}^{(2)}\end{array}$ & $\begin{array}{l}\mathrm{Q}, \mathrm{N}, \\
\mathrm{M}\end{array}$ & $\begin{array}{l}266 . \\
3\end{array}$ & 3.2 & 0.4 & 2.5 & $\begin{array}{l} \\
13.9 \\
\end{array}$ & Passa \\
\hline Fundação & $20 \times 20$ & $\begin{array}{l}\text { Elemento de } \\
\text { Fundação }\end{array}$ & $\begin{array}{l}\text { N.P.C } \\
\text { 1) }\end{array}$ & $\begin{array}{l}\text { N.P. } \\
1)\end{array}$ & 9.4 & $\begin{array}{l}42 \\
4\end{array}$ & 42.4 & $\begin{array}{l}\text { AP, } \\
\text { SCU }^{(2)}\end{array}$ & $\begin{array}{l}\mathrm{Q}, \mathrm{N}, \\
\mathrm{M}\end{array}$ & $\begin{array}{l}266 . \\
3\end{array}$ & 3.2 & 0.4 & 2.5 & \begin{tabular}{|l|}
13.9 \\
\end{tabular} & Passa \\
\hline \multicolumn{16}{|c|}{$\begin{array}{l}\text { Notas: } \\
\text { (1) } A \text { verificação não é necessária } \\
\text { (2) } 1.4 \cdot P P+1.4 \cdot C P+1.4 \cdot Q a \\
\text { (3) } 1.4 \cdot P P+1.4 \cdot C P\end{array}$} \\
\hline
\end{tabular}

\section{P12}

\begin{tabular}{|c|c|c|c|c|c|c|c|c|c|c|c|c|c|c|c|}
\hline \multicolumn{16}{|c|}{ Secção de betão } \\
\hline \multirow[b]{2}{*}{ Tramo } & \multirow[b]{2}{*}{$\begin{array}{l}\text { Dimens } \\
\text { ão } \\
(\mathrm{cm})\end{array}$} & \multirow[b]{2}{*}{ Posição } & \multicolumn{5}{|c|}{ Verificações } & \multicolumn{7}{|c|}{ Esforços desfavoráveis } & \multirow[b]{2}{*}{$\begin{array}{c}\text { Estad } \\
0\end{array}$} \\
\hline & & & Disp. & Arm. & $\begin{array}{c}\mathrm{Q} \\
(\% \\
)\end{array}$ & \begin{tabular}{|c|}
$\mathrm{N}$, \\
$\mathrm{M}$ \\
$(\%$ \\
)
\end{tabular} & $\begin{array}{c}\text { Apro } \\
\text { V. } \\
(\%)\end{array}$ & $\begin{array}{c}\text { Naturez } \\
a\end{array}$ & $\begin{array}{c}\text { Verif } \\
.\end{array}$ & $\stackrel{\mathrm{N}}{(\mathrm{kN})}$ & $\begin{array}{l}\mathrm{Mxx} \\
(\mathrm{kN} \cdot \\
\mathrm{m})\end{array}$ & $\begin{array}{l}\text { Myy } \\
(\mathrm{kN} . \\
\mathrm{m})\end{array}$ & $\begin{array}{c}\mathrm{Qx} \\
(\mathrm{kN} \\
)\end{array}$ & $\begin{array}{c}\text { Qy } \\
(\mathrm{kN} \\
\text { ) }\end{array}$ & \\
\hline \multirow{2}{*}{$\begin{array}{l}\text { Cobertura }(0-3.15 \\
\mathrm{m})\end{array}$} & \multirow{2}{*}{$20 \times 20$} & Ext.Superior & \begin{tabular}{|l} 
Pass \\
a
\end{tabular} & \begin{tabular}{|l} 
Pass \\
a
\end{tabular} & $\begin{array}{l}12 . \\
2\end{array}$ & $\begin{array}{l}22 . \\
9\end{array}$ & 22.9 & $\begin{array}{l}\mathrm{AP}, \\
\mathrm{SCU}^{(2)}\end{array}$ & $\begin{array}{l}\mathrm{Q}, \mathrm{N}, \\
\mathrm{M}\end{array}$ & 63.3 & 0.2 & -3.9 & 3.1 & 0.2 & Passa \\
\hline & & Ext.Inferior & $\begin{array}{l}\text { Pass } \\
a \\
\end{array}$ & \begin{tabular}{|l} 
Pass \\
a \\
\end{tabular} & $\begin{array}{l}12 . \\
2\end{array}$ & $\begin{array}{l}26 . \\
8\end{array}$ & 26.8 & $\begin{array}{l}\mathrm{AP} \\
\mathrm{SCU}^{(2)}\end{array}$ & $\begin{array}{l}\mathrm{Q}_{1} \mathrm{~N}, \\
\mathrm{M}\end{array}$ & 67.0 & -0.4 & 4.6 & 3.1 & 0.2 & Passa \\
\hline \multirow{4}{*}{$\begin{array}{l}\text { Cintamento (-1 - } 0 \\
\mathrm{~m})\end{array}$} & \multirow{4}{*}{$20 \times 20$} & \multirow{2}{*}{ Ext.Superior } & \multirow{2}{*}{$\begin{array}{l}\text { Pass } \\
\text { a }\end{array}$} & \multirow{2}{*}{$\begin{array}{l}\text { Pass } \\
\text { a }\end{array}$} & \multirow{2}{*}{$\begin{array}{l}65 . \\
1\end{array}$} & \multirow{2}{*}{$\begin{array}{l}26 . \\
4\end{array}$} & \multirow{2}{*}{65.1} & $\mathrm{AP}^{(3)}$ & Q & $\begin{array}{l}111 . \\
7\end{array}$ & -1.4 & -5.5 & $\begin{array}{l}16 . \\
6\end{array}$ & - & \multirow{2}{*}{ Passa } \\
\hline & & & & & & & & $\begin{array}{l}\mathrm{AP}, \\
\mathrm{SCU}(2)\end{array}$ & $\mathrm{N}, \mathrm{M}$ & $\begin{array}{l}134 . \\
5\end{array}$ & -1.4 & -5.3 & \begin{tabular}{|l|}
16. \\
0
\end{tabular} & - & \\
\hline & & \multirow{2}{*}{ Ext.Inferior } & \multirow{2}{*}{$\begin{array}{l}\text { Pass } \\
\text { a }\end{array}$} & \multirow{2}{*}{$\begin{array}{l}\text { Pass } \\
\text { a }\end{array}$} & \multirow{2}{*}{$\begin{array}{l}62 . \\
7\end{array}$} & \multirow{2}{*}{$\begin{array}{l}21 . \\
9\end{array}$} & \multirow{2}{*}{62.7} & $A P^{(3)}$ & Q & $\begin{array}{l}112 . \\
5\end{array}$ & 0.9 & 3.6 & $\begin{array}{l}16 . \\
6 \\
\end{array}$ & - & \multirow{2}{*}{ Passa } \\
\hline & & & & & & & & $\begin{array}{l}\mathrm{AP}, \\
\mathrm{SCU}(2) \\
\end{array}$ & $\mathrm{N}, \mathrm{M}$ & $\begin{array}{l}135 . \\
3 \\
\end{array}$ & 0.9 & 3.5 & \begin{tabular}{|l|}
16. \\
0
\end{tabular} & 4.3 & \\
\hline \multirow{2}{*}{ Fundação } & \multirow{2}{*}{$20 \times 20$} & \multirow{2}{*}{$\begin{array}{l}\text { Elemento de } \\
\text { Fundação }\end{array}$} & \multirow{2}{*}{$\begin{array}{l}\text { N.P. } \\
1)\end{array}$} & \multirow{2}{*}{$\begin{array}{l}\text { N.P. } \\
1)\end{array}$} & \multirow{2}{*}{$\begin{array}{l}11 . \\
4\end{array}$} & \multirow{2}{*}{$\begin{array}{l}21 . \\
9\end{array}$} & \multirow{2}{*}{21.9} & $A P^{(3)}$ & Q & $\begin{array}{l}112 . \\
5\end{array}$ & 0.9 & 3.6 & $\begin{array}{l}16 . \\
6\end{array}$ & - & \multirow{2}{*}{ Passa } \\
\hline & & & & & & & & $\begin{array}{l}\mathrm{AP}, \\
\mathrm{SCU}(2)\end{array}$ & $\mathrm{N}, \mathrm{M}$ & $\begin{array}{l}135 . \\
3\end{array}$ & 0.9 & 3.5 & $\begin{array}{l}16 . \\
0\end{array}$ & -4.3 & \\
\hline $\begin{array}{l}\text { Notas: } \\
\text { (1) } A \text { verificação nã } \\
\text { (2) } 1.4 \cdot P P+1.4 \cdot C P+ \\
\text { (3) } 1.4 \cdot P P+1.4 \cdot C P\end{array}$ & $\begin{array}{l}\text { necessa } \\
7 \cdot Q a\end{array}$ & & & & & & & & & & & & & & \\
\hline
\end{tabular}

\section{P13}

\begin{tabular}{|c|c|c|c|c|c|c|c|c|c|c|c|c|c|c|c|}
\hline \multicolumn{16}{|c|}{ Secção de betão } \\
\hline \multirow[b]{2}{*}{ Tramo } & \multirow[b]{2}{*}{$\begin{array}{c}\text { Dimens } \\
\text { ão } \\
(\mathrm{cm})\end{array}$} & \multirow[b]{2}{*}{ Posição } & \multicolumn{5}{|c|}{ Verificações } & \multicolumn{7}{|c|}{ Esforços desfavoráveis } & \multirow[b]{2}{*}{$\begin{array}{c}\text { Estad } \\
0\end{array}$} \\
\hline & & & Disp. & Arm. & $\begin{array}{c}\mathrm{Q} \\
(\% \\
)\end{array}$ & $\begin{array}{c}\mathrm{N}, \mathrm{M} \\
(\% \\
)\end{array}$ & $\begin{array}{c}\text { Apro } \\
\text { V. } \\
(\%)\end{array}$ & $\begin{array}{c}\text { Naturez } \\
a\end{array}$ & \begin{tabular}{|c|} 
Verif \\
$\cdot$
\end{tabular} & $\begin{array}{c}\mathrm{N} \\
(\mathrm{kN} \\
)\end{array}$ & $\begin{array}{c}M \times x \\
(k N . \\
m)\end{array}$ & $\begin{array}{c}\text { Myy } \\
(\mathrm{kN} \text {. } \\
\mathrm{m})\end{array}$ & $\begin{array}{c}\text { Qx } \\
(\mathrm{kN} \\
) \\
\end{array}$ & $\begin{array}{c}\text { Qy } \\
(k N)\end{array}$ & \\
\hline $\begin{array}{l}\text { Cintamento }(-1-0 \\
\mathrm{m})\end{array}$ & $20 \times 20$ & Ext.Superior & $\begin{array}{l}\text { Pass } \\
\text { a }\end{array}$ & $\begin{array}{l}\text { Pass } \\
\text { a }\end{array}$ & $\begin{array}{l}98 . \\
6\end{array}$ & $\begin{array}{l}26 . \\
5\end{array}$ & 98.6 & $\begin{array}{l}\mathrm{AP} \\
\mathrm{SCU}\end{array}$ & $\begin{array}{l}\mathrm{Q}, \mathrm{N}, \\
\mathrm{M}\end{array}$ & $\begin{array}{l}39 . \\
7\end{array}$ & -3.7 & -4.3 & $\begin{array}{l}19 . \\
0\end{array}$ & $\begin{array}{l}- \\
16.8\end{array}$ & Passa \\
\hline
\end{tabular}




\section{Pontifícia Universidade C Católica}

DO RIO DE JANEIRO

\begin{tabular}{|c|c|c|c|c|c|c|c|c|c|c|c|c|c|c|c|}
\hline \multicolumn{16}{|c|}{ Secção de betão } \\
\hline \multirow{3}{*}{ Tramo } & \multirow{3}{*}{$\begin{array}{c}\text { Dimens } \\
\text { ão } \\
(\mathrm{cm})\end{array}$} & \multirow[b]{2}{*}{ Posição } & \multicolumn{5}{|c|}{ Verificações } & \multicolumn{7}{|c|}{ Esforços desfavoráveis } & \multirow[b]{2}{*}{$\begin{array}{c}\text { Estad } \\
0\end{array}$} \\
\hline & & & Disp. & Arm. & \begin{tabular}{|c|}
$\mathrm{Q}$ \\
$(\%$ \\
) \\
\end{tabular} & $\begin{array}{c}\mathrm{N}, \mathrm{M} \\
(\% \\
)\end{array}$ & $\begin{array}{c}\text { Apro } \\
\text { V. } \\
(\%)\end{array}$ & $\begin{array}{c}\text { Naturez } \\
a\end{array}$ & $\begin{array}{c}\text { Verif } \\
.\end{array}$ & $\begin{array}{c}\mathrm{N} \\
(\mathrm{kN} \\
)\end{array}$ & $\begin{array}{c}M x x \\
(k N . \\
m)\end{array}$ & $\begin{array}{c}\text { Myy } \\
(\mathrm{kN} . \\
\mathrm{m})\end{array}$ & $\begin{array}{c}\text { Qx } \\
(\mathrm{kN} \\
) \\
\end{array}$ & $\begin{array}{c}\text { Qy } \\
(k N)\end{array}$ & \\
\hline & & Ext.Inferior & \begin{tabular}{|l|} 
Pass \\
a
\end{tabular} & \begin{tabular}{|l|} 
Pass \\
a
\end{tabular} & $\begin{array}{l}98 . \\
6 \\
\end{array}$ & $\begin{array}{l}40 . \\
6 \\
\end{array}$ & 98.6 & $\begin{array}{l}\mathrm{AP}_{1} \\
\mathrm{SCU}^{(2)}\end{array}$ & $\begin{array}{l}\mathrm{Q}, \mathrm{N}, \\
\mathrm{M}\end{array}$ & $\begin{array}{l}40 . \\
5\end{array}$ & 5.5 & 6.1 & $\begin{array}{l}19 . \\
0\end{array}$ & \begin{tabular}{|l|} 
\\
16.8 \\
\end{tabular} & Passa \\
\hline Fundação & $20 \times 20$ & $\begin{array}{l}\text { Elemento de } \\
\text { Fundação }\end{array}$ & $\begin{array}{l}\text { N.P. } \\
1)\end{array}$ & $\begin{array}{l}\text { N.P. ( } \\
1)\end{array}$ & $\begin{array}{l}16 . \\
8\end{array}$ & $\begin{array}{l}40 . \\
6\end{array}$ & 40.6 & $\begin{array}{l}\mathrm{AP}, \\
\mathrm{SCU}{ }^{(2)}\end{array}$ & $\begin{array}{l}Q, N, \\
M\end{array}$ & $\begin{array}{l}40 . \\
5\end{array}$ & 5.5 & 6.1 & $\begin{array}{l}19 . \\
0\end{array}$ & 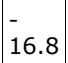 & Passa \\
\hline $\begin{array}{l}\text { Notas: } \\
\text { (1) A verif } \\
\text { (2) } 1.4 \cdot P P\end{array}$ & $\begin{array}{l}\text { é necess } \\
4 \cdot Q a\end{array}$ & & & & & & & & & & & & & & \\
\hline
\end{tabular}

\section{P14}

\begin{tabular}{|c|c|c|c|c|c|c|c|c|c|c|c|c|c|c|c|}
\hline \multicolumn{16}{|c|}{ Secção de betão } \\
\hline \multirow[b]{2}{*}{ Tramo } & \multirow[b]{2}{*}{$\begin{array}{c}\text { Dimens } \\
\text { ão } \\
(\mathrm{cm})\end{array}$} & \multirow[b]{2}{*}{ Posição } & \multicolumn{5}{|c|}{ Verificações } & \multicolumn{7}{|c|}{ Esforços desfavoráveis } & \multirow[b]{2}{*}{$\begin{array}{c}\text { Estad } \\
0\end{array}$} \\
\hline & & & Disp. & Arm. & $\begin{array}{c}\mathrm{Q} \\
(\% \\
)\end{array}$ & \begin{tabular}{|c|}
$\mathrm{N}$ \\
$\mathrm{M}$ \\
$(\%$ \\
)
\end{tabular} & $\begin{array}{c}\text { Apro } \\
\text { V. } \\
(\%)\end{array}$ & $\begin{array}{c}\text { Naturez } \\
a\end{array}$ & $\begin{array}{c}\text { Verif } \\
\cdot\end{array}$ & $\begin{array}{c}\mathrm{N} \\
(\mathrm{kN} \\
)\end{array}$ & $\begin{array}{c}M x x \\
(k N . \\
m)\end{array}$ & $\begin{array}{l}\text { Myy } \\
(\mathrm{kN} . \\
\mathrm{m})\end{array}$ & $\begin{array}{c}\mathrm{Qx} \\
(\mathrm{kN})\end{array}$ & $\begin{array}{c}\text { Qy } \\
(\mathrm{kN} \\
\text { ) }\end{array}$ & \\
\hline \multirow{2}{*}{$\begin{array}{l}\text { Cobertura (0 - } 3.15 \\
\text { m) }\end{array}$} & \multirow{2}{*}{$20 \times 20$} & Ext.Superior & $\begin{array}{l}\text { Pass } \\
\text { a }\end{array}$ & \begin{tabular}{|l|} 
Pass \\
a
\end{tabular} & 2.9 & 8.5 & 8.5 & $\begin{array}{l}\mathrm{AP}_{1} \\
\mathrm{SCU}^{(2)}\end{array}$ & $\begin{array}{l}\mathrm{Q}, \mathrm{N}, \\
\mathrm{M}\end{array}$ & $\begin{array}{l}27 . \\
0\end{array}$ & 0.2 & 1.1 & -0.7 & 0.2 & Passa \\
\hline & & Ext.Inferior & \begin{tabular}{|l} 
Pass \\
a
\end{tabular} & \begin{tabular}{|l|} 
Pass \\
a
\end{tabular} & 2.5 & 8.6 & 8.6 & $\begin{array}{l}\mathrm{AP}, \\
\mathrm{SCU}^{(2)}\end{array}$ & $\begin{array}{l}\mathrm{Q}, \mathrm{N}, \\
\mathrm{M}\end{array}$ & $\begin{array}{l}30 . \\
7\end{array}$ & -0.3 & -0.9 & -0.7 & 0.2 & Passa \\
\hline \multirow{2}{*}{$\begin{array}{l}\text { Cintamento }(-1-0 \\
\mathrm{m})\end{array}$} & \multirow{2}{*}{$20 \times 20$} & Ext.Superior & $\begin{array}{l}\text { Pass } \\
\text { a }\end{array}$ & $\begin{array}{l}\text { Pass } \\
\text { a }\end{array}$ & $\begin{array}{l}95 . \\
3\end{array}$ & $\begin{array}{l}34 . \\
7\end{array}$ & 95.3 & $\begin{array}{l}\text { AP, } \\
\text { SCU(2) }\end{array}$ & $\begin{array}{l}\mathrm{Q}, \mathrm{N}, \\
\mathrm{M}\end{array}$ & $\begin{array}{l}73 . \\
8\end{array}$ & 5.7 & 5.4 & $\begin{array}{l}- \\
17.4\end{array}$ & $\begin{array}{l}17 . \\
2\end{array}$ & Passa \\
\hline & & Ext.Inferior & $\begin{array}{l}\text { Pass } \\
\text { a }\end{array}$ & \begin{tabular}{|l|} 
Pass \\
a
\end{tabular} & $\begin{array}{l}95 . \\
3\end{array}$ & $\begin{array}{l}24 . \\
7\end{array}$ & 95.3 & $\begin{array}{l}\mathrm{AP}, \\
\mathrm{SCU}(2)\end{array}$ & $\begin{array}{l}\mathrm{Q}, \mathrm{N}, \\
\mathrm{M}\end{array}$ & $\begin{array}{l}74 . \\
6\end{array}$ & -3.7 & -4.2 & \begin{tabular}{|l|}
17.4 \\
\end{tabular} & $\begin{array}{l}17 . \\
2\end{array}$ & Passa \\
\hline Fundação & $20 \times 20$ & $\begin{array}{l}\text { Elemento de } \\
\text { Fundação }\end{array}$ & $\begin{array}{l}\text { N.P. } \\
1)\end{array}$ & \begin{tabular}{|l|} 
N.P.C \\
$1)$
\end{tabular} & $\begin{array}{l}16 . \\
3\end{array}$ & $\begin{array}{l}24 . \\
7\end{array}$ & 24.7 & $\begin{array}{l}\text { AP, } \\
S C U^{(2)}\end{array}$ & $\begin{array}{l}\mathrm{Q}, \mathrm{N}, \\
\mathrm{M}\end{array}$ & $\begin{array}{l}74 . \\
6\end{array}$ & -3.7 & -4.2 & \begin{tabular}{|l|}
- \\
17.4
\end{tabular} & $\begin{array}{l}17 . \\
2\end{array}$ & Passa \\
\hline $\begin{array}{l}\text { Notas: } \\
\text { (1) A verificacão nâ } \\
\text { (2) } 1.4 \cdot P P+1.4 \cdot C P\end{array}$ & $\begin{array}{l}\text { necessa } \\
\text { na }\end{array}$ & & & & & & & & & & & & & & \\
\hline
\end{tabular}

\section{P15}

\begin{tabular}{|c|c|c|c|c|c|c|c|c|c|c|c|c|c|c|c|}
\hline \multicolumn{16}{|c|}{ Secção de betão } \\
\hline \multirow[b]{2}{*}{ Tramo } & \multirow[b]{2}{*}{$\begin{array}{c}\text { Dimens } \\
\text { ão } \\
(\mathrm{cm})\end{array}$} & \multirow[b]{2}{*}{ Posição } & \multicolumn{5}{|c|}{ Verificações } & \multicolumn{7}{|c|}{ Esforços desfavoráveis } & \multirow[b]{2}{*}{$\begin{array}{c}\text { Estad } \\
0\end{array}$} \\
\hline & & & Disp. & Arm. & $\begin{array}{c}\mathrm{Q} \\
(\% \\
)\end{array}$ & \begin{tabular}{|c|}
$\mathrm{N}$ \\
$\mathrm{M}$ \\
$(\%$ \\
)
\end{tabular} & $\begin{array}{c}\text { Apro } \\
\text { V. } \\
(\%)\end{array}$ & $\begin{array}{c}\text { Naturez } \\
\text { a }\end{array}$ & $\begin{array}{c}\text { Verif } \\
.\end{array}$ & $\stackrel{\mathrm{N}}{(\mathrm{kN})}$ & $\begin{array}{c}M x x \\
(k N . \\
m)\end{array}$ & $\begin{array}{c}\text { Myy } \\
(\mathrm{kN} \text {. } \\
\mathrm{m})\end{array}$ & $\begin{array}{c}\text { Qx } \\
(k N)\end{array}$ & $\begin{array}{c}\text { Qy } \\
\text { (kN } \\
\text { ) }\end{array}$ & \\
\hline \multirow{2}{*}{$\begin{array}{l}\text { Cobertura }(0-3.15 \\
\mathrm{m})\end{array}$} & \multirow{2}{*}{$25 \times 20$} & Ext.Superior & $\begin{array}{l}\text { Pass } \\
\text { a }\end{array}$ & $\begin{array}{l}\text { Pass } \\
\text { a }\end{array}$ & 1.2 & $\begin{array}{l}16 . \\
0\end{array}$ & 16.0 & $\begin{array}{l}\mathrm{AP} \\
\mathrm{SCU}^{(2)}\end{array}$ & $\begin{array}{l}\mathrm{Q}, \mathrm{N}, \\
\mathrm{M}\end{array}$ & 85.5 & 0.7 & 0.0 & 0.2 & 0.7 & Passa \\
\hline & & Ext.Inferior & \begin{tabular}{|l|} 
Pass \\
a
\end{tabular} & \begin{tabular}{|l|} 
Pass \\
a
\end{tabular} & 1.2 & $\begin{array}{l}17 . \\
5\end{array}$ & 17.5 & $\begin{array}{l}\mathrm{AP}, \\
\mathrm{SCU}\end{array}$ & $\begin{array}{l}\mathrm{Q}, \mathrm{N}, \\
\mathrm{M}\end{array}$ & 90.1 & -1.3 & 0.5 & 0.2 & 0.7 & Passa \\
\hline \multirow{2}{*}{$\begin{array}{l}\text { Cintamento (-1 - } 0 \\
\text { m) }\end{array}$} & \multirow{2}{*}{$25 \times 20$} & Ext.Superior & \begin{tabular}{|l|} 
Pass \\
a
\end{tabular} & $\begin{array}{l}\text { Pass } \\
\text { a }\end{array}$ & $\begin{array}{l}91 . \\
3\end{array}$ & $\begin{array}{l}36 . \\
7\end{array}$ & 91.3 & $\begin{array}{l}\mathrm{AP} \\
\mathrm{SCU}^{(2)}\end{array}$ & $\begin{array}{l}\mathrm{Q}, \mathrm{N}, \\
\mathrm{M}\end{array}$ & $\begin{array}{l}180 . \\
2\end{array}$ & 9.0 & 4.8 & $-\overline{15.9}$ & $\begin{array}{l}27 \\
6\end{array}$ & Passa \\
\hline & & Ext.Inferior & \begin{tabular}{|l|} 
Pass \\
$\mathrm{a}$
\end{tabular} & \begin{tabular}{|l|} 
Pass \\
a
\end{tabular} & $\begin{array}{l}89 . \\
6\end{array}$ & $\begin{array}{l}29 . \\
7\end{array}$ & 89.6 & $\begin{array}{l}\mathrm{AP}, \\
\mathrm{SCU}(2)\end{array}$ & $\begin{array}{l}\mathrm{Q}, \mathrm{N}, \\
\mathrm{M}\end{array}$ & $\begin{array}{l}181 . \\
1\end{array}$ & -6.2 & -3.9 & $\begin{array}{l}- \\
15.9 \\
\end{array}$ & $\begin{array}{l}27 \\
6\end{array}$ & Passa \\
\hline Fundação & $25 \times 20$ & $\begin{array}{l}\text { Elemento de } \\
\text { Fundação }\end{array}$ & $\begin{array}{l}\text { N.P. ( } \\
1)\end{array}$ & $\begin{array}{l}\text { N.P. } \\
1)\end{array}$ & $\begin{array}{l}16 . \\
8\end{array}$ & $\begin{array}{l}29 . \\
7\end{array}$ & 29.7 & $\begin{array}{l}\mathrm{AP} \\
\mathrm{SCU}^{(2)}\end{array}$ & $\begin{array}{l}Q, N, \\
M\end{array}$ & $\begin{array}{l}181 . \\
1\end{array}$ & -6.2 & -3.9 & $-\overline{15.9}$ & $\begin{array}{l}27 \\
6\end{array}$ & Passa \\
\hline
\end{tabular}




\section{Pontifícia Universidade Católloa}

P16

\begin{tabular}{|c|c|c|c|c|c|c|c|c|c|c|c|c|c|c|c|}
\hline \multicolumn{16}{|c|}{ Secção de betão } \\
\hline \multirow[b]{2}{*}{ Tramo } & \multirow[b]{2}{*}{$\begin{array}{c}\text { Dimens } \\
\text { ão } \\
(\mathrm{cm})\end{array}$} & \multirow[b]{2}{*}{ Posição } & \multicolumn{5}{|c|}{ Verificações } & \multicolumn{7}{|c|}{ Esforços desfavoráveis } & \multirow[b]{2}{*}{$\begin{array}{c}\text { Estad } \\
0\end{array}$} \\
\hline & & & Disp. & Arm. & $\begin{array}{c}\mathrm{Q} \\
(\% \\
)\end{array}$ & $\begin{array}{c}\mathrm{N}, \mathrm{M} \\
(\% \\
)\end{array}$ & \begin{tabular}{|c|} 
Apro \\
V. \\
$(\%)$
\end{tabular} & $\begin{array}{c}\text { Naturez } \\
\text { a }\end{array}$ & $\begin{array}{c}\text { Verif } \\
.\end{array}$ & $\begin{array}{c}\mathrm{N} \\
(\mathrm{kN} \\
)\end{array}$ & $\begin{array}{c}M x x \\
(k N . \\
m)\end{array}$ & $\begin{array}{c}\text { Myy } \\
(\mathrm{kN} . \\
\mathrm{m})\end{array}$ & $\begin{array}{c}\text { Qx } \\
(\mathrm{kN} \\
) \\
\end{array}$ & \begin{tabular}{|c|} 
Qy \\
$(\mathrm{kN}$ \\
)
\end{tabular} & \\
\hline \multirow{2}{*}{$\begin{array}{l}\text { Cobertura (0 - } 3.15 \\
\text { m) }\end{array}$} & \multirow{2}{*}{$20 \times 20$} & Ext.Superior & \begin{tabular}{|l} 
Pass \\
a
\end{tabular} & \begin{tabular}{|l|} 
Pass \\
a
\end{tabular} & 8.6 & $\begin{array}{l}15 . \\
6 \\
\end{array}$ & 15.6 & $\begin{array}{l}\mathrm{AP} \\
\mathrm{SCU}^{(2)} \\
\end{array}$ & $\begin{array}{l}Q, N, \\
M\end{array}$ & $\begin{array}{l}32 . \\
1\end{array}$ & 0.8 & -2.5 & 2.1 & 0.9 & Passa \\
\hline & & Ext.Inferior & \begin{tabular}{|l} 
Pass \\
a
\end{tabular} & \begin{tabular}{|l|} 
Pass \\
a
\end{tabular} & 8.9 & $\begin{array}{l}21 . \\
2\end{array}$ & 21.2 & $\begin{array}{l}\mathrm{AP} \\
\mathrm{SCU} \\
\end{array}$ & $\begin{array}{l}Q, N, \\
M\end{array}$ & $\begin{array}{l}35 . \\
8\end{array}$ & -1.5 & 3.2 & 2.1 & 0.9 & Passa \\
\hline \multirow{2}{*}{$\begin{array}{l}\text { Cintamento }(-1-0 \\
\mathrm{m})\end{array}$} & \multirow{2}{*}{$20 \times 20$} & Ext.Superior & $\begin{array}{l}\text { Pass } \\
\text { a }\end{array}$ & \begin{tabular}{|l|} 
Pass \\
a
\end{tabular} & $\begin{array}{l}39 . \\
4\end{array}$ & $\begin{array}{l}39 . \\
6\end{array}$ & 39.6 & $\begin{array}{l}\mathrm{AP} \\
\mathrm{SCU}^{(2)}\end{array}$ & $\begin{array}{l}Q, N, \\
M\end{array}$ & $\begin{array}{l}84 . \\
5\end{array}$ & 5.2 & -7.3 & $\begin{array}{l}22 . \\
0\end{array}$ & $\begin{array}{l}16 . \\
4\end{array}$ & Passa \\
\hline & & Ext.Inferior & \begin{tabular}{|l} 
Pass \\
a \\
\end{tabular} & \begin{tabular}{|l|} 
Pass \\
a
\end{tabular} & $\begin{array}{l}39 . \\
4\end{array}$ & $\begin{array}{l}26 . \\
8\end{array}$ & 39.4 & $\begin{array}{l}\mathrm{AP} \\
\mathrm{SCU}^{(2)}\end{array}$ & $\begin{array}{l}\mathrm{Q}, \mathrm{N}, \\
\mathrm{M}\end{array}$ & $\begin{array}{l}85 . \\
2\end{array}$ & -3.8 & 4.8 & $\begin{array}{l}22 . \\
0\end{array}$ & $\begin{array}{l}16 . \\
4\end{array}$ & Passa \\
\hline Fundação & $20 \times 20$ & $\begin{array}{l}\text { Elemento de } \\
\text { Fundação }\end{array}$ & $\begin{array}{l}\text { N.P. } \\
1)\end{array}$ & $\begin{array}{l}\text { N.P. } \\
1)\end{array}$ & $\begin{array}{l}18 . \\
3\end{array}$ & $\begin{array}{l}26 . \\
8\end{array}$ & 26.8 & $\begin{array}{l}\mathrm{AP} \\
\mathrm{SCU}^{(2)}\end{array}$ & $\begin{array}{l}Q, N, \\
M\end{array}$ & $\begin{array}{l}85 . \\
2\end{array}$ & -3.8 & 4.8 & $\begin{array}{l}22 . \\
0\end{array}$ & $\begin{array}{l}16 . \\
4\end{array}$ & Passa \\
\hline
\end{tabular}

\subsection{5 - VERIFICAÇÃO DAS VIGAS}

\section{Cintamento}

\begin{tabular}{|c|c|c|c|c|c|c|c|c|c|c|c|c|c|c|c|c|}
\hline \multirow{2}{*}{ Vigas } & \multicolumn{15}{|c|}{ VERIFICAÇÕES DE RESISTÊNCIA (ABNT NBR 6118:2014) } & \multirow{2}{*}{ Estado } \\
\hline & Disp. & Arm. & Q & $\mathrm{N}, \mathrm{M}$ & $T_{c}$ & $\mathrm{~T}_{\mathrm{st}}$ & $T_{s l}$ & $\mathrm{TNM}_{\mathrm{x}}$ & $\mathrm{TV}_{\mathrm{x}}$ & $\mathrm{TV}_{\mathrm{y}}$ & TV $x S_{t}$ & TVYSt & T,Disp.s1 & T,Geom.st & T,Arm.st & \\
\hline a: P1 - V 11 & assa & assa & $\begin{array}{l}.257 \mathrm{~m}^{\prime} \\
=19.7\end{array}$ & $\begin{array}{l}1 \\
\eta\end{array}=33.1$ & N.P. ${ }^{(1)}$ & N.P. ${ }^{(1)}$ & N.P. ${ }^{(1)}$ & N.P. ${ }^{(2)}$ & N.P. ${ }^{(1)}$ & N.P. ${ }^{(1)}$ & N.P. ${ }^{(1)}$ & N.P. ${ }^{(1)}$ & & N.P. ${ }^{(1)}$ & & \\
\hline b: V $11-P 2$ & assa & assa & $\begin{array}{l}1 \\
\eta \\
\eta=22.019 \mathrm{~m}\end{array}$ & $\begin{array}{l}\prime 0.938 \mathrm{~m}^{\prime} \\
\eta=38.4\end{array}$ & N.P. ${ }^{(1)}$ & N.P. ${ }^{(1)}$ & .P. ${ }^{(1)}$ & N.P. (2) & N.P. ${ }^{(1)}$ & N.P. ${ }^{(1)}$ & N.P. ${ }^{(1)}$ & N.P. ${ }^{(1)}$ & & & & \\
\hline c: P2 - P3 & assa & assa & $\begin{array}{c}0.257 \mathrm{~m}^{\prime} \\
\eta=7.9\end{array}$ & $\begin{array}{c}\text { 'P2' } \\
\eta=30.4\end{array}$ & N.P. ${ }^{(1)}$ & N.P. ${ }^{(1)}$ & N.P. ${ }^{(1)}$ & N.P. (2) & N.P. ${ }^{(1)}$ & N.P. ${ }^{(1)}$ & N.P. ${ }^{(1)}$ & N.P. ${ }^{(1)}$ & N.P. ${ }^{(1)}$ & & N.P. ${ }^{(1)}$ & \\
\hline a: P5 - P6 & assa & assa & $\begin{array}{l}0.207 \mathrm{~m}^{\prime} \\
\eta=38.6\end{array}$ & $\begin{array}{c}\text { 'P5' } \\
\eta=81.5\end{array}$ & N.P. ${ }^{(1)}$ & N.P. ${ }^{(1)}$ & N.P. ${ }^{(1)}$ & N.P. ${ }^{(2)}$ & N.P. ${ }^{(1)}$ & N.P. ${ }^{(1)}$ & N.P. ${ }^{(1)}$ & N.P. ${ }^{(1)}$ & N.P. ${ }^{(1)}$ & N.P. ${ }^{(1)}$ & N.P. ${ }^{(1)}$ & \\
\hline a: P7 - P8 & Passa & Passa & $\begin{array}{l}.044 \mathrm{~m}^{\prime} \\
=39.5\end{array}$ & & N.P. ${ }^{(1)}$ & N.P. ${ }^{(1)}$ & N.P. ${ }^{(1)}$ & N.P. ${ }^{(2)}$ & N.P. ${ }^{(1)}$ & N.P. ${ }^{(1)}$ & N.P. ${ }^{(1)}$ & N.P. ${ }^{(1)}$ & N.P. ${ }^{(1)}$ & N.P. ${ }^{(1)}$ & N.P. ${ }^{(1)}$ & \\
\hline a: P10 - P11 & Passa & Passa & & & N.P. ${ }^{(1)}$ & N.P. ${ }^{(1)}$ & N.P. ${ }^{(1)}$ & N.P. ${ }^{(2)}$ & N.P. ${ }^{(1)}$ & N.P. ${ }^{(1)}$ & N.P. ${ }^{(1)}$ & N.P. ${ }^{(1)}$ & N.P. ${ }^{(1)}$ & N.P. ${ }^{(1)}$ & N.P. ${ }^{(1)}$ & \\
\hline b: P11 - P12 & assa & Passa & & & N.P. ${ }^{(1)}$ & N.P. ${ }^{(1)}$ & N.P. ${ }^{(1)}$ & N.P. (2) & N.P. ${ }^{(1)}$ & N.P. ${ }^{(1)}$ & N.P. ${ }^{(1)}$ & N.P. ${ }^{(1)}$ & & & & \\
\hline $\mathrm{b}: \mathrm{V} 14-\mathrm{P} 13$ & assa & Passa & & & N.P. ${ }^{(1)}$ & N.P. (1) & N.P. ${ }^{(1)}$ & N.P. (2) & N.P. ${ }^{(1)}$ & N.P. ${ }^{(1)}$ & N.P. ${ }^{(1)}$ & N.P. ${ }^{(1)}$ & & & & \\
\hline a: P14 - P15 & assa & Passa & & & N.P. ${ }^{(1)}$ & N.P. (1) & N.P. ${ }^{(1)}$ & N.P. (2) & N.P. ${ }^{(1)}$ & N.P. ${ }^{(1)}$ & N.P. ${ }^{(1)}$ & N.P. ${ }^{(1)}$ & & & & \\
\hline b: P15 - V 14 & assa & Passa & & & N.P. ${ }^{(1)}$ & N.P.(1) & N.P. ${ }^{(1)}$ & N.P. (2) & N.P. (1) & N.P. ${ }^{(1)}$ & N.P. ${ }^{(1)}$ & N.P. ${ }^{(1)}$ & & & & \\
\hline c: V 14 - V 16 & Passa & Passa & $\begin{array}{r}00 \mathrm{~m}^{\prime} \\
=12.3 \\
\end{array}$ & & N.P. ${ }^{(1)}$ & N.P. ${ }^{(1)}$ & N.P. ${ }^{(1)}$ & N.P. (2) & N.P. ${ }^{(1)}$ & N.P. ${ }^{(1)}$ & N.P. ${ }^{(1)}$ & N.P. ${ }^{(1)}$ & & & & \\
\hline $\mathrm{d}: \mathrm{V} 16-\mathrm{P} 16$ & assa & Passa & $\begin{array}{l}1.619 \mathrm{~m}^{\prime} \\
\eta=30.6\end{array}$ & & N.P. ${ }^{(1)}$ & N.P. ${ }^{(1)}$ & N.P. ${ }^{(1)}$ & N.P. (2) & N.P. (1) & N.P. ${ }^{(1)}$ & N.P. ${ }^{(1)}$ & N.P. ${ }^{(1)}$ & & & & \\
\hline a: P14 - P10 & Passa & Passa & $\begin{array}{l}4.044 \mathrm{~m}^{\prime} \\
\eta=25.7\end{array}$ & & N.P. ${ }^{(1)}$ & N.P. ${ }^{(1)}$ & N.P. ${ }^{(1)}$ & N.P. (2) & N.P. ${ }^{(1)}$ & N.P. ${ }^{(1)}$ & N.P. ${ }^{(1)}$ & N.P. ${ }^{(1)}$ & N.P. ${ }^{(1)}$ & & & \\
\hline b: P10 - P7 & Passa & Passa & $\begin{array}{l}2.269 \mathrm{~m}^{\prime} \\
\eta=14.3\end{array}$ & & N.P. ${ }^{(1)}$ & N.P. ${ }^{(1)}$ & N.P. ${ }^{(1)}$ & N.P. (2) & N.P. ${ }^{(1)}$ & N.P. ${ }^{(1)}$ & N.P. ${ }^{(1)}$ & N.P. ${ }^{(1)}$ & & & & \\
\hline c: P7 - P1 & Passa & Passa & & & N.P. ${ }^{(1)}$ & N.P. ${ }^{(1)}$ & N.P. ${ }^{(1)}$ & N.P. (2) & N.P. ${ }^{(1)}$ & N.P. ${ }^{(1)}$ & N.P. ${ }^{(1)}$ & N.P. ${ }^{(1)}$ & N.P. ${ }^{(1)}$ & N.P. ${ }^{(1)}$ & N.P. ${ }^{(1)}$ & \\
\hline a: P15 - V 7 & Passa & Passa & $\begin{array}{l}10.207 \mathrm{~m}^{\prime} \\
\eta=36.6 \\
\end{array}$ & & N.P. ${ }^{(1)}$ & N.P.(1) & N.P. ${ }^{(1)}$ & N.P. ${ }^{(2)}$ & N.P. ${ }^{(1)}$ & N.P. ${ }^{(1)}$ & N.P. ${ }^{(1)}$ & N.P. ${ }^{(1)}$ & N.P. ${ }^{(1)}$ & N.P. ${ }^{(1)}$ & N.P. ${ }^{(1)}$ & \\
\hline b: V $7-P 11$ & Passa & Passa & $\begin{array}{l}2.294 \mathrm{~m}^{\prime} \\
\eta=32.4\end{array}$ & $\begin{array}{c}\text { 'V 7' } \\
\eta=71.0\end{array}$ & N.P. ${ }^{(1)}$ & N.P. ${ }^{(1)}$ & N.P. ${ }^{(1)}$ & N.P. (2) & N.P. (1) & N.P. ${ }^{(1)}$ & N.P. ${ }^{(1)}$ & N.P. ${ }^{(1)}$ & N.P. ${ }^{(1)}$ & N.P. ${ }^{(1)}$ & N.P. ${ }^{(1)}$ & \\
\hline c: P11 - P8 & Passa & Passa & $\begin{array}{l}10.207 \mathrm{~m}^{\prime} \\
\eta=22.3 \\
\end{array}$ & $\begin{array}{c}\text { 'P11' } \\
\eta=55.4\end{array}$ & N.P. ${ }^{(1)}$ & N.P. ${ }^{(1)}$ & N.P. ${ }^{(1)}$ & N.P. (2) & N.P. ${ }^{(1)}$ & N.P. ${ }^{(1)}$ & N.P. ${ }^{(1)}$ & N.P. ${ }^{(1)}$ & N.P. ${ }^{(1)}$ & N.P. ${ }^{(1)}$ & N.P. ${ }^{(1)}$ & \\
\hline d: P8 - P5 & Passa & Passa & $\begin{array}{c}0.819 \mathrm{~m}^{\prime} \\
\eta=7.0\end{array}$ & & N.P. ${ }^{(1)}$ & N.P. ${ }^{(1)}$ & N.P. ${ }^{(1)}$ & N.P. (2) & N.P. ${ }^{(1)}$ & N.P. ${ }^{(1)}$ & N.P. ${ }^{(1)}$ & N.P. ${ }^{(1)}$ & N.P. ${ }^{(1)}$ & N.P. ${ }^{(1)}$ & N.P. ${ }^{(1)}$ & \\
\hline a: P5 - P2 & Passa & Passa & $\begin{array}{l}\prime 0.207 \mathrm{~m}^{\prime} \\
\eta=15.6\end{array}$ & $\begin{array}{c}\text { 'P5' } \\
\eta=20.2\end{array}$ & N.P. ${ }^{(1)}$ & N.P. ${ }^{(1)}$ & N.P. ${ }^{(1)}$ & N.P. (2) & N.P. (1) & N.P. ${ }^{(1)}$ & N.P. ${ }^{(1)}$ & N.P. ${ }^{(1)}$ & N.P. ${ }^{(1)}$ & N.P. ${ }^{(1)}$ & N.P. ${ }^{(1)}$ & $\begin{array}{c}\text { PASSA } \\
\eta=20.2\end{array}$ \\
\hline
\end{tabular}




\begin{tabular}{|c|c|c|c|c|c|c|c|c|c|c|c|c|c|c|c|c|}
\hline \multirow{2}{*}{ Vigas } & \multicolumn{15}{|c|}{ VERIFICAÇÕES DE RESISTÊNCIA (ABNT NBR 6118:2014) } & \multirow{2}{*}{ Estado } \\
\hline & Disp. & Arm. & Q & $\mathrm{N}, \mathrm{M}$ & $\mathrm{T}_{\mathrm{c}}$ & $\mathrm{T}_{\text {st }}$ & $\mathrm{T}_{\mathrm{sl}}$ & TNM $_{x}$ & $\mathrm{TV}_{\mathrm{x}}$ & $\mathrm{TV}_{\mathrm{y}}$ & TV $\mathrm{SSt}_{\mathrm{t}}$ & TV Y St & T,Disp.sl & T,Geom.st & $\mathrm{t}$ T,Arm.st & \\
\hline a: $P 9-V 3$ & Passa & Passa & $\begin{array}{l}' 0.207 \mathrm{~m}^{\prime} \\
\eta=17.7\end{array}$ & $\begin{array}{c}\text { 'P9' } \\
\eta=25.8\end{array}$ & N.P. ${ }^{(1)}$ & N.P. ${ }^{(1)}$ & N.P. ${ }^{(1)}$ & N.P. (2) & N.P. ${ }^{(1)}$ & N.P. ${ }^{(1)}$ & N.P. ${ }^{(1)}$ & N.P. ${ }^{(1)}$ & N.P. ${ }^{(1)}$ & N.P. ${ }^{(1)}$ & N.P. ${ }^{(1)}$ & $\begin{array}{c}\text { PASSA } \\
\eta=25.8\end{array}$ \\
\hline a: P16 - P12 & Passa & Passa & $\begin{array}{c}14.044 \mathrm{~m}^{\prime} \\
\eta=25.7\end{array}$ & $\begin{array}{l}\text { '3.946 m' } \\
\eta=51.2\end{array}$ & N.P. ${ }^{(1)}$ & N.P. ${ }^{(1)}$ & N.P. ${ }^{(1)}$ & N.P. (2) & N.P. ${ }^{(1)}$ & N.P. ${ }^{(1)}$ & N.P. ${ }^{(1)}$ & N.P. ${ }^{(1)}$ & N.P. ${ }^{(1)}$ & N.P. ${ }^{(1)}$ & N.P. ${ }^{(1)}$ & $\begin{array}{c}\text { PASSA } \\
\eta=51.2\end{array}$ \\
\hline b: P12 - P6 & Passa & Passa & $\begin{array}{l}\prime 0.207 \mathrm{~m}^{\prime} \\
\eta=22.5\end{array}$ & $\begin{array}{c}\text { 'P12' } \\
\eta=49.8\end{array}$ & N.P. ${ }^{(1)}$ & N.P. ${ }^{(1)}$ & N.P. ${ }^{(1)}$ & N.P. (2) & N.P. ${ }^{(1)}$ & N.P. ${ }^{(1)}$ & N.P. ${ }^{(1)}$ & N.P. ${ }^{(1)}$ & N.P. ${ }^{(1)}$ & N.P. ${ }^{(1)}$ & N.P. ${ }^{(1)}$ & $\begin{array}{c}\text { PASSA } \\
\eta=49.8\end{array}$ \\
\hline
\end{tabular}

\begin{tabular}{|c|c|c|c|c|c|c|c|c|c|c|c|c|c|c|c|c|c|}
\hline \multirow{2}{*}{ Vigas } & \multicolumn{16}{|c|}{ VERIFICAÇÕES DE RESISTÊNCIA (ABNT NBR 6118:2014) } & \multirow{2}{*}{ Estado } \\
\hline & Disp. & Arm. & Q & $\mathrm{N}, \mathrm{M}$ & $\mathrm{T}_{\mathrm{c}}$ & $\mathrm{T}_{\mathrm{st}}$ & $T_{\text {sl }}$ & $\mathrm{TNM}_{\mathrm{x}}$ & $\mathrm{TV}_{\mathrm{x}}$ & $\mathrm{TV}_{\mathrm{y}}$ & TVxSt & TVYSt & T,Disp.s! & T,Geom.st & T,Arm.st & - & \\
\hline a: V 9 - P4 & Passa & Passa & $\begin{array}{l}3.019 \mathrm{~m}^{\prime} \\
\eta=21.7\end{array}$ & $\begin{array}{l}\prime 0.915 \mathrm{~m}^{\prime} \\
\eta=38.9\end{array}$ & N.P. (1) & N.P. ${ }^{(1)}$ & N.P. (1) & N.P. (2) & N.P. ${ }^{(1)}$ & N.P. ${ }^{(1)}$ & N.P. ${ }^{(1)}$ & N.P. ${ }^{(1)}$ & N.P. ${ }^{(1)}$ & N.P..$^{(1)}$ & N.P. ${ }^{(1)}$ & N.P. (3) & $\begin{array}{c}\text { PASSA } \\
\eta=38.9\end{array}$ \\
\hline a: V 12 - P9 & Passa & Passa & $\begin{array}{c}10.183 \mathrm{~m}^{\prime} \\
\eta=5.8\end{array}$ & $\begin{array}{l}\prime 1.217 \mathrm{~m}^{\prime} \\
\eta=11.3\end{array}$ & N.P. ${ }^{(1)}$ & N.P. ${ }^{(1)}$ & N.P. ${ }^{(1)}$ & N.P. (2) & N.P. ${ }^{(1)}$ & N.P. ${ }^{(1)}$ & N.P. ${ }^{(1)}$ & N.P. ${ }^{(1)}$ & N.P. ${ }^{(1)}$ & N.P. ${ }^{(1)}$ & N.P. ${ }^{(1)}$ & N.P. ${ }^{(3)}$ & $\begin{array}{c}\text { PASSA } \\
\eta=11.3\end{array}$ \\
\hline a: V 12 - V 14 & Passa & Passa & $\begin{array}{l}\prime 0.167 \mathrm{~m}^{\prime} \\
\eta=16.5\end{array}$ & $\begin{array}{l}1.094 \mathrm{~m}^{\prime} \\
\eta=44.7\end{array}$ & N.P. ${ }^{(1)}$ & N.P. ${ }^{(1)}$ & N.P. ${ }^{(1)}$ & N.P. (2) & N.P. ${ }^{(1)}$ & N.P. ${ }^{(1)}$ & N.P. ${ }^{(1)}$ & N.P. ${ }^{(1)}$ & N.P. ${ }^{(1)}$ & N.P. ${ }^{(1)}$ & N.P. ${ }^{(1)}$ & N.P. ${ }^{(3)}$ & $\begin{array}{c}\text { PASSA } \\
\eta=44.7\end{array}$ \\
\hline a: V $6-\vee 4$ & Passa & Passa & $\begin{array}{l}\prime 0.207 \mathrm{~m}^{\prime} \\
\eta=13.8\end{array}$ & $\begin{array}{l}\prime 0.938 \mathrm{~m}^{\prime} \\
\eta=34.1\end{array}$ & N.P. ${ }^{(1)}$ & N.P. ${ }^{(1)}$ & N.P. ${ }^{(1)}$ & N.P. (2) & N.P. ${ }^{(1)}$ & N.P. ${ }^{(1)}$ & N.P. ${ }^{(1)}$ & N.P. ${ }^{(1)}$ & N.P. ${ }^{(1)}$ & N.P. ${ }^{(1)}$ & N.P. ${ }^{(1)}$ & N.P. ${ }^{(3)}$ & $\begin{array}{c}\text { PASSA } \\
\eta=34.1\end{array}$ \\
\hline a: P4 - V 1 & Passa & Passa & $\begin{array}{l}\prime 0.207 \mathrm{~m}^{\prime} \\
\eta=10.6 \\
\end{array}$ & $\begin{array}{c}\text { 'P4' } \\
\eta=19.4\end{array}$ & N.P. ${ }^{(1)}$ & N.P. ${ }^{(1)}$ & N.P. (1) & N.P. (2) & N.P. ${ }^{(1)}$ & N.P. ${ }^{(1)}$ & N.P. ${ }^{(1)}$ & N.P. ${ }^{(1)}$ & N.P. ${ }^{(1)}$ & N.P. ${ }^{(1)}$ & N.P. ${ }^{(1)}$ & N.P. ${ }^{(3)}$ & $\begin{array}{c}\text { PASSA } \\
\eta=19.4\end{array}$ \\
\hline a: V 8 - V 7 & Passa & Passa & $\begin{array}{c}10.207 \mathrm{~m}^{\prime} \\
\eta=8.1\end{array}$ & $\begin{array}{l}\prime 0.525 \mathrm{~m}^{\prime} \\
\eta=15.2 \\
\end{array}$ & N.P. ${ }^{(1)}$ & N.P. ${ }^{(1)}$ & N.P. (1) & N.P. (2) & N.P. ${ }^{(1)}$ & N.P. ${ }^{(1)}$ & N.P. ${ }^{(1)}$ & N.P. ${ }^{(1)}$ & N.P. ${ }^{(1)}$ & N.P..$^{(1)}$ & N.P. ${ }^{(1)}$ & N.P. ${ }^{(3)}$ & $\begin{array}{c}\text { PASSA } \\
\eta=15.2\end{array}$ \\
\hline a: V 8 - P13 & Passa & Passa & $\begin{array}{l}1.394 \mathrm{~m}^{\prime} \\
\eta=16.1\end{array}$ & $\begin{array}{l}1.321 \mathrm{~m}^{\prime} \\
\eta=39.8\end{array}$ & N.P. ${ }^{(1)}$ & N.P. ${ }^{(1)}$ & N.P. ${ }^{(1)}$ & N.P. ${ }^{(2)}$ & N.P. ${ }^{(1)}$ & N.P. ${ }^{(1)}$ & N.P. ${ }^{(1)}$ & N.P. ${ }^{(1)}$ & N.P. ${ }^{(1)}$ & N.P. ${ }^{(1)}$ & N.P. ${ }^{(1)}$ & N.P. ${ }^{(3)}$ & $\begin{array}{c}\text { PASSA } \\
\eta=\mathbf{3 9 . 8}\end{array}$ \\
\hline c: P6 - P3 & Passa & Passa & $\begin{array}{c}0.207 \mathrm{~m}^{\prime} \\
\eta=5.9\end{array}$ & $\begin{array}{c}\text { 'P6' } \\
\eta=23.1\end{array}$ & N.P. ${ }^{(1)}$ & N.P. ${ }^{(1)}$ & N.P. ${ }^{(1)}$ & N.P. (2) & N.P. ${ }^{(1)}$ & N.P. ${ }^{(1)}$ & N.P. ${ }^{(1)}$ & N.P. ${ }^{(1)}$ & N.P. ${ }^{(1)}$ & N.P. ${ }^{(1)}$ & N.P. ${ }^{(1)}$ & N.P. ${ }^{(3)}$ & $\begin{array}{c}\text { PASSA } \\
\eta=23.1\end{array}$ \\
\hline
\end{tabular}

\section{Notação:}

Disp.: Disposições relativas às armaduras

Arm.: Armadura mínima e máxima

Q: Estado limite de ruptura relativo ao esforço cortante (combinações não sísmicas)

N,M: Estado limite de ruptura frente a solicitações normais (combinações não sísmicas)

$T_{c}$ : Estado limite de ruptura por torção. Compressão oblíqua.

$T_{\text {st: }}$ Estado limite de ruptura por torção. Tração na alma.

$T_{\text {sl: }}$ Estado limite de ruptura por torção. Tração nas armaduras longitudinais.

TNMx: Estado limite de ruptura por torção. Interação entre torção e esforços normais. Flexão em torno do eixo $X$.

$T V_{x}$ : Estado limite de ruptura por torção. Interação entre torção e esforço cortante no eixo $X$. Compressão oblíqua

$T V_{y}$ : Estado limite de ruptura por torção. Interação entre torção e esforço cortante no eixo Y. Compressão oblíqua

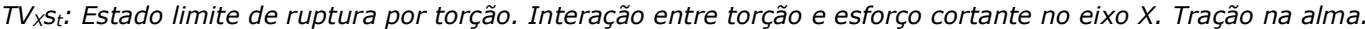

TVYSt: Estado limite de ruptura por torção. Interação entre torção e esforço cortante no eixo Y. Tração na alma.

T,Disp.sl: Estado limite de ruptura por torção. Espaçamento entre as barras da armadura longitudinal.

T,Geom. st: Estado limite de ruptura por torção. Diâmetro mínimo da armadura transversal.

T,Arm.st: Estado limite de ruptura por torção. Quantidade mínima de estribos fechados.

$x$ : Distância à origem da barra

$\eta$ : Coeficiente de aproveitamento (\%)

N.P.: Não procede

$-:-$

Verificações desnecessárias para o tipo de perfil (N.P.)

(1) A verificação do estado limite de ruptura por torção não é necessária, já que não há momento de torção.

(2) A verificação não é necessária, já que não há interação entre torção e esforços normais.

(3) Não há esforços que produzam tensões normais para nenhuma combinação. Portanto, a verificação não é necessária.

\begin{tabular}{|c|c|c|c|c|c|c|}
\hline \multirow{2}{*}{ Vigas } & \multicolumn{5}{|c|}{ VERIFICAÇÕES DE FISSURAÇÃO (ABNT NBR 6118:2014) } & \multirow{2}{*}{ Estado } \\
\hline & $W_{k, F, \text { sup. }}$ & $\mathrm{W}_{\mathrm{k}, \mathrm{F}, \text { Lat.Dir. }}$ & $\mathrm{W}_{\mathrm{k}, \mathrm{F}, \text { inf. }}$ & $\mathrm{W}_{\mathrm{k}, \mathrm{F}, \text { Lat.Esq. }}$ & $\sigma_{\mathrm{s}}$ & \\
\hline a: P1 - V 11 & $\begin{array}{c}\text { x: } 0 \mathrm{~m} \\
\text { Passa }\end{array}$ & N.P.(1) & $\begin{array}{c}\mathrm{x}: 2.31 \mathrm{~m} \\
\text { Passa }\end{array}$ & N.P.(1) & N.P. (2) & PASSA \\
\hline b: V 11 - P2 & 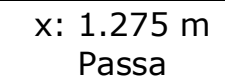 & N.P.(1) & $\begin{array}{l}\text { x: } 0 \mathrm{~m} \\
\text { Passa }\end{array}$ & N.P.(1) & N.P. (2) & PASSA \\
\hline c: P2 - P3 & $\begin{array}{l}\text { x: } 0 \mathrm{~m} \\
\text { Passa }\end{array}$ & N.P. $(1)$ & $\begin{array}{c}\mathrm{x}: 2.457 \mathrm{~m} \\
\text { Passa }\end{array}$ & N.P. $(1)$ & N.P. ${ }^{(2)}$ & PASSA \\
\hline $\mathrm{a}: \mathrm{V} 9-\mathrm{P} 4$ & 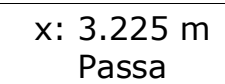 & N.P. (1) & 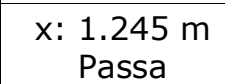 & N.P. ${ }^{(1)}$ & N.P. ${ }^{(2)}$ & PASSA \\
\hline
\end{tabular}




\section{Pontifícia Universidade Católlca}

DO RIO DE JANEIRO

\begin{tabular}{|c|c|c|c|c|c|c|}
\hline \multirow{2}{*}{ Vigas } & \multicolumn{5}{|c|}{ VERIFICAÇÕES DE FISSURAÇÃO (ABNT NBR 6118:2014) } & \multirow{2}{*}{ Estado } \\
\hline & $W_{k, F, \text { sup. }}$ & $\mathrm{W}_{\mathrm{k}, \mathrm{F}, \text { Lat.Dir. }}$ & $W_{k, F, i n f .}$ & $W_{k, F, \text { Lat.Esq. }}$ & $\sigma_{\mathrm{s}}$ & \\
\hline a: P5 - P6 & $\begin{array}{c}\text { x: } 0 \mathrm{~m} \\
\text { Passa }\end{array}$ & N.P. ${ }^{(1)}$ & $\begin{array}{c}\mathrm{x}: 2.12 \mathrm{~m} \\
\text { Passa }\end{array}$ & N.P. ${ }^{(1)}$ & N.P. ${ }^{(2)}$ & PASSA \\
\hline $\mathrm{a}: \mathrm{P7}-\mathrm{P} 8$ & $\begin{array}{c}\mathrm{x}: 4.25 \mathrm{~m} \\
\text { Passa }\end{array}$ & N.P. ${ }^{(1)}$ & $\begin{array}{c}\mathrm{x}: 2.31 \mathrm{~m} \\
\text { Passa }\end{array}$ & N.P. ${ }^{(1)}$ & N.P. ${ }^{(2)}$ & PASSA \\
\hline $\mathrm{a}: \mathrm{V} 12$ - P9 & $\begin{array}{c}\mathrm{x}: 1.475 \mathrm{~m} \\
\text { Passa }\end{array}$ & N.P. ${ }^{(1)}$ & $\begin{array}{c}\text { x: } 0.7 \mathrm{~m} \\
\text { Passa }\end{array}$ & N.P. ${ }^{(1)}$ & N.P. ${ }^{(2)}$ & PASSA \\
\hline a: P10 - P11 & $\begin{array}{c}\mathrm{x}: 4.25 \mathrm{~m} \\
\text { Passa }\end{array}$ & N.P. ${ }^{(1)}$ & $\begin{array}{l}\mathrm{x}: 2.31 \mathrm{~m} \\
\text { Passa }\end{array}$ & N.P. ${ }^{(1)}$ & N.P. ${ }^{(2)}$ & PASSA \\
\hline b: P11 - P12 & $\begin{array}{l}\mathrm{x}: 0 \mathrm{~m} \\
\text { Passa }\end{array}$ & N.P. ${ }^{(1)}$ & $\begin{array}{c}\mathrm{x}: 2.35 \mathrm{~m} \\
\text { Passa }\end{array}$ & N.P. ${ }^{(1)}$ & N.P. ${ }^{(2)}$ & PASSA \\
\hline $\mathrm{a}: \mathrm{V} 12-\mathrm{V} 14$ & N.P. ${ }^{(1)}$ & N.P. ${ }^{(1)}$ & $\begin{array}{l}\mathrm{x}: 1.3 \mathrm{~m} \\
\text { Passa }\end{array}$ & N.P. ${ }^{(1)}$ & N.P. ${ }^{(2)}$ & PASSA \\
\hline b: V $14-P 13$ & $\begin{array}{c}\mathrm{x}: 1.275 \mathrm{~m} \\
\text { Passa }\end{array}$ & N.P. ${ }^{(1)}$ & $\begin{array}{l}\text { x: } 0 \text { m } \\
\text { Passa }\end{array}$ & N.P. ${ }^{(1)}$ & N.P. ${ }^{(2)}$ & PASSA \\
\hline a: P14 - P15 & $\begin{array}{c}\mathrm{x}: 4.225 \mathrm{~m} \\
\text { Passa }\end{array}$ & N.P. ${ }^{(1)}$ & $\begin{array}{c}\mathrm{x}: 2.113 \mathrm{~m} \\
\text { Passa }\end{array}$ & N.P. ${ }^{(1)}$ & N.P. ${ }^{(2)}$ & PASSA \\
\hline b: P15 - V 14 & $\begin{array}{c}\text { x: } 0 \mathrm{~m} \\
\text { Passa }\end{array}$ & N.P. ${ }^{(1)}$ & $\begin{array}{c}\mathrm{x}: 1.25 \mathrm{~m} \\
\text { Passa }\end{array}$ & N.P. ${ }^{(1)}$ & $\begin{array}{l}\text { x: } 0 \text { m } \\
\text { Passa }\end{array}$ & PASSA \\
\hline c: V $14-$ V 16 & N.P. ${ }^{(1)}$ & N.P. ${ }^{(1)}$ & $\begin{array}{c}\mathrm{x}: 0.892 \mathrm{~m} \\
\text { Passa }\end{array}$ & N.P. ${ }^{(1)}$ & N.P. ${ }^{(2)}$ & PASSA \\
\hline $\mathrm{d}: \mathrm{V} 16-\mathrm{P} 16$ & $\begin{array}{c}\mathrm{x}: 1.825 \mathrm{~m} \\
\text { Passa }\end{array}$ & N.P. ${ }^{(1)}$ & $\begin{array}{l}\text { x: } 0 \mathrm{~m} \\
\text { Passa }\end{array}$ & N.P. ${ }^{(1)}$ & N.P. ${ }^{(2)}$ & PASSA \\
\hline a: P14 - P10 & $\begin{array}{c}\mathrm{x}: 4.25 \mathrm{~m} \\
\text { Passa }\end{array}$ & N.P. ${ }^{(1)}$ & $\begin{array}{c}\mathrm{x}: 2.125 \mathrm{~m} \\
\text { Passa }\end{array}$ & N.P. ${ }^{(1)}$ & N.P. ${ }^{(2)}$ & PASSA \\
\hline b: P10 - P7 & $\begin{array}{c}\mathrm{x}: 2.475 \mathrm{~m} \\
\text { Passa }\end{array}$ & N.P. ${ }^{(1)}$ & $\begin{array}{l}\mathrm{x}: 1.238 \mathrm{~m} \\
\text { Passa }\end{array}$ & N.P. ${ }^{(1)}$ & N.P. ${ }^{(2)}$ & PASSA \\
\hline c: P7 - P1 & $\begin{array}{c}\mathrm{x}: 3.975 \mathrm{~m} \\
\text { Passa }\end{array}$ & N.P. ${ }^{(1)}$ & $\begin{array}{c}\mathrm{x}: 2.363 \mathrm{~m} \\
\text { Passa }\end{array}$ & N.P. ${ }^{(1)}$ & N.P. ${ }^{(2)}$ & PASSA \\
\hline a: V $6-$ V 4 & N.P. ${ }^{(1)}$ & N.P. ${ }^{(1)}$ & $\begin{array}{l}\mathrm{x}: 1.275 \mathrm{~m} \\
\text { Passa }\end{array}$ & N.P. ${ }^{(1)}$ & N.P. ${ }^{(2)}$ & PASSA \\
\hline $\mathrm{a}: \mathrm{P} 15-\mathrm{V} 7$ & $\begin{array}{l}\text { x: } 0 \text { m } \\
\text { Passa }\end{array}$ & N.P. ${ }^{(1)}$ & $\begin{array}{c}\mathrm{x}: 1.6 \mathrm{~m} \\
\text { Passa }\end{array}$ & N.P. ${ }^{(1)}$ & N.P. ${ }^{(2)}$ & PASSA \\
\hline $\mathrm{b}: \mathrm{V} 7-\mathrm{P} 11$ & $\begin{array}{c}\mathrm{x}: 2.5 \mathrm{~m} \\
\text { Passa }\end{array}$ & N.P. ${ }^{(1)}$ & $\begin{array}{c}\text { x: } 0 \mathrm{~m} \\
\text { Passa }\end{array}$ & N.P. ${ }^{(1)}$ & N.P. ${ }^{(2)}$ & PASSA \\
\hline C: P11 - P8 & $\begin{array}{c}\mathrm{x}: 0 \mathrm{~m} \\
\text { Passa }\end{array}$ & N.P. ${ }^{(1)}$ & $\begin{array}{c}\mathrm{x}: 1.183 \mathrm{~m} \\
\text { Passa }\end{array}$ & N.P. ${ }^{(1)}$ & N.P. ${ }^{(2)}$ & PASSA \\
\hline d: P8 - P5 & $\begin{array}{l}\mathrm{x}: 1.025 \mathrm{~m} \\
\text { Passa }\end{array}$ & N.P. ${ }^{(1)}$ & N.P. ${ }^{(1)}$ & N.P. ${ }^{(1)}$ & N.P. ${ }^{(2)}$ & PASSA \\
\hline a: P5 - P2 & $\begin{array}{l}\text { x: } 0 \text { m } \\
\text { Passa }\end{array}$ & N.P. ${ }^{(1)}$ & $\begin{array}{c}\mathrm{x}: 1.4 \mathrm{~m} \\
\text { Passa }\end{array}$ & N.P. ${ }^{(1)}$ & N.P. ${ }^{(2)}$ & PASSA \\
\hline $\mathrm{a}: \mathrm{V} 8-\mathrm{V} 7$ & N.P. ${ }^{(1)}$ & N.P. ${ }^{(1)}$ & $\begin{array}{c}\mathrm{x}: 0.825 \mathrm{~m} \\
\text { Passa }\end{array}$ & N.P. ${ }^{(1)}$ & N.P. ${ }^{(2)}$ & PASSA \\
\hline a: $P 9-V 3$ & $\begin{array}{l}\text { x: } 0 \mathrm{~m} \\
\text { Passa }\end{array}$ & N.P. ${ }^{(1)}$ & $\begin{array}{l}\mathrm{x}: 1.704 \mathrm{~m} \\
\text { Passa }\end{array}$ & N.P. ${ }^{(1)}$ & N.P. ${ }^{(2)}$ & PASSA \\
\hline a: P16 - P12 & $\begin{array}{c}\mathrm{x}: 4.25 \mathrm{~m} \\
\text { Passa }\end{array}$ & N.P. ${ }^{(1)}$ & $\begin{array}{c}\mathrm{x}: 2.125 \mathrm{~m} \\
\text { Passa }\end{array}$ & N.P. ${ }^{(1)}$ & N.P. ${ }^{(2)}$ & PASSA \\
\hline b: P12 - P6 & $\begin{array}{l}\text { x: } 0 \mathrm{~m} \\
\text { Passa }\end{array}$ & N.P. ${ }^{(1)}$ & $\begin{array}{c}\mathrm{x}: 1.825 \mathrm{~m} \\
\text { Passa }\end{array}$ & N.P. ${ }^{(1)}$ & N.P. ${ }^{(2)}$ & PASSA \\
\hline
\end{tabular}




\begin{tabular}{|c|c|c|c|c|c|c|c|}
\hline \multirow{2}{*}{ Vigas } & \multicolumn{6}{|c|}{ VERIFICAÇÕES DE FISSURAÇÃO (ABNT NBR 6118:2014) } & \multirow{2}{*}{ Estado } \\
\hline & $\mathrm{W}_{\mathrm{k}, \mathrm{F}, \text { sup. }}$ & $\mathrm{W}_{\mathrm{k}, \mathrm{F}, \text { Lat.Dir. }}$ & $W_{k, F, \text { inf. }}$ & $\mathrm{W}_{\mathrm{k}, \mathrm{F}, \text { Lat.Esq. }}$ & $\sigma_{\mathrm{s}}$ & - & \\
\hline a: P4 - V 1 & $\begin{array}{c}\mathrm{x}: 0 \mathrm{~m} \\
\text { Passa }\end{array}$ & N.P. (1) & N.P. ${ }^{(1)}$ & N.P. ${ }^{(1)}$ & N.P. (2) & N.P. (3) & PASSA \\
\hline a: V $8-P 13$ & $\begin{array}{c}\mathrm{x}: 1.6 \mathrm{~m} \\
\text { Passa }\end{array}$ & N.P. ${ }^{(1)}$ & N.P. ${ }^{(1)}$ & N.P. ${ }^{(1)}$ & N.P. (2) & N.P. (3) & PASSA \\
\hline c: P6 - P3 & $\begin{array}{c}\text { x: } 0 \text { m } \\
\text { Passa }\end{array}$ & N.P. $(1)$ & N.P. ${ }^{(1)}$ & N.P. $(1)$ & N.P. (2) & N.P. (3) & PASSA \\
\hline
\end{tabular}

\footnotetext{
Notação:

$W_{k, F, \text { sup.: }}$ Controle da fissuração através da limitação da abertura estimada das fissuras: Face superior $W_{k, F, L a t . D i r .:}$ Controle da fissuração através da limitação da abertura estimada das fissuras: Face lateral direita $W_{k, F, \text { inf. }}$ : Controle da fissuração através da limitação da abertura estimada das fissuras: Face inferior

$W_{k, F, \text { Lat. Esq. }}$ Controle da fissuração através da limitação da abertura estimada das fissuras: Face lateral esquerda $\sigma_{s}:$ Armaduras longitudinais mínimas

x: Distância à origem da barra

$\eta$ : Coeficiente de aproveitamento (\%)

N.P.: Não procede

$-:$ -

Verificações desnecessárias para o tipo de perfil (N.P.):

(1) A verificação não é necessária, já que não há nenhuma armadura tracionada.

(2) A verificação não é necessária, já que a tensão de tração máxima no concreto não supera a resistência à tração do mesmo.

(3) Não há esforços que produzam tensões normais para nenhuma combinação. Portanto, a verificação não é necessária.
}

\begin{tabular}{|c|c|c|c|c|}
\hline \multicolumn{5}{|c|}{ Verificações de flecha } \\
\hline Vigas & $\begin{array}{c}\text { Sobrecarga } \\
\text { (Característica) } \\
\mathrm{f}_{\mathrm{i}, \mathrm{Q}} \leq \mathrm{f}_{\mathrm{i}, \mathrm{Q}, \lim } \\
\mathrm{f}_{\mathrm{i}, \mathrm{Q}, \lim }=\mathrm{L} / 350\end{array}$ & $\begin{array}{c}\text { No tempo infinito } \\
\text { (Quase permanente) } \\
\mathrm{f}_{\mathrm{T}, \max } \leq \mathrm{f}_{\mathrm{T}, \lim } \\
\mathrm{f}_{\mathrm{T}, \lim }=\mathrm{L} / 250\end{array}$ & $\begin{array}{c}\text { Ativa } \\
\text { (Característica) } \\
\mathrm{f}_{\mathrm{A}, \max } \leq \mathrm{f}_{\mathrm{A}, \lim } \\
\mathrm{f}_{\mathrm{A}, \lim }=\text { Mín. }(10.00, \mathrm{~L} / 500)\end{array}$ & Estado \\
\hline a: P1 - V 11 & $\begin{array}{l}f_{i, Q}: 0.00 \mathrm{~mm} \\
f_{i, Q}, \lim : 13.29 \mathrm{~mm}\end{array}$ & $\begin{array}{l}\mathrm{f}_{\mathrm{T}, \max }: 0.43 \mathrm{~mm} \\
\mathrm{f}_{\mathrm{T}, \mathrm{lim}}: 18.60 \mathrm{~mm}\end{array}$ & $\begin{array}{l}\mathrm{f}_{\mathrm{A}, \max }: 0.34 \mathrm{~mm} \\
\mathrm{f}_{\mathrm{A}, \lim }: 9.30 \mathrm{~mm}\end{array}$ & PASSA \\
\hline b: V $11-P 2$ & $\begin{array}{l}\mathrm{f}_{\mathrm{i}, \mathrm{Q}}: 0.00 \mathrm{~mm} \\
\mathrm{f}_{\mathrm{i}, \mathrm{Q}, \lim }: 13.29 \mathrm{~mm}\end{array}$ & $\begin{array}{l}\mathrm{f}_{\mathrm{T}, \max }: 0.29 \mathrm{~mm} \\
\mathrm{f}_{\mathrm{T}, \mathrm{lim}}: 18.60 \mathrm{~mm}\end{array}$ & $\begin{array}{l}\mathrm{f}_{\mathrm{A}, \max }: 0.23 \mathrm{~mm} \\
\mathrm{f}_{\mathrm{A}, \lim }: 9.30 \mathrm{~mm}\end{array}$ & PASSA \\
\hline c: P2 - P3 & $\begin{array}{l}f_{i, Q}: 0.00 \mathrm{~mm} \\
f_{i, Q}, \lim : 12.29 \mathrm{~mm}\end{array}$ & $\begin{array}{l}\mathrm{f}_{\mathrm{T}, \max }: 0.06 \mathrm{~mm} \\
\mathrm{f}_{\mathrm{T}, \mathrm{lim}}: 15.51 \mathrm{~mm}\end{array}$ & $\begin{array}{l}\mathrm{f}_{\mathrm{A}, \max }: 0.02 \mathrm{~mm} \\
\mathrm{f}_{\mathrm{A}, \lim }: 1.23 \mathrm{~mm}\end{array}$ & PASSA \\
\hline $\mathrm{a}: \mathrm{V} 9-\mathrm{P} 4$ & $\begin{array}{l}f_{i, Q}: 0.00 \mathrm{~mm} \\
f_{i, Q}, \lim : 9.21 \mathrm{~mm}\end{array}$ & $\begin{array}{l}\mathrm{f}_{\mathrm{T}, \max }: 0.40 \mathrm{~mm} \\
\mathrm{f}_{\mathrm{T}, \mathrm{lim}}: 12.90 \mathrm{~mm}\end{array}$ & $\begin{array}{l}f_{A, \max }: 0.34 \mathrm{~mm} \\
f_{A, \lim }: 6.45 \mathrm{~mm}\end{array}$ & PASSA \\
\hline a: P5 - P6 & $\begin{array}{l}f_{i, Q}: 0.00 \mathrm{~mm} \\
f_{i, Q}, \lim : 12.36 \mathrm{~mm}\end{array}$ & $\begin{array}{l}\mathrm{f}_{\mathrm{T}, \max }: 0.71 \mathrm{~mm} \\
\mathrm{f}_{\mathrm{T}, \mathrm{lim}}: 17.30 \mathrm{~mm}\end{array}$ & $\begin{array}{l}\mathrm{f}_{\mathrm{A}, \max }: 0.60 \mathrm{~mm} \\
\mathrm{f}_{\mathrm{A}, \lim }: 8.65 \mathrm{~mm}\end{array}$ & PASSA \\
\hline a: P7 - P8 & $\begin{array}{l}f_{i, Q}: 0.01 \mathrm{~mm} \\
f_{i, Q}, \lim : 12.14 \mathrm{~mm}\end{array}$ & $\begin{array}{l}\mathrm{f}_{\mathrm{T}, \max }: 0.74 \mathrm{~mm} \\
\mathrm{f}_{\mathrm{T}, \mathrm{lim}}: 17.00 \mathrm{~mm}\end{array}$ & $\begin{array}{l}\mathrm{f}_{\mathrm{A}, \max }: 0.63 \mathrm{~mm} \\
\mathrm{f}_{\mathrm{A}, \lim }: 8.50 \mathrm{~mm}\end{array}$ & PASSA \\
\hline $\mathrm{a}: \mathrm{V} 12$ - P9 & $\begin{array}{l}f_{i, Q}: 0.00 \mathrm{~mm} \\
f_{i, Q, \lim }: 4.21 \mathrm{~mm}\end{array}$ & $\begin{array}{l}\mathrm{f}_{\mathrm{T}, \max }: 0.03 \mathrm{~mm} \\
\mathrm{f}_{\mathrm{T}, \mathrm{lim}}: 5.90 \mathrm{~mm}\end{array}$ & $\begin{array}{l}\mathrm{f}_{\mathrm{A}, \max }: 0.02 \mathrm{~mm} \\
\mathrm{f}_{\mathrm{A}, \lim }: 2.95 \mathrm{~mm}\end{array}$ & PASSA \\
\hline a: P10 - P11 & $\begin{array}{l}\mathrm{f}_{\mathrm{i}, \mathrm{Q}}: 0.00 \mathrm{~mm} \\
\mathrm{f}_{\mathrm{i}, \mathrm{Q}, \lim }: 12.14 \mathrm{~mm}\end{array}$ & $\begin{array}{l}\mathrm{f}_{\mathrm{T}, \max }: 0.64 \mathrm{~mm} \\
\mathrm{f}_{\mathrm{T}, \mathrm{lim}}: 17.00 \mathrm{~mm}\end{array}$ & $\begin{array}{l}\mathrm{f}_{\mathrm{A}, \max }: 0.54 \mathrm{~mm} \\
\mathrm{f}_{\mathrm{A}, \lim }: 8.50 \mathrm{~mm}\end{array}$ & PASSA \\
\hline $\mathrm{b}: \mathrm{P} 11-\mathrm{P} 12$ & $\begin{array}{l}f_{i, Q}: 0.00 \mathrm{~mm} \\
f_{i, Q, \lim }: 13.43 \mathrm{~mm}\end{array}$ & $\begin{array}{l}\mathrm{f}_{\mathrm{T}, \max }: 0.67 \mathrm{~mm} \\
\mathrm{f}_{\mathrm{T}, \mathrm{lim}}: 18.80 \mathrm{~mm}\end{array}$ & $\begin{array}{l}\mathrm{f}_{\mathrm{A}, \max }: 0.56 \mathrm{~mm} \\
\mathrm{f}_{\mathrm{A}, \lim }: 9.40 \mathrm{~mm}\end{array}$ & PASSA \\
\hline a: V $12-$ V 14 & $\begin{array}{l}\mathrm{f}_{\mathrm{i}, \mathrm{Q}}: 0.00 \mathrm{~mm} \\
\mathrm{f}_{\mathrm{i}, \mathrm{Q}, \lim }: 7.79 \mathrm{~mm}\end{array}$ & $\begin{array}{l}\mathrm{f}_{\mathrm{T}, \max }: 0.31 \mathrm{~mm} \\
\mathrm{f}_{\mathrm{T}, \mathrm{lim}}: 10.90 \mathrm{~mm}\end{array}$ & $\begin{array}{l}\mathrm{f}_{\mathrm{A}, \max }: 0.26 \mathrm{~mm} \\
\mathrm{f}_{\mathrm{A}, \mathrm{lim}}: 5.45 \mathrm{~mm}\end{array}$ & PASSA \\
\hline $\mathrm{b}: \mathrm{V} 14-\mathrm{P} 13$ & $\begin{array}{l}f_{i, Q}: 0.00 \mathrm{~mm} \\
f_{i, Q}, \lim : 7.79 \mathrm{~mm}\end{array}$ & $\begin{array}{l}\mathrm{f}_{\mathrm{T}, \max }: 0.30 \mathrm{~mm} \\
\mathrm{f}_{\mathrm{T}, \mathrm{lim}}: 10.90 \mathrm{~mm}\end{array}$ & $\begin{array}{l}\mathrm{f}_{\mathrm{A}, \max }: 0.25 \mathrm{~mm} \\
\mathrm{f}_{\mathrm{A}, \lim }: 5.45 \mathrm{~mm}\end{array}$ & PASSA \\
\hline a: P14 - P15 & $\begin{array}{l}\mathrm{f}_{\mathrm{i}, \mathrm{Q}}: 0.00 \mathrm{~mm} \\
\mathrm{f}_{\mathrm{i}, \mathrm{Q}, \lim : 12.07 \mathrm{~mm}}\end{array}$ & $\begin{array}{l}\mathrm{f}_{\mathrm{T}, \max }: 0.43 \mathrm{~mm} \\
\mathrm{f}_{\mathrm{T}, \lim }: 16.90 \mathrm{~mm}\end{array}$ & $\begin{array}{l}\mathrm{f}_{\mathrm{A}, \max }: 0.36 \mathrm{~mm} \\
\mathrm{f}_{\mathrm{A}, \mathrm{lim}:}: 8.45 \mathrm{~mm}\end{array}$ & PASSA \\
\hline
\end{tabular}




\section{Pontifícia Universidade Católlca}

\begin{tabular}{|c|c|c|c|c|}
\hline \multicolumn{5}{|c|}{ Verificações de flecha } \\
\hline Vigas & $\begin{array}{c}\text { Sobrecarga } \\
\text { (Característica) } \\
\mathrm{f}_{\mathrm{i}, \mathrm{Q}} \leq \mathrm{f}_{\mathrm{i}, \mathrm{Q}}, \lim \\
\mathrm{f}_{\mathrm{i}, \mathrm{Q}} \lim =\mathrm{L} / 350 \\
\end{array}$ & $\begin{array}{c}\text { No tempo infinito } \\
\text { (Quase permanente) } \\
f_{\mathrm{T}, \max } \leq \mathrm{f}_{\mathrm{T}, \lim } \\
\mathrm{f}_{\mathrm{T}, \lim }=\mathrm{L} / 250\end{array}$ & $\begin{array}{c}\text { Ativa } \\
\text { (Característica) } \\
\mathrm{f}_{\mathrm{A}, \max } \leq \mathrm{f}_{\mathrm{A}, \lim } \\
\mathrm{f}_{\mathrm{A}, \mathrm{lim}}=\text { Mín. }(10.00, \mathrm{~L} / 500)\end{array}$ & Estado \\
\hline b: P15 - V 14 & $\begin{array}{l}f_{i, Q}: 0.00 \mathrm{~mm} \\
f_{i, Q, \lim }: 13.36 \mathrm{~mm}\end{array}$ & $\begin{array}{l}\mathrm{f}_{\mathrm{T}, \max }: 0.66 \mathrm{~mm} \\
\mathrm{f}_{\mathrm{T}, \lim : 18.70 \mathrm{~mm}}\end{array}$ & $\begin{array}{l}f_{A, \max }: 0.56 \mathrm{~mm} \\
f_{A, l i m}: 9.35 \mathrm{~mm}\end{array}$ & PASSA \\
\hline c: V $14-\mathrm{V} 16$ & $\begin{array}{l}f_{\mathrm{i}, \mathrm{Q}}: 0.00 \mathrm{~mm} \\
\mathrm{f}_{\mathrm{i}, \mathrm{Q}, \lim }: 13.36 \mathrm{~mm}\end{array}$ & $\begin{array}{l}f_{T, \max }: 1.03 \mathrm{~mm} \\
f_{\mathrm{T}, \mathrm{lim}}: 18.70 \mathrm{~mm}\end{array}$ & $\begin{array}{l}f_{A, \max }: 0.87 \mathrm{~mm} \\
f_{\mathrm{A}, \lim }: 9.35 \mathrm{~mm}\end{array}$ & PASSA \\
\hline $\mathrm{d}: \mathrm{V} 16-\mathrm{P} 16$ & $\begin{array}{l}f_{i, Q}: 0.00 \mathrm{~mm} \\
f_{i, Q}, \lim : 13.36 \mathrm{~mm}\end{array}$ & $\begin{array}{l}f_{T, \max }: 0.95 \mathrm{~mm} \\
f_{\mathrm{T}, \lim : 18.70 \mathrm{~mm}}\end{array}$ & $\begin{array}{l}\mathrm{f}_{\mathrm{A}, \max }: 0.81 \mathrm{~mm} \\
\mathrm{f}_{\mathrm{A}, \lim :}: 9.35 \mathrm{~mm}\end{array}$ & PASSA \\
\hline a: P14 - P10 & $\begin{array}{l}f_{\mathrm{f}, \mathrm{Q}}: 0.00 \mathrm{~mm} \\
\mathrm{f}_{\mathrm{i}, \mathrm{Q}, \lim : 12.14 \mathrm{~mm}}\end{array}$ & $\begin{array}{l}f_{T, \max }: 0.55 \mathrm{~mm} \\
f_{T, \lim : 17.00 \mathrm{~mm}}\end{array}$ & $\begin{array}{l}f_{A, \max }: 0.46 \mathrm{~mm} \\
f_{\mathrm{A}, \mathrm{lim}}: 8.50 \mathrm{~mm}\end{array}$ & PASSA \\
\hline b: P10 - P7 & $\begin{array}{l}f_{i, Q}: 0.00 \mathrm{~mm} \\
f_{i, Q}, \lim : 7.07 \mathrm{~mm}\end{array}$ & $\begin{array}{l}f_{T, \text { max }}: 0.02 \mathrm{~mm} \\
f_{\mathrm{T}, \lim :}: 9.90 \mathrm{~mm}\end{array}$ & $\begin{array}{l}\mathrm{f}_{\mathrm{A}, \max }: 0.02 \mathrm{~mm} \\
\mathrm{f}_{\mathrm{A}, \text { lim }}: 4.95 \mathrm{~mm}\end{array}$ & PASSA \\
\hline c: P7 - P1 & $\begin{array}{l}f_{i, Q}: 0.00 \mathrm{~mm} \\
f_{i, Q}, \lim : 11.36 \mathrm{~mm}\end{array}$ & $\begin{array}{l}f_{\mathrm{T}, \max }: 0.59 \mathrm{~mm} \\
\mathrm{f}_{\mathrm{T}, \lim : 15.90 \mathrm{~mm}}\end{array}$ & $\begin{array}{l}f_{A, \max }: 0.49 \mathrm{~mm} \\
f_{\mathrm{A}, \lim }: 7.95 \mathrm{~mm}\end{array}$ & PASSA \\
\hline a: V 6 - V 4 & $\begin{array}{l}f_{i, Q}: 0.00 \mathrm{~mm} \\
f_{i, Q}, \lim : 7.29 \mathrm{~mm}\end{array}$ & $\begin{array}{l}\mathrm{f}_{\mathrm{T}, \max }: 0.28 \mathrm{~mm} \\
\mathrm{f}_{\mathrm{T}, \mathrm{lim}}: 10.20 \mathrm{~mm}\end{array}$ & $\begin{array}{l}\mathrm{f}_{\mathrm{A}, \max }: 0.23 \mathrm{~mm} \\
\mathrm{f}_{\mathrm{A}, \mathrm{lim}}: 5.10 \mathrm{~mm}\end{array}$ & PASSA \\
\hline a: P4 - V 1 & $\begin{array}{l}f_{i, Q}: 0.00 \mathrm{~mm} \\
f_{i, Q}, \lim : 3.29 \mathrm{~mm}\end{array}$ & $\begin{array}{l}f_{T, \text { max }}: 0.00 \mathrm{~mm} \\
f_{\mathrm{T}, \lim :}: 4.60 \mathrm{~mm}\end{array}$ & $\begin{array}{l}f_{A, \max }: 0.00 \mathrm{~mm} \\
f_{\mathrm{A}, \text { limm }}: 2.30 \mathrm{~mm}\end{array}$ & PASSA \\
\hline a: P15 - V 7 & $\begin{array}{l}f_{\mathrm{i}, \mathrm{Q}}: 0.00 \mathrm{~mm} \\
\mathrm{f}_{\mathrm{i}, \mathrm{Q}, \lim : 12.14 \mathrm{~mm}}\end{array}$ & $\begin{array}{l}f_{T, \max }: 0.77 \mathrm{~mm} \\
f_{\mathrm{T}, \lim : 17.00 \mathrm{~mm}}\end{array}$ & $\begin{array}{l}\mathrm{f}_{\mathrm{A}, \max }: 0.64 \mathrm{~mm} \\
\mathrm{f}_{\mathrm{A}, \lim }: 8.50 \mathrm{~mm}\end{array}$ & PASSA \\
\hline $\mathrm{b}: \mathrm{V} 7$ - P11 & $\begin{array}{l}f_{\mathrm{i}, \mathrm{Q}}: 0.00 \mathrm{~mm} \\
\mathrm{f}_{\mathrm{i}, \mathrm{Q}, \lim }: 12.14 \mathrm{~mm}\end{array}$ & $\begin{array}{l}f_{\mathrm{T}_{T, \max }: 0.83 \mathrm{~mm}} \\
\mathrm{f}_{\mathrm{T}, \mathrm{lim}: 17.00 \mathrm{~mm}}\end{array}$ & $\begin{array}{l}f_{A, \max }: 0.69 \mathrm{~mm} \\
f_{A, l i m}: 8.50 \mathrm{~mm}\end{array}$ & PASSA \\
\hline c: P11 - P8 & $\begin{array}{l}f_{i, Q}: 0.00 \mathrm{~mm} \\
f_{i, Q}, \lim : 7.07 \mathrm{~mm}\end{array}$ & $\begin{array}{l}f_{T, \max }: 0.06 \mathrm{~mm} \\
f_{\mathrm{T}, \lim :}: 8.66 \mathrm{~mm}\end{array}$ & $\begin{array}{l}f_{A, \max }: 0.05 \mathrm{~mm} \\
f_{A, l i m}: 4.30 \mathrm{~mm}\end{array}$ & PASSA \\
\hline d: P8 - P5 & $\begin{array}{l}f_{i, Q}: 0.00 \mathrm{~mm} \\
f_{i, Q, \lim }: 2.93 \mathrm{~mm}\end{array}$ & $\begin{array}{l}\mathrm{f}_{\mathrm{T}, \max }: 0.00 \mathrm{~mm} \\
\mathrm{f}_{\mathrm{T}, \mathrm{lim}}: 4.10 \mathrm{~mm}\end{array}$ & $\begin{array}{l}\mathrm{f}_{\mathrm{A}, \max }: 0.00 \mathrm{~mm} \\
\mathrm{f}_{\mathrm{A}, \mathrm{lim}}: 2.05 \mathrm{~mm}\end{array}$ & PASSA \\
\hline a: P5 - P2 & $\begin{array}{l}f_{i, Q}: 0.00 \mathrm{~mm} \\
f_{i, Q, \lim }: 8.00 \mathrm{~mm}\end{array}$ & $\begin{array}{l}f_{T, \max }: 0.11 \mathrm{~mm} \\
\mathrm{f}_{\mathrm{T}, \lim : 11.20 \mathrm{~mm}}\end{array}$ & $\begin{array}{l}f_{A, \max }: 0.09 \mathrm{~mm} \\
f_{\mathrm{A}, \lim }: 5.60 \mathrm{~mm}\end{array}$ & PASSA \\
\hline a: V 8 - V 7 & $\begin{array}{l}\mathrm{f}_{\mathrm{i}, \mathrm{Q}}: 0.00 \mathrm{~mm} \\
\mathrm{f}_{\mathrm{i}, \mathrm{Q}, \lim }: 4.71 \mathrm{~mm}\end{array}$ & $\begin{array}{l}\mathrm{f}_{\mathrm{T}, \max }: 0.06 \mathrm{~mm} \\
\mathrm{f}_{\mathrm{T}, \lim : 6.60 \mathrm{~mm}}\end{array}$ & $\begin{array}{l}f_{A, \max }: 0.05 \mathrm{~mm} \\
f_{A, l i m}: 3.30 \mathrm{~mm}\end{array}$ & PASSA \\
\hline a: $P 9-V 3$ & $\begin{array}{l}f_{i, Q}: 0.00 \mathrm{~mm} \\
f_{i, Q, \lim }: 7.57 \mathrm{~mm}\end{array}$ & $\begin{array}{l}\mathrm{f}_{\mathrm{T}, \max }: 0.18 \mathrm{~mm} \\
\mathrm{f}_{\mathrm{T}, \mathrm{lim}}: 10.60 \mathrm{~mm}\end{array}$ & $\begin{array}{l}\mathrm{f}_{\mathrm{A}, \max }: 0.15 \mathrm{~mm} \\
\mathrm{f}_{\mathrm{A}, \mathrm{lim}}: 5.30 \mathrm{~mm}\end{array}$ & PASSA \\
\hline a: V 8 - P13 & $\begin{array}{l}f_{i, Q}: 0.00 \mathrm{~mm} \\
f_{i, Q, \lim }: 4.57 \mathrm{~mm}\end{array}$ & $\begin{array}{l}f_{\mathrm{T}_{\mathrm{T} \text { max }}: 0.03 \mathrm{~mm}} \\
\mathrm{f}_{\mathrm{T}, \lim :}: 6.40 \mathrm{~mm}\end{array}$ & $\begin{array}{l}f_{A, \max }: 0.02 \mathrm{~mm} \\
f_{A, l i m}: 3.20 \mathrm{~mm}\end{array}$ & PASSA \\
\hline a: P16 - P12 & $\begin{array}{l}f_{\mathrm{i}, \mathrm{Q}}: 0.00 \mathrm{~mm} \\
\mathrm{f}_{\mathrm{i}, \mathrm{Q}, \lim }: 12.14 \mathrm{~mm}\end{array}$ & $\begin{array}{l}f_{\mathrm{T}_{\mathrm{T} \text { max }}: 0.51 \mathrm{~mm}} \\
\mathrm{f}_{\mathrm{T}, \mathrm{lim}}: 17.00 \mathrm{~mm}\end{array}$ & $\begin{array}{l}f_{A, \max }: 0.42 \mathrm{~mm} \\
\mathrm{f}_{\mathrm{A}, \mathrm{lim}}: 8.50 \mathrm{~mm}\end{array}$ & PASSA \\
\hline b: P12 - P6 & $\begin{array}{l}f_{\mathrm{i}, \mathrm{Q}}: 0.00 \mathrm{~mm} \\
\mathrm{f}_{\mathrm{i}, \mathrm{Q}, \lim }: 10.43 \mathrm{~mm}\end{array}$ & $\begin{array}{l}\mathrm{f}_{\mathrm{T}, \max }: 0.24 \mathrm{~mm} \\
\mathrm{f}_{\mathrm{T}, \mathrm{lim}}: 14.60 \mathrm{~mm}\end{array}$ & $\begin{array}{l}\mathrm{f}_{\mathrm{A}, \max }: 0.20 \mathrm{~mm} \\
\mathrm{f}_{\mathrm{A}, \mathrm{lim}}: 7.30 \mathrm{~mm}\end{array}$ & PASSA \\
\hline c: P6 - P3 & $\begin{array}{l}f_{\mathrm{i}, \mathrm{Q}}: 0.00 \mathrm{~mm} \\
\mathrm{f}_{\mathrm{i}, \mathrm{Q}, \lim }: 8.00 \mathrm{~mm}\end{array}$ & $\begin{array}{l}\mathrm{f}_{\mathrm{T}, \max }: 0.01 \mathrm{~mm} \\
\mathrm{f}_{\mathrm{T}, \lim }: 3.41 \mathrm{~mm}\end{array}$ & $\begin{array}{l}f_{A, \max }: 0.02 \mathrm{~mm} \\
f_{A, l i m}: 2.10 \mathrm{~mm}\end{array}$ & PASSA \\
\hline
\end{tabular}

\section{Cobertura}

\begin{tabular}{|c|c|c|c|c|c|c|c|c|c|c|c|c|c|c|c|c|}
\hline \multirow{2}{*}{ Vigas } & \multicolumn{15}{|c|}{ VERIFICAÇÕES DE RESISTÊNCIA (ABNT NBR 6118:2014) } & \multirow{2}{*}{ Estado } \\
\hline & Disp. & Arm. & $\mathrm{Q}$ & $N, M$ & \begin{tabular}{|l|}
$T_{c}$ \\
\end{tabular} & $\mathrm{~T}_{\text {st }}$ & $\mathrm{T}_{\mathrm{sl}}$ & TNM $_{\mathrm{x}}$ & $\mathrm{TV}_{\mathrm{x}}$ & $\mathrm{TV}_{\mathrm{y}}$ & TV $\mathrm{XS}_{\mathrm{t}}$ & TV $\mathrm{YS}_{\mathrm{t}}$ & T,Disp.sl & T, Geom.st & $\mathrm{T}, \mathrm{Arm}$.st & \\
\hline a: P1 - P2 & Passa & Passa & $\begin{array}{l}' 4.394 \mathrm{~m} ' \\
\eta=38.9\end{array}$ & $\begin{array}{l}14.270 \mathrm{~m}^{\prime} \\
\eta=82.7\end{array}$ & N.P. ${ }^{(1)}$ & N.P. ${ }^{(1)}$ & N.P. ${ }^{(1)}$ & N.P. ${ }^{(2)}$ & N.P. ${ }^{(1)}$ & N.P. ${ }^{(1)}$ & N.P. ${ }^{(1)}$ & N.P. ${ }^{(1)}$ & N.P. ${ }^{(1)}$ & N.P. ${ }^{(1)}$ & N.P. ${ }^{(1)}$ & $\begin{array}{c}\text { PASSA } \\
\eta=82.7\end{array}$ \\
\hline b: P2 - P3 & Passa & Passa & $\begin{array}{l}10.257 \mathrm{~m} \\
\eta=36.5\end{array}$ & $\begin{array}{c}\text { 'P2' } \\
\eta=82.0\end{array}$ & N.P. (1) & N.P. ${ }^{(1)}$ & N.P. ${ }^{(1)}$ & N.P. ${ }^{(2)}$ & N.P. ${ }^{(1)}$ & N.P. ${ }^{(1)}$ & N.P. ${ }^{(1)}$ & N.P. ${ }^{(1)}$ & N.P. ${ }^{(1)}$ & N.P. ${ }^{(1)}$ & N.P. (1) & $\begin{array}{c}\text { PASSA } \\
\eta=82.0\end{array}$ \\
\hline
\end{tabular}




\begin{tabular}{|c|c|c|c|c|c|c|c|c|c|c|c|c|c|c|c|c|}
\hline \multirow{2}{*}{ Vigas } & \multicolumn{15}{|c|}{ VERIFICAÇÕES DE RESISTÊNCIA (ABNT NBR 6118:2014) } & \multirow{2}{*}{ Estado } \\
\hline & Disp. & Arm. & $\mathrm{Q}$ & $\mathrm{N}, \mathrm{M}$ & \begin{tabular}{|l|}
$T_{c}$ \\
\end{tabular} & \begin{tabular}{|l|} 
st \\
\end{tabular} & $\mathrm{T}_{\mathrm{sl}}$ & TNM $_{x}$ & $T V_{x}$ & $\mathrm{TV}_{\mathrm{y}}$ & TVXSt & TVYSt & T,Disp.si & T,Geom.st & T,Arm.st & \\
\hline a: P5 - P6 & Passa & Passa & $\begin{array}{l}0.207 \mathrm{~m}^{\prime} \\
\eta=74.0 \\
\end{array}$ & $\begin{array}{l}\prime 1.915 \mathrm{~m}^{\prime} \\
\eta=89.6\end{array}$ & N.P. ${ }^{(1)}$ & N.P. ${ }^{(1)}$ & N.P. ${ }^{(1)}$ & N.P. ${ }^{(2)}$ & N.P. ${ }^{(1)}$ & N.P. ${ }^{(1)}$ & N.P. ${ }^{(1)}$ & N.P. ${ }^{(1)}$ & N.P. ${ }^{(1)}$ & N.P. ${ }^{(1)}$ & N.P. ${ }^{(1)}$ & $\begin{array}{c}\text { PASSA } \\
\eta=89.6\end{array}$ \\
\hline a: P7 - P8 & Passa & Passa & $\begin{array}{l}0.207 \mathrm{~m}^{\prime} \\
\eta=48.5 \\
\end{array}$ & $\begin{array}{l}\prime 1.800 \mathrm{~m} \\
\eta=88.0\end{array}$ & N.P. (1) & N.P. ${ }^{(1)}$ & N.P. ${ }^{(1)}$ & N.P. ${ }^{(2)}$ & N.P. ${ }^{(1)}$ & N.P. ${ }^{(1)}$ & N.P. ${ }^{(1)}$ & N.P. ${ }^{(1)}$ & N.P. ${ }^{(1)}$ & N.P. ${ }^{(1)}$ & N.P. ${ }^{(1)}$ & $\begin{array}{c}\text { PASSA } \\
\eta=88.0\end{array}$ \\
\hline a: P10 - P11 & Passa & Passa & $\begin{array}{l}4.044 \mathrm{~m}^{\prime} \\
\eta=56.0\end{array}$ & $\begin{array}{l}44.044 \mathrm{~m}^{\prime} \\
\eta=88.1\end{array}$ & N.P. (1) & N.P. ${ }^{(1)}$ & N.P. ${ }^{(1)}$ & N.P. ${ }^{(2)}$ & N.P. ${ }^{(1)}$ & N.P. ${ }^{(1)}$ & N.P. ${ }^{(1)}$ & N.P. ${ }^{(1)}$ & N.P. ${ }^{(1)}$ & N.P. ${ }^{(1)}$ & N.P. ${ }^{(1)}$ & $\begin{array}{c}\text { PASSA } \\
\eta=88.1\end{array}$ \\
\hline b: $\mathrm{P} 11-\mathrm{P} 12$ & Passa & Passa & $\begin{array}{l}0.207 \mathrm{~m} \\
\eta=68.9\end{array}$ & $\begin{array}{l}2 \\
2.290 \mathrm{~m} \\
\eta=88.8\end{array}$ & N.P. ${ }^{(1)}$ & N.P. ${ }^{(1)}$ & N.P. ${ }^{(1)}$ & N.P. ${ }^{(2)}$ & N.P. ${ }^{(1)}$ & N.P. ${ }^{(1)}$ & N.P. ${ }^{(1)}$ & N.P. ${ }^{(1)}$ & N.P. ${ }^{(1)}$ & N.P. ${ }^{(1)}$ & N.P. ${ }^{(1)}$ & $\begin{array}{c}\text { PASSA } \\
\eta=88.8 \\
\end{array}$ \\
\hline a: P14 - P15 & Passa & Passa & $\begin{array}{l}4.019 \mathrm{~m}^{\prime} \\
\eta=42.4\end{array}$ & $\begin{array}{l}4.019 \mathrm{~m} \\
\eta=90.7\end{array}$ & N.P. (1) & N.P. ${ }^{(1)}$ & N.P. ${ }^{(1)}$ & N.P. ${ }^{(2)}$ & N.P. ${ }^{(1)}$ & N.P. ${ }^{(1)}$ & N.P. ${ }^{(1)}$ & N.P. ${ }^{(1)}$ & N.P. ${ }^{(1)}$ & N.P. ${ }^{(1)}$ & N.P. ${ }^{(1)}$ & $\begin{array}{c}\text { PASSA } \\
\eta=90.7\end{array}$ \\
\hline b: P15 - P16 & Passa & Passa & $\begin{array}{l}0.207 \mathrm{~m}^{\prime} \\
\eta=46.9 \\
\end{array}$ & $\begin{array}{l}\prime 2.265 \mathrm{~m}^{\prime} \\
\eta=93.3\end{array}$ & N.P. (1) & N.P. (1) & N.P. ${ }^{(1)}$ & N.P. (2) & N.P. (1) & N.P. (1) & N.P. ${ }^{(1)}$ & N.P. ${ }^{(1)}$ & N.P. $(1)$ & N.P. ${ }^{(1)}$ & N.P. ${ }^{(1)}$ & $\begin{array}{c}\text { PASSA } \\
\eta=93.3\end{array}$ \\
\hline a: P14 - P10 & Passa & Passa & $\begin{array}{c}4.044 \mathrm{~m}^{\prime} \\
\eta=8.4 \\
\end{array}$ & $\begin{array}{l}\prime 1.518 \mathrm{~m} \\
\eta=19.2\end{array}$ & N.P. (1) & N.P. (1) & N.P. ${ }^{(1)}$ & N.P. (2) & N.P. (1) & N.P. ${ }^{(1)}$ & N.P. ${ }^{(1)}$ & N.P. ${ }^{(1)}$ & N.P. ${ }^{(1)}$ & N.P. ${ }^{(1)}$ & N.P. ${ }^{(1)}$ & $\begin{array}{c}\text { PASSA } \\
\eta=19.2\end{array}$ \\
\hline b: P10 - P7 & Passa & Passa & $\begin{array}{c}0.207 \mathrm{~m}^{\prime} \\
\eta=4.3 \\
\end{array}$ & $\begin{array}{c}P 10 ' \\
\eta=15.9\end{array}$ & N.P. ${ }^{(1)}$ & N.P. (1) & N.P. ${ }^{(1)}$ & N.P. (2) & N.P. (1) & N.P. (1) & N.P. (1) & N.P. ${ }^{(1)}$ & N.P. ${ }^{(1)}$ & N.P. ${ }^{(1)}$ & N.P. ${ }^{(1)}$ & $\begin{array}{c}\text { PASSA } \\
\eta=15.9\end{array}$ \\
\hline b: P11 - P8 & Passa & Passa & $\begin{array}{c}0.207 \mathrm{~m}^{\prime} \\
\eta=9.6\end{array}$ & $\begin{array}{l}\prime 2.166 \mathrm{~m}^{\prime} \\
\eta=24.2\end{array}$ & N.P. (1) & N.P. ${ }^{(1)}$ & N.P. ${ }^{(1)}$ & N.P. (2) & N.P. ${ }^{(1)}$ & N.P. ${ }^{(1)}$ & N.P. ${ }^{(1)}$ & N.P. ${ }^{(1)}$ & N.P. ${ }^{(1)}$ & N.P. ${ }^{(1)}$ & N.P. ${ }^{(1)}$ & $\begin{array}{c}\text { PASSA } \\
\eta=24.2\end{array}$ \\
\hline c: P8 - P5 & Passa & Passa & $\begin{array}{l}0.819 \mathrm{~m}^{\prime} \\
\eta=17.2\end{array}$ & $\begin{array}{l}10.769 \mathrm{~m} \\
\eta=29.7\end{array}$ & N.P. (1) & N.P. ${ }^{(1)}$ & N.P. ${ }^{(1)}$ & N.P. (2) & N.P. (1) & N.P. ${ }^{(1)}$ & N.P. ${ }^{(1)}$ & N.P. ${ }^{(1)}$ & N.P. ${ }^{(1)}$ & N.P. ${ }^{(1)}$ & N.P. ${ }^{(1)}$ & $\begin{array}{c}\text { PASSA } \\
\eta=29.7\end{array}$ \\
\hline
\end{tabular}

\begin{tabular}{|c|c|c|c|c|c|c|c|c|c|c|c|c|c|c|c|c|c|}
\hline \multirow{2}{*}{ Vigas } & \multicolumn{16}{|c|}{ VERIFICAÇÕES DE RESISTÊNCIA (ABNT NBR 6118:2014) } & \multirow{2}{*}{ Estado } \\
\hline & Disp. & Arm. & Q & $\mathrm{N}, \mathrm{M}$ & $\mathrm{T}_{\mathrm{c}}$ & $\mathrm{T}_{\text {st }}$ & $\mathrm{T}_{\mathrm{sl}}$ & TNM $_{x}$ & $\mathrm{TV}_{\mathrm{x}}$ & $\mathrm{TV}_{\mathrm{y}}$ & TVxSt & TVYSt & T,Disp.s! & T,Geom.st & T,Arm.st & t- & \\
\hline C: P7 - P1 & Passa & Passa & $\begin{array}{c}' 0.207 \mathrm{~m}^{\prime} \\
\eta=7.3\end{array}$ & $\begin{array}{l}\text { ' } 1.656 \mathrm{~m} ' \\
\eta=16.2\end{array}$ & N.P. ${ }^{(1)}$ & N.P. ${ }^{(1)}$ & N.P. (1) & N.P. (2) & N.P. ${ }^{(1)}$ & N.P. ${ }^{(1)}$ & N.P. (1) & N.P. ${ }^{(1)}$ & N.P. ${ }^{(1)}$ & N.P. ${ }^{(1)}$ & N.P. ${ }^{(1)}$ & N.P. ${ }^{(3)}$ & $\begin{array}{c}\text { PASSA } \\
\eta=16.2\end{array}$ \\
\hline a: P15 - P11 & Passa & Passa & & & N.P. ${ }^{(1)}$ & N.P. ${ }^{(1)}$ & N.P. ${ }^{(1)}$ & N.P. (2) & N.P. ${ }^{(1)}$ & N.P. ${ }^{(1)}$ & N.P. ${ }^{(1)}$ & N.P. ${ }^{(1)}$ & N.P. ${ }^{(1)}$ & & N.P. ${ }^{(1)}$ & N.P. ${ }^{(3)}$ & \\
\hline a: P5 - P2 & Passa & Passa & $\begin{array}{c}' 0.207 \mathrm{~m}^{\prime} \\
\eta=8.2\end{array}$ & $\begin{array}{c}\text { 'P5' } \\
\eta=21.7\end{array}$ & N.P. ${ }^{(1)}$ & N.P. ${ }^{(1)}$ & N.P. (1) & N.P. (2) & N.P. ${ }^{(1)}$ & N.P. ${ }^{(1)}$ & N.P. ${ }^{(1)}$ & N.P. ${ }^{(1)}$ & N.P. ${ }^{(1)}$ & & N.P. ${ }^{(1)}$ & N.P. ${ }^{(3)}$ & \\
\hline a: P16 - P12 & Passa & Passa & $\begin{array}{c}' 4.044 \mathrm{~m}^{\prime} \\
\eta=7.8\end{array}$ & $\begin{array}{l}\text { '3.946 m' } \\
\eta=19.6\end{array}$ & N.P. ${ }^{(1)}$ & N.P. ${ }^{(1)}$ & N.P. ${ }^{(1)}$ & N.P. (2) & N.P. ${ }^{(1)}$ & N.P. ${ }^{(1)}$ & N.P. ${ }^{(1)}$ & N.P. ${ }^{(1)}$ & N.P. ${ }^{(1)}$ & & N.P. ${ }^{(1)}$ & N.P. (3) & \\
\hline b: P12 - P6 & Passa & Passa & $\begin{array}{c}' 0.207 \mathrm{~m}^{\prime} \\
\eta=6.5\end{array}$ & $\begin{array}{c}\text { 'P12' } \\
\eta=20.5\end{array}$ & N.P. ${ }^{(1)}$ & N.P. ${ }^{(1)}$ & N.P. ${ }^{(1)}$ & N.P. (2) & N.P. ${ }^{(1)}$ & N.P. ${ }^{(1)}$ & N.P. ${ }^{(1)}$ & N.P. ${ }^{(1)}$ & N.P. ${ }^{(1)}$ & N.P..$^{(1)}$ & N.P. ${ }^{(1)}$ & N.P. ${ }^{(3)}$ & $\begin{array}{c}\text { PASSA } \\
\eta=20.5\end{array}$ \\
\hline c: P6 - P3 & Passa & Passa & $\begin{array}{c}' 0.207 \mathrm{~m}^{\prime} \\
\eta=3.2\end{array}$ & $\begin{array}{c}\text { 'P6' } \\
\eta=9.9\end{array}$ & N.P. ${ }^{(1)}$ & N.P. ${ }^{(1)}$ & N.P. ${ }^{(1)}$ & N.P. (2) & N.P. ${ }^{(1)}$ & N.P. ${ }^{(1)}$ & N.P. ${ }^{(1)}$ & N.P. ${ }^{(1)}$ & N.P. ${ }^{(1)}$ & N.P..$^{(1)}$ & N.P..$^{(1)}$ & N.P. ${ }^{(3)}$ & $\begin{array}{c}\text { PASSA } \\
\eta=9.9\end{array}$ \\
\hline
\end{tabular}

\footnotetext{
Notação:

Disp.: Disposições relativas às armaduras

Arm.: Armadura mínima e máxima

Q: Estado limite de ruptura relativo ao esforço cortante (combinações não sísmicas)

N,M: Estado limite de ruptura frente a solicitações normais (combinações não sísmicas)

$T_{c}$ : Estado limite de ruptura por torção. Compressão oblíqua.

$T_{\text {st: }}$ Estado limite de ruptura por torção. Tração na alma.

$T_{s l}$ : Estado limite de ruptura por torção. Tração nas armaduras longitudinais.

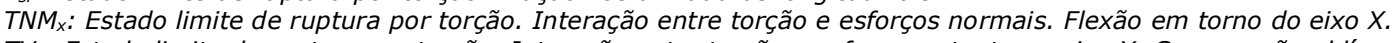
$T V_{x}$ : Estado limite de ruptura por torção. Interação entre torção e esforço cortante no eixo $X$. Compressão oblíqua $T V_{y}$ : Estado limite de ruptura por torção. Interação entre torção e esforço cortante no eixo Y. Compressão oblíqua $T V_{x} S_{t}:$ Estado limite de ruptura por torção. Interação entre torção e esforço cortante no eixo X. Tração na alma. TVYSt: Estado limite de ruptura por torção. Interação entre torção e esforço cortante no eixo Y. Tração na alma. T,Disp.sl: Estado limite de ruptura por torção. Espaçamento entre as barras da armadura longitudinal.

T,Geom.st: Estado limite de ruptura por torção. Diâmetro mínimo da armadura transversal.

T,Arm.st: Estado limite de ruptura por torção. Quantidade mínima de estribos fechados.

x: Distância à origem da barra

$\eta$ : Coeficiente de aproveitamento (\%)

N.P.: Não procede

$-:-$

Verificações desnecessárias para o tipo de perfil (N.P.):

(1) A verificação do estado limite de ruptura por torção não é necessária, já que não há momento de torção.

(2) A verificação não é necessária, já que não há interação entre torção e esforços normais.

(3) Não há esforços que produzam tensões normais para nenhuma combinação. Portanto, a verificação não é necessária.
} 


\section{Pontifícia Universidade Cháólica} DO RIO DE JANEIRO

\begin{tabular}{|c|c|c|c|c|c|c|}
\hline \multirow{2}{*}{ Vigas } & \multicolumn{5}{|c|}{ VERIFICAÇÕES DE FISSURAÇÃO (ABNT NBR 6118:2014) } & \multirow{2}{*}{ Estado } \\
\hline & $W_{k, F, \text { sup. }}$ & $\mathrm{W}_{\mathrm{k}, \mathrm{F}, \text { Lat.Dir. }}$ & $W_{k, F, \text { inf. }}$ & $\mathrm{W}_{\mathrm{k}, \mathrm{F} \text {, Lat.Esq. }}$ & $\sigma_{\mathrm{s}}$ & \\
\hline a: $\mathrm{P} 1-\mathrm{P} 2$ & $\begin{array}{c}\mathrm{x}: 4.65 \mathrm{~m} \\
\text { Passa }\end{array}$ & N.P. ${ }^{(1)}$ & $\begin{array}{c}\mathrm{x}: 1.8 \mathrm{~m} \\
\text { Passa }\end{array}$ & N.P. ${ }^{(1)}$ & N.P. $(2)$ & PASSA \\
\hline b: $\mathrm{P} 2-\mathrm{P} 3$ & $\begin{array}{l}\text { x: } 0 \text { m } \\
\text { Passa }\end{array}$ & N.P. $(1)$ & $\begin{array}{c}\mathrm{x}: 2.65 \mathrm{~m} \\
\text { Passa }\end{array}$ & N.P. ${ }^{(1)}$ & N.P. $(2)$ & PASSA \\
\hline a: P5 - P6 & $\begin{array}{l}\mathrm{x}: 0 \mathrm{~m} \\
\text { Passa }\end{array}$ & N.P.(1) & 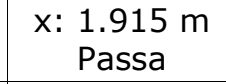 & N.P. ${ }^{(1)}$ & $\begin{array}{c}\mathrm{x}: 0.965 \mathrm{~m} \\
\text { Passa }\end{array}$ & PASSA \\
\hline a: P7 - P8 & $\begin{array}{c}\mathrm{x}: 4.25 \mathrm{~m} \\
\text { Passa }\end{array}$ & N.P. ${ }^{(3)}$ & $\begin{array}{c}\mathrm{x}: 2.18 \mathrm{~m} \\
\text { Passa }\end{array}$ & N.P. ${ }^{(3)}$ & $\begin{array}{c}\mathrm{x}: 1.04 \mathrm{~m} \\
\text { Passa }\end{array}$ & PASSA \\
\hline a: P10 - P11 & $\begin{array}{c}\mathrm{x}: 4.25 \mathrm{~m} \\
\text { Passa }\end{array}$ & N.P. $(1)$ & $\begin{array}{c}\mathrm{x}: 1.42 \mathrm{~m} \\
\text { Passa }\end{array}$ & N.P. ${ }^{(1)}$ & $\begin{array}{c}\mathrm{x}: 4.044 \mathrm{~m} \\
\text { Passa }\end{array}$ & PASSA \\
\hline b: P11 - P12 & $\begin{array}{l}\mathrm{x}: 0 \mathrm{~m} \\
\text { Passa }\end{array}$ & N.P. ${ }^{(1)}$ & $\begin{array}{c}x: 2.67 \mathrm{~m} \\
\text { Passa }\end{array}$ & N.P. ${ }^{(1)}$ & $\begin{array}{c}\mathrm{x}: 2.1 \mathrm{~m} \\
\text { Passa }\end{array}$ & PASSA \\
\hline a: P14 - P15 & $\begin{array}{c}\mathrm{x}: 4.225 \mathrm{~m} \\
\text { Passa }\end{array}$ & N.P. ${ }^{(1)}$ & $\begin{array}{c}\mathrm{x}: 1.61 \mathrm{~m} \\
\text { Passa }\end{array}$ & N.P. ${ }^{(1)}$ & $\begin{array}{c}\mathrm{x}: 4.153 \mathrm{~m} \\
\text { Passa }\end{array}$ & PASSA \\
\hline b: P15 - P16 & $\begin{array}{c}\text { x: } 0 \mathrm{~m} \\
\text { Passa }\end{array}$ & N.P. ${ }^{(1)}$ & $\begin{array}{c}\mathrm{x}: 2.645 \mathrm{~m} \\
\text { Passa }\end{array}$ & N.P. ${ }^{(1)}$ & $\begin{array}{c}\text { x: } 0 \mathrm{~m} \\
\text { Passa }\end{array}$ & PASSA \\
\hline a: P14 - P10 & $\begin{array}{c}\mathrm{x}: 4.25 \mathrm{~m} \\
\text { Passa }\end{array}$ & N.P. ${ }^{(3)}$ & $\begin{array}{l}\mathrm{x}: 1.821 \mathrm{~m} \\
\text { Passa }\end{array}$ & N.P. ${ }^{(3)}$ & N.P. (3) & PASSA \\
\hline b: P10 - P7 & $\begin{array}{l}\text { x: } 0 \text { m } \\
\text { Passa }\end{array}$ & N.P. ${ }^{(1)}$ & N.P. ${ }^{(1)}$ & N.P. ${ }^{(1)}$ & N.P. ${ }^{(2)}$ & PASSA \\
\hline b: $P 11-P 8$ & $\begin{array}{l}\text { x: } 0 \mathrm{~m} \\
\text { Passa }\end{array}$ & N.P. ${ }^{(1)}$ & 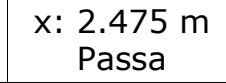 & N.P. ${ }^{(1)}$ & N.P. ${ }^{(2)}$ & PASSA \\
\hline c: P8 - P5 & $\begin{array}{c}\mathrm{x}: 1.025 \mathrm{~m} \\
\text { Passa }\end{array}$ & N.P. ${ }^{(1)}$ & $\begin{array}{c}\mathrm{x}: 0 \mathrm{~m} \\
\text { Passa }\end{array}$ & N.P. ${ }^{(1)}$ & N.P. ${ }^{(2)}$ & PASSA \\
\hline c: P6 - P3 & $\begin{array}{l}\text { x: } 0 \mathrm{~m} \\
\text { Passa }\end{array}$ & N.P. ${ }^{(1)}$ & $\begin{array}{c}\mathrm{x}: 1.75 \mathrm{~m} \\
\text { Passa }\end{array}$ & N.P. ${ }^{(1)}$ & N.P. ${ }^{(2)}$ & PASSA \\
\hline
\end{tabular}

\begin{tabular}{|c|c|c|c|c|c|c|c|}
\hline \multirow{2}{*}{ Vigas } & \multicolumn{6}{|c|}{ VERIFICAÇÕES DE FISSURAÇÃO (ABNT NBR 6118:2014) } & \multirow{2}{*}{ Estado } \\
\hline & $W_{k, F, \text { sup }}$ & $W_{\mathrm{k}, \mathrm{F} \text {, Lat.Dir. }}$ & $W_{k, F, \text { inf. }}$ & $\mathrm{W}_{\mathrm{k}, \mathrm{F}, \text { Lat.Esq. }}$ & $\sigma_{\mathrm{s}}$ & - & \\
\hline C: P7 - P1 & $\begin{array}{l}\text { x: } 0 \mathrm{~m} \\
\text { Passa }\end{array}$ & N.P. ${ }^{(1)}$ & $\begin{array}{c}\mathrm{x}: 1.988 \mathrm{~m} \\
\text { Passa }\end{array}$ & N.P. ${ }^{(1)}$ & N.P. ${ }^{(2)}$ & N.P. ${ }^{(3)}$ & PASSA \\
\hline a: P15 - P11 & $\begin{array}{c}\mathrm{x}: 4.25 \mathrm{~m} \\
\text { Passa }\end{array}$ & N.P. ${ }^{(1)}$ & $\begin{array}{c}\mathrm{x}: 1.821 \mathrm{~m} \\
\text { Passa }\end{array}$ & N.P. ${ }^{(1)}$ & N.P. (2) & N.P. (3) & PASSA \\
\hline $\mathrm{a}: \mathrm{P5}-\mathrm{P2}$ & $\begin{array}{l}\text { x: } 0 \mathrm{~m} \\
\text { Passa }\end{array}$ & N.P. ${ }^{(1)}$ & $\begin{array}{c}\mathrm{x}: 1.05 \mathrm{~m} \\
\text { Passa }\end{array}$ & N.P. ${ }^{(1)}$ & N.P. ${ }^{(2)}$ & N.P. ${ }^{(3)}$ & PASSA \\
\hline a: P16 - P12 & $\begin{array}{c}\mathrm{x}: 4.25 \mathrm{~m} \\
\text { Passa }\end{array}$ & N.P. ${ }^{(1)}$ & $\mathrm{x}: \begin{array}{l}1.821 \mathrm{~m} \\
\text { Passa }\end{array}$ & N.P. ${ }^{(1)}$ & N.P. ${ }^{(2)}$ & N.P. ${ }^{(3)}$ & PASSA \\
\hline b: P12 - P6 & $\begin{array}{l}\text { x: } 0 \mathrm{~m} \\
\text { Passa }\end{array}$ & N.P. ${ }^{(1)}$ & $\begin{array}{c}\mathrm{x}: 2.129 \mathrm{~m} \\
\text { Passa }\end{array}$ & N.P. ${ }^{(1)}$ & N.P. ${ }^{(2)}$ & N.P. ${ }^{(3)}$ & PASSA \\
\hline
\end{tabular}

$W_{k, F, \text { sup. }}$ Controle da fissuração através da limitação da abertura estimada das fissuras: Face superior $W_{k, F, \text { Lat.Dir. }}$ Controle da fissuração através da limitação da abertura estimada das fissuras: Face lateral direita $W_{k, F, \text { inf. }}$ Controle da fissuração através da limitação da abertura estimada das fissuras: Face inferior

$W_{k, F, \text { Lat.Esq. }}$ Controle da fissuração através da limitação da abertura estimada das fissuras: Face lateral esquerda $\sigma_{s}$ : Armaduras longitudinais mínimas

x: Distância à origem da barra

$\eta$ : Coeficiente de aproveitamento (\%)

N.P.: Não procede

$-:-$
} 
Verificações desnecessárias para o tipo de perfil (N.P.):

(1) A verificação não é necessária, já que não há nenhuma armadura tracionada.

(2) A verificação não é necessária, já que a tensão de tração máxima no concreto não supera a resistência à tração do mesmo.

(3) Não há esforços que produzam tensões normais para nenhuma combinação. Portanto, a verificação não é necessária.

\begin{tabular}{|c|c|c|c|c|}
\hline \multicolumn{5}{|c|}{ Verificações de flecha } \\
\hline Vigas & $\begin{array}{c}\text { Sobrecarga } \\
\text { (Característica) } \\
\mathrm{f}_{\mathrm{i}, \mathrm{Q}} \leq \mathrm{f}_{\mathrm{i}, \mathrm{Q}, \lim } \\
\mathrm{f}_{\mathrm{i}, \mathrm{Q}, \lim }=\mathrm{L} / 350\end{array}$ & $\begin{array}{c}\text { No tempo infinito } \\
\text { (Quase permanente) } \\
\mathrm{f}_{\mathrm{T}, \max } \leq \mathrm{f}_{\mathrm{T}, \lim } \\
\mathrm{f}_{\mathrm{T}, \lim }=\mathrm{L} / 250\end{array}$ & $\begin{array}{c}\text { Ativa } \\
\text { (Característica) } \\
\mathrm{f}_{\mathrm{A}, \max } \leq \mathrm{f}_{\mathrm{A}, \lim } \\
\mathrm{f}_{\mathrm{A}, \lim }=\text { Mín. }(10.00, \mathrm{~L} / 500)\end{array}$ & Estado \\
\hline a: P1 - P2 & $\begin{array}{l}f_{i, Q}: 0.16 \mathrm{~mm} \\
f_{i, Q}, \lim : 13.29 \mathrm{~mm}\end{array}$ & $\begin{array}{l}\mathrm{f}_{\mathrm{T}, \max }: 0.94 \mathrm{~mm} \\
\mathrm{f}_{\mathrm{T}, \mathrm{lim}}: 18.60 \mathrm{~mm}\end{array}$ & $\begin{array}{l}\mathrm{f}_{\mathrm{A}, \max }: 0.66 \mathrm{~mm} \\
\mathrm{f}_{\mathrm{A}, \mathrm{lim}}: 9.30 \mathrm{~mm}\end{array}$ & PASSA \\
\hline b: P2 - P3 & $\begin{array}{l}f_{i, Q}: 0.03 \mathrm{~mm} \\
f_{i, Q}, \lim : 9.38 \mathrm{~mm}\end{array}$ & $\begin{array}{l}\mathrm{f}_{\mathrm{T}, \max }: 0.43 \mathrm{~mm} \\
\mathrm{f}_{\mathrm{T}, \lim }: 16.34 \mathrm{~mm}\end{array}$ & $\begin{array}{l}\mathrm{f}_{\mathrm{A}, \max }: 0.32 \mathrm{~mm} \\
\mathrm{f}_{\mathrm{A}, \lim }: 8.23 \mathrm{~mm}\end{array}$ & PASSA \\
\hline a: P5 - P6 & $\begin{array}{l}f_{i, Q}: 2.10 \mathrm{~mm} \\
f_{i, Q}, \lim : 12.36 \mathrm{~mm}\end{array}$ & $\begin{array}{l}\mathrm{f}_{\mathrm{T}, \max }: 5.52 \mathrm{~mm} \\
\mathrm{f}_{\mathrm{T}, \lim }: 17.30 \mathrm{~mm}\end{array}$ & $\begin{array}{l}\mathrm{f}_{\mathrm{A}, \max }: 5.57 \mathrm{~mm} \\
\mathrm{f}_{\mathrm{A}, \lim }: 8.65 \mathrm{~mm}\end{array}$ & PASSA \\
\hline a: P7 - P8 & $\begin{array}{l}f_{i, Q}: 3.37 \mathrm{~mm} \\
f_{i, Q}, \lim : 12.14 \mathrm{~mm}\end{array}$ & $\begin{array}{l}\mathrm{f}_{\mathrm{T}, \max }: 6.50 \mathrm{~mm} \\
\mathrm{f}_{\mathrm{T}, \lim }: 17.00 \mathrm{~mm}\end{array}$ & $\begin{array}{l}\mathrm{f}_{\mathrm{A}, \max }: 6.53 \mathrm{~mm} \\
\mathrm{f}_{\mathrm{A}, \lim }: 8.50 \mathrm{~mm}\end{array}$ & PASSA \\
\hline a: P10 - P11 & $\begin{array}{l}f_{i, Q}: 0.22 \mathrm{~mm} \\
f_{i, Q}, \lim : 10.87 \mathrm{~mm}\end{array}$ & $\begin{array}{l}\mathrm{f}_{\mathrm{T}, \max }: 0.99 \mathrm{~mm} \\
\mathrm{f}_{\mathrm{T}, \lim }: 15.49 \mathrm{~mm}\end{array}$ & $\begin{array}{l}\mathrm{f}_{\mathrm{A}, \max }: 0.68 \mathrm{~mm} \\
\mathrm{f}_{\mathrm{A}, \lim }: 7.74 \mathrm{~mm}\end{array}$ & PASSA \\
\hline b: P11 - P12 & $\begin{array}{l}\mathrm{f}_{\mathrm{i}, \mathrm{Q}}: 1.85 \mathrm{~mm} \\
\mathrm{f}_{\mathrm{i}, \mathrm{Q}, \lim : 13.43 \mathrm{~mm}}\end{array}$ & $\begin{array}{l}\mathrm{f}_{\mathrm{T}, \max }: 3.82 \mathrm{~mm} \\
\mathrm{f}_{\mathrm{T}, \lim }: 18.80 \mathrm{~mm}\end{array}$ & $\begin{array}{l}\mathrm{f}_{\mathrm{A}, \max }: 3.68 \mathrm{~mm} \\
\mathrm{f}_{\mathrm{A}, \lim }: 9.40 \mathrm{~mm}\end{array}$ & PASSA \\
\hline a: P14 - P15 & $\begin{array}{l}f_{i, Q}: 0.15 \mathrm{~mm} \\
f_{i, Q}, \lim : 11.65 \mathrm{~mm}\end{array}$ & $\begin{array}{l}\mathrm{f}_{\mathrm{T}, \max }: 0.89 \mathrm{~mm} \\
\mathrm{f}_{\mathrm{T}, \lim }: 16.27 \mathrm{~mm}\end{array}$ & $\begin{array}{l}\mathrm{f}_{\mathrm{A}, \max }: 0.67 \mathrm{~mm} \\
\mathrm{f}_{\mathrm{A}, \lim }: 8.14 \mathrm{~mm}\end{array}$ & PASSA \\
\hline b: P15 - P16 & $\begin{array}{l}f_{i, Q}: 0.27 \mathrm{~mm} \\
f_{i, Q}, \lim : 13.36 \mathrm{~mm}\end{array}$ & $\begin{array}{l}\mathrm{f}_{\mathrm{T}, \max }: 1.58 \mathrm{~mm} \\
\mathrm{f}_{\mathrm{T}, \lim }: 18.70 \mathrm{~mm}\end{array}$ & $\begin{array}{l}\mathrm{f}_{\mathrm{A}, \max }: 1.20 \mathrm{~mm} \\
\mathrm{f}_{\mathrm{A}, \lim }: 9.35 \mathrm{~mm}\end{array}$ & PASSA \\
\hline a: P14 - P10 & $\begin{array}{l}\mathrm{f}_{\mathrm{i}, \mathrm{Q}}: 0.01 \mathrm{~mm} \\
\mathrm{f}_{\mathrm{i}, \mathrm{Q}, \lim : 12.14 \mathrm{~mm}}\end{array}$ & $\begin{array}{l}\mathrm{f}_{\mathrm{T}, \max }: 0.35 \mathrm{~mm} \\
\mathrm{f}_{\mathrm{T}, \lim }: 17.00 \mathrm{~mm}\end{array}$ & $\begin{array}{l}\mathrm{f}_{\mathrm{A}, \max }: 0.15 \mathrm{~mm} \\
\mathrm{f}_{\mathrm{A}, \lim }: 8.50 \mathrm{~mm}\end{array}$ & PASSA \\
\hline $\mathrm{b}: \mathrm{P} 10-\mathrm{P} 7$ & $\begin{array}{l}f_{i, Q}: 0.00 \mathrm{~mm} \\
f_{i, Q}, \lim : 7.07 \mathrm{~mm}\end{array}$ & $\begin{array}{l}\mathrm{f}_{\mathrm{T}, \max }: 0.07 \mathrm{~mm} \\
\mathrm{f}_{\mathrm{T}, \lim }: 9.90 \mathrm{~mm}\end{array}$ & $\begin{array}{l}\mathrm{f}_{\mathrm{A}, \max }: 0.03 \mathrm{~mm} \\
\mathrm{f}_{\mathrm{A}, \lim }: 4.95 \mathrm{~mm}\end{array}$ & PASSA \\
\hline c: P7 - P1 & $\begin{array}{l}f_{i, Q}: 0.01 \mathrm{~mm} \\
f_{i, Q}, \lim : 11.36 \mathrm{~mm}\end{array}$ & $\begin{array}{l}\mathrm{f}_{\mathrm{T}, \max }: 0.27 \mathrm{~mm} \\
\mathrm{f}_{\mathrm{T}, \lim }: 15.90 \mathrm{~mm}\end{array}$ & $\begin{array}{l}\mathrm{f}_{\mathrm{A}, \max }: 0.11 \mathrm{~mm} \\
\mathrm{f}_{\mathrm{A}, \lim }: 7.95 \mathrm{~mm}\end{array}$ & PASSA \\
\hline a: P15 - P11 & $\begin{array}{l}f_{i, Q}: 0.02 \mathrm{~mm} \\
f_{i, Q, \lim }: 12.14 \mathrm{~mm}\end{array}$ & $\begin{array}{l}\mathrm{f}_{\mathrm{T}, \max }: 0.34 \mathrm{~mm} \\
\mathrm{f}_{\mathrm{T}, \lim }: 17.00 \mathrm{~mm}\end{array}$ & $\begin{array}{l}\mathrm{f}_{\mathrm{A}, \max }: 0.13 \mathrm{~mm} \\
\mathrm{f}_{\mathrm{A}, \lim }: 8.50 \mathrm{~mm}\end{array}$ & PASSA \\
\hline $\mathrm{b}: \mathrm{P} 11-\mathrm{P} 8$ & $\begin{array}{l}f_{i, Q}: 0.02 \mathrm{~mm} \\
f_{\mathrm{i}, Q, \lim : 14.14 \mathrm{~mm}}\end{array}$ & $\begin{array}{l}\mathrm{f}_{\mathrm{T}, \max }: 0.12 \mathrm{~mm} \\
\mathrm{f}_{\mathrm{T}, \lim }: 19.80 \mathrm{~mm}\end{array}$ & $\begin{array}{l}\mathrm{f}_{\mathrm{A}, \max }: 0.03 \mathrm{~mm} \\
\mathrm{f}_{\mathrm{A}, \lim }: 9.07 \mathrm{~mm}\end{array}$ & PASSA \\
\hline c: P8 - P5 & $\begin{array}{l}\mathrm{f}_{\mathrm{i}, \mathrm{Q}}: 0.00 \mathrm{~mm} \\
\mathrm{f}_{\mathrm{i}, \mathrm{Q}, \lim }: 2.93 \mathrm{~mm}\end{array}$ & $\begin{array}{l}\mathrm{f}_{\mathrm{T}, \max }: 0.00 \mathrm{~mm} \\
\mathrm{f}_{\mathrm{T}, \lim }: 4.10 \mathrm{~mm}\end{array}$ & $\begin{array}{l}\mathrm{f}_{\mathrm{A}, \max }: 0.00 \mathrm{~mm} \\
\mathrm{f}_{\mathrm{A}, \lim }: 2.05 \mathrm{~mm}\end{array}$ & PASSA \\
\hline a: P5 - P2 & $\begin{array}{l}f_{\mathrm{i}, \mathrm{Q}}: 0.01 \mathrm{~mm} \\
\mathrm{f}_{\mathrm{i}, \mathrm{Q}, \lim }: 8.00 \mathrm{~mm}\end{array}$ & $\begin{array}{l}\mathrm{f}_{\mathrm{T}, \max }: 0.01 \mathrm{~mm} \\
\mathrm{f}_{\mathrm{T}, \lim }: 3.85 \mathrm{~mm}\end{array}$ & $\begin{array}{l}\mathrm{f}_{\mathrm{A}, \max }: 0.02 \mathrm{~mm} \\
\mathrm{f}_{\mathrm{A}, \lim }: 3.50 \mathrm{~mm}\end{array}$ & PASSA \\
\hline a: P16 - P12 & $\begin{array}{l}f_{i, Q}: 0.02 \mathrm{~mm} \\
f_{\mathrm{i}, Q, \lim : 12.14 \mathrm{~mm}}\end{array}$ & $\begin{array}{l}\mathrm{f}_{\mathrm{T}, \max }: 0.29 \mathrm{~mm} \\
\mathrm{f}_{\mathrm{T}, \lim }: 17.00 \mathrm{~mm}\end{array}$ & $\begin{array}{l}\mathrm{f}_{\mathrm{A}, \max }: 0.12 \mathrm{~mm} \\
\mathrm{f}_{\mathrm{A}, \lim }: 8.50 \mathrm{~mm}\end{array}$ & PASSA \\
\hline $\mathrm{b}: \mathrm{P} 12-\mathrm{P} 6$ & $\begin{array}{l}f_{\mathrm{i}, \mathrm{Q}}: 0.00 \mathrm{~mm} \\
\mathrm{f}_{\mathrm{i}, \mathrm{Q}, \text { lim }}: 10.43 \mathrm{~mm}\end{array}$ & $\begin{array}{l}\mathrm{f}_{\mathrm{T}, \max }: 0.02 \mathrm{~mm} \\
\mathrm{f}_{\mathrm{T}, \lim }: 4.81 \mathrm{~mm}\end{array}$ & $\begin{array}{l}\mathrm{f}_{\mathrm{A}, \max }: 0.01 \mathrm{~mm} \\
\mathrm{f}_{\mathrm{A}, \lim }: 7.30 \mathrm{~mm}\end{array}$ & PASSA \\
\hline c: P6 - P3 & $\begin{array}{l}f_{i, Q}: 0.00 \mathrm{~mm} \\
f_{i, Q}, \lim : 8.00 \mathrm{~mm}\end{array}$ & $\begin{array}{l}\mathrm{f}_{\mathrm{T}, \max }: 0.07 \mathrm{~mm} \\
\mathrm{f}_{\mathrm{T}, \lim }: 11.20 \mathrm{~mm}\end{array}$ & $\begin{array}{l}\mathrm{f}_{\mathrm{A}, \max }: 0.03 \mathrm{~mm} \\
\mathrm{f}_{\mathrm{A}, \lim }: 5.60 \mathrm{~mm}\end{array}$ & PASSA \\
\hline
\end{tabular}




\section{CONCLUSÃO}

Através deste projeto foi possível pôr em prática os conhecimentos adquiridos nas disciplinas de Estruturas de concreto armado (Concreto I e II) e arquitetura.

Podemos concluir que a tecnologia BIM tem facilitado muito a verificação de erros e a integração entre as disciplinas de modo a otimizar a obra, evitando prejuízos.

Outro ponto que cabe destacar é que embora o cálculo tenha sido todo elaborado através de um software é necessário ter um conhecimento técnico e normativo, isto fica evidente no pré-dimensionamento dos elementos estruturais uma vez que não foram usadas seções (tanto de pilar quanto de viga) de dimensionamentos absurdos, e também cabe ao profissional interpretar os erros que podem ocorrer no momento em que é feito o dimensionamento. 


\section{Anexo I - Catálogo da Engemolde}

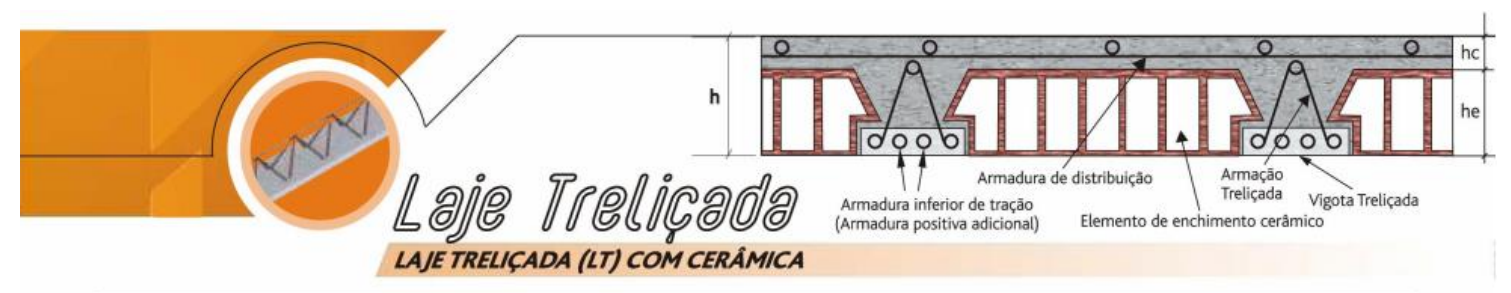

\begin{tabular}{|c|c|c|c|c|c|c|c|c|c|c|c|c|c|}
\hline \multirow{4}{*}{ TIPO } & \multicolumn{13}{|c|}{ Tabelas de Vãos Máximos para Lajes Treliçadas (LT) Unidirecional } \\
\hline & \multicolumn{7}{|c|}{$\begin{array}{l}\text { BI-APOIADA } \\
\text { Carga (Aça) Adicional Total (p) - } \mathrm{KN} / \mathrm{m}^{2} \\
\text { (carga acidental(q) }+* \\
\text { carga permanente adicional (g)(1) }\end{array}$} & \multicolumn{6}{|c|}{$\begin{array}{c}\text { APOIADA / ENGASTADA } \\
\text { Carga (Açab) Adicional Total (p) - KN/m² } \\
\text { (carga acidental( }(q)+ \\
\text { carga permanente adicional (g)(1) }\end{array}$} \\
\hline & \multicolumn{3}{|c|}{$\Delta$} & \multicolumn{4}{|c|}{$\Delta$} & & \multirow{2}{*}{3,5} & \multirow[b]{2}{*}{5,0} & \multirow[b]{2}{*}{7,5} \\
\hline & 1,0 & 2,0 & 2,5 & 3,5 & 5,0 & 7,5 & 10,0 & 1,0 & 2,0 & 2,5 & & & \\
\hline LT $10(7+3)$ & 4,30 & 4,10 & 4,01 & 3,67 & 3,18 & 2,68 & 2,41 & 4,92 & 4,67 & 4,56 & 3,95 & 3,41 & 2,85 \\
\hline LT $11(7+4)$ & 4,56 & 4,37 & 4,28 & 4,04 & 3,50 & 2,95 & 2,65 & 5,18 & 4,95 & 4,84 & 4,36 & 3,76 & 3,14 \\
\hline LT $11(8+3)$ & 4,78 & 4,57 & 4,47 & 4,19 & 3,64 & 3,06 & 2,75 & 5,40 & 5,15 & 5,04 & 4,52 & 3,90 & 3,26 \\
\hline LT $12(8+4)$ & 5,04 & 4,84 & 4,75 & 4,58 & 3,97 & 3,35 & 3,01 & 5,69 & 5,44 & 5,34 & 4,95 & 4,27 & 3,58 \\
\hline LT $16(12+4)$ & 6,02 & 6,01 & 6,00 & 5,85 & 5,30 & 4,47 & 4,01 & 6,76 & 6,52 & 6,41 & 6,21 & 5,75 & 4,82 \\
\hline LT $20(16+4)$ & 7,17 & 6,95 & 6,85 & 6,67 & 6,28 & 5,59 & 5,02 & 8,02 & 7,76 & 7,65 & 7,44 & 6,88 & 6,00 \\
\hline LT $25(20+5)$ & 8,45 & 8,24 & 8,14 & 7,95 & 7,70 & 6,66 & 6,00 & 9,40 & 9,15 & 9,04 & 8,83 & 8,54 & 7,27 \\
\hline \multicolumn{14}{|c|}{ Informaçōes técnicas das lajes treliçadas (LT) com EPS - UNIDIRECIONAL. } \\
\hline TIPO & & $\begin{array}{l}\text { Altura } \\
\text { total } \\
\text { da laje } \\
\text { h) }(\mathrm{cm})\end{array}$ & \multicolumn{3}{|c|}{$\begin{array}{l}\text { Altura do } \\
\text { elemento de } \\
\text { enchimento } \\
\text { (he) }(\mathrm{cm})\end{array}$} & \multicolumn{2}{|c|}{$\begin{array}{l}\text { Capa de } \\
\text { concreto } \\
\text { (C) }(\mathrm{cm})\end{array}$} & \multicolumn{3}{|c|}{$\begin{array}{c}\text { Ação } \\
\text { permanente } \\
\text { de peso próprio } \\
\left(\mathrm{KN} / \mathrm{m}^{2}\right)\end{array}$} & \multicolumn{3}{|c|}{$\begin{array}{l}\text { Concreto para } \\
\text { capeamento (C) } \\
\text { e nervuras (NL) } \\
\left(\mathrm{m}^{3} / \mathrm{m}^{2}\right)\end{array}$} \\
\hline LT $10(7+3)$ & & 10 & & \multicolumn{2}{|l|}{7} & & 3 & \multicolumn{3}{|c|}{1,53} & \multicolumn{3}{|c|}{0,040} \\
\hline LT $11(7+4)$ & & 11 & \multicolumn{3}{|c|}{7} & & 4 & \multicolumn{3}{|c|}{1,77} & \multicolumn{3}{|c|}{0,050} \\
\hline LT $11(8+3)$ & & 11 & & \multicolumn{2}{|l|}{8} & & 3 & \multicolumn{3}{|c|}{1,70} & \multicolumn{3}{|c|}{0,045} \\
\hline LT $12(8+4)$ & & 12 & & \multicolumn{2}{|l|}{8} & & 4 & \multicolumn{3}{|c|}{1,94} & \multicolumn{3}{|c|}{0,055} \\
\hline LT $15(12+3)$ & & 15 & & 12 & & & 3 & & 2,17 & & & 0,057 & \\
\hline LT $16(12+4)$ & & 16 & & 12 & & & 4 & & 2,42 & & & 0,067 & \\
\hline LT $20(16+4)$ & & 20 & & 16 & & & 4 & & 2,94 & & & 0,080 & \\
\hline LT $25(20+5)$ & & 25 & & 20 & & & 5 & & 3,57 & & & 0,102 & \\
\hline & & & & lormas & Técnic & cas (A & ABNT) & & & & & & \\
\hline NBR 6118 - Pro & to de e & estruturas & s de con & oncreto - & Procedi & Simento & & & & & & & \\
\hline NBR 9062 - Pro & to e ex & recuçāo $\mathrm{d}$ & le estrut & uturas de & e concre & eto pré- & -moldad & & & & & & \\
\hline NBR 6120 - Car & as para & o cálculc & de est & struturas & de edifi & ficações. & & & & & & & \\
\hline NBR 14859-1 - & aje Pré- & -Fabricad & la - Requ & quisitos - & - Parte & 1: Lajes & s unidirec & ecionais & & & & & \\
\hline NBR 14859-2 - & aje Pré- & Fabricad & la - Req & quisitos - & - Parte & 2: Lajes & s bidirecic & cionais; & & & & & \\
\hline NBR 14860-1 - & aje Pré- & Fabricad & la Pré-L & -Laje - Re & equisito & os - Part & te 1: Laje & jes unid & direcionai & & & & \\
\hline NBR $14860-2$ - & aje Pré- & Fabricad & la Pré-L & -Laje - Re & equisito & os - Part & te 2: Laje & jes bidir & irecionais & & & & \\
\hline NBR 14862 - A & naduras & streliçad & as eletro & trosoldad & & equisito & & & & & & & \\
\hline
\end{tabular}
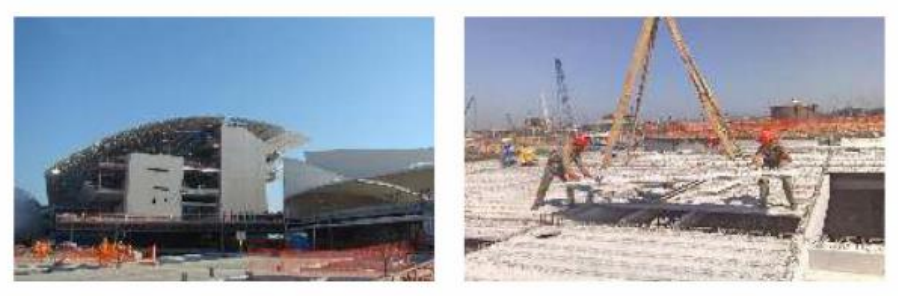

Consideraçōes

1 Previsto o valor de $0,50 \mathrm{KN} / \mathrm{m}^{2}$ de carga de revestimento como carga permanente adicional;

2 Para lajes bidirecionais, outras alturas e cargas (açöes), consulte o Departament Técnico da ENGEMOLDE:

3 LT - Laje treliçada unidirecional (NBR - 14859-1);

4 Concreto de capeamento estrutural classe C25 (Fck $\geq 25 \mathrm{MPa}$ );

$51,0 \mathrm{KN} / \mathrm{m}^{2}=100 \mathrm{kgf} / \mathrm{m}^{2}$

6 Vão máximo em metros;

7 Para LT $10(7+3)$ e LT $11(7+4)$, considerar um intereixo de $45 \mathrm{~cm}$, para demais lajes considerar intereixo de $38 \mathrm{~cm}$;

8 O cálculo, dimensionamento e demais definiç̧es såo elaborados pela ENGEMOLDE no projeto das lajes.

(Foto esquerda)

Lajes treliçadas

Novo Centro de Pesquisas da Petrobras - CENPES - R Consórcio Novo Cenpes

(OAS / Carioca / Construcap / Schahin / Construbase)

(foto direita)

Pré-Lajes

Prage Tanks

CSA - companhia Siderúrgica do Atlantico Consórcio OAS / Engevix / Steuler 


\section{REFERÊNCIAS BIBLIOGRÁFICAS}

ASSOCIAÇÃO BRASILEIRA DE NORMAS TÉCNICAS-ABNT. NBR 6118: Projeto de estruturas de concreto - Procedimento - elaboração. 2007. São Paulo: ABNT, 2014.

ASSOCIAÇÃO BRASILEIRA DE NORMAS TÉCNICAS-ABNT. NBR 6120: Cargas para o cálculo de estruturas de edificações. 1994. Rio de Janeiro: ABNT, 1994.

ASSOCIAÇÃO BRASILEIRA DE NORMAS TÉCNICAS-ABNT. NBR 9050:

Acessibilidade a edificações, mobiliário, espaços e equipamentos urbanos - Elaboração. 2015. Rio de Janeiro: ABNT, 2015.

ASSOCIAÇÃO BRASILEIRA DE NORMAS TÉCNICAS-ABNT. NBR 6122: Projeto e execução de fundações - elaboração. 2010. São Paulo: ABNT, 2010.

ENGEMOLDE. Catálogo de Laje Treliçada (LT) com cerâmica. Disponível em: <http://www.engemolde.com.br/uploads/produtos/laje_trelicada_ceramica.pdf $>$. Acesso em: 28 out. 2021.

SANTOS, N. V. Compatibilização de projetos estruturais de edificações via metodologia BIM - integração entre softwares de modelagem e dimensionamento de projeto. Projeto de Graduação. Universidade do Estado do Rio de Janeiro, UERJ, Rio de Janeiro, Brasil, 2019

ANDRADE, R. V. Projeto estrutural automatizado de uma edificação em concreto armado. Projeto de Graduação. Universidade Veiga de Almeida, UVA, Rio de Janeiro, 2017.

SOFTWARE CYPECAD - VERSÃO 2017.

SOFTWARE REVIT - VERSÃO 2021.

SOFTWARE AUTOCAD - VERSÃO 2019.

APRENDA O QUE É O IFC E QUAL A SUA IMPORTÂNCIA PARA O BIM.

Makebim, 2017. Disponível em: < https://www.makebim.com/2017/02/14/aprenda-oque-e-o-ifc-e-qual-a-sua-importancia-para-o-bim/> . Acesso em: 16 novembro 2021

O CONCEITO BIM (BUILDING INFORMATION MODEL). Saepro, [2015?].

Disponível em: <https://www.ufrgs.br/saepro/saepro-2/conheca-o-projeto/o-conceitobim-building-information-model/>. Acesso em: 16 novembro 2021

SOFTWARE DE BIM MULTIDISCIPLINAR PARA PROJETOS COORDENADOS E DE MAIOR QUALIDADE. Autodesk, [2015?]. Disponível em: <https://www.autodesk.com.br/products/revit/overview>. Acesso em: 16 novembro 2021 


\section{Pontificia U Universidade C $_{\text {atólica }}$ DO RIO DE JANEIRO}

CYPECAD SOFTWARE PARA CÁLCULO ESTRUTURAL. Autodesk, [2015?]. Disponível em: 〈https://www.autodesk.com.br/products/revit/overview〉. Acesso em: 16 novembro 2021 